\section{RESEARCH ONLINE}

University of Wollongong Thesis Collection
University of Wollongong Research Online

2013

\title{
The phonological neighbourhood effect on short- term memory for order
}

Larissa Clarkson

University of Wollongong

\section{Recommended Citation}

Clarkson, Larissa, The phonological neighbourhood effect on short-term memory for order, Doctor of Philosophy thesis, School of Psychology, University of Wollongong, 2013. http://ro.uow.edu.au/theses/3841 


\section{UNIVERSITY OF WOLLONGONG COPYRIGHT WARNING}

You may print or download ONE copy of this document for the purpose of your own research or study. The University does not authorise you to copy, communicate or otherwise make available electronically to any other person any copyright material contained on this site. You are reminded of the following:

Copyright owners are entitled to take legal action against persons who infringe their copyright. A reproduction of material that is protected by copyright may be a copyright infringement. A court may impose penalties and award damages in relation to offences and infringements relating to copyright material. Higher penalties may apply, and higher damages may be awarded, for offences and infringements involving the conversion of material into digital or electronic form. 


\section{The phonological neighbourhood effect on short-term memory for order}

A thesis submitted in fulfilment of the requirements for the award of the degree

Doctor of Philosophy

from

UNIVERSITY OF WOLLONGONG

By

Larissa Clarkson. B Psyc. (Hons)

School of Psychology 


\section{Thesis Certification}

\section{CERTIFICATION}

I, Larissa Clarkson, declare that this thesis, submitted in fulfilment of the requirements for the award of Doctor of Philosophy, in the School of Psychology, University of Wollongong, in wholly my own work unless otherwise referenced or acknowledged. The document has not been submitted for qualifications at any other academic institution.

Larissa Clarkson 


\begin{abstract}
Verbal short-term memory and long-term memory were once thought to be distinct processes, with short-term memory responsible for the maintenance and manipulation of verbal information in the immediate consciousness, and long-term memory responsible for storage of words and associated information over the lifespan.
\end{abstract} However, more recent research has suggested that the processes are intrinsically linked. The ability to learn novel items suggests that short-term memory is able to contribute to long-term memory, and recent findings also indicate that long-term memory has an influence on short-term memory. Typically, long-term memory is thought to assist the recall of items from short-term memory through a late stage redintegration process but does not influence the serial ordering of items in memory. More recently, one long-term memory variable, phonological neighbourhood density has been shown to influence memory for order in an immediate serial recall task with lists containing both large and small phonological neighbourhood words. This result has implications for the locus of the link between long-term memory and short-term memory, and is indicative of a link occurring earlier than assumed by most models of short-term memory. The aim of this thesis was to replicate evidence that phonological neighbourhood density is a variable that is sensitive to order processing and to identify the conditions under which this sensitivity appears. In a series of experiments, the effect of phonological neighbourhood density on memory for order was examined using tasks that varied in their requirement to recall order information or item information. Memory for order was better for words from large neighbourhoods than from small neighbourhoods, for pure lists but not for mixed lists in a task that reduces the requirement to remember item information, namely serial reconstruction. Further experiments using this paradigm revealed that this result was not due to differences in word lengths between large and small stimuli sets nor due to differences in reaction time to the items at output, and could not be attributed to different processes underlying immediate serial recall and serial reconstruction. The difference in memory for order between large- and small- phonological neighbourhood lists was found to disappear under conditions of articulatory suppression, suggesting that sub-vocal articulation acts as an intermediary between long-term memory and short-term memory. Finally, a second long-term memory variable, word frequency, was examined and was also shown to influence memory for 
order. This is indicative of a general long-term memory effect on short-term memory for order that has not been identified previously in the literature. These results are discussed in line with models of memory for order and are found to be most compatible with psycholinguistic models of memory that view order as a pattern of activation across a set of lexical units within a common system for memory and language. 


\section{Acknowledgements}

Firstly, I'd like to thank my supervisor, Steven Roodenrys for his patience, support and guidance. I'd also like to acknowledge my co-supervisor Leonie Miller for helping me through this process. To my family, thank you for your emotional and financial support, and thank you for resisting the temptation to ask me when I'll finally be finished. Thanks to my friends, fellow students and colleagues, with a special thanks to my hetero life mate Megan. 


\section{Table of Contents}

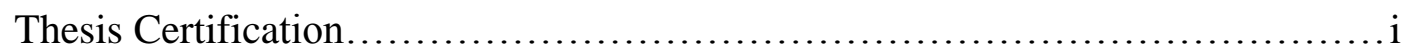

Abstract......................................................................

Acknowledgements........................................................

List of Figures.....................................................

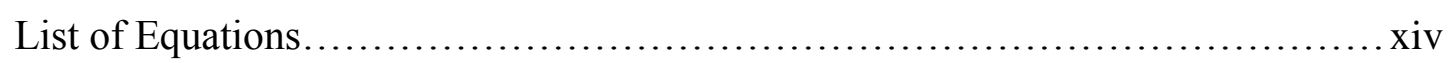

Chapter One $\quad$ Introduction................................. 1

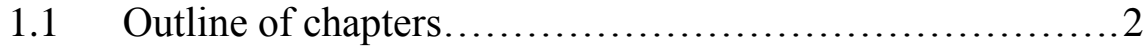

Chapter Two Memory for Verbal Information: Past Debates and Current Conceptions...........................4

2.1 Memory for Verbal Information: An Overview............... 4

2.1.1 Short-Term Memory and Long-Term Memory: Separate or

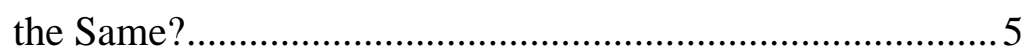

2.1.2 Verbal Memory and its Relationship to the Linguistic System........................................................ 8

2.2 A Closer Examination of the Verbal Short- and Long-Term

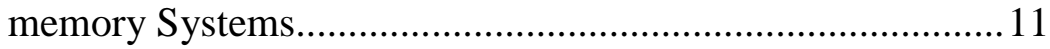

2.2.1 Long-Term Memory: The Lexicon........................................ 11

2.2.2 An Overview of Verbal Short-Term Memory....................... 12

2.2.2.1 Short-Term Memory: The Working Memory Model............ 13

2.2.3 Assessing Short-Term Memory: Immediate Serial Recall.... 14

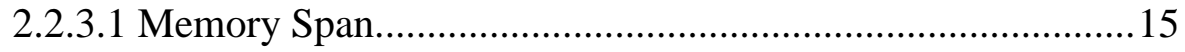

2.2.3.2 Performance on Supraspan Lists..........................................16

2.3 Assessing the Limits of Short-Term Memory ...................... 17

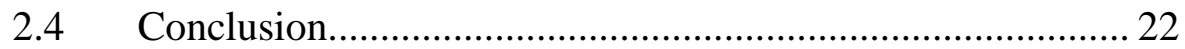

Chapter Three Inadequacies of Trace Decay plus Rehearsal..... 24

3.1 Introduction......................................... 24

$3.2 \quad$ The Lexicality Effect................................ 24 
3.3 The Word Frequency Effect........................... 25

3.4 The Language Familiarity Effect........................2 28

3.5 The Semantic Similarity Effect..........................29

3.6 Concreteness and Imageability Effects....................30

3.7 Connectivity with Long-Term Memory: The Redintegration Hypothesis.......................................... 31

3.7.1 A Dynamic Network Model of Redintegration.............. 34

3.7.2 Explaining the Lexico-Semantic Effects using Redintegration....................................... 35

3.8 The New Working Memory Model: Baddeley (2001).......... 36

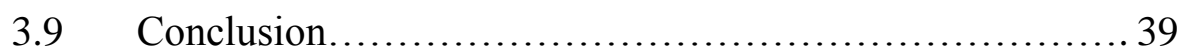

Chapter Four Effects of the Lexical Neighbourhood.............40

$4.1 \quad$ Introduction.......................................... 40

4.2 The Phonological Neighbourhood Effect.................... 41

4.2.1 Defining the Phonological Neighbourhood.................. 41

4.3 The Phonological Neighbourhood Effect on Speech Perception........................................... 43

4.3.1 The Neighbourhood Activation Model.......................45

4.3.2 All Neighbours are Equal in Lexical Space..................47

4.4 The Phonological Neighbourhood Effect on Speech Production............................................. 49

4.4.1 Two-Step Interactive-Activation Model of Lexical Access.. 50

4.5 Effects in the Visual Domain: Neighbourhood Effect on Perceiving Written Words.............................. 53

4.5.1 Models of Visual Word Perception...........................54

4.6 The Phonological Neighbourhood Effect in Short-Term Memory............................................ 55

4.7 The Neighbourhood Frequency Effect.................... 58

4.8 Explaining the Neighbourhood Effect within the Redintegration Model...................................................59

4.9 Phonotactic Frequency Effect............................6 62 
4.10 The Role of the Lexical Neighbourhood in New Word Learning.

4.11 Conclusion............................................ 64

Chapter Five Short-Term Memory for Order................... 66

5.1 The Problem of Serial Order.............................. 66

5.2 Two Things to Remember: Item and Order Memory........ 67

5.3 Inadequacy of the Immediate Serial Recall Task............ 71

5.3.1 Analysis of Order Errors.............................. 71

5.3.2 Conditional Probabilities: Immediate Serial Recall Task.....73

5.3.3 Serial Order Recognition............................... 74

5.3.4 Order Reconstruction................................... 74

5.3.4 Other Measures of Memory for Order..................... 75

5.4 Lexico-Semantic Effects on Memory for Order............. 76

5.4.1 The Lexicality Effect on Memory for Order................. 76

5.4.2 The Word Frequency Effect on Memory for Order.......... 77

5.4.3 The Language Familiarity Effect on Memory for Order..... 78

5.4.4 The Concreteness Effect on Memory for Order............ 78

5.5 Adapting the Redintegration Hypothesis: The Retrieval-Based Hypothesis........................................ 79

5.6 The Phonological Neighbourhood Effect on Short-Term Memory for Order. 80

5.7 Explaining the Phonological Neighbourhood Effect on Memory for Order: Inadequacies of the Retrieval-Based Hypothesis..81

5.8 Conclusion 82

\section{Chapter Six Models of Memory for Order} .84

6.1 An Overview of Models............................... 84

6.2 Chaining Models......................................... 86

6.3 Positional Models............................................ 89

6.4 Ordinal Models............................................ 91

6.5 Memory for Order as part of the Language System.......... 93

6.6 Conclusion.............................................. 96 
Chapter Seven Investigating the Phonological Neighbourhood Effect on Short-Term Memory for Order......... 97

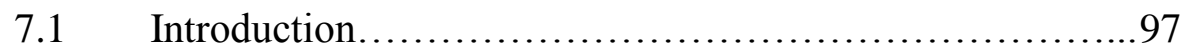

7.2 Experiment 1: Investigating the Neighbourhood Effect in Serial Recall using Mixed Lists................................. 98

7.2.1 Rationale............................................ 98

7.2.2 Hypotheses.............................................. 99

7.2.3 Method................................................. 99

7.2.3.1 Participants....................................... 99

7.2.3.2 Stimuli.......................................... 100

7.2.3.3 Procedure........................................ 101

7.2.4 Results............................................. 101

7.2.4.1 Order Errors..................................... 102

7.2.5 Discussion.......................................... 103

7.3 Experiment 2: Phonological Neighbourhood Effects on Serial Recognition: Mixed Lists............................... 105

7.3.1 Rationale............................................. 105

7.3.2 Hypotheses........................................ 106

7.3.3 Method................................................. 106

7.3.3.1 Participants....................................106

7.3.3.2 Stimuli......................................... 107

7.3.3.3 Procedure........................................ 107

7.3.4 Results............................................... 108

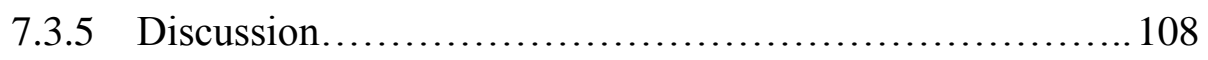

7.4 Experiment 3: Phonological Neighbourhood Density Effects in Serial Recall and Serial Recognition using Pure Lists..... 109

7.4.1 Rationale.............................................. 109

7.4.2 Hypotheses.......................................... 110

7.4.3 Method.............................................. 110

7.4.3.1 Participants....................................110

7.4.3.2 Stimuli........................................... 111

7.4.3.3 Procedure.............................................. 111

7.4.4 Results............................................ 112 
7.4.4.1 Serial Recall.................................... 111

7.4.4.2 Error Analysis.................................. 114

7.4.4.3 Serial Recognition..............................115

7.4.5 Discussion............................................... 115

7.5 General Discussion.................................... 119

\section{Chapter Eight The Phonological Neighbourhood Effect on}

Short-Term Memory for Order: Serial

Reconstruction................................... 120

$8.1 \quad$ Introduction............................................ 120

8.2 Experiment 4: The Phonological Neighbourhood Effect in a Serial Reconstruction Task: Mixed Lists................... 121

8.2.1 Rationale............................................. 121

8.2.2 Hypotheses....................................... 122

8.2 .3 Method............................................. 122

8.2.3.1 Participants................................... 122

8.2.3.2 Stimuli....................................... 122

8.2.3.3 Procedure..................................... 122

8.2.4 Results............................................... 123

8.2.4.1 Error Analysis................................... 124

8.2 .5 Discussion......................................... 125

8.3 Experiment 5: The Phonological Neighbourhood Effect in a Serial Reconstruction Task: Pure Lists.................. 126

8.3.1 Rationale........................................... 126

8.3.2 Hypotheses............................................. 126

8.3.3 Method.................................................. 126

8.3.3.1 Participants..................................... 126

8.3.3.2 Stimuli........................................ 127

8.3.3.3 Procedure.................................... 127

8.3.4 Results.............................................. 127

8.3.5 Discussion........................................ 128

8.3.6 Combining Experiment 4 and Experiment 5............. 131

8.3.7 Discussion............................................. 133 
8.4 Experiment 6: Phonological Neighbourhood Effects in a Serial Reconstruction Task using Mixed and Pure Lists............ 134

8.4.1 Rationale................................................ 134

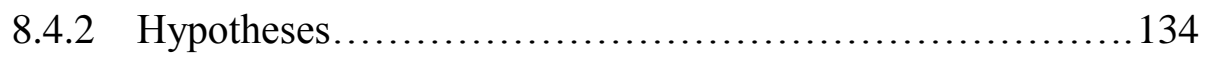

8.4.3 Method................................................. 135

8.4.3.1 Participants.................................. 135

8.4.3.1 Stimuli.......................................... 135

8.4.3.2 Procedure....................................... 135

8.4 .4 Results................................................ 136

8.4.5 Discussion............................................... 138

8.5 General Discussion.................................. 139

Chapter Nine The Phonological Neighbourhood Effect on Memory for Order: Replicating the Effect.......141

$9.1 \quad$ Introduction............................................. 141

9.2 Experiment 7: Eliminating the Effect of Word Length....... 142

9.2.1 Rationale........................................... 142

9.2.2 Hypotheses............................................ 143

9.2.3 Method.................................................. 144

9.2.3.1 Participants....................................144

9.2.3.2 Stimuli......................................... 144

9.2.3.3 Procedure...................................... 144

9.2 .4 Results................................................ 145

9.2.5 Discussion............................................... 148

9.3 Experiment 8: Removing Speech Production using Articulatory Suppression.......................................... 149

9.3.1 Rationale........................................... 149

9.3.2 Hypotheses...................................... 150

9.3.3 Method.............................................. 151

9.3.3.1 Participants.......................................151

9.3.3.2 Stimuli.......................................... 151

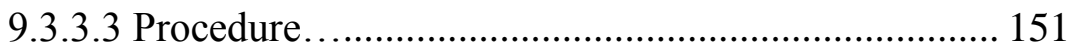

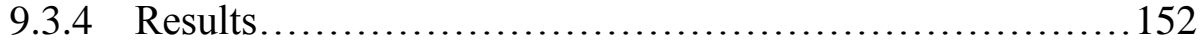

9.3.5 Discussion........................................... 153 
9.4 Experiment 9: Removing the Influence of Blocking: Serial Reconstruction of Order with Articulatory Suppression....................................... 153

9.4.1 Rationale............................................. 153

9.4.2 Hypotheses.......................................... 154

9.4.3 Method............................................. 154

9.4.3.1 Participants................................... 154

9.4.3.2 Stimuli......................................... 155

9.4.3.3 Procedure......................................... 155

9.4 .4 Results.............................................. 155

9.4.5 Discussion.............................................. 156

9.5 Experiment 10: Immediate Serial Recall and Serial Reconstruction of Order: Post-Cued......................159

9.5.1 Rationale................................................ 159

9.5.2 Hypotheses........................................... 160

9.5.3 Method................................................... 160

9.5.3.1 Participants......................................160

9.5.3.2 Stimuli.......................................... 161

9.5.3.3 Procedure....................................... 161

9.5.4 Results............................................ 162

9.5.5 Discussion............................................ 163

9.6 Conclusion............................................ 165

\section{Chapter Ten The Word Frequency Effect on Memory for}

Order....................................................... 166

10.1 Introduction........................................ 166

10.2 Experiment 11: Investigating Other Lexical Variables: The Effects of Word Frequency and Phonological Neighbourhood size on Memory for Order.....................................167

10.2.1 Rationale............................................. 167

10.2.2 Hypotheses....................................... 168

10.2.3 Method............................................. 168

10.2.3.1 Participants................................... 168 
10.2.3.2 Stimuli................................................. 169

10.2.3.3 Procedure............................................. 169

10.2.4 Results.................................................... 170

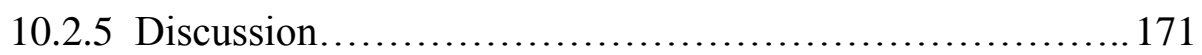

Chapter Eleven A Recapitulation of Current Research.......... 176

11.1 An Overview of the Research Project.......................176

11.2 The Phonological Neighbourhood Effect on Memory for

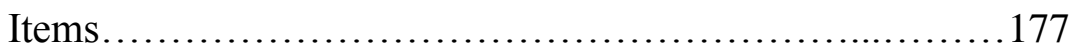

11.2.1 Mixed Lists........................................ 177

11.2.2 Pure Lists.......................................... 178

11.3 The Phonological Neighbourhood Effect on Memory for

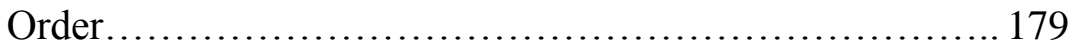

11.3.1 Mixed Lists........................................... 179

11.3.2 Pure Lists.......................................... 180

11.3.3 The Effect of Articulatory Suppression.............. 183

11.4 The Word Frequency Effect on Short-Term Memory for

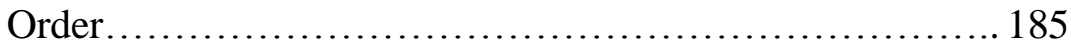

11.5 Conclusion............................................. 187

\section{Chapter Twelve Implications of the Current Findings and Future} Research Directions.................................188

12.1 Introduction............................................ 188

12.2 Implications for the Retrieval-Based Hypothesis............. 188

12.3 Implications for Models of Memory for Order................190

12.3.1 Conclusions.................................................196

12.4 Suggestions for Future Research........................... 197

12.5 Conclusion................................................... 199

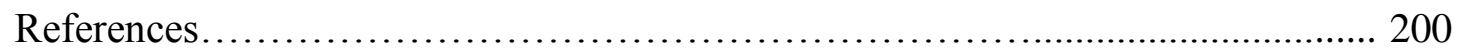

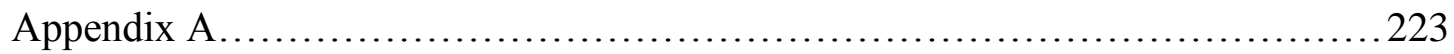

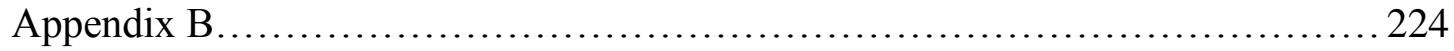




\section{List of Figures}

Figure 2.1 The DISLEX model of the human lexicon (Miikkulainen, 1997)....... 11

Figure 2.2 The working memory model proposed by Baddeley and Hitch (1974).. 14

Figure 3.1 Multinomial processing tree model (Schweickert, 1993)............. 32

Figure 3.2 Revised working memory model (Baddeley, 2001)................ 37

Figure 3.3 Functional model of the phonological loop (Baddeley, 2005)........ 38

Figure 4.1 Part of the phonological lexicon examined in Vitevitch (2008)........ 42

Figure 4.2 Flow chart for the neighbourhood activation model

(Luce \& Pisoni, 1998).

Figure 4.3 The lexical network for the interactive two-step model of naming (Dell, Schwartz et al., 1997)

Figure 4.4 Martin et al.'s (1999) levels of representation in speech perception and production. 60

Figure 6.1 Simple and compound chaining models (Henson, 1996)..... 87

Figure 6.2 Representation of serial order in an ordinal model (Henson, 1996)... 92

Figure 7.1 Mean serial recall performance across serial position as a function of phonological neighbourhood size in Experiment 1 102

Figure 7.2 Mean serial recall performance across serial position as a function of phonological neighbourhood size in Experiment 3.

Figure 8.1 Mean number of items correctly ordered by serial position for alternating lists of large and small phonological neighbourhood words in Experiment 4

Figure 8.2 Reconstruction performance across serial position by phonological neighbourhood size in Experiment 5 . 
Figure 8.3 Combining Experiment 4 and Experiment 5: Serial reconstruction performance by neighbourhood size and list condition across serial position

Figure 8.4 Serial order reconstruction performance across serial position as a function of neighbourhood size and list type in Experiment 6.

Figure 9.1 Mean proportions correct across serial position for large and small phonological neighbourhood words in Experiment 7.

Figure 9.2 Serial reconstruction performance by block order (large neighbourhood words first or small neighbourhood words first) across serial position in Experiment 7

Figure 9.3 Reaction time in milliseconds across serial position for large and small neighbourhood words in Experiment 7.

Figure 9.4 Mean proportion of items placed in the correct position for large and small neighbourhood sets in Experiment 8

Figure 9.5 Serial reconstruction performance by block order (large neighbourhood words first or small neighbourhood words first) across serial position in Experiment 9

Figure 9.6 Serial reconstruction and serial recall performance for large and small neighbourhood lists in Experiment 10

Figure 10.1 Mean proportion correct for the four stimuli conditions in Experiment 11

Figure 12.1 Theoretical model of the relationship between ISR, non-word repetition and word learning (Gupta, 2003) 


\section{List of Equations}

Equation 2.1 Memory span equation

Equation 3.1 Revised memory span equation to account for a long-term memory influence (Hulme et al., 1991). 31

Equation 3.2 Probability of correct item recall from Schweickert's (1993) model of redintegration 33

Equation 4.1 Luce \& Pisoni’s (1998) Neighbourhood probability rule. 46 


\section{Chapter One}

\section{Introduction}

Human memory is multifaceted, capable of storing not only a large quantity but also a wide variety of information. The structure of cognitive processes and resources that give rise to this ability has been at the crux of past and present debate (e.g., Baddeley \& Hitch, 1974; Henson, Norris, Page \& Baddeley, 1996; Nairne, 2002). Arguably, one of the most essential types of human memory is memory for verbal information, as it is this type of memory that enables us to interact meaningfully with others. Theorists espouse a variety of possible structures of memory for language, with the leading instantiation involving a division of labour between systems storing permanent representations of words, known as long-term memory, and those managing verbal information in our immediate consciousness (Baddeley, 2002).

Despite this division, there is a wealth of information suggesting that the two systems are intrinsically linked, with evidence supporting a facilitative long-term memory influence on the short-term maintenance of verbal information (Hulme, Maughan, \& Brown, 1991; Hulme, Roodenrys, Schweickert, Brown, Martin, \& Stuart, 1997). The amount of information that can be maintained in the short-term is typically assessed using a serial recall task, where participants are required to repeat a sequence of just-presented words. One piece of evidence that suggests long-term memory influences short-term memory is the phonological neighbourhood effect on serial recall (Roodenrys, Hulme, Lethbridge, Hinton, \& Nimmo, 2002). A phonological neighbourhood is a cohort of words that share a similar sound structure with a target word. These neighbouring words can assist recall of a target word from short-term memory. This manifests as a recall advantage for lists of words with many phonological neighbours over words with few phonological neighbours, even when the neighbours do not appear within a test list. The phonological neighbourhood effect in short-term memory tasks is the subject of this thesis.

Previous research had suggested that this long-term memory influence occurs quite late in the short-term memory process and its purpose is to reinstate item information that has been lost due to decay. However, Roodenrys et al. (2002) found that a word's phonological neighbourhood also affected the order of output. This 
finding was noteworthy as order information is assumed to be recalled before the reconstruction process begins. This finding is also incongruous with previous research showing long-term memory does not influence order recall (Saint-Aubin \& Poirier, 1999b).

The present work aims to investigate this novel effect of phonological neighbourhood density on short-term memory for order. The overarching aim is to identify the loci of the exchange between long-term memory and short-term memory, and to explicate the mechanisms driving this process.

\subsection{Outline of Chapters}

The following provides a brief description of the thesis content by chapter.

Chapter 2 provides an outline of the past and current debates in memory for verbal information. This chapter presents an overview of several possible instantiations of verbal memory including a unitary system view, a dual system view, and a confluence between verbal memory and language systems. Following this, evidence for the capacity limits of short-term memory are presented within a tracedecay plus rehearsal model of short-term memory.

In Chapter 3, evidence for the inadequacy of the trace-decay plus rehearsal model is presented. An alternative approach that allows for a long-term memory influence is introduced, as well as a discussion of a formal model of the long-term memory influence, known as the redintegration hypothesis.

Chapter 4 reviews evidence that words in the mental lexicon other than those present in short-term memory may influence short-term recall; the phonological neighbourhood effect. An in-depth review of the literature surrounding the phonological neighbourhood effect on speech perception, speech production and short-term memory is provided. Models of perception and production are outlined and the Neighbourhood Activation Model is described.

In Chapter 5, the concept of memory for order is discussed in more detail. Methods of separating item and order information in short-term memory are described. This chapter outlines the current research in memory for order with a focus on the finding of a phonological neighbourhood effect on memory for order. 
Chapter 6 outlines models of memory for order. The chapter begins with an historical review of modelling and outlines some key benchmarks of serial ordered behaviour that models must account for. Several models are presented and how well these models can account for key findings, including the phonological neighbourhood effect, is discussed.

The next four chapters outline the empirical component of the current work. Chapter 7 presents the experimental data from the first series of experiments. These data examine the phonological neighbourhood effect on memory for items and memory for order. The second series of experiments is presented in Chapter 8. This series investigates the phonological neighbourhood effect in a more "process pure" test of memory for order, namely serial reconstruction. Serial reconstruction reduces the requirement to recall item information by providing items at recall. A significant neighbourhood effect was found, indicating a long-term memory influence on shortterm memory for order. A number of possible alternative explanations of the effect were investigated in Chapter 9. Findings from this series of experiments revealed that the effect of neighbourhood density is not due to differences in word length or an effect of response latency, and is not due to differences between the recall task and the reconstruction task. Further experiments indicated that the neighbourhood effect disappears when the ability to rehearse is disrupted, suggesting the locus of the effect is in speech production. Chapter $\mathbf{1 0}$ examines the extent to which the findings apply to other long-term memory variables by looking at the effect of word frequency on short-term memory for order.

A recapitulation of the findings is presented in Chapter 11. The results are discussed in light of the hypotheses and the aims of the research. While in Chapter 12, the findings are discussed in relation to models of memory for order. In this final chapter, limitations of the current research series are outlined, as well as suggestions for future research. 


\section{Chapter Two}

\section{Memory for Verbal Information: Past Debates and Current Conceptions}

\subsection{Memory for Verbal Information: An Overview}

Memory is the ability to retain and recall events from the past, and knowledge about the world. The application of memory ranges from remembering a name to knowing how to drive a car, making it vital for day-to-day human functioning. Verbal memory is the ability to encode, maintain and retrieve linguistic information, including phonological, lexical and semantic representations. Phonological representation refers to the individual sounds that make up a word, with a phoneme being the smallest distinct unit of speech that differentiates one word from another (e.g., /d / /ô/ /g/ is distinguished from /d / /ô/t/ by the last phoneme). Lexical representation refers to the whole word form (e.g., dog). Finally, semantic representation refers to the meaning of words (e.g., four-legged companion animal). Verbal memory has implications for language processing and production, and is regarded by some as the seat for human thought and consciousness (Hulme, Newton, Cowan, Stuart, \& Brown, 1999).

Verbal memory has been the subject of experimental research for around 200 years. Ebbinghaus inaugurated the experimental tradition of examining verbal memory, commencing his work in the late $19^{\text {th }}$ century, which culminated with his monograph Memory (Cofer, 1976). Ebbinghaus was primarily concerned with the capacity limits of retention, and it was not until James (1890) that discussions began about the morphology of memory. James (1890) made a distinction between primary memory and secondary memory, which roughly corresponded to the modern notions of short-term memory and long-term memory respectively. An object in primary memory, according to James, is one that comes from the "reawarded portion of the present space of time" while an item in secondary memory "is one which has been absent from consciousness altogether, and now revives anew" (James, 1890, p. 609). 
While this initial concept of verbal memory was of a dual system, subsequent theorists have espoused various systems of verbal memory including a unitary memory system and a memory system that exists as an extension of linguistic processes.

\subsubsection{Short-Term Memory and Long-Term Memory: Separate or the Same?}

There is a schism between theorists espousing long-term memory and shortterm memory as distinct cognitive structures, and those supporting a unitary system view where long- and short-term memory systems operate along a continuum (Baddeley, 2002). Historically, most interpretations of verbal memory ascribed to the former view and involved a division of labour between systems dealing with items in immediate attention, and those managing the permanent representations of known words (Broadbent, 1957). Permanent representations are contained within a long-term memory system, which is often referred to as the mental lexicon, while short-term memory has become synonymous with a working memory system, with a history of diverse manifestations. Short-term memory is a more labile system, which allows words to be processed, maintained over a short period of time, and manipulated if necessary. Although viewed as separate, these systems are linked with short-term memory viewed as instrumental for learning by passing information to long-term memory for more permanent storage (Gathercole \& Baddeley, 1989).

A number of theorists have argued strongly against the notion of separate short- and long-term memory systems stating that traditional short-term memory findings could easily be explained by "factors known to operate in long-term memory" (Melton, 1963, p. 8). Proponents of a unitary memory system conceive of verbal short-term memory as activated long-term memory. Temporal distinctiveness theories for instance, argue for a common underlying memorial mechanism that operates on different time scales (Glenberg \& Swanson, 1986). Cowan (1988, 1995, 1999) described an embedded process model to explain how short-term recall could be captured within a unitary system. According to his model, working memory is temporarily activated long-term memory that is under the focus of attentional control. This temporary activation enables the items to be accessed only when attention is focussed on them. The attentional control process is assumed to be of a limited 
capacity, thus explaining capacity limits associated with short-term memory. However, this limited capacity attentional process is not unique to the embedded process model, nor is it unique to a unitary system view. Acheson and MacDonald (2009b) draw similarities between the attentional control process in Cowan's model and aspects of dual systems such as Broadbent's (1957) supervisory attentional system and Baddeley's (1986) central executive.

There is a plethora of evidence both for and against the dual system view. While a comprehensive account of these arguments is beyond the scope of the present work, a number of key arguments will be discussed.

Early experimental evidence for a differentiation between long- and short-term memory systems came from the Hebb repetition effect (Hebb, 1961). Hebb found that memory for a sequence improved relative to an overall task improvement in performance if that sequence were presented frequently. This was thought to reflect the repeated string gaining representation in long-term or secondary memory, pointing to a division between the systems.

Additional arguments for separable systems came from evidence of different rates of forgetting between long-term memory and short-term memory. Evidence of this sort came from performance on the Brown-Peterson task (Brown, 1958; Peterson \& Peterson, 1959), where participants were asked to recall items below their expected span (usually around three items per list) after a retention period that varied between 3 and 30 seconds. During the retention period, participants performed a distracter task different enough from the primary task as to not interfere with it, but difficult enough to prevent rehearsal of the list items. By varying the length of the distracter task, researchers were able to ascertain how long items stayed in short-term memory. Typically, after a relatively short delay (e.g., 20 seconds) performance on the BrownPeterson task is almost at floor (Neath, 2006). This demonstration of the limits of short-term memory stands in stark contrast to the relative permanency of long-term memory, pointing to a dual store. According to dual store theorists, information is lost during the Brown-Peterson task due to decay operating in short-term memory. However, proponents of the unitary system cite additional evidence that suggests that loss of information during the Brown-Peterson task occurs due to proactive interference rather than decay (see Surprenant \& Neath, 2009, for a review). Proactive interference occurs when information presented previously intrudes in the current 
task, preventing the recall of the newer information. Traditionally, the concept of decay was favoured by dual-system theorists as an explanation for loss of information in the short-term, while proactive interference is believed to be a long-term memory effect, absent from short-term memory tasks. Surprenant and Neath (2009) argued that proactive interference can be induced in any short-term memory task so long as the experimenter ensures that the intruding stimulus is properly processed. By calling into question the concept of decay as an explanation of short-term loss, and showing that proactive interference could occur in tasks traditionally thought to measure shortterm memory, unitary system theorists suggested that a separate short-term store was unnecessary (Surprenant \& Neath, 2009).

Nairne and Neath (2001) further criticised the suggestion that variations in performance on short- and long-term memory tasks was evidence for a dual-store system, based on what they saw as a fundamental flaw in the logic of tests of immediate serial recall. They criticised the view that immediate serial recall, typically used as a test of short-term memory, measures anything other than a general processing capacity. They investigated long-term memory capacity by using a delayed recall task, where recall was deferred until five minutes after presentation. They found that participants could retain around five items, which is similar to the capacity limit of short-term memory. Their point was not to suggest that the capacity limit of longterm memory was indeed five items, but to draw attention to what they propose is a fault in the logic of immediate serial recall as a test of short-term memory. They suggested that rather than being an item capacity limit per se, the limitation in memory may simply be a characteristic of information load, that is, there may be only so much information we can correctly order regardless of the time scales involved.

Perhaps the most compelling evidence for separate systems arises from investigations of impaired populations, with double dissociations between amnesic patients with deficient long-term memory and intact short-term memory systems, and patients with deficient short-term memory processes alongside intact, or relatively intact, linguistic processes and long-term memory systems. Tulving (2002) noted that such deficiencies were difficult to explain "unless exists the potentiality for such a division [between long-term memory and short-term memory] in the healthy brain" (p. 12). However, Surprenant and Neath (2009) note several alternate explanations for amnesic performance that do not subsume separable memory processes, such as an 
inability for amnesics to bind the memory of an event to its context, or an increase in the amount of interference from other stimuli for patients with amnesia over normal populations. Further evidence from neuropsychological studies in normal populations challenge this explanation. Collette et al. (2001) examined Positron Emission Tomography (PET) scanning techniques on normal adults. They found separate areas of activation for tasks thought to rely solely on short-term memory, and tasks believed to involve verbal long-term memory, although they did report some interplay between the systems when examining the short-term retention of words. Collette et al. (2001) interpreted their results in line with separate long-term memory and short-term memory systems; although they noted that their results were also consistent with the view of verbal memory as part of the language system.

Two possible instantiations of verbal memory have been discussed, the view of long-term memory and short-term memory as distinct systems, and as a unitary system. These instantiations are by no means exhaustive with a number of theorists espousing a third memory system. Cowan (1984) for instance, suggested a third store, termed the long auditory store, which was purported to retain auditory information for around 20 seconds. This was based on evidence that traces could support the recall of item information (but not order information) beyond that expected from short-term memory (Mueller \& Krawitz, 2009). A different additional system was proposed by Ericsson and Kintsch (1995) to account for instances of expert performance. This was termed long-term working memory and was created to explain the speed of retrieval of well-practiced information.

\subsubsection{Verbal Memory and its Relationship to the Linguistic System}

In addition to being viewed as part of long-term memory, theoretical interpretations of memory for language have also suggested a confluence with linguistic research. Given verbal short-term memory maintains language-based information, it is logical to assume that it would interact with phonological and semantic representations (Majerus, 2009). Ellis (1980) found commonalities between transposition errors in short-term memory and spoonerisms in speech. Based on his findings, Ellis suggested that both types of error arise at the same point in the information processing system, supporting the interdependence of systems dedicated 
to memory and those dedicated to language. Models of memory that lie within the linguistic sphere are called psycholinguistic models.

Much of the evidence for psycholinguistic models relies on data from impaired populations. N. Martin, Saffran, and Dell (1996) reported an individual with deep dysphasia who showed impairments typically associated with a deficit in phonological short-term memory. Deep dysphasia is a speech disorder characterised by word repetition problems and semantic errors in word repetition (Ablinger, Abel, \& Huber, 2008). N. Martin et al. (1996) demonstrated that the patient's impairments could be modelled using a computational model of single word production simply by varying the period of time between input and output as well as the decay rate. This challenged the idea of separable working memory and linguistic buffers and instead suggested that "auditory working memory performance depends on storage capacities intrinsic to the language processing system" (N. Martin et al., 1996, p. 83). Of course this is not conclusive evidence for a unitary mechanism, but an argument for such a mechanism can be made based on parsimony.

In another study, N. Martin and Saffran (1997) examined the short-term memory performance of aphasic patients who primarily had either a phonological impairment or a semantic impairment. The task varied with regards to the reliance on semantic and phonological information. The pattern of results suggested that representations were stored within a common language processing mechanism with participants able to support their recall using the unimpaired abilities. For instance, patients who had a phonological deficit with relatively intact semantic capabilities were more likely to show effects of semantic variables on repetition span, demonstrating an increased reliance on this type of information.

Further evidence supporting psycholinguistic models comes from a study of an anomic patient, MS (R. Martin et al., 1999). Anomia is a syndrome characterised by impairments in naming. Martin and her colleagues demonstrated that the patient's semantic and lexical processes were intact and that the deficiency was specific to the activation of phonological representations. The patient showed comparable performance on naming tasks and serial recall indicating the same mechanism underlies both processes. In contrast, he was not impaired on measures of speech perception and comprehension and performed well on memory tasks that did not require spoken output. This pattern of performance was used as evidence for 
immediate serial recall utilising processing in the speech output pathway, indicating that short-term memory and speech production have the same locus.

The interdependence of mnestic and linguistic systems has also been reported by theorists espousing a dual system account of memory and language. Gathercole and Baddeley (1989) showed that a group of children with a specific language impairment had both poor digit span and poor non-word repetition, suggesting a common underlying mechanism. This underlying mechanism, the researchers proposed, was the phonological loop component of Baddeley and Hitch's (1974) working memory model. The component was thought to rely on articulatory motor processes for rehearsal, whether articulation was covert or overt. Although this interpretation still resides within a dual system account, Gathercole and Baddeley also accepted that results could be accounted for by a common mechanism for memory and language.

Several prominent theories espousing a unitary system view have emerged in the past two decades. These are only briefly outlined here, with a more comprehensive account given in Chapter 6. Gupta and MacWhinney (1997) proposed an integrated linguistic system, responsible for both vocabulary acquisition and shortterm memory. They presented a computational model and identified possible neural substrates. R. Martin et al. (1999) presented an interactive model of language and short-term memory where phonological, semantic and lexical representations of words involved in language perception and production were closely associated with phonological and lexico-semantic buffers. Activation in these buffers is maintained though feedback from the long-term memory representations. Acheson and MacDonald (2009b) provided a more recent account, where memory and language are stored in the same multileveled system and activation is maintained through recurrent connectivity between nodes and layers.

While the debate over whether long- and short-term memory are separate or the same, or perhaps part of the linguistic system prevails, much of the previous experimental work in this field has accepted the two-system view. For this reason, the elements of the dual-system will be discussed in more detail. 


\subsubsection{Long-Term Memory: The Mental Lexicon}

The mental lexicon is a storage system for the long-term representations of words and associated information, such as semantic, phonological, and grammatical information. Due to the differences in input and output modalities and differences in the way items can be represented in long-term memory it is intuitive to suggest that the lexical system is highly modular (Miikkulainen, 1997). Miikkulainen (1997) created a Distributed model of the Lexicon (DISLEX) where information was arranged in topographical maps. Although several models of the mental lexicon exist most of these are nested within other systems. For instance, Levelt (1989) outlined a model of the mental lexicon, but this resided within the linguistic system rather than being a standalone model. For this reason, one standalone model of the mental lexicon, the DISLEX model will be discussed in detail. Models of the mental lexicon that reside within the psycholinguistic model are described in detail later in this thesis.

The DISLEX model, shown in Figure 2.1, has two layers. The first layer represents the lexical representation of words for both their orthographic, or written, and phonological, or speech-based, forms with both input and output maps. The second layer contains the semantic map.

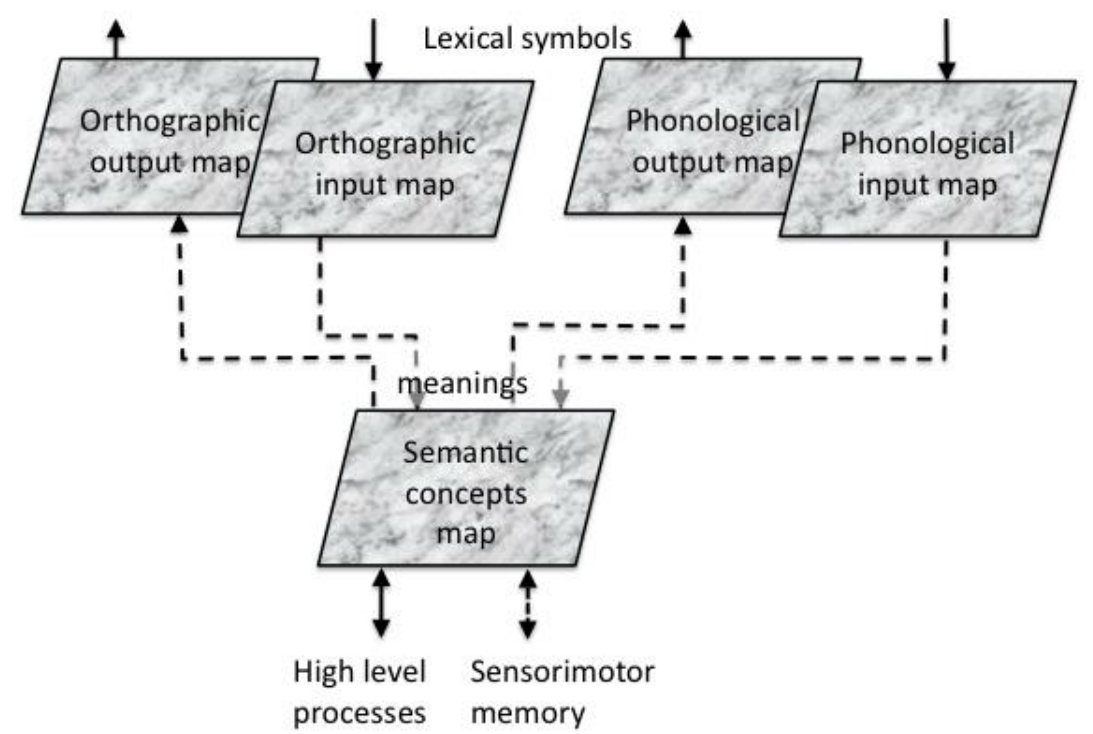

Figure 2.1 The DISLEX model of the human lexicon as represented in Miikkulainen (1997). The solid lines represent the propagation of distributed representation while the dashed lines indicate associative pathways. 
Lexical representations are stored with distributed representation in vectors with grey scale values between 0 and 1 . These lexical vectors have contact with the sensory modalities and with the semantic concept map. Like lexical information, word meaning is also stored within the semantic concept map as a distributed representation. As well as being connected to lexical representations, the semantic map is connected to higher level processing, which includes language processing and episodic memory, and to sensorimotor memory, which includes, among other things, a visual image of the semantic concept.

The two layers of representation are arranged in feature maps with each node representing a particular word or concept. Words with similar phonological or orthographic form are located in close proximity on those maps, and words similar in meaning are located in close proximity on the semantic concept map. A word's representation in the lexical symbol system is connected to the corresponding representation in the semantic concept map by bidirectional connections with weights that can be adjusted using Hebbian learning.

\subsubsection{An Overview of Verbal Short-Term Memory}

Verbal short-term memory refers to the extremely capacity limited information processing ability, containing all linguistic information that is in immediate consciousness (Hulme et al., 1999). The system is responsible for the short-term retention of speech-based information, such as remembering a phone number long enough to dial or remembering a set of directions, and plays a role in higher cognitive functioning. Specifically, short-term memory is regarded as a key determinant for language processing, production and learning (Gupta \& McWhinney, 1997), and is considered to be paramount for higher order reasoning and thinking (Baddeley, 1997) with mounting support for each of these claims. The following is a description of the historical development of the concept of short-term memory.

Models of short-term memory have evolved from capacities for the passive storage of speech-based information to a mental workspace where items can be cognitively manipulated as well as being stored. Atkinson and Shiffrin (1968) first coined the term "working memory", building on the information-processing model created by Broadbent (1958). They combined a short-term memory component and an 
attentional resource in a single store giving a model with three distinct stores. Information enters the system via sensory stores where it is useful for around one to three seconds before being transferred to a short-term store. Although Atkinson and Shiffrin (1968) labelled this system working memory, it became more widely recognised as the modal model. Atkinson and Shiffrin proposed that information was able to be updated from short-term memory to long-term memory if the items were rehearsed appropriately, but their account was shown incapable of accounting for learning effects. For instance, as short-term memory is instrumental in learning, the modal model would predict that disabling this system through dual task loading would disrupt learning. Instead, Baddeley and Hitch (1974) showed that participants could still learn quite effectively when their short-term memory was incapacitated by loading the system with a digit span task. The failure to model learning effects, amongst other problems, led to the replacement of the model with Baddeley and Hitch's (1974) working memory model as the prominent theoretical account.

Rather than the unitary short-term store proposed by Akinson and Shiffrin (1968), Baddeley and Hitch's (1974) model incorporated a visuo-spatial store, a verbal short-term memory system and a governing central executive. While Baddeley and Hitch's model makes no attempt to describe the mechanisms underlying encoding, maintaining and retrieving information, it acted as a framework on which much subsequent theoretical and experimental development in short-term memory took place. In addition, the Baddeley and Hitch (1974) account was the basis for much of the interpretation of a number of lexico-semantic effects that sired the current investigation. For these reasons, a more complete account of this model is presented below.

\subsubsection{Short-Term Memory: The Working Memory Model}

Baddeley and Hitch's (1974) multi-component working memory model describes a system responsible for managing information in the short term. It is called working memory because it is a system where information is actively manipulated rather than being passively stored (Baddeley, 2001). This system was originally comprised of three components, a governing central executive and two subsidiary systems, the phonological loop, which is responsible for managing speech based 
stimuli, and the visuospatial sketchpad, which manages the maintenance and manipulation of visual and spatial information. This is depicted in Figure 2.2.

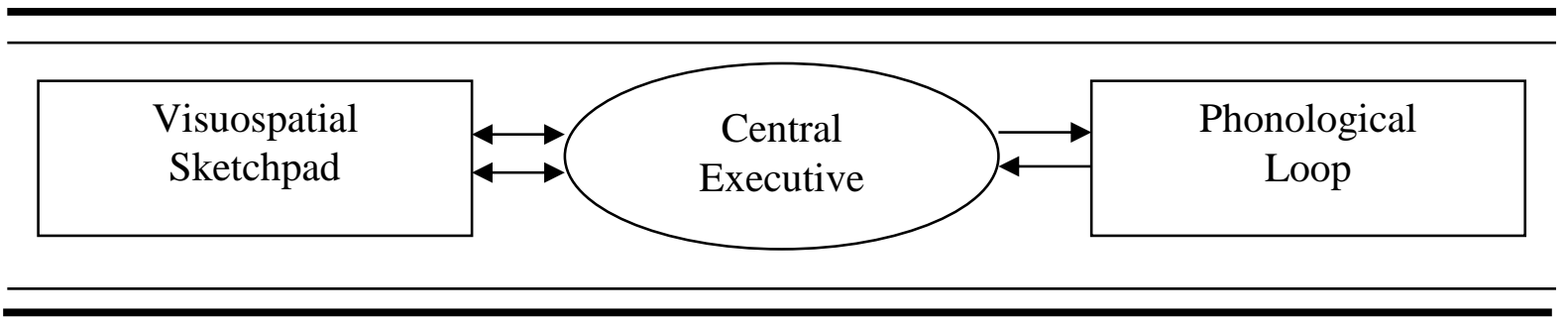

Figure 2.2. The working memory model proposed by Baddeley and Hitch (1974)

The working memory model has three key assumptions; $(a)$ buffers are used to maintain information over a short period of time, $(b)$ representations in the phonological store are phonological in nature, and $(c)$ loss from stores occur via passive decay. The working memory model was more recently updated by Baddeley (2001) to include additional systems. This revision will be discussed in Section 3.8 after a discussion of the evidenced that spawned the current model and the data that prompted the revised model.

The phonological loop is assumed to be central to language learning (Gathercole \& Baddeley, 1989), and provides a structural base to the influential trace decay plus rehearsal interpretation of the capacity limits of short-term memory (Hulme et al., 1999). According to the trace-decay plus rehearsal theory, speech-based information enters the phonological loop where it is subject to decay. Active rehearsal, either vocal or subvocal, circumvents decay. The assumptions around the phonological loop and trace-decay plus rehearsal theory are based on several observations of short-term memory performance primarily garnered from the immediate serial recall task.

\subsubsection{Assessing Short-Term Memory: Immediate Serial Recall}

Short-term memory capacity is traditionally assessed using an immediate serial recall task (ISR) where participants are asked to repeat a list of items immediately after they have been presented. Items may be numbers (as in a digit span task), words, non-words, or letters. Items can be presented visually or aurally and recall can be verbal or written. In ISR, items must be recalled in the order they were presented, which differs from free recall where participants can recall items in any 
order. Typically, an item is only scored as correct if it is the correct item in the correct serial position.

ISR can be used to assess capacity limits of short-term memory by ascertaining an individual's memory span, or can be used with supraspan lists, which are lists just above normal capacity limits. Supraspan lists are used to provide a more complex picture of how short-term memory operates when overloaded, and can provide a wealth of information based on what errors are made in each trial (Conrad, 1964).

\subsubsection{Memory Span}

Memory span is indicative of how many items a person can hold in their shortterm memory. The capacity of short-term memory is determined by the longest list an individual can recall without error, following a single presentation (Hulme et al., 1991), although this definition is not necessarily accepted by all researchers (i.e., Dosher \& Ma, 1998).

Memory span is presumed to be a function of the length of time a trace is useful before it begins to decay, and the rate at which items can be rehearsed.

This is given by the equation:

$$
s_{i}=r_{i} \tau \quad \text { [equation 2.1] }
$$

Where $s$ refers to memory span for items $i$, which is a linear function of the pronunciation rate, $r$, and the duration of the trace $\tau$. The duration of the trace is presumed to be a constant whereas the pronunciation rate may vary (Neath \& Nairne, 1995).

Memory span is a useful tool to examine the effect of different lexical variables on short-term memory, is used in neuropsychological research to gauge the effect different neurological conditions have on memory (see Milner, 1968) and is included in intelligence testing (e.g., Wechsler digit span scales; Wechsler, 1958). 


\subsubsection{Performance on Supraspan Lists}

Perhaps a more effective tool to examine short-term memory is the application of supraspan lists. Here participants perform ISR on lists above what they would be expected to be able to accurately recall. Trace-decay plus rehearsal theory proposes that errors in recall occur once the time needed to articulate the sequence length is greater than the decay time of the store. Estes (1972) observed, "when retention is imperfect, the confusion errors that occur are highly systematic" (p. 161). That is, by forcing imperfect retention, additional information may be garnered from an examination of recall errors, as these reflect the underlying processes. Data from supraspan lists can be assessed using a number of methods including a simple correctin-position analysis, or using a free recall criterion where participants are given a score for each correct item regardless of whether it was recalled in the correct position or not. As supraspan lists result in errors for most lists, error frequency and types can be examined to provide additional information about the complexities of short-term memory. Errors are broadly classified by whether they are indicative of an item error or an error in the positional information.

Item errors can be classified as omissions, where an item is not recalled in any position, or as intrusions. Intrusions can further be classified as extra-experimental intrusions, where words are replaced by ones that are not in the experimental item set and bear no significant similarity to an item presented in the list; intra-set intrusions, where words within the item set but not in the current list are recalled; phonological approximation errors, where intruding items share at least $50 \%$ of phonemes with an item in the present list; and semantic neighbourhood errors, where an item that is related in meaning to a list item is recalled instead of the list item, although this list is not exhaustive (Allen \& Hulme, 2006).

Errors in positional information have been often overlooked in analyses of ISR. Errors of this sort can be classified by the direction and distance of their movement, or simply by their frequency. Order errors are most commonly reported with regards to how frequently they occur within a list rather than by their type, and are often assessed in proportion to the number of items recalled correctly. A detailed examination of order error types is given in Chapter 5. 


\subsection{Assessing the Limits of Short-Term Memory}

Research on ISR has led to the identification of a number of important benchmarks that have been used to determine the capacity limits of the short-term memory system. These benchmarks were used as evidence for the nature of Baddeley and Hitch's (1974) working memory model, and must be accounted for in any new model of short-term memory. Key findings include the effects of list length, word length and the phonological and semantic similarity effects.

In addition, an analysis of these variables under conditions of articulatory suppression has been paramount in understanding short-term memory. Articulatory suppression involves repeating an irrelevant word or phrase, such as "the", while performing a short-term memory task. Suppression is thought to disrupt the use of speech processes and impede the participant's ability to rehearse items. A participant can be required to articulate the irrelevant word either during presentation, maintenance or recall, or any combination of these. Articulatory suppression has been shown to have a general detrimental effect on recall, although the magnitude of the effect differs for different types of material and for different presentation modalities (Baddeley, 2001).

\section{The List Length Effect}

The number of items presented to a participant for recall at any one time has an effect on the amount of words that will be recalled. Miller (1956) found that participants could typically remember seven plus or minus two items. This was used as the basis for capacity limits in Atkinson and Shiffrins (1968) modal model, and was also thought to reflect the limited capacity of the phonological loop.

Beaman (2006) examined the list length effect in a serial recall task and found that the pattern of results mimicked that found in free recall. That is, there were more items recalled in the correct serial position in longer lists than in shorter lists, although the proportion of items recalled was greater in the shorter lists than in the longer lists. 


\section{The Word Length Effect}

Performance in an ISR task was shown to be a direct function of the length of the words being recalled, with lists of short words recalled better than lists containing long words (Baddeley, Thomson, \& Buchanan, 1975). The effect is robust for lists presented both visually and aurally, and extends to non-words (Hulme et al., 1991). The word length effect is taken as evidence for a capacity limited rehearsal mechanism in short-term memory that relies on overt or covert articulation by the articulatory motor programs (Camos, Lagner, \& Barrouillet, 2009).

Further, the word length effect is abolished under conditions of articulatory suppression if the items are presented visually, but not if they are presented verbally unless suppression is required to continue throughout recall (Baddeley et al., 1975). The effects of articulatory suppression on visual but not verbal information were explained by the assumption that the store requires information to be coded phonemically. Visual information must be articulated for it to be coded phonemically, a process that cannot occur if participants are engaging in concurrent articulation. Auditory information has obligatory access to the phonological store and so is not similarly affected by articulatory suppression. Page and Norris (1998) suggest that the abolition of the effect on orally presented items when articulatory suppression continued through recall may be because Baddeley and his colleagues allowed participants to abbreviate the longer words at output, effectively equating the output response times.

The word length effect presented a challenge to common interpretations for Miller's (1956) magic number seven plus or minus two, by suggesting that the number of items that could be recalled was dependent on the length of those items. Baddeley and his colleagues (1975) tested whether it was the length of the words per $s e$, or whether spoken duration of the words determined the word length effect. They examined recall performance of two syllable words that were constant in regards to the number of syllables and phonemes but differed in their spoken duration. Lists containing words with longer spoken durations, such as Friday were recalled worse than lists of two-syllable words with shorter spoken durations such as wicket. Baddeley et al. (1975) suggested that the useful life-time of a trace was not determined by the number of items within short-term memory, but by the rate at 
which these items could be articulated. According to this approach, the word length effect occurs because shorter words are faster to articulate and so more of them can be rehearsed, which offsets decay. The articulation process is the responsibility of the phonological or articulatory loop in the working memory model.

Schweickert and Boruff (1986) identified the decay time of the store by examining the spoken duration and the frequency of correct recall for a number of different types of items including digits, consonant-vowel-consonant (CVC) words and nouns. They found that memory span correlated with a spoken duration of, on average, 1.88 seconds, but varied slightly between types of stimuli. This indicates that participants are able to retain as much information as they can rehearse in just under 2 seconds, an effect that has been replicated (Hulme et al., 1991, but see Mueller \& Krawitz, 2009) and is consistent across languages (i.e., see Ellis \& Hennelly, 1980).

The time-based approach to the word length effect is not without criticism. Cowan et al. (1992) questioned whether the word length effect arose during rehearsal of the items as had been suggested. They presented mixed lists of long and short words to participants, where either the first half of the list contained long words followed by short words or vice versa. Furthermore, the recall order was reversed on half of the trials, with participants unaware of the required recall order until the presentation was complete. If the word length effect is simply due to the absolute speech rate of the items, then performance should be equivalent across lists. However, Cowan and his colleagues found that performance was worse for lists starting with the longer words if recall was forward, and worse for lists ending in longer words if recall was backward. This was taken as evidence for the influence of output interference. Baddeley, Chincotta, Stafford and Turk (2002) examined this account using tasks that varied in their output requirements and, on the basis of results, suggested that the word length effect is due to a combination of output interference and rehearsal mechanisms.

While the word length effect is regarded as one of the best pieces of evidence for the trace-decay plus rehearsal view, this interpretation of the finding has still be the focus of debate (Jalbert, Neath, Bireta, \& Surprenant, 2011). Neath and Nairne (1995) proposed an alternative explanation for the word length effect, suggesting that longer words are more fragile than shorter words because they have more features that can potentially be lost. Therefore, longer words are more prone to forgetting than 
shorter words. Baddeley (2002) noted that this explanation fails to account for the finding that the word length effect disappears under conditions of articulatory suppression. Further, in a study by Caplan, Rochon, and Waters (1992), the word length effect was reversed when words were matched for the number of syllables and phonemes but differed with regards to articulatory duration, which was determined by using words with tense and lax vowels. Tense vowels have a longer spoken duration than lax vowels. Caplan et al (1992) did replicate Baddeley et al's (1975) finding but only when they did not control for the number of phonemes. They suggested that the word length effect is due to the complexity of the phonological structure rather than articulation rate. This has been challenged by Baddeley and Andrade (1994), who pointed out that Caplan et al. (1992) only had minimal differences between the spoken duration of items. Service (1998) found similar levels of recall for words that differed in spoken duration by not in phonological complexity. This supports the notion that the word length effect is actually a complexity effect rather than due to differences in articulatory duration. Finally, Jalbert et al. (2011) suggested that the word length effect could be better explained by differences in the lexical properties of long and short words, rather than by the length of the words. This explanation is further explored in Chapter 4.

\section{The Phonological Similarity Effect}

Conrad (1964) first demonstrated effects of phonological similarity during an attempt to discriminate errors of perception from errors occurring due to decay in verbal short-term memory. Conrad used two tasks to differentiate between errors, a visual immediate recall task and a perceptual task with no memory requirement. Any association between the errors in the two tasks would be taken as evidence for acoustic confusions occurring separate to those induced by errors in perception. Letters were presented in lists of six and were either phonologically similar or dissimilar to one another. He found that when a recall error was made, the erroneous letter was likely to be phonologically similar to the target letter. In a second experiment, Conrad presented the letters to participants in noise and had them immediately write down the letter. Again, phonologically similar letters more often replaced a target letter. Interestingly, the errors in recall were more similar to the 
acoustic confusions that presented in the perceptual identification task than to the original letter. Conrad noted that this pointed to participants using articulatory rehearsal processes rather than storing items in a visual code.

Ellis (1980, Experiment 1), obtained a similar result in a study where participants performed a serial recall task on five item lists. Movement errors were analysed as a function of the number of phonemic features shared between the consonant on the target item and the consonant on the recalled item. There was a strong relationship between featural similarity and the probability of movement errors, which showed that item similarity has a detrimental effect on order memory.

Healy (1974) also found that items with a similar sound (e.g., the letters $B, V$, $T, C$ ) are easier to remember than items that are dissimilar in sound (e.g., the letters $F$, $W, M, R)$, when simply looking at the total number of items recalled. However, when looking at the number of order errors, Healy found a detrimental effect of phonological similarity. It was suggested that participants can use a token (in this case rhyme) to aid recall (Bjork \& Healy, 1974). This token acts as a category cue, which can assist recall for the words but this advantage does not extend to memory for order.

The nature of phonological confusions was used as evidence for the phonological nature of the store component of the working memory model (Baddeley, 2002). It was assumed that the effect arose due to an inability to discriminate between acoustically similar traces that had become partially decayed in the store. This is in contrast to the finding that similarity of meaning has little effect or no effect in ISR (Baddeley, 1966b). The reverse of these effects is typically observed in long-term memory tasks with no effect of phonological similarity but a strong effect of semantic similarity (Baddeley, 1966a). These findings have been used to propose that the phonological store relies principally on phonological encoding, while the long-term memory system primarily relies on semantic codes (Baddeley \& Levy, 1971).

In addition, the phonological similarity effect is removed under conditions of articulatory suppression for visually presented items but not for auditorially presented items (Baddeley, Lewis, \& Vallar, 1984). This is taken as evidence that access to the store is automatic for spoken information, which enters the store directly from an aural source, while written items require articulation prior to accessing the store.

There is debate regarding whether the effect of phonological similarity represents an order-based error or whether the results could more accurately be 
classed as extra-list intrusions (Henson et al., 1996). This approach will be discussed in more depth in Chapter 5.

Lastly, it has been suggested that the phonological similarity effect is an artefact of the word length effect, with similar words perhaps slower to pronounce than dissimilar words. Schweickert, Guentert, and Hersberger (1990) examined the phonological similarity effect and pronunciation times for lists of similar and dissimilar words and found that under recall conditions, phonologically distinct items are pronounced at the same rate as phonologically similar items, which is contrary to expectations if the phonological similarity effect is a manifestation of the word length effect. Schweickert et al. (1990) attributed the effect of phonological similarity to the phonological store, with representation in the store longer for dissimilar words, whereas the word length effect was attributed to the articulatory rehearsal system.

\subsection{Conclusion}

Amongst the wealth of findings that inform the nature of verbal short-term memory, the benchmark findings of the word length effect, the list length effect, the phonological similarity effect and effects of articulatory suppression have been put forth as evidence for a trace-decay plus rehearsal theory of verbal short-term memory. The list length effect indicates that the system is capacity limited, able to retain around seven items. The word length effect suggests that the number of items able to be recalled depends on the length of those items, although whether this is articulatory length or phonological complexity remains subject to debate. The phonological similarity effect informs us that verbal short-term memory acts on speech-based information primarily. The phonological nature of the system is further evidenced by the removal of the phonological similarity effect under conditions of articulatory suppression for visually presented material but not for orally presented material. This list of benchmark findings is by no means exhaustive and there is a wealth of other findings that further inform the nature of verbal short-term memory. For instance, individual differences in working memory span can be attributed to differences in speech rate (Schweickert \& Boruff, 1986; Standing, Bond, Smith, \& Isely, 1980).

Although Baddeley and Hitch's (1974) working memory model has been outlined as the chief morphology of short-term memory, the term short-term memory 
Chapter Two: Memory for Verbal Information: Past Debates and Current Conceptions

as applied in this thesis refers to any model of verbal short-term memory that adheres to the following properties: (1) there are fundamentally different processes responsible for remembering items over the short-term and remembering items over the long-term; (2) loss of information from the short-term store occurs via decay; and (3) rehearsal can counteract the effects of decay. 


\section{Inadequacies of Trace Decay plus Rehearsal}

\subsection{Introduction}

Trace decay plus rehearsal has been a successful explanation of capacity limits of short-term memory, leading to its popularity as an account of list length and word length effects. Evidence suggests that factors beyond these physical properties of items such as the lexical and semantic properties of words can also influence the probability that an item will be recalled. For instance, words are more likely to be recalled than non-words, words that are frequently encountered in language can be recalled more easily than those that are infrequent, and words that are concrete, or easy to image, are easier to recall than abstract words. These lexico-semantic effects are difficult to explain within a simple trace decay plus rehearsal account as they necessitate a link with long-term memory.

\subsection{The Lexicality Effect}

The adequacy of trace decay plus rehearsal as an explanation of capacity limits of short-term memory was brought into question by an investigation of ISR of lists of words and non-words. Trace decay plus rehearsal theory predicts no difference between the recall rate of items that have long-term memory, or lexical, representation (such as words) compared to items that do not have long-term memory representation (non-words), assuming they are matched on rehearsal or pronunciation rate. Hulme et al. (1991) assessed memory for words and non-words using an ascending memory span paradigm, while also gathering speech rate data to account for the role of articulatory processes. Hulme and his colleagues found that lists of words were recalled significantly better than lists of non-words, and that speech rate was equivalent for words and non-words. The speech rate finding suggests that the contribution of rehearsal to performance was the same for words and non-words, 
indicating that a simple trace decay plus rehearsal explanation was untenable. The advantage afforded to words, termed the lexicality effect, was instead attributed to words having a long-term memory representation.

Hulme, Roodenrys, Brown, and Mercer (1995) sought support for this explanation by examining whether learning non-words would reduce the lexicality effect. If the lexicality effect is due to interactions with long-term memory, then forging long-term lexical representation for non-words through learning should attenuate the lexicality effect. Hulme et al. confirmed an advantage for words over non-word stimuli in an ISR task. The non-words then underwent a process of familiarisation to create a long-term representation. Participants were examined on the items again following a 24-hour delay. Familiarisation increased the recall of nonwords relative to the recall of words. The magnitude of the effect could not be explained as an increase in the rate at which non-words could be articulated following training, and was not due to semantic processes supporting the recall of the non-words as no definitions were given during training.

Saint-Aubin and Poirier (2000) examined ISR performance on words and nonwords under quiet conditions and under conditions of articulatory suppression. Based on the premise that articulatory suppression increases the degradation of items, the researchers hypothesised that the condition would adversely affect non-words more than it would affect words. This is because non-words do not have lexical representation that could be used to replace the information lost due to articulatory suppression, while words are represented in long-term memory. They found a significant effect of lexicality under quiet conditions with superior recall for words than for non-words. Overall performance was depleted in the articulatory suppression condition as expected, but non-words were affected by suppression more than words. This was taken as evidence that the long-term representation of words was assisting their recall. The proposed mechanisms of this influence will be discussed in Section 3.7.

\subsection{The Word Frequency Effect}

Word frequency is an index of how often words are encountered in language (Baayen, Piepenbrock, \& Rijan, 1993). Word frequency is measured by how often a 
word appears, usually per million words, in a corpus of written text or spoken language (Stuart \& Hulme, 2000). A number of databases have been created to allow for easy access to word frequency counts (e.g. CELEX - Baayen et al., 1993; Kucera \& Francis, 1967). Word frequency had been shown to exert an influence on many cognitive tasks including lexical decision (Scarborough, Cortese, \& Scarborough, 1977), perceptual identification (Manelis, 1977), naming (Forster \& Chambers, 1973; Goldinger, Azuma, Abramson, \& Jain, 1997), and delayed repetition (Allen \& Hulme, 2006). Results of these tasks indicate that frequent words are produced faster (Jescheniak \& Levelt, 1994) and with fewer errors (Dell, 1988). In addition, high frequency words are recognised faster in noise than their low frequency counterparts (Allen \& Hulme, 2006).

Trace-decay plus rehearsal theory predicts that word frequency would not influence short-term memory beyond any advantage afforded by superior perception and production of high frequency words. Watkins (1977) examined this prediction using ISR, finding that performance was superior for high frequency words than for low frequency words, although this was later attributed to differences in the pronunciation rate of such items (Wright, 1979). Tehan and Humphreys (1988) examined whether the frequency effect could be explained within the trace-decay plus rehearsal system in this way, by examining ISR performance on high and low frequency words under quiet conditions and under conditions of articulatory suppression. If differences between the word sets were due to differences in articulation rate, then the effect should disappear when concurrent articulation is required. In contrast, the researchers found a stable word frequency effect under conditions of articulatory suppression, suggesting that the effect is due to processes occurring outside of rehearsal.

Hulme et al. (1997) tested this further using a serial recall task, while also examining possible differences in pronunciation rate using a speech rate measure. They found a facilitative effect of word frequency, with superior recall for lists of high frequency words than for those containing infrequent words. Further, while it was found that common words could be articulated faster than rare words, the effect on recall was still highly significant when speech rate was used as a covariate. Hulme and his colleagues interpreted the result as indicative of a link with long-term memory. 
It is evident from the research outlined thus far that the frequency of a word influences the likelihood that it will be recalled from short-term memory, and that this is due to links with long-term memory. It is unclear whether the frequency effect is an item level effect or an effect of list composition. Specifically, it is uncertain whether high frequency words are better recalled because of intrinsic properties of their longterm representations or whether recall is better because high frequency words have better long-term associative links (Saint-Aubin \& LeBlanc, 2005). Deese (1960) proposed that high frequency words would have a higher frequency of co-occurrence with other words than would low frequency words. Deese suggested that it was the frequency of co-occurrence rather than the frequency of the words that was behind the frequency effect.

Stuart and Hulme (2000) examined this possibility by manipulating the interitem associations between high- and low-frequency words. Half of the participants were familiarised with pairs of words to create inter-item associative links and the other half were not. Common words were familiarised with other common words and rare words were familiarised with other rare words. Both groups completed a serial recall task with lists of high and low frequency words as well as lists that alternated high and low frequency words. While familiarisation did not increase serial recall performance overall, performance was increased for pure lists of low frequency words in this condition. Performance on pure lists of low frequency items after familiarisation was equivalent to performance on pure lists of high frequency items, which had remained unchanged. This improvement in recall could not be explained in terms of an increase in the frequency of the items themselves as there was no effect of familiarisation on mixed lists of high and low frequency words. This indicates that familiarisation removed the advantage for high frequency words, suggesting that the word frequency effect is due to the greater likelihood that high frequency words cooccur with one another in language.

The word frequency effect has also been argued to be due to a confound with age of acquisition. Age of acquisition is the approximate age a word is first encountered. Often words that are encountered earlier in childhood are words that are also more frequent in language and so the effects of both age of acquisition and word frequency are often entangled (Newman \& German, 2002). Age of acquisition has been shown to influence a number of different cognitive tasks including reading speed (Barry, Hirsh, Johnston, \& Williams, 2001) and lexical decision speed (Morrison \& 
Ellis, 1995). Further, age of acquisition has been shown to mediate word frequency effects on naming and perceptual identification (Newman \& German, 2002). Roodenrys, Hulme, Alban, Ellis, and Brown (1994) investigated whether age of acquisition could be responsible for the word frequency effect in serial recall by manipulating word frequency and age of acquisition in two memory span tasks. They found a significant word frequency effect in memory span, but failed to find an effect of age of acquisition. They concluded that the word frequency effect is independent of age of acquisition.

\subsection{Language Familiarity Effect}

Further evidence for a long-term memory influence on recall comes from effects in bilinguals. Thorn, Gathercole, and Frankish (2002) examined lexicality in French/English bilinguals with either French or English as their first language. English and French vocabulary tests revealed participants had above average first language vocabulary and average second language vocabulary. Participants were tested on words and non-words for both their first and second language. A separate set of non-words were created for each language to ensure the non-words complied with the different phonotactic rules of English and French. Thorn and her colleagues found a significant first language advantage in ISR for both words and non-words, as well as a significant lexicality effect for both bilingual subgroups, with words recalled better than non-words. No interaction between language and lexicality was found, indicating that the effect of lexicality was consistent for both first and second languages. It was possible that the language familiarity effect could be due to output delay. That is, faster articulation in the first language may reduce the opportunity for decay to occur during output. Articulation in the second language tends to be slower giving more opportunity for time-based degradation. Thorn et al. (2002) tested this possibility by using a probed recall task. Probed recall is assumed to reduce output interference as participants are only required to recall a single item in a cued position. As in recall, bilinguals showed superior performance for their primary language, and showed a lexicality effect that was consistent for both the first and second languages. As the language familiarity effect persisted in probed recall, it was unlikely that the effect observed in serial recall was due to output interference. Instead the effect was 
attributed to support from long-term memory. Thorn et al. (2002) suggested that the language familiarity effect might be an artefact of word frequency, as words in the primary language are learnt first and are used more frequently than those in the secondary language. This does not explain the superior performance for non-words in the first language compared with non-words in the second language, although Thorn and her colleagues suggested that this may be due to a phonotactic frequency effect. This effect will be explored further in Chapter 4.

\subsection{The Semantic Similarity Effect}

Baddeley (1966a) investigated similarity effects in ISR using lists that were either phonologically similar (e.g., man, mad, map) or similar in meaning (e.g., big, huge, tall). Baddeley found a robust detrimental effect of phonological similarity across three experiments. In one experiment, Baddeley also looked at the influence of semantic similarity. The results were less compelling, with a small but significant effect of similarity of meaning with better ISR for similar sequences than for dissimilar sequences. In their 1970 study, Ecob and Baddeley showed effects of both semantic and phonological similarity in ISR. After a 20 second delay, the effect of phonological similarity disappeared while the effect of semantic similarity persevered. This was taken to indicate that the effects of phonological similarity occur in short-term memory while effects of semantic similarity occur due to influences from long-term memory.

While the effect of semantic similarity is small, it is robust, being replicated in a number of experiments (see, for instance, Saint-Aubin \& Poirier, 1999a). Despite this, the semantic similarity effect has often been disregarded because it has less of an effect on ISR performance than phonological similarity. This is because semantic similarity reflects a long-term memory influence on recall, while the phonological similarity effect appears to reflect the operations of the short-term memory system itself. As ISR is a task of short-term memory, it would be expected that factors that influence short-term memory such as phonological similarity would be more robust in this task than those that influence long-term memory such as semantic similarity. 


\subsection{Concreteness and Imageability Effects}

Word concreteness and imageability are similar concepts, and ratings of these variables are highly correlated. Words that easily generate a visual image of the word (e.g., tree) are termed high imageability words, whereas low imageability words are quite difficult to generate a visual image for (e.g., dignity). Concreteness is defined as the extent to which a word denotes a material object rather than an abstract quality or state of action (Walker \& Hulme, 1999). These two concepts are highly correlated because it is typically easier to generate an image of a material object than it is for an abstract quality. There are of course instances where a word may not be concrete but may be easy to imagine, for instance, the word 'happy'.

Trace-decay theory predicts that concreteness and imageability differences would not affect the recall of words in ISR. To test this, Walker and Hulme (1999) examined ISR performance on lists of concrete words and lists of abstract words of different word lengths. As expected, more short words were recalled than long words. Contrary to trace-decay plus rehearsal theory, more concrete words were recalled than abstract words. This was consistent with both verbal and written recall, and with both forward and backward recall. Backwards recall is purported to disrupt the use of a phonological code in serial recall, based on the finding that the word frequency effect is eliminated during backwards recall (Hulme et al., 1997). The finding that the concreteness effect is not eliminated in backwards recall was taken as evidence that concreteness affects the availability of a semantic code that underpins working memory. In addition to challenging trace-decay plus rehearsal, the finding is also evidence against the view that the short-term memory system relies solely on a phonological code, and instead suggests that semantic codes may also assist recall.

Allen and Hulme (2006) examined concreteness in speech perception, speech production and short-term memory tasks in an attempt to identify the system or systems that are affected by this variable. Concreteness effects were identified on the serial recall task, with an advantage for concrete words over abstract words. Performance in the word identification in noise task was not affected by concreteness indicating that this variable does not influence speech perception. There was a significant effect on the accuracy of definition naming, which suggests that recall may 
have been affected by how successful the semantic representation could assist the speech output representations at recall.

These results may be explained using Paivio's (1986) dual coding approach. Here, words that are easily imagined are stored in memory using both the phonological code and a visual code. Words that are difficult to imagine are stored using only the phonological code, as a corresponding visual code is not readily available. At recall, participants are able to draw on both phonological and semantic features to retrieve the lexical item. These results are difficult to explain within a working memory model that posits an entirely phonological basis of memory storage without also assuming that representations have assistance from long-term memory during recall. Therefore, these results are difficult to reconcile with Baddeley and Hitch's (1974) working memory model but may be able to be explained within an updated version of the model which is outlined in Section 3.8.

\subsection{Connectivity with Long-Term Memory: The Redintegration}

\section{Hypothesis}

The working memory model predicts all items have an equal chance of recall from short-term memory after factors affecting rehearsal such as word length are held constant. The findings of superior recall for words over non-words, common words over rare words, and concrete words over abstract words are clearly incompatible with this view. The effects are instead suggestive of long-term lexical knowledge affecting recall from short-term memory. This brings into question the proposal that long-term memory and short-term memory are distinct, and point to a connection between the two systems, beyond that already identified via word learning.

Hulme et al. (1991) provided an amendment to the original equation of memory span (Equation 2.1) to account for this long-term memory influence. This is given by the equation:

$$
s_{i}=c+r_{i} \tau
$$

Note that for consistency, the notations used in Hulme et al.'s original formula have been substituted with those introduced in Equation 2.1. Again, $s_{i}$ is span, $c$ is a 
constant which corresponds to the intercept of the speech rate recall function (expressed as number of items), $\tau$ is the constant which relates to the slope of this function, measured in seconds, and $r_{i}$ is the speech rate for items $i$. Hulme et al. (1991) interpreted the slope $(\tau)$ is an estimate of the time items take to decay in the articulatory loop, and the intercept $(c)$ as an estimate of the long-term memory contribution. While this allows for the inclusion of long-term memory factors to influence memory span, it does nothing to elucidate the nature of this interaction. An attempt to provide a testable account of the nature of the long- to short-term memory interaction was offered by Schweickert (1993).

Schweickert (1993) described a multinomial processing tree model of redintegration (see Figure 3.1) to explain how long-term memory properties influenced recall from short is an attempt to explain how recall of a single item in a list is achieved by carrying out a series of cognitive processes. These processes can be represented by branches, which stretch from the root of the processing tree to one of the end nodes. The outcome of recall, whether it results in the correct item, an incorrect item, or no item, depends on which terminal node has been reached. According to the model, participants will first attempt to recall an item directly from short-term memory. Failing this, participants will attempt to redintegrate the item using information from long-term memory.

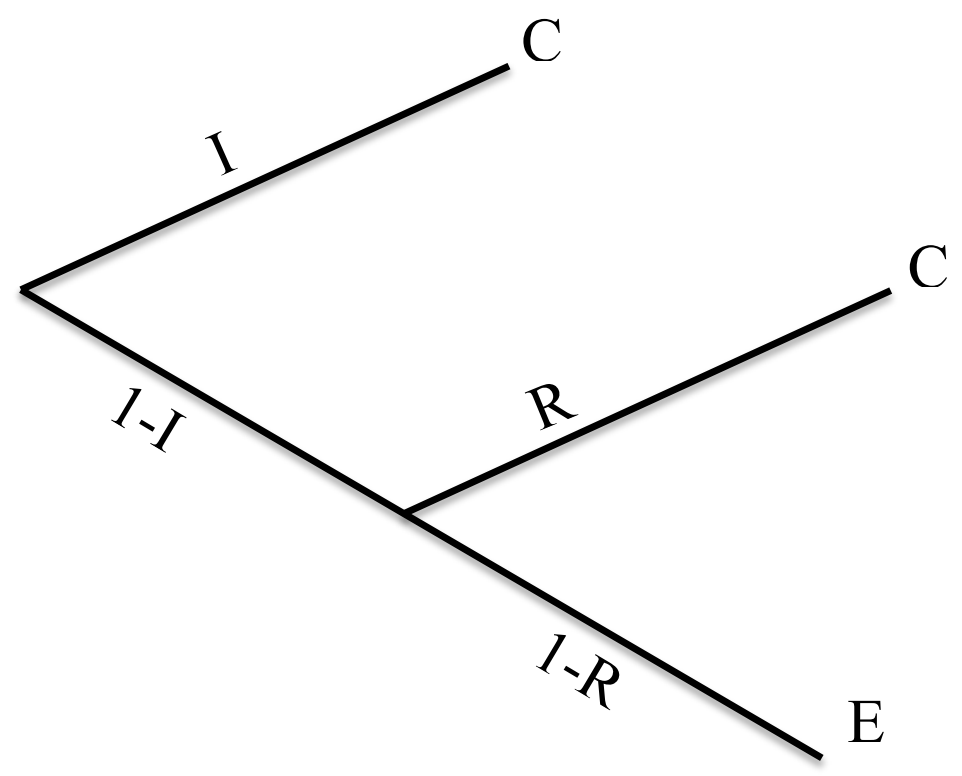

Figure 3.1. Multinomial processing tree model (Schweickert, 1993). If the trace is intact or redintegrated then it will be correct $\mathrm{C}$, or will otherwise be an error $\mathrm{E}$. 
According to Schweickert's (1993) model, an item has a probability $I$ that the item is intact at recall. At probability $1-I$, the trace is degraded beyond the point of direct recall. An attempt to restore information lost from these items will then begin via redintegration. The likelihood of correct recall is given by:

$$
\mathrm{PC}=I+(1-I) R \quad \text { [equation 3.2] }
$$

Where PC is the probability an item will be correct, $I$ is the probability an item will be intact and $R$ refers to the probability that the reconstruction process is successful.

The combined effects of $I$ and $R$ are underadditive. This means that increases on the probabilities of both $I$ and $R$ would lead to a combined effect that is less than the sum of the individual effects (Schweickert, 1993). Items that cannot be output directly will undergo two autonomous processes. In one process, the system attempts to transform the degraded item into a word. This is to account for the finding that words are recalled more accurately than non-words. In the other process, the item is transformed into a string of phonemes. This latter process was included to account for vocabulary learning. In addition, words can be constrained by syntax or context if the items are presented in some meaningful sentence. Schweickert (1993) likens the redintegration process to monitoring one's inner speech for speech errors, and assumes that corrections to speech errors and redintegration of decayed items occur in the same way. The primary difference, according to Schweickert, is that ISR involves a list of items that are already formulated into language, while during speech production some of the parts will have been formulated into language while other parts have not.

The redintegration process is assumed to occur during retrieval, both during rehearsal and immediately before response production (Hulme et al., 1995). Hulme et al. (1999) assumed that the process was separate from the response production processes, including articulation and motor programming. Early views of redintegration also likened the pattern completion process to perceiving speech within noise (Hulme et al., 1997). This was supported by findings that most lexical variables show the same effect on speech perception as they do on serial recall, for instance, 
high frequency items are perceived more easily in noise than low frequency items (Howes, 1957), and are also more likely to be recalled in a serial recall task than low frequency items (Hulme et al., 1999).

\subsubsection{A Dynamic Network Model of Redintegration}

Lewandowsky (1999) created a network model of redintegration as an annex for extant models of memory for order, which did not explicitly identify how items are disambiguated in recall. The model is based on "brain-state in a box" (BSB), an auto-associative network model, created by Anderson, Silverstein, Ritz, and Jones (1977). One of the main properties of BSB is that when it is cued with a starting vector, the output is fed back into the network until a stable state is reached. The stable state, known as an attractor, contains all previously studied items as well as a number of false attractors. The model consists of two stages, an associative stage and a redintegrative stage. The associative stage is modelled as TODAM (Lewandowsky \& Murdock, 1989), although it is also compatible with OSCAR (Brown, Preece, \& Hulme, 2000) both of which will be discussed in Chapter 6 .

In regards to redintegration, Lewandowsky (1999) assumed that presented items are first encoded into BSB. At recall an associative stage takes place, where the memory trace is accessed and at least a portion of the information about an item and its ordinal position is retrieved. This creates a partial response vector (known as $f^{\prime}$ ), which acts as a starting vector for BSB. The second stage, referred to as redintegration, involves the reconstruction of the retrieved information into a complete item and selection of an overt response. If the attractor, $f^{\prime}$ is similar to the vector associated with the presented item $(f)$, then the exact word will be recalled. If $f$ ' falls within a different "basin of attraction" then a different item, either from within the list or not, will be recalled (Lewandowsky, 1999).

Once an item is recalled, it goes through a response suppression process known as anti-learning. This involves reducing the strength of the attractor by giving it negative activation. The amount of negative activation given to an item is dependent on properties of that item, so some may be only partially suppressed. Once recall is complete, the entire list is released from suppression. 


\subsubsection{Explaining the Lexico-Semantic Effects using Redintegration}

According to the redintegration hypothesis degraded traces are restored by comparing the surviving information to items in long-term memory. The lexicality effect occurs because non-words do not have long-term memory representation, and so will not benefit from this process (Hulme et al., 1995). Further, memory is better for frequent words because the memory representation of these words is either better specified or more accessible than that of infrequent words (Roodenrys et al., 1994). When a degraded trace undergoes redintegration, items with more accessible representations are more likely to undergo accurate reconstruction than are items with less accessible long-term memory representations.

In line with Equation 3.1, lexicality and word frequency are assumed to influence the long-term memory parameter, represented as $c$. Specifically, words are assumed to have a value of $c>0$ while non-words have a value of $c=0$. High frequency words are assumed to have higher values on $c$, while low frequency words have lower values on $c$.

In regards to the Schweickert (1993) model (Equation 3.2), factors can influence either the $I$ parameter or the $R$ parameter. Schweickert, Chen, and Poirier (1999) suggested that serial position selectively influences the $I$ parameter with degradation increasing across serial position. Given that word frequency is a longterm memory variable, it can be assumed that this influences the $R$ parameter. Schweickert et al. (1999) note that the model would predict a greater influence of $R$ as the influence of $I$ increases. This would result in a larger frequency effect at terminal serial positions as these items are more degraded than earlier items. This is supported by the literature with Hulme et al. (1997) finding a fanning out of the word frequency effect at later serial positions. Schweickert et al. (1999) suggested that lexicality is also thought to affect the $R$ parameter. If non-words are treated as extremely low frequency words then the predictions made by the model for word frequency should also apply to lexicality. Findings from Saint-Aubin and Poirier (2000) show a greater lexicality effect in the medial positions rather than the terminal serial positions, which is incompatible with this explanation. However, Saint-Aubin and Poirier (2000) also found a ceiling effect for lists of words, and a fanning out of the effect may be evident when a greater amount of errors are elicited. Further, the language familiarity effect 
is also suggested to be an instantiation of the word frequency effect, and can be explained in the same way.

The robust beneficial effect of semantic similarity on item memory can be explained within a redintegration model by assuming that while similar and dissimilar lists are equally affected by decay, the availability of a taxonomic category for similar lists acts as an additional cue for recall (Saint-Aubin \& Poirier, 1999a). Further, redintegration is able to account for the effects of concreteness and imageability by assuming that concrete words have stronger semantic representations, which facilitate reconstruction of these items during recall. Walker and Hulme (1999) noted that word frequency and concreteness effects manifested differently across lists, with the influence of word frequency increasing across serial positions, while the concreteness effect attenuates at the end serial positions. On the basis of this interaction they suggested that separate phonological and semantic redintegration systems were able to contribute to short-term memory. Miller and Roodenrys (2009) challenged this finding by demonstrating that concreteness and word frequency effects showed similar patterns across serial position. They noted that previous findings of a frequency effect that fanned out across serial positions used closed rather than open stimuli sets, with a small number of stimuli presented over a large number of trials. Miller and Roodenrys explained their results within a language based model of shortterm memory. According to these models, lexico-semantic effects occur because of bidirectional links between lexical-phonological and semantic representations of words.

\subsection{The New Working Memory Model: Baddeley (2001)}

Baddeley (2001) revisited the working memory model, partly in response to evidence of a link between short-term memory and long-term memory accrued from decades of experimental work. The initial response of attributing the interface between long- and short-term memory to the central executive was never fully detailed, and it became apparent that the original working memory model was insufficient to manage this and other findings. In response, Baddeley (2001) provided an updated model, which is depicted in Figure 3.2. The new model enabled 
explanation of the long-term memory influence on short-term memory, as well as resolving other issues plaguing the original model (see Baddeley, 2002 for review).

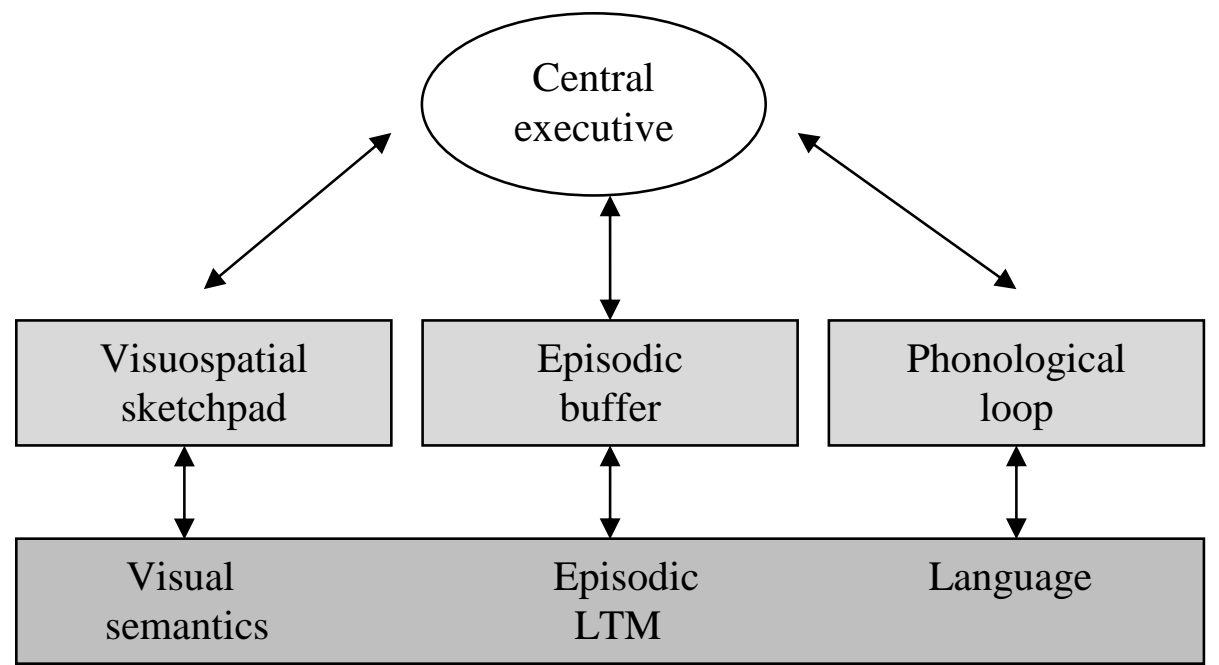

Figure 3.2. Baddeley (2001) revised working memory model. The light grey areas represent fluid systems, while the darker grey represents crystallised systems.

Of consequence in this model is the central box, termed the episodic buffer. This multimodal episodic buffer is the proposed interface between short-term and long-term episodic memory. It is a limited capacity buffer that stores integrated scenes and enables integration between systems with different codes. Baddeley (2002) likened this buffer to the long-term working memory system proposed by Ericsson and Kintsch (1995). With the addition of the episodic buffer, the central executive was redefined as an attention control system. Although the episodic buffer was proposed as a system to link the subsidiary systems, there is no direct link between the episodic buffer and either system. Baddeley (2002) noted that this is because information binding between the subsystems is done by way of the central executive.

In addition to the episodic buffer there is a link between verbal and visual long-term memory, which serves the phonological loop and visuospatial sketchpad respectively (Baddeley, 2002). Importantly, the flow of information is bi-directional, which allows not only for the transfer of information from short-term memory to long-term memory, but also for verbal short-term memory to be assisted by linguistic knowledge.

While the episodic buffer has been purported to account for long-term memory effects on short-term memory, it still has a long way to go toward explaining how this actually works. Burgess and Hitch (2005) tentatively ascribe long-term 
memory effects on item information in short-term memory to the link between the phonological loop and language. Further, the effect of long-term memory on shortterm memory for order was attributed to the link between the episodic buffer and long-term memory. This latter link takes place in the form of a context signal, which is further explained in the Burgess and Hitch (1999) model described in Chapter 6.

A further model, Figure 3.3, was created to include the neural correlates of working memory (Vallar \& Papagno, 2002). According to the model, auditory information has direct access to the store. Visually presented material requires recoding verbally using the rehearsal system. Rehearsal occurs by cycling items through the phonological short-term store and the phonological output buffer (Baddeley, 2005).

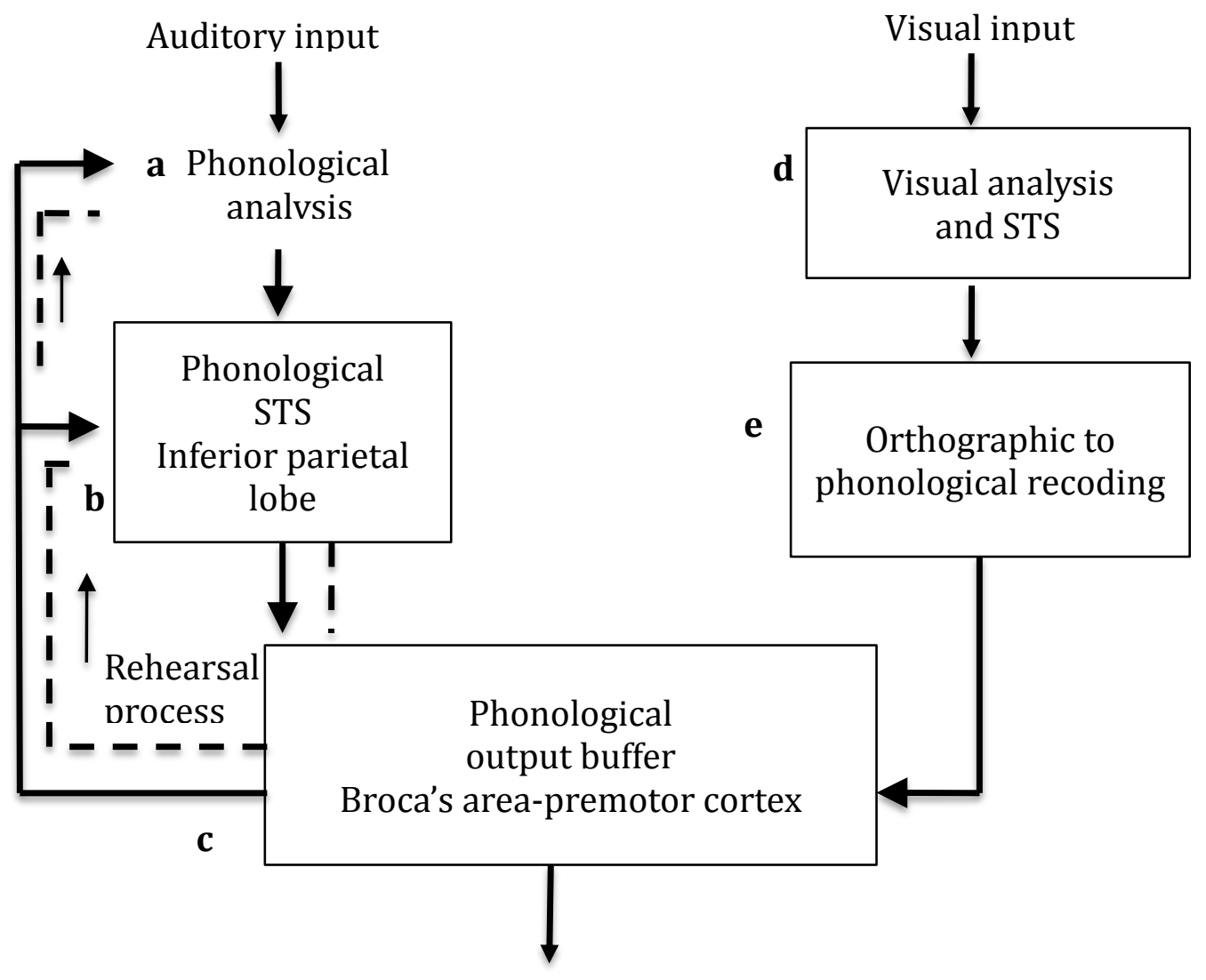

Spoken output

Figure 3.3. Functional model of the phonological loop (Baddeley, 2005). a phonological analysis; $\mathbf{b}$ short-term storage; $\mathbf{c}$ the programming of speech output; $\mathbf{d}$ visual encoding; e Grapheme to phoneme conversion. 


\subsection{Conclusion}

Evidence that lexical and semantic factors influence short-term memory has made the view that long- and short-term memory systems are entirely discrete defunct. As a result, it is now widely accepted that long-term memory plays a role in the retrieval of items from short-term memory, most commonly attributed to a process known as redintegration. In addition to these effects there is also evidence that factors other than the lexico-semantic properties of presented words can influence recall. That is, there is evidence that the long-term memory representation of words, which were not currently in short-term memory, may influence the recall of target items from short-term memory. These neighbourhood effects are indicative of how the flow of activation in the long-term memory system during redintegration influences lexical selection. 


\section{Chapter Four}

\section{Effects of the Lexical Neighbourhood}

\subsection{Introduction}

Lexicality and word frequency effects reflect the influence a word's status in long-term memory has on its maintenance in short-term memory. Accordingly, words are more likely to be recalled from short-term memory than non-words, because words have representation in long-term memory. Further, words used often in language are more likely to be recalled compared with those used less frequently, due to either their greater specificity in long-term memory or more likely due to a high level of co-occurrence between high frequency words. These effects are indicative of how characteristics specific to an individual word can influence recall of that item from short-term memory. There is also evidence that words are accessed relationally in the context of other phonologically similar words (Dirks, Takayanagi, Moshfegh, Noffsinger, \& Fausti, 2001). This is evidenced by the phonological neighbourhood effect and the phonotactic frequency effect. These effects suggest that how frequently certain phonotactic sequences appear in language can influence how likely words that are comprised of these sequences will be recalled from short-term memory. Rather than suggesting that the phonological composition of a word makes it intrinsically easier to recall, the effects are thought to be indicative of how activation is distributed within the mental lexicon. That is, while word frequency and lexicality effects reflect how characteristics of a whole word can influence memory of that item, phonological neighbourhood effects are indicative of how words that are similar in form to a target word can influence the probability that the target will be recalled from memory. 


\subsection{The Phonological Neighbourhood Effect}

\subsubsection{Defining the Phonological Neighbourhood}

Under perfect conditions a stimulus word unambiguously represents a graphic or phonological word (Landauer \& Streeter, 1973). Viewing (or listening) conditions that are deficient will result in an imperfect representation of the stimulus word and may not provide a unique representation of any single word. A phonological neighbourhood is a set of words from which a target word is indistinguishable after a specified amount of phonological information is lost from the stimulus word. More simply, a phonological neighbourhood is a corpus of phonologically similar words.

What defines a phonologically similar word differs somewhat between researchers, although it is generally accepted that two words are phonological neighbours if they share all but one phoneme. In studies of speech perception and production, this typically may be an addition, deletion or substitution of any one phoneme in any position (see, for instance, Vitevitch \& Luce, 1998; Vitevitch \& Luce, 1999; Vitevitch, 1997). In studies of visual recognition processes, phonological neighbourhood membership is defined by the substitution of a single phoneme in any single position (see, for instance, Coltheart, Davelaar, Jonasson, \& Besner, 1977). Roodenrys et al. (2002) adopted this latter definition in their research on neighbourhood effects in short-term memory, primarily to restrict their item pool to single syllable CVC words. According to this definition cat is a neighbour of cap and bat but not at or scat. Both definitions provide almost identical estimations of neighbourhood size, with Roodenrys (2009) finding that the two correlate at .97 . This enables comparison between bodies of research adopting either definition. The current thesis adopts the definition employed by Roodenrys et al. (2002), as the stimulus pool is restricted to words three phonemes in length.

There are two properties of a phonological neighbourhood that are of consequence in cognitive research, the size of the phonological neighbourhood, and the average word frequency of the neighbours (Goldinger, Luce, \& Pisoni, 1989). Words differ from one another by how many neighbours they have. For instance cat resides in a dense phonological neighbourhood with thirty-six neighbours, whereas void resides in a sparse phonological neighbourhood with only six neighbours. 
Findings relating to the influence of the size of a word's neighbourhood are known as phonological neighbourhood, or neighbourhood density, effects, whereas findings relating to the frequency of the words that make up a neighbourhood are known as neighbourhood frequency effects. Neighbourhood density is highly correlated with phonotactic probability, which refers to frequency of segments and sequences of segments in the language (Vitevitch, Luce, Pisoni, \& Auer, 1999). Phonotactic probability is used to examine effects of common sound sequences on the processing of non-words.

Phonological neighbourhoods can be further classified by their clustering coefficient (Chan \& Vitevitch, 2009). This is an index of not only how many phonological neighbours a target word has, but also how many of those neighbours are also neighbours with one another. For instance, bat, hat, and can are all neighbours with cat, but bat and hat are also neighbours with one another while can is not. The clustering coefficient measures the proportion of a word's neighbours that are also neighbours with one another. Words with a large proportion of neighbours that are also neighbours with one another are said to have a high clustering coefficient. The clustering coefficient has been shown to influence speech perception (Chan \& Vitevitch, 2009) and production (Vitevitch, 2008). An example of clustering in the network is shown in Figure 4.1.

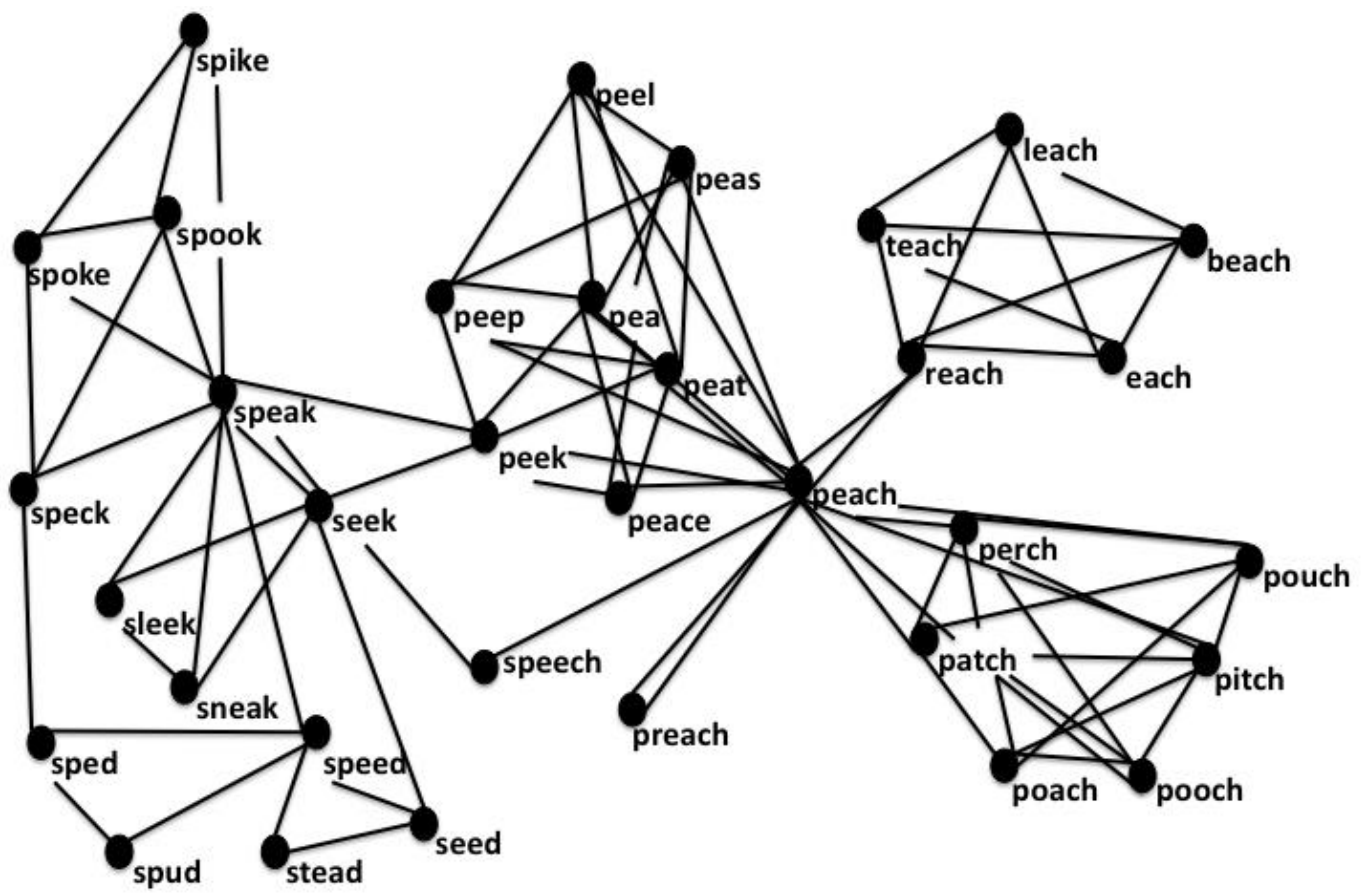

Figure 4.1: Part of the phonological lexicon examined in Vitevitch (2008). Figure is replicated from Chan and Vitevitch (2009). 
Vitevitch's (2008) analysis of the structure of the mental lexicon suggests words are not evenly distributed in phonological space, they are not ordered arbitrarily, but are instead arranged in clusters of neighbouring words.

\subsection{The Phonological Neighbourhood Effect on Speech Perception}

Goldinger et al. (1989) examined the effect of phonological neighbourhood density on the speed and accuracy of speech perception in three tasks including perceptual identification, auditory lexical decision, and auditory word naming. It was hypothesised that presentation of a word would result in activation of other words within the phonological neighbourhood and these words would provide competition for the correct recognition of the target item. It was expected that there would be more competition for words residing in dense phonological neighbourhoods, manifesting as poorer recognition for these items. In line with this hypothesis, the number of words in a target's lexical neighbourhood influenced the speed and accuracy of auditory word recognition with perception more difficult for words from dense neighbourhoods than those from sparse neighbourhoods. The neighbourhood activation model of speech perception was developed where presented traces are compared to possible matches in the mental lexicon. Words with phonemes common to the presented word act as competitors. If part of the word could not be perceived due to noise, then it is more likely that this word will be replaced by one of the competitors. Words from small phonological neighbourhoods have fewer possible matches with the presented trace and so the correct word is more likely to win during the competitive process.

Dirks et al. (2001) replicated the neighbourhood effect on speech perception in both normal hearing and hearing-impaired listeners. Words from small phonological neighbourhoods were recognised more accurately than words from large phonological neighbourhoods in both groups of participants. This provides support for the neighbourhood activation model and suggests that the model can be applied to hearing-impaired populations.

Vitevitch (2002) examined a corpus of naturally occurring slips of the ear, which were collected by Bond (1999). Vitevitch examined the lexical properties of the slips of the ear and examined their neighbourhood characteristics. It was found 
that misperceived words were more likely to come from denser phonological neighbourhoods. This finding supported Luce and Pisoni's (1998) proposition that increases in the density of lexical neighbourhoods leads to increased competition during auditory word perception using a more ecologically valid task.

Chan and Vitevitch (2009) examined the effect of neighbourhood clustering in a perceptual identification task using the clustering coefficient outlined by Vitevitch (2008). While keeping word frequency, familiarity, neighbourhood density and frequency, and phonotactic probability constant, Chan and Vitevitch tested participants on auditory identification of words with either high or low clustering coefficients. The words were presented in white noise and participants typed the word they heard (or a "best guess"). Participants were more accurate on words with a low clustering coefficient than on words with a high clustering coefficient. This lends credence to the theory that it is not only the number of words phonologically related to a target word that influences perception, but also the way that neighbours are interrelated. Chan and Vitevitch (2009) also examined the effect of the clustering coefficient on a lexical decision task. In line with the findings from the perceptual identification task it was found that words with a high clustering coefficient were responded to slower than words with a low clustering coefficient. Further participants were less accurate at responding to words with high clustering coefficients than to those with low clustering coefficients, although this difference was only marginally significant. These results were taken to suggest that not only do the interrelations of neighbours influence the accuracy of perception of a target word but they also influence the time course of lexical access.

In summary, characteristics of phonological neighbourhoods have been associated with effects on auditory word perception in a number of tasks. Most prominently, an increase in the size of the phonological neighbourhood is associated with an increase in response latency and a decrease in accuracy. This has been taken as evidence for lexical competition occurring during perception. The finding has been explained within a number of models of speech perception but has most notably been a springboard for the development of the neighbourhood activation model (Luce \& Pisoni, 1998). 


\subsubsection{The Neighbourhood Activation Model}

In response to their findings of a disadvantage for dense neighbourhood words in speech perception tasks, Luce and Pisoni (1998) proposed a model to map the perception of words (see Figure 4.2). This neighbourhood activation model (NAM) assumes that the presentation of a stimulus activates a set of acoustic-phonetic patterns in memory. This includes the acoustic-phonetic pattern associated with the presented word, as well as other similar patterns. All patterns are activated regardless of whether they correspond to real words, to allow for the recognition of the acoustic patterns of novel words. The patterns of activation that correspond to a word in the mental lexicon will activate a system of word decision units. Once these decision units are activated, they monitor their corresponding acoustic-phonetic pattern. In addition, the decision units' monitor the higher level lexical information pertaining to the activated word, including word frequency information. This separates NAM from other models of memory, such as TRACE (McClelland \& Elman, 1986), which model word frequency as intrinsic properties of the word. As processing proceeds, the phonetic-acoustic pattern that corresponds to the stimulus input will continue to receive activation, while activation levels of patterns that are just similar to the input are reduced. Word recognition is achieved once the word decision unit associated with a pattern of acoustic-phonetic activation passes some criterion (Luce \& Pisoni, 1998).

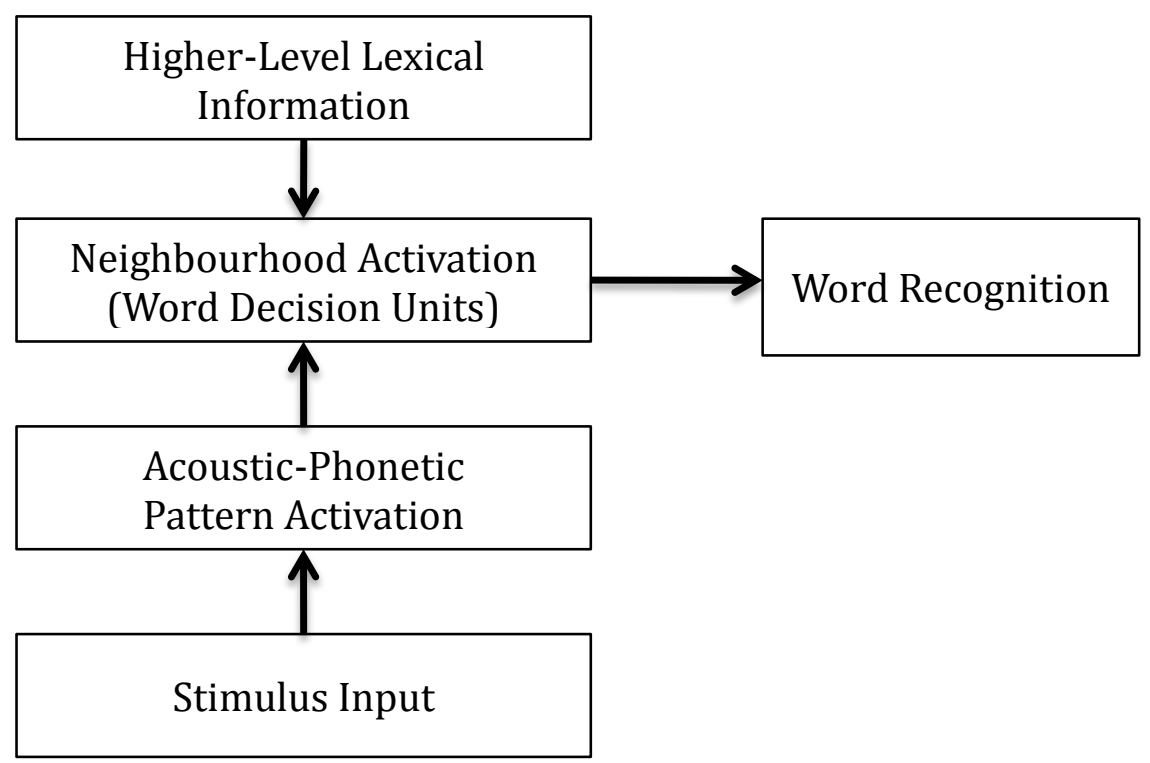

Figure 4.2. Flow chart for the neighbourhood activation model (Luce \& Pisoni, 1998). 
The decision units are also interconnected, which allows for each unit to monitor the overall activity level of the system, including the activity level of other word decision units and their corresponding acoustic-phonetic patterns. Using all of this information, the decision units compute decision values using the neighbourhood probability rule (Equation 4.1). The neighbourhood probability rule estimates the probability of correctly recalling a word based on the number of phonological neighbours and the frequency of said neighbours. The equation for the neighbourhood probability rule is:

$$
\mathrm{NPR}=\frac{S W P \times \text { Freq }_{s}}{\left(S W P \times \text { Freq }_{s}\right)+\sum\left(N W P_{j} \times \text { Freq }_{N j}\right)} \quad \text { [equation 4.1] }
$$

That is, the probability of correctly recalling a word can be given by level of the acoustic-phonetic patterns given by the stimulus word probabilities (SWP) multiplied by the frequency of the stimulus word $\left(\right.$ Freq $\left._{s}\right)$, which is divided by the overall level of activity in the decision system, given by the sum of the neighbourhood word probabilities $\left(\mathrm{NWP}_{\mathrm{j}}\right)$, and frequency information. The frequency information includes both the frequency of the stimulus word $\left(\right.$ Freq $\left._{s}\right)$ and the frequency of the jth neighbour $\left(\right.$ Freq $\left._{N j}\right)$. The probability of the stimulus word (SWP) is calculated by obtaining the conditional probability of each phoneme in the stimulus word using confusion matrices. The SWP is the product of these conditional phoneme probabilities.

While NAM has been effective in explaining effects of phonological neighbourhood density in speech perception, there have been a number of criticisms levelled against the model. Vitevitch and Luce (1999) for instance, provided evidence for both lexical and sublexical levels of processing operating in speech perception. They examined phonotactic probability and neighbourhood density in spoken word recognition using words and non-words. Using a speeded same-different judgement task, the researchers found that correct responses were faster for words from sparse phonological neighbourhoods, but were faster for non-words from dense phonological neighbourhoods. This was taken as evidence for a dual-level processing account of auditory word perception, which they based on Grossberg, Boardman and Cohen's (1997) adaptive resonance model. Vitevitch and Luce (1999) argued that an item's lexical status determines the level of processing. Non-words will be processed at a 
sublexical level and will be subject to effects of phonotactic probability, whereas words will be processed at a lexical level and subject to effects of neighbourhood competition. The lexical level processing of word units overrides any facilitative effect of neighbourhood density occurring at the sublexical processing of these units. Vitevitch and Luce (1999) note that the finding is inconsistent with NAM, which only captures processing at the lexical level, but may be accounted for by a more sophisticated version of the model, called PARSYN, which involves two layers of processing (Luce, Goldinger, Auer, \& Vitevitch, 2000).

A second criticism involves the manner in which items in NAM are arranged. Items in NAM, like other models of speech perception, are arranged in an arbitrary manner. However, Chan and Vitevitch (2009) challenged this assumption by demonstrating that neighbourhood clustering, which is not randomly determined, influences performance. They suggested that this criticism could be resolved within NAM by creating an additional coefficient to allow for the effect of the interconnectivity of word units. Failing this, a new model of the mental lexicon where items are arranged in a non-arbitrary way would need to be described.

\subsubsection{All Neighbours are Equal in Lexical Space}

Research on speech perception has enabled further distinctions to be drawn between models of neighbourhoods where the corresponding phoneme must appear in the same position in both words to consider them neighbours, or models where the shared phoneme can appear in different positions in the two words. This is indicative of a larger issue of whether phoneme identity and position are represented in a unitary manner, or whether they are stored separately. According to the former view, cat is a neighbour of pack as well as cap, while according to the latter view; cat is a neighbour of cap but not pack (Goldrick, Folk, \& Rapp, 2010).

Newman, Sawusch and Luce (2005) examined the possibility that the location of similarities and differences with neighbouring words may have an effect on the level of activation of a word's neighbour, and thus its role as a competitor in speech perception. The proposal arose from predictions made by the cohort model of Marslen-Wilson and Welsh (1978). The cohort model purported that only words commencing with the same initial segment as the target word would compete in word recognition. This proposal had received some early empirical support from studies 
that showed that recognising errors in pronunciation was more successful if the mispronunciation occurred early in the word than when it appeared later in the word (Cole, Jakimik, \& Cooper, 1978).

Newman et al. (2005) examined whether neighbours that mismatch at the onset of the word play the same role as neighbours that match at the onset using a lexical decision task. Non-words were selected that come from one of two groups. The first group was called the cohort and contained words that would be considered large neighbourhood words when only the onset segment was considered, but small neighbourhood words if all possible neighbours were considered. The second group contained words from large neighbourhoods when all possible neighbours were considered, but would be considered small neighbourhood words if only the onset were considered. This was called the full neighbourhood condition. If only the onset of the word plays a role in perception of a word then non-words that come from a large neighbourhood when examining only the onset will be responded to slower or equivalently to the non-words that are not necessarily matched on the initial segment. Alternatively, if the position of the match does not play a role, then the non-word condition based on the phonological neighbours of the whole word will show slower reaction times than the onset-only condition. The results supported the latter hypothesis, with slower response times for non-words that had large phonological neighbourhoods based on all segments. The results were taken to suggest that lexical activation involves the whole word rather than a special influence of the initial segment. Note that this result is inconsistent with the result found in the Vitevitch and Luce (1999) study described earlier. Vitevitch and Luce found a facilitative effect of phonotactic probability and neighbourhood size for non-words in speech recognition, while Newman et al. found an inhibitory effect of neighbourhood size. Newman et al. did not control for phonotactic probability, and while the two are highly correlated, they are not synonymous. This may have resulted in some low phonotactic probability non-words being used in the high neighbourhood density set, which may have resulted in slower processing times for these items. Despite this, the Newman et al. (2005) result provides validation for the working definition of a phonological neighbourhood employed in this thesis, where words can differ by a phoneme in any position. 


\subsection{The Phonological Neighbourhood Effect on Speech Production}

There is ample evidence to suggest that phonologically similar words exert an influence on speech production. Early research focussed on phonological similarity within lists, finding that the speech rate for lists of phonologically similar words is slower to that of lists of phonologically dissimilar words (Sevald \& Dell, 1994). This finding provides support for competitive effects occurring during speech production processes, akin to those already identified in speech perception.

Further research was conducted to examine effects of phonological priming, where phonologically similar words are presented prior to the elicitation of a target word. James and Burke (2000) investigated effects of phonological priming on tip-ofthe-tongue (TOT) states. Participants were instructed to read aloud words that occasionally shared phonemes with a target word and were then asked a general knowledge question known to induce TOT states. Fewer TOT states were elicited when the preceding words shared phonological information with the target word. This was taken as evidence for a facilitative effect of phonological similarity during speech production, which contrasts with the competitive effects found in speech perception tasks, and to the effects found in Sevald and Dell's (1994) speech rate task.

James and Burke's (2000) finding also differs from that of an earlier TOT experiment conducted by Jones (1989). Jones (1989) asked participants to provide the correct word for a given definition. Prior to giving the definition, Jones presented an interloper that either shared the same initial phonological segment with the target word, or was phonologically distinct from the target. Jones found that more TOT states occurred when the interloper was similar to the target word than when it differed. Meyer and Bock (1992) challenge this finding, stating that the stimuli used in Jones' research differed in terms of susceptibility to TOT states. When controlling for this, Meyer and Bock found that phonological relatedness actually facilitated production rather than hindering production.

Vitevitch (2002) examined the effect of phonological neighbourhood density on speech production using the Spoonerisms of Laboratory Induced Predisposition (SLIP) technique. Participants were instructed to internally rehearse word pairs that were rapidly presented on a computer screen. Participants were periodically cued to recall the last two word pairs presented. Word pairs were constructed in a way that 
strongly activates a particular speech plan. For instance, push-big, pig-bull, and pinban activates a p-b speech plan. Participants might then be required to recall beachpalm, which may induce a speech error resulting in the production of peach-balm due to the prior activation of the p-b speech plan. Vitevitch (2002) used the SLIP technique to examine the relative speech errors for large and small neighbourhood words. A significant neighbourhood effect was found, with fewer speech errors for pairs of large phonological neighbourhood words over pairs of words with few phonological neighbours. The research suggests that multiple word forms are activated during speech production and that rather than competing with one another, word forms combine activation to facilitate retrieval of the correct item. Words from sparse neighbourhoods will benefit less from this process, as the production of such words does not activate a large corpus of phonologically similar words.

Vitevitch (2002) replicated this result in a tongue-twister task, and also found that dense phonological neighbourhood words were more quickly produced in a picture naming task than were sparse phonological neighbourhood words. Finally, Allen and Hulme (2006) examined the influence of phonological neighbourhood density on speech production using a number of tasks including definition naming, a delayed repetition task and a measure of maximal articulation rate (number of repetitions per second). There was a significant advantage offered to words from large phonological neighbourhoods in all tasks, with words from large neighbourhoods produced faster and more accurately than words from small phonological neighbourhoods. These experiments support the notion that a word's phonological neighbourhood acts to support production of a target word and also facilitate the timecourse of production.

\subsubsection{Two-Step Interactive-Activation Model of Lexical Access}

The phonological neighbourhood is unique amongst psycholinguistic variables as it has opposing influences on speech perception and speech production processes (Dell \& Gordon, 2003). Other lexical effects, such as word frequency are consistent in their effects on perception and production processes. While the competitive effect of neighbourhood density on recognition in noise is intuitive, given that loss of phonological information potentially makes the item indistinguishable from its 
neighbours, the facilitative effect of neighbourhood density on speech production does not seem as straightforward to explain.

Speech production involves the retrieval of lexical, semantic and phonological information. Theories of speech production assume at least two stages of processing between a concept or semantic representation and graphemic or phonological output. The first stage is driven by the semantic concept and involves the selection of the appropriate word to express the meaning. This is often referred to as the L-level (Rapp \& Goldrick, 2000) or the lemma (Bock \& Levelt, 1994). The second stage is referred to as lexical spell-out and involves mapping the information obtained from the L-level into its constituent phonemes.

Models of speech production can fall under two main categories depending on how they propose activation spreads in the model. There are feedforward models (e.g. Levelt, Roelfs, \& Meyer, 1999) and interactive models (e.g. Dell, 1986). In feedforward discrete models, such as WEAVER++ (Levelt et al., 1999) only lexical nodes activated by the semantic level and selected for output activate the phonological nodes. Because of the feedforward connections, the sublexical level will not influence the process of selection of the lexical node (Baus, Costa, \& Carreiras, 2008).

Interactive models (see Dell, 1986; Dell, Schwartz et al., 1997) assume that there are no lateral connections between neighbouring words. Instead, items are connected to phonologically related words via activation of the constituent phonemes (see Figure 4.3). The model is 'interactive' because connections between the three layers are bidirectional, allowing activation to spread both ways. Connections are also excitatory, which allows information to 'cascade' from level to level (Levelt, 1999). In addition, the nodes are subject to decay and are also influenced by a noise parameter (Dell, Burger, \& Svec, 1997). In Dell's two-step interactive activation model, information at each of the levels is stored using localist representations. That is, nodes represent whole linguistic features such as semantic features, phonological segments, or whole words. 


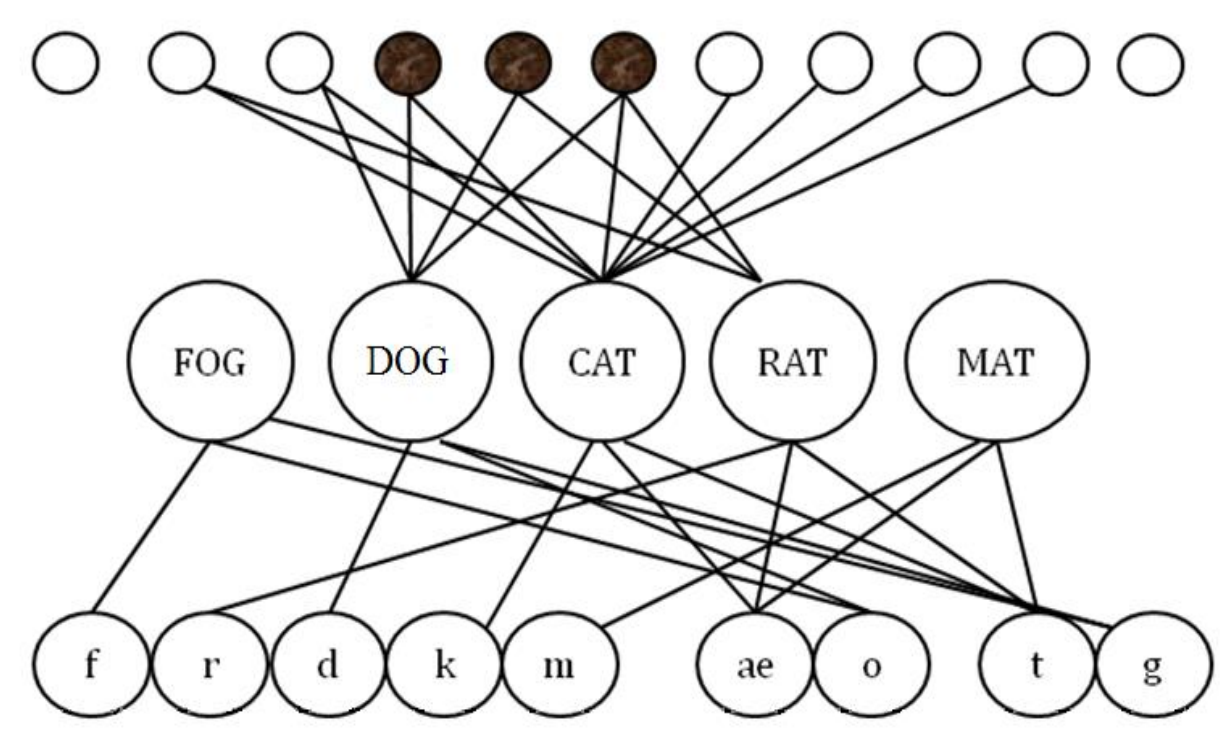

Figure 4.3. The lexical network for the interactive two-step model of naming from Dell, Schwartz et al. (1997). The bottom layer represents the phonological layer, arranged by onsets, vowels and codas. The middle nodes are representative of the lemma or lexical level and the top circles represent the semantic units.

During tasks such as picture naming, a word node is activated via semantic information. The activation of the word node feeds down to the word's phonemes, which pass the activation back to other words via common phonemes. These words become partially activated and pass activation back to the target word through the shared phonemes (Vitevitch, 2002). Lexical selection occurs by choosing the most active item. The observation of a lexical bias in production, that is, non-words tend to be mispronounced as words, validates these models. Baars, Motley and MacKay (1975) examined spoonerisms in experimentally induced slips of the tongue. They found that slips of the tongue tended to result in other words rather than in non-words. However, this was only true when the items were lexical items or when lexical context words were presented. The lexical bias effect is indicative of feedback between the lexical and sublexical level in the production system.

The phonological neighbourhood effect in speech production can also be explained by feedback between these two levels. The neighbourhood effect arises in the model because a greater number of lexical nodes will be activated via common phonemes for words from dense neighbourhoods. These nodes then pass this activation back to the target word via the sublexical level, and the greater pooled activation means that 
words from dense neighbourhoods will be accessed faster and more accurately than words from sparse neighbourhoods (Baus et al., 2008).

\subsection{Effects in the Visual Domain: Neighbourhood Effect on Perceiving Written Words}

The studies described above are specific to words presented auditorily. There is a second body of research describing effects on written words. Yates, Locker, and Simpson (2004) investigated the effect of phonological neighbourhood density on lexical decision using written words. They found that words from large phonological neighbourhoods were responded to more rapidly than words from small phonological neighbourhoods. In addition, participants made more errors with words from small phonological neighbourhoods than with words from large phonological neighbourhoods. This is the opposite of the effect seen with auditory lexical decision. Yates et al. (2004) propose two potential reasons for the discrepancy. First, they note that auditory perception is necessarily serial whereas visual stimuli may be processed in parallel. Visual word forms may activate a word and its phonological neighbourhood almost instantly resulting in facilitation for that word, whereas the serial nature of speech processing may necessarily involve competition to eliminate competitors, resulting in slower and less accurate processing. Second, they note that the auditory stimuli are presented only briefly and the auditory stream dissipates quickly. This may require a heavier reliance on quick selection of an individual word. Visual presentation is less brief, which may enhance the processing of the stimuli.

Ziegler, Muneaux, and Grainger (2003) manipulated phonological neighbourhood density and orthographic neighbourhood density in an auditory lexical decision task. They found that phonological neighbourhood density had the anticipated inhibitory effects, with words in dense phonological neighbourhoods producing more errors and longer latencies in the task. Perhaps more interesting was the effect of orthographic neighbourhood density, which was independent of the phonological neighbourhood effect. Further, the effect of orthographic neighbourhood density was facilitative, which stands in contrast to the inhibitory effects of phonological neighbourhood density. This effect was also larger with dense phonological neighbourhoods while the phonological neighbourhood effect was 
attenuated with dense orthographic neighbourhood words. In further experiments, Ziegler et al. (2003) showed that this orthographic neighbourhood effect was not due to phonotactic probability effects, nor due to a task-specific response mechanism, which monitors global activation in the orthographic lexicon. Instead it provides evidence that orthographic information plays a role in auditory word recognition.

Peereman and Content (1997) examined the phonological neighbourhood effect in speech production for words and non-words. In addition, they differentiated stimuli based on whether they were also orthographic neighbours (so-called phonographic neighbours). It was found that only phonological neighbours that were also orthographic neighbours produced facilitative effects when reading aloud. In addition, only orthographic neighbours that were also phonological neighbours facilitated naming performance. This study was conducted using French words, a language where the mapping between orthography and phonology is highly consistent. In English, this mapping is less consistent and so it may be possible that participants in the French study showed slower response times to items with inconsistent mapping simply because they are unusual.

\subsubsection{Models of Visual Word Perception}

Yates et al.'s (2004) results are explicable from both distributed and localist viewpoints. In distributed models, the system is composed of interconnections between layers of phonological, orthographic and semantic components. Lexical decisions in these models are based on a familiarity judgement of the letter string. More phonological neighbours lead to a higher level of activation for the target word, which in turn leads to a higher probability of correct response. In terms of localist accounts, such as the dual-route cascaded model (Coltheart, Rastle, Perry, Langdon, Ziegler, 2001), lexical decisions can be made based on the amount of activation within a single word or the amount of global activation. That is, words with many neighbours would result in more activation within the entire orthographic system. Either way, this would lead to more correct responses for large neighbourhood words in a visual lexical decision task. However, it would be expected that this would also result in more false positives (incorrectly classifying a non-word as a word) for nonwords from a large phonological neighbourhood. 
Besner and Davelaar (1982) investigated whether the phonological code used to achieve lexical access from print was the same code that underlies maintenance in short-term memory. To explore this, participants performed a visual serial recall task on stimuli that varied with regards to phonological similarity, word length and lexical status. Lexical status was only phonological lexicality as items were either pseudohomophones or non-homophonic non-words. Items were presented under quiet conditions and under conditions of articulatory suppression. As expected, the effects of phonological similarity and word length were present under quiet conditions, but disappeared when concurrent articulation was required. If the task relied solely on a single phonological code, then a lexicality effect should be present under quiet conditions but should be removed under articulatory suppression conditions. Results showed a lexicality effect under quiet conditions, with pseudohomophones recalled better than non-homophonic non-words. However, this effect persevered when concurrent articulation was required, suggesting that two phonological codes operate during visual immediate serial recall. One code, which is not affected by articulatory suppression, allowed for items to access lexical memory. The second code acts as a storage mechanism for items in their order and this is sensitive to effects of articulatory suppression.

\subsection{The Phonological Neighbourhood Effect in Short-Term Memory}

Roodenrys et al. (2002) examined the effect of phonological neighbourhood density on short-term memory using an immediate memory span task. Stimuli were selected based on neighbourhood size and word frequency giving a 2 by 2 frequency by neighbourhood size manipulation. Words were presented auditorily and participant's recall was spoken. Keeping with the premise that redintegration processes operating in serial recall are akin to recognising degraded speech in noise (Hulme et al., 1997), Roodenrys and his colleagues anticipated that the phonological neighbourhood effect operating in serial recall would mimic that arising in speech perception. That is, it was expected that memory span would be greater for small neighbourhood words over large neighbourhood words; and for high frequency words over low frequency words. While the prediction regarding the frequency effect in ISR was supported, the effect of phonological neighbourhood density was contrary to 
expectation. Memory performance was poorer for lists containing small phonological neighbourhood words than for lists of large phonological neighbourhood words. This refuted the suggestion that redintegration was akin to speech perception and the results were instead in line with findings from the speech production literature.

Allen and Hulme (2006) tested the postulation that redintegration is akin to processes underlying speech production by examining the effects of neighbourhood density and word frequency in a number of tasks typically used to assess speech perception, production and short-term memory. They found significant word frequency and phonological neighbourhood density effects on short-term memory, with superior serial recall performance for high frequency words and for words from large phonological neighbourhoods. Effects on speech perception were assessed by looking at reaction times in a lexical decision task and performance on a word identification in noise task. Consistent with the literature, processing was faster for high frequency words and slower for words from large phonological neighbourhoods. Effects on speech production were assessed via definition naming, delayed repetition and maximum articulation rate tasks. Items were responded to faster and more accurately in the definition naming task if they were of a high frequency and from large phonological neighbourhoods. Similar effects were found with delayed repetition, with participants faster at repeating words from large phonological neighbourhoods although this was only significant for highly frequent words. Further, there were significant beneficial effects of neighbourhood density and word frequency on the maximum articulation rate task, with the greatest benefit for large neighbourhood, high frequency words.

Allen and Hulme (2006) aimed to identify the role speech input and speech production processes played in ISR by using performance on perception and production tasks as predictor variables for performance on the serial recall task. They found that definition naming accuracy and word identification in noise were significant independent predictors of recall performance, with definition naming accuracy the strongest predictor. Other tasks were not reliable predictors of recall performance. The results suggest that speech output processes play a significant role in performance on the ISR task. This is consistent with a common mechanism underlying the phonological neighbourhood effect in serial recall and speech production. 
The influence of the lexical neighbourhood on short-term recall is not restricted to well-established adult lexicons, with significant neighbourhood effects found in young children both with typical and atypical language development. Thomson, Richardson, and Goswami (2005) compared phonological neighbourhood effects on ISR performance in typically developing and dyslexic children. Participants were 9-year-old children with a diagnosis of dyslexia, as well as controls equated for age and controls matched for reading level (mean age 7 years). Dyslexic children have an impaired phonological short-term memory system (Roodenrys \& Stokes, 2001), but show normal vocabulary acquisition (Swan \& Goswami, 1997), although it is suggested that representations in long-term memory are less well specified for dyslexic children (Thomson et al., 2005). Due to the impaired phonological shortterm memory system, performance was expected to be worse for dyslexic children than for age-matched controls, although the overall neighbourhood effect should be consistent across groups. The stimuli were selected based on their rime neighbourhood density, but the two stimuli sets also differed in terms of their overall neighbourhood density. Dyslexic children's performance matched that of the reading level matched controls, and both groups' performance was worse than that of the chronological age matched controls. There was a significant effect of neighbourhood density that was independent of group, with better performance for words from dense phonological neighbourhoods than from sparse phonological neighbourhoods. This suggests that the long-term memory influence that supports recall of words from large phonological neighbourhoods occurs in both impaired and normally developing children. The pattern of findings suggests that performance was worse for dyslexic children than for age-matched controls because the long-term memory representations are less well specified for dyslexic children.

To summarise, the phonological neighbourhood effect in short-term memory refers to the facilitative influence a word's phonological neighbours have on the retrieval of a target. This effect is distinguished from the phonological similarity effect, because with the phonological similarity effect list items are members of a single phonological neighbourhood, while with the phonological neighbourhood effect no or few phonological neighbours appear within the same lists, and often not in the same experimental stimuli set. 


\subsection{The Neighbourhood Frequency Effect}

Words differ not only by how many similar sounding words they have, but also by the frequencies of these neighbours (Landauer \& Streeter, 1973). If word frequency plays such an important role in lexical selection during perception, production and short-term memory, it is also likely to influence the degree to which similar items facilitate short-term memory or their relative threat as competitors for recall (Goldinger et al., 1989).

The average frequency of a word's phonological neighbourhood has been shown to be an important variable in a number of cognitive tasks including perceptual identification (Dirks, Takayangi, Moshfegh et al., 2001), auditory lexical decision (Luce \& Pisoni, 1998), and picture naming (Baus et al., 2008). Typically, neighbourhood frequency shows a similar pattern of performance as neighbourhood density, with facilitative effects in speech production tasks (Baus et al., 2008) and competitive effects in speech perception (Luce \& Pisoni, 1998). A similar pattern of results has been obtained in short-term memory, with Roodenrys et al.'s (2002) observation that words with neighbours frequent in the language were more likely to be recalled in ISR than words whose neighbours were infrequent in the language.

Although the two effects operate in a complementary fashion in most tasks, there are some tasks with dissociable effects of neighbourhood frequency and density. Luce and Pisoni (1998) found a neighbourhood density effect in word repetition speed but neighbourhood frequency did not influence this task. In addition, while neighbourhood density influenced performance on the slip-of-the-tongue task in Vitevitch's (2002) experiment, neighbourhood frequency did not have an effect. It is possible that the failure to find a neighbourhood frequency effect in these tasks is due to the size of the effect. As the neighbourhood frequency effect is small, it may not be observable in all tasks, or would require much statistical power to observe.

While neighbourhood frequency does not seem to be as important as neighbourhood density in adults, studies with children reveal that this variable plays an important role in lexical access in children. Newman and German (2002) examined neighbourhood density and frequency in a cohort of 7- to 12-year-old children who presented with either typical language development or atypical language development. They examined lexical access using a picture naming task and a 
sentence completion task. Both groups of children were better at naming words from small phonological neighbourhoods than those from large phonological neighbourhoods, a result that was consistent across the different age groups. This is contrary to what is typically found in the adult speech production literature. With regards to neighbourhood frequency, they found that naming was better for words from high frequency neighbourhoods than from low frequency neighbourhoods. Further, the number of neighbours that are more frequent than the target also had an effect. Neighbours more frequent than the target acted as competitors in the process of lexical retrieval, while those less frequent than the target facilitated retrieval of the target. This is another possible explanation for the inability to find effects of neighbourhood frequency in previous research.

\subsection{Explaining the Neighbourhood Effect within the Redintegration}

\section{Model}

The finding of a facilitative effect of neighbourhood density on short-term memory forced the abandonment of the theory that redintegration was akin to perceiving speech in noise. Roodenrys et al. (2002) proposed amendments to the redintegrative theory, drawing from interactive activation models of speech production (i.e., Martin et al., 1999; Dell, Burger, \& Svec, 1997). This type of model is characterised by separate input and output phonological buffers that are connected to input and output nodes in long-term memory. Martin et al. (1999) provided a schematic interpretation of this system (see Figure 4.4). Production involves first semantically encoding the concept to be conveyed. The semantic node is then used to activate the appropriate lexical representation, which is then encoded phonologically to allow for articulation. In speech perception this sequence is reversed, with acoustic pattern first encoded phonologically. This phonological representation is then used to activate the appropriate lexical entry followed by the corresponding semantic information. 


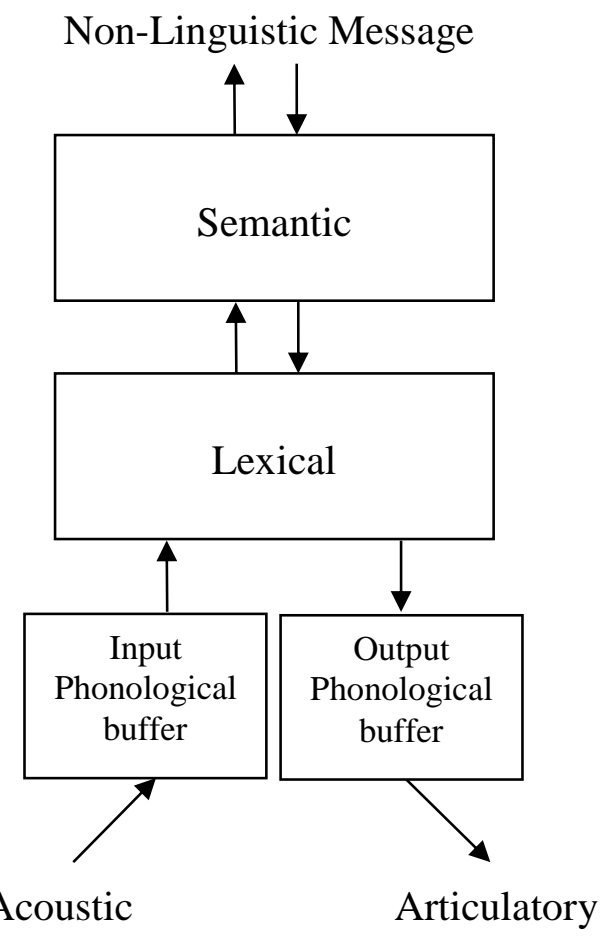

Figure 4.4 Martin et al.'s (1999) levels of representation in speech perception and production. The phonological layer is divided into separate input and output phonological buffers

As the debilitating influence of large phonological neighbourhood size on perception is attributed to the input phonological buffer, it is likely that the facilitative effect observed in speech production and short-term memory is due to the output phonological buffer. Accordingly, redintegration-like processes used to select an appropriate lexical form may function differently in the output buffer compared with the input buffer.

To account for these facilitative neighbourhood effects, Roodenrys and his colleagues proposed a new concept of redintegration that operates on the output side of the model. This was built on the trace decay plus rehearsal approach where items in short-term memory are subject to decay, which can be offset by rehearsal. According to this view, decay may result in the loss of one or more phonemes from each item, leaving them as remnants of the presented word. These traces are recovered from the short-term memory system during recall, and are subject to a redintegration processes in an attempt to recover the lost information. During redintegration, the remaining phonemes are used to activate output phonemic nodes in long-term memory. The activated nodes then pass activation up to the L-level or lemma level, where it activates corresponding word nodes. This activation is returned to the phoneme level 
and activation reverberates in this manner until a stable representation is achieved. An artefact of this process is that all words containing the activated phonemes will themselves be activated to some extent. Words that have more phonemes in common with the trace word will be activated to a greater extent than words that have fewer common phonemes. As a result, more supportive activation will be received by the phonemes from these words and passed on to the target word. The most active word at the end of this process will be recalled so long as its activation exceeds a threshold. Presumably this word will also be that which shares all and only the common phonemes with the recovered trace. Words with a large number of phonological neighbours will be recipients of a greater amount of activation during this reverberatory process, rendering them more active and thus more easily accessed for recall. Words that do not exceed the threshold following redintegration will be unrecoverable during recall. As words from small neighbourhoods receive less supportive activation, these words are less likely to exceed threshold and thus less likely to be recalled.

This instantiation of redintegration is also able to account for the finding that words from large phonological neighbourhoods are more likely to be substituted for a phonological neighbour than are small neighbourhood words. This may happen if too much phoneme information is lost prior to redintegration making selection of the correct word from a number of candidates impossible. For instance, if the phoneme $b$ is lost from bat then it would be impossible to ascertain whether the correct word was bat, cat, sat, or a number of other contenders. In this case, a number of different factors may help to determine which item is selected for recall including retroactive interference (i.e. participant may have been presented with the word ball either earlier in the list or in a previous list, making bat most active either by semantic associations or some residual activation remaining in the $b$ phoneme) and word frequency. Roodenrys et al. (2002) suggested that word frequency may play the more important role here, with the finding that words that erroneously replace target words are not only likely to be phonological neighbours of the word, but they are also likely to be of a higher frequency than the target word. 


\subsection{Phonotactic Frequency Effect}

Phonotactic frequency or phonotactic probability is an index of the frequency with which sounds are combined in language (Vitevitch et al., 1997). Words with high phonotactic frequency such as bell are made up of sounds that appear frequently in the language, and also have combinations of sounds that are frequent in the language. Words such as watch have a low phonotactic frequency, meaning that their sounds and the combination of sounds within the words are quite uncommon in the language (Acheson \& MacDonald, 2009b). As stated earlier, phonotactic probability and neighbourhood density are highly correlated (Vitevitch et al., 1999).

Gathercole, Frankish, Pickering, and Peaker (1999) examined the influence of phonotactic probabilities on serial recall in 7- and 8-year-old children. They examined recall performance on monosyllabic consonant-vowel-consonant (CVC) words and non-words that differed in their phonotactic frequencies. There were four list conditions including words, high probability non-words, low probability non-words, and very low probability non-words. They found a typical lexicality effect when phonotactic probabilities were held constant across stimuli. When examining nonword recall, it was found that high probabilistic non-words were recalled more often than low probabilistic non-words. This was taken as evidence for a sublexical contribution to recall as well as a lexical contribution to recall performance.

Roodenrys and Hinton (2002) examined whether Gathercole and colleagues' (1999) findings were due to a confound with neighbourhood density. Roodenrys and Hinton (2002) examined serial recall performance of non-words that differed in phonotactic probability but did not differ in the number of phonological neighbours. If the effect observed in Gathercole et al. (1999) was due to an effect of phonotactic probability as claimed, then it would be replicated in this study. Roodenrys and Hinton found no effect of phonotactic probability after holding neighbourhood density constant. In a second experiment, Roodenrys and Hinton (2002) examined the effect of phonological neighbourhood density while an aspect of phonotactic probability, biphone frequency, was held constant. They found a significant effect of neighbourhood density after controlling for phonotactic frequency. They concluded that studies showing effects of phonotactic probabilities were likely to be actually 
observing effects of phonological neighbourhood density, and that it is the density variable that influences short-term memory.

Thorn and Frankish (2005) noted that both Gathercole et al (1999) and Roodenrys and Hinton (2002) calculated biphone frequency based on the frequency of occurrence of $\mathrm{CV}$ and VC pairs in CVC words in the CELEX database. In keeping with the Luce, Pisoni, and Goldinger (1990) definition of a phonological neighbour, there are three types of phonological neighbour. These include the $\mathrm{CV}_{-}$and _ VC neighbours already captured by Gathercole and her colleagues and by Roodenrys and Hinton, but also include C_C neighbours. For neighbourhood size to be manipulated while controlling for biphone frequency, the large neighbourhood cohort must necessarily have more C_C neighbours than the small neighbourhood group (Thorn \& Frankish, 2005). This introduces confounds between biphone frequency and C_C neighbours. Thorn and Frankish examined whether Roodenrys and Hinton's result was due to confounds by replicating their experiment while controlling for neighbourhood type. They found a significant effect of biphone frequency when controlling for neighbourhood type, as well as a significant effect of neighbourhood size when controlling for biphone frequency. This suggests that the long-term memory contribution to short-term recall of non-words is not entirely lexical, but also relies on knowledge of the phonotactic structure of language.

\subsection{The Role of the Lexical Neighbourhood in New Word Learning}

Recently, researchers have been interested in the role the phonological lexicon plays in the learning of new words. Research suggests that learning a word does not immediately entrench it within a neighbourhood. According to Leach and Samuel (2007), learning occurs in two distinct stages, the first stage involves lexical configuration, which includes all of the known aspects about a word including phonology, pronunciation and semantics, and the second stage involves lexical engagement, which is an indication of how words interact with other words. According to Leach and Samuel, the learning of these two types of representations occurs at different stages of the learning process. Repeated exposure to a word is enough to enable word learning at the lexical configuration level of processing, and occurs quite quickly. Lexical engagement requires activation of similar word forms in 
the mental lexicon. Repeated exposure to a word, and repeated activation of similar word forms, enables lexical engagement, which means that the word will be solidified in the mental lexicon (Hoover, Storkel, \& Hogan, 2010).

The size of the phonological neighbourhood has been shown to influence new word learning. In a study by Hoover et al. (2010), 3- to 5-year-old children learnt nonwords that varied in terms of their phonological neighbourhood density and phonotactic probability. Exposure to the non-words was provided via a narrative paradigm where non-words were matched with pictures. Word learning was examined using a picture naming task, which was administered immediately after the story and again after a week delay. There was an interaction between phonotactic probability and neighbourhood density. Specifically, uncommon sound sequences triggered word learning better than common sound sequences, but this was only true for non-words from sparse phonological neighbourhoods. They proposed that learning for rare sequences occurred earlier and so fewer presentations were required. Further, dense neighbourhood non-words were learned better than non-words from sparse neighbourhoods but this was only true for common sequences. This suggests that more enduring long-term traces are created for these items. This is presumably because the reciprocal activation of common word forms enabled the non-word to stay in memory for longer, which facilitates learning. This may be because the new word forms links with other common words during lexical engagement. The many links with similar sounding words solidifies the representation in memory. Storkel, Armbrüster, and Hogan (2006) replicated this finding in adult word learning. These results indicate that lexical neighbours assist in integrating a new word into the mental lexicon, both in children and in adults.

\subsection{Conclusion}

Literature indicates that words that reside close to a target word in lexical space influence the processing of a target in a number of cognitive tasks. Or as Dell (1999), more eloquently noted it is "impossible to sneak into the lexicon and take out the word you want without all the neighbours getting excited". These results are indicative of spreading activation to neighbouring items on presentation of a target item. In speech perception this hinders recognition of a word, while in speech 
production and short-term memory spreading activation assists correct production and correct recall. The phonotactic frequency effect reveals that this spreading of activation occurs at a lexical and at a sublexical level.

While the neighbourhood effect on perception and production tasks can be explained within existing cognitive models, the neighbourhood effect on short-term memory requires further clarification. Maintaining an item in short-term memory involves two complementary processes, remembering the items and remembering their sequential order. It is unclear from previous research whether phonological neighbourhood density influences memory for items, memory for order, or both. Lexical variables such as word frequency had been shown to influence item memory but not order memory (Poirier \& Saint-Aubin, 1996). On the basis of these results the phonological neighbourhood effect was explained within existing models of shortterm memory using the redintegration hypothesis. Results from Roodenrys et al. (2002) complicate this explanation by showing an influence of phonological neighbourhood density on memory for order. This finding necessitates an examination of models of memory for order to ascertain how a long-term memory variable could influence order. The following chapter will provide an overview of memory for order, and will outline research examining lexical effects on memory for order. 


\section{Chapter Five}

\section{Short-Term Memory for Order}

\subsection{The Problem of Serial Order}

While the mechanisms required for the maintenance and retrieval of items in memory has a rich history in the experimental and theoretical sphere, it is the internal representation of how items relate to other information that is important in everyday life. It is rare that we would be required to retain an item in isolation in any real world situation; rather, we need to remember sequences of items, often in their serial order. For instance, in placing a phone call, it is insufficient to remember only the digits of the telephone number; instead, one must also remember the order of the digits.

In addition to intuitive uses of maintenance of order information, there is also experimental evidence that reveals order memory as important for higher cognitive process such as vocabulary acquisition (Gupta \& MacWhinney, 1997) and language production (Dell, Burger, \& Svec, 1997). Majerus, Poncelet, Greffe, and Van der Linden (2006) attempted to elucidate the nature of the short-term memory contribution to new word learning. They sought to identify whether item information is sufficient to generate a novel form in long-term memory, or whether it is the serial order component that is necessary for language development. Majerus and his colleagues examined phonological memory in 4- to 6-year-olds using tasks that separated out item and order memory. They found that both item memory and order memory were independent predictors of vocabulary knowledge. Specifically, order memory predicted vocabulary knowledge in 4- and 6-year olds, but was not a significant predictor in 5-year-olds. Item memory was not a significant predictor in 4and 6-year-olds but was a significant predictor in 5-year-olds. Majerus et al. (2006) interpreted their findings as evidence that it is the serial order component of shortterm memory that is important in vocabulary learning. In addition, they note that while serial order memory is a predictor for language development, by around 5-years of age, a developmental shift occurs where short-term memory performance also 
becomes dependent on vocabulary knowledge (see Metsala, 1999). Leclercq and Majerus (2010) replicated this role of short-term memory for order in vocabulary development using a longitudinal design using 4- to 5-year-old children. Further, Perez, Majerus, and Poncelet (2012) demonstrated that short-term memory for serial order, but not item memory, was an important determinant of early reading acquisition in a longitudinal study using 5- to 6-year-old children. These results implicate the role of short-term memory in language development, and further identify that it is the maintenance of serial order that is key in learning new words and reading acquisition. Majerus, Poncelet, Elsen, and Van der Linden (2006) extended these findings to word learning in adults. They presented phonotactically legal nonwords in a paired associate word/non-word learning task. Non-words were paired with words during a learning phase and participants were required to repeat the nonword when cued with the word. The researchers compared learning performance with a serial reconstruction task, which is designed to assess order memory, and with item errors observed in a serial recall task. Non-word learning was associated with performance on the serial reconstruction task but not with the serial recall task. This implicates the role of memory for order in new word learning.

Not only is memory for order imperative for day-to-day functioning at many different levels; it also seems to occur with little error. For instance, remembering a seven-digit phone number, or more commonly a ten-digit mobile number, occurs with few permutations of the digits despite their seemingly arbitrary arrangement (Marshuetz, 2005). On a different level, speech, which involves the correct recall of a sequence or sequences of phonemes, occurs with very little error despite the speed of ordinary articulation. Lashley (1951) dubbed this seemingly innate ability to recall items in their serial order "the problem of serial order", and while extensive theoretical and experimental work has been conducted since, there is still no clear agreement on exactly how serial order is achieved.

\subsection{Two Things to Remember: Item and Order Memory}

Two things must be remembered to accurately recall a sequence of items: (a) the item information, which for words typically refers to the phonological, lexical, and semantic content, and (b) the sequential order of the items. Whether item and 
order information utilise the same processes, or whether they involve distinct operations, is a source of contention amongst researchers.

An early argument for separable processes noted instances in ISR where participants appeared to remember the item information but not the order information. This resulted in transpositions or 'swaps' between items. For instance, $A, B, C, D$, being recalled as $A, C, B, D$, with the middle two items transposed. While this is often seen to be indicative of an order error, some researchers have argued that these are actually item errors (i.e., Conrad, 1965). According to Conrad (1965), decayed traces may be confused with acoustically similar items and this, coupled with participants' reluctance to repeat items in recall could result in an apparent swap of items. Thus rather than being a swap in the order of items, $B$ was mistakenly recalled as $C$, and $C$ mistakenly recalled as $B$. Baddeley (1986) found evidence for this assertion via an examination of phonological similarity in lists that alternated distinct and confusable items. He found superior performance for distinct items, while phonologically similar items tended to transpose with one another, resulting in saw-tooth serial position curves. This was taken to suggest that phonological similarity did not affect order information, but was instead an effect of item information.

Bjork and Healy (1974) refute Conrad's argument that order errors are actually item errors. They note that Conrad's estimation of acoustic confusion errors was inflated because he only examined cases where exactly two of the six items presented had transposed. This reflected only a small number of the order errors made overall (around 17\%). To determine whether Conrad's assertion that item and order information were represented in a common store, Bjork and Healy examined acoustic confusability in four item lists where between 0 and 2 of the letters were confusable. They demonstrated that acoustic confusions were no more likely to occur than by chance. Bjork and Healy also examined whether item information and order information are lost at the same rate in the store, as would be predicted by a common item and order mechanism. By varying the retention interval, they found that participants made frequent item transposition errors at even the shortest retention interval even though item recall was perfect. Their result was consistent with Murdock (1967), who found that the likelihood of making a transposition error increased as the retention interval increased. These results suggest that item information and order information are stored separately, and that order information is lost faster than item information. 
Further evidence for separate processes has been garnered through an examination of serial position curves. These allow for a graphical examination of performance across serial positions. If item and order memory use the same processes, it is expected that performance on tasks assessing item and order information should show similar patterns across serial positions. Donaldson and Glathe (1969) examined serial position curves for item and order memory using probe tasks with a single digit probe for item memory and pairs of digits for order memory. In the single digit task, the participant only needed to indicate whether the probe appeared in the target series. In the order probe task, the participant needed to indicate whether the pair of digits appeared in the same order in the target list as in the probe. Sharper bowed serial position curves were found for the paired digit probe task over the item probe task indicating that order and item errors are due to dissociable processes in memory. Further, Healy (1974) conducted an experiment where particpants performed either an order only recall task or an item and order recall task. In the order only task, participants were presented with permutations of only four letters. In the item and order task there were twelve letters, which were presented in four item lists. As Healy predicted, serial position curves were more bowed for the order only task over the item and order task.

In two separate experiments, Sternberg $(1966,1967)$ examined item and order memory using an item probe task to assess memory for item information and a searching for next task to assess order memory. In both tasks participants were given a list of between 3 and 7 items (e.g., '3, 8, 5, 9') followed by a probe (e.g., ' 8 '). In the item probe task, participants simply had to respond either "yes" or "no" depending on whether or not the probe was present in the list (e.g., 'yes'). In the searching for next task participants needed to respond saying aloud the item that occurred immediately after the probe (e.g., ' 5 '). Sternberg (1967) looked at the reaction time slope relating to the number of items in the list and found that the slope relating set size to the number of items in the memory set was over seven times steeper in the searching for next task than it was in the item probe task. Sternberg concluded that different mechanisms are used for the retrieval of order information and item information. Specifically, Sternberg proposed that item recall involves a fast scanning process whereas order recall involves a slower checking process.

Further support for a separate systems view comes from evidence of dissociable encoding processes for item and order memory. Evidence of this sort 
came from a study by McElree and Dosher (1993). They applied a speed/accuracy procedure to item recognition and order recognition tasks. In the order recognition task, participants were presented with a string of letters and were then probed with two items that appeared within the set. Participants were to indicate which of the letters was presented more recently. In the item recognition task, participants were presented with a string of letters and then at test were presented with a letter from the set and a letter that was new. Participants needed to indicate which letter had been presented in the set. By varying the response time deadline, McElree and Dosher found that the process for encoding order information was more laborious than that required to encode item information. Further, Henson, Hartley, Burgess, Hitch, and Flude (2003) demonstrated that finger tapping selectively disrupted order memory while leaving item memory unaffected. These differences in the processes involved in encoding item memory and order memory are suggestive of a separation between item and order memory.

Neuropsychological evidence has also been used as support for this demarcation. Kesner, Hopkins, and Fineman (1994), for instance, compared patients with bilateral, left, or right damage to the prefrontal cortex to age-matched controls on performance on a number of tasks that tested item and order memory for verbal information, spatial information and abstract pictures. They found that all patients had difficulty with abstract and word order tasks as compared to the item tasks, while only bilateral and right prefrontal damage had difficulty with the spatial order task. This indicates that memory for order is dependent on the prefrontal cortex, while item memory is not. Further, Attout, Van der Kaa, George and Majerus (2012) examined serial recall and serial reconstruction performance of two patients that presented with different areas of neurological damage that related to different profiles of cognitive impairment. One patient (MB) presented with difficulties maintaining item information with intact order memory and another (CG) with a specific deficit in maintaining order with preserved item memory. As expected, CG showed impaired performance on the serial reconstruction task with normal performance on serial recall. Conversely, MB showed performance levels similar to controls on serial reconstruction, but impaired performance on serial recall. This double dissociation is evidence for a distinction between item and order memory.

Majerus (2009) proposed that these and other findings were best handled by hybrid verbal short-term memory models where item information is temporarily 
represented within the language system and order information managed by a separate, specialised short-term memory system. A number of current models of memory assume separate item and order processing, although not all ascribe to the hybrid psycholinguistic model suggested by Majerus. These models will be discussed in Chapter 6.

The literature supporting separate processes for item and order memory is compelling, and this is reflected in the theoretical development of memory models. Despite this advancement in knowledge, research investigating long-term memory effects on short-term memory continues to examine effects using serial recall, a task that is inadequate for separating item and order memory.

\subsection{Inadequacy of the Immediate Serial Recall Task}

As identified earlier, for a response to be coded as correct in ISR it must be the correct item in the correct position. While item and order information both contribute to the generation of a correct response, traditional scoring in ISR is unable to separate the relative influence of these two types of information (Serra \& Nairne, 1993). As such, a measure of absolute performance on this task is insufficient to gauge memory for order. A number of separate analyses may be conducted to assess order memory, including an analysis of order errors and an examination of the proportion of order errors to the overall level of item recall.

\subsubsection{Analysis of Order Errors}

A breakdown of errors within serial recall can provide information about order memory. Conrad (1964) noted that both the number of errors and the "forms of malfunction may provide a short cut to an understanding of structure" (p. 83). Order errors are classified based on either the distance between an item's input position and its output position, or the type of order error. The distance of movement can be assessed through an examination of error gradients, which chart the movement of items from their input position. Error gradients typically show that if item $i$ is not recalled in its correct position $j$, it is more likely to be recalled in position $j-1$ or $j+1$. 
That is, erroneously recalled items are likely to be recalled close to their input position. This is known as the locality constraint (Henson et al.,1996).

In an analysis of the type of order error, reference is made to the direction of movement, with little importance given to the absolute distance between the input and output positions. Typically, order errors can be classified by whether they are transposition errors or whether they occur in conjunction to an item error such as an omission. Transposition errors occur when any two items swap positions, for instance $A, B, C, D, E, F$ given as $A, C, B, D, E, F$. Further, there is a distinction between adjacent transpositions, which occur between neighbouring items, and remote transpositions which occur between items further apart. Again, transpositions are most likely to occur between two neighbouring items, with the chance of transpositions diminishing as the distance between two items becomes greater (Murdock \& vom Saal, 1967). With regards to the direction of movement, errors can be classified as to whether an item was brought forward in the list, which is known as an anticipation error, or whether it was recalled later than its original position, known as a postponement error. Anticipation errors can occur due to an omission at an earlier position, $A, B, C, D, E, F$ given as $A, C, \ldots, D, E, F$, or may occur in conjunction with a postponement error $A, E, C, D, B, F$. Postponement errors may also occur in conjunction with an omission error or with an anticipation error.

The above analyses are based on whether items are recalled in their absolute serial position. That is, given the list $A, B, C, D, E, F$, if $B$ is recalled but is not recalled in the second serial position it is regarded as an order error. A more complex examination of order errors may be conducted on the proportion of recalled items that maintain their relative position within a list (Nairne \& Kelley, 2004). According to this analysis, $B$ would be regarded as maintaining its correct serial position in the above list so long as it was recalled after $A$ and, depending on the criterion used, before $C$. Analyses based on relative serial positions are often conducted on how many pairs are recalled in the correct order. For instance, $A, B, C, D, E, F$, given as $A$, $B, D, F, E, C$, has five pairs recalled and of these, three pairs are recalled in their correct relative position, $A$ and $B, B$ and $D$, and $D$ and $F$. So for this list, three out of five pairs were recalled correctly in order (Asch \& Ebenholtz, 1962). However they are analysed, order errors are predictable in their frequency of occurrence and their type. Because of this, order errors can be a useful indicator of the underlying structure of memory. While an analysis of the order errors emitted during serial recall is useful 
to determine the mechanisms of order memory, a confluence with item errors cannot be ruled out.

\subsubsection{Conditional Probabilities: Immediate Serial Recall Task}

Interpretation of a simplistic analysis of order errors as an influence of a variable on order information is only possible if it can be assumed that item recall is consistent across conditions. As it has been shown that psycholinguistic variables affect the level of item recall, a straightforward analysis of order errors can be misleading. This is because an item can only be reported in the wrong order if the item information is sufficiently recovered, and similarly, items that cannot be recalled cannot be reported as an order error. Therefore conditions that enjoy better item memory will also be more likely to show order errors. To assess memory for order, confounds with item memory can be reduced by controlling for the absolute level of item recall using conditional probabilities.

Conditional probabilities are extracted from the data by scoring responses using both a free recall criterion and a correct-in-position criterion. Conditional probabilities are then calculated by dividing the total number of order errors by the total number of items recalled regardless of serial position (the free recall score) (Murdock, 1976; Saint-Aubin \& Poirier, 1999a). This method acts to equate item recall level across conditions, which allows for a more accurate comparison of the relative number of order errors between conditions. Higher conditional probability scores indicate more order errors per number of items recalled and are suggestive of poor order memory for these items.

The preponderance of research on memory for order uses some kind of a conditional probability analysis. This is despite the fact that serial recall necessarily requires memory for both item and order information and thus any post hoc method of separating item and order memory has the potential to be inaccurate. The following tasks aim to remove this item component by presenting items at both presentation and test. 


\subsubsection{Serial Order Recognition}

In serial order recognition, also known as matching span, participants are presented with a sequence of items and then are re-presented with the same items at recall. Participants are asked to recognise whether a sequence was presented in the same order as the original list (cat, jug, win... cat, jug, win) or in a different order (cat, jug, win... cat, win, jug). The judgment may involve the entire sequence or just a subset of the sequence. As participants do not need to explicitly state the items, this task is argued to reduce the memory load required for the items and should be a more accurate representation of memory for order. Gathercole, Service, Hitch, Adams, and Martin (1999) devised the task as a way to address the argument that traditional shortterm memory tasks such as ISR provide an underestimation of children's short-term memory capacity as they involve accurate execution of speech-motor planning which may be underdeveloped in children. As such, while the task requires that the items be maintained in short-term memory, it is argued that it by-passes the speech production system. As serial recognition circumvents speech output processes, it may also bypass redintegrative processes operating during the ISR task, as only having partial information of the list items at recall will not impede performance. As such, it may be used to investigate effects of linguistic variables over and above that attributed to redintegration effects on item information. This task has been used to examine the effects of a number of long-term memory variables on short-term memory for order including lexicality (Gathercole, Pickering, Hall, \& Peaker, 2001) and concreteness (Walker \& Hulme, 1999).

\subsubsection{Order Reconstruction}

Order reconstruction involves presenting participants with lists of items, and then presenting the words again in a random order. Participants are asked to place the items back in the order they were presented. As with recall, reconstruction can be serial, which requires participants to commence reconstruction with the first item presented and continue sequentially, or can be free reconstruction of order, where participants can begin reconstruction at any point in the list (Neath, 1997). As the requirement for recalling item information is removed, this task is assumed to be a process pure assessment of memory for order (Whiteman, Nairne, \& Serra, 1994). 
This assertion is ambitious, given that it is impossible to remember the order of an item if the item information has been completely lost, an argument similar to that made by Neath (1997). Instead, the task can be regarded as a more accurate assessment of memory for order as participants can perform the task fairly accurately on partial item information. As items are presented at output, redintegration is not required, as any degradation of the internal representation of the item can be "cleaned up" using information given at recall. Majerus, Poncelet, Elsen and Van der Linden (2006) provided evidence that reconstruction is a test of memory for order by showing that the task correlates with an analysis of order errors in serial recall but not with item errors.

\subsubsection{Other Measures of Memory for Order}

A number of other methods to assess memory for order have been used in the literature. Healy (1974) reduced the requirement to remember item information by presenting the same items in a different order from a limited pool of stimuli. If participants are able to anticipate the items they will be required to recall the task is reduced to remembering only the order of the items.

Nairne and Kelley (2004) present a process dissociation framework for separating item and order information. This framework involves comparing task performance on a condition where both item and order are supposed to facilitate performance, called an inclusion task, and a condition where the two processes act in opposition, known as an exclusion task. In terms of the item/order distinction, the inclusion condition would be serial recall, as any increase in item memory $(I)$ and in order memory $\left(O_{R}\right)$ would result in a facilitation of performance. In the exclusion condition, participants are asked to recall all list items except the item presented in position $X$. In this instance, recall of the item that appeared in position $X$ would only occur if the item information had been retained but the order information lost. The probability of this is $\left(1-O_{R}\right)$. Thus, the probability of recalling the item presented in position $X$ for the two conditions is as follows:

Inclusion: $I O_{R}$

Exclusion: $I\left(1-O_{R}\right)$

Given this, a relatively pure assessment of item memory and of order memory can be obtained through simple algebra. Item memory can be obtained by summing 
performance on the inclusion and exclusion conditions, and order memory can be ascertained by dividing the inclusion condition by $I$.

Given that a number of tasks have been developed to separate item and order memory, it is surprising that few studies exist that examine the relative influence of long-term memory effects on item and order memory. The research that does exist is reviewed below.

\subsection{Lexico-Semantic Effects on Memory for Order}

As lexico-semantic effects have typically been assessed using a correct-inposition criterion in an ISR task, they could reflect an influence on item memory, on order memory, or on both. In recent years there has been a move toward determining which of these processes is affected by long-term memory. To do this, researchers have re-examined effects of word frequency, language familiarity, lexicality, and imageability, using tasks that dissociate memory for item and memory for order.

\subsubsection{The Lexicality Effect on Memory for Order}

Saint-Aubin and Poirier (2000) found a typical lexicality effect on the ISR of words and non-words under quiet and articulatory suppression conditions. With regards to memory for order, Saint-Aubin and Poirier found a significant effect of lexicality on conditional probabilities, with fewer order errors for non-words than for words. There were also more order errors in the suppression condition than under quiet conditions. The interaction between articulatory suppression and lexicality was inconclusive as there was an insufficient volume of order errors for non-words.

While Saint-Aubin and Poirier (2000) found fewer order errors for non-words, other studies have failed to reveal a robust influence of this variable on memory for order. Gathercole and her colleagues (2001) examined the lexicality effect in serial recall and serial recognition using lists that varied in length between 2- and 6-items. Gathercole et al. (2001) found the typical lexicality effect in serial recall, with words remembered better than non-words. An overall lexicality effect was not observed for serial recognition, although there was an interaction between list length and lexicality. Further analyses revealed a significant lexicality effect for 6-item lists only. In a 
separate experiment, Gathercole et al. (2001) examined serial recognition performance in adults, finding a significant effect of lexicality with better performance for words over non-words. This effect was of a small magnitude when compared to the finding from serial recall. Despite finding significant effects of lexicality on serial recognition, Gathercole and her colleagues maintain that lexicality exerts its influence on item memory but not on order memory. They note that the difference in magnitude of the effect size in the two tasks indicates that the variable exerts more of an influence on item memory. In addition, the researchers suggested that the significant lexicality effect for longer lists could be attributed to a decline in reliance on phonological information for lengthy lists coinciding with an increased reliance on semantic associations between items at this list length.

\subsubsection{The Word Frequency Effect on Memory for Order}

The influence of word frequency on order information has typically been assessed using a conditional probability analysis in a serial recall task. Poirier and Saint-Aubin (1996) presented participants with lists of high, medium and low frequency words. They found the typical word frequency effect on item information, but no influence of word frequency on conditional probabilities. They concluded that word frequency exerts its influence on item memory but not on order memory.

Stuart and Hulme (2000) replicated this finding in an ISR task, examining pure and mixed lists of high and low frequency words with or without a familiarisation procedure. They found a frequency effect on the correct-in-position analysis, but no effect of word frequency on conditional probability scores. There was also no effect of the familiarisation procedure on the proportion of order errors, suggesting that word frequency does not influence memory for order.

Allen and Hulme (2006) examined ISR performance on word sets that differed according to word frequency and phonological neighbourhood size. Typical facilitative effects were found for both variables, and while there was an effect of phonological neighbourhood size on the conditional probabilities, no such effect was found for the word frequency sets. The finding from Allen and Hulme (2006) is consistent with previous research in suggesting that word frequency does not influence memory for order. 
One study did show a small but significant effect of word frequency on a conditional probability analysis of serial recall data. Hulme, Stuart, Brown, and Morin (2003) investigated the serial recall of high and low frequency words in pure and mixed lists. They found fewer order errors for high frequency words compared with low frequency words but no difference in the overall proportion of order errors between pure and mixed lists. This differs from previous findings of no frequency effect on order memory.

\subsubsection{The Language Familiarity Effect on Memory for Order}

Thorn et al. (2002) examined serial recognition for order performance on French/English bilinguals with either French or English as their native language. As discussed previously, participants showed a language familiarity effect in ISR with better performance for the native language over the second language. This had been interpreted as an instantiation of the word frequency effect and the phonotactic probability effect. That is, word forms are encountered more often in the native language than in the second language and this was assumed to support the recall of the native language for both words and non-words using redintegration processes. Participants showed no native language advantage for the serial recognition of order task, indicating that this variable did not affect memory for order.

\subsubsection{The Concreteness Effect on Memory for Order}

The findings relating to concreteness and imageability effects on memory for order are contradictory. Walker and Hulme (1999) examined the effect of concreteness on short-term memory using a serial recall task and a matching span task. Despite the clear advantage for concrete over abstract words in serial recall, Walker and Hulme failed to find an effect of this variable on matching span. In addition, no significant effect of concreteness was found on the proportion of order errors in serial recall. These results were interpreted as a long-term memory effect operating at output. More recently, Miller and Roodenrys (2009) found no influence of concreteness on memory for order using a conditional probability analysis of ISR data. 
Some studies have observed effects of concreteness on memory for order. Allen and Hulme (2006), for instance, found a significant effect of concreteness on conditionalised order errors. In particular, abstract words had a higher proportion of order errors than concrete words, indicating that memory for order was more stable for items where participants could generate a concrete image of the word.

Another semantic variable, word pleasantness, has been shown to influence memory for order. Monnier and Syssau (2008) examined serial recognition performance on pleasant words and neutral words. Pleasant words were defined as words that evoked a pleasant feeling (i.e., gift). They found that serial recognition accuracy was greater for pleasant words than for neutral words.

\subsection{Adapting the Redintegration Hypothesis: The Retrieval-Based Hypothesis}

Research to date has shown that long-term memory variables have dissociable effects on memory for order and memory for items. Word frequency and language familiarity have been shown in most instances to influence item memory but not order memory. The influence of other variables, such as lexicality and concreteness, on memory for order is less clear. Some studies have shown inhibitory effects of concreteness and lexical status on memory for order, while others have failed to find an effect. The redintegration hypothesis is unclear whether a long-term memory effect on order should be anticipated, as it factors item and order information into a single score. To rectify this, Poirier and Saint-Aubin (1996, also see Saint-Aubin \& Poirier, 1999a, 1999b) adapted the reconstruction hypothesis to include two additional assumptions regarding the influence of long-term memory on short-term memory. These are (a) that phonological representations are output in the appropriate order and (b) order errors are attributed to problems in the reconstruction of degraded phonological traces (Saint-Aubin \& Poirier, 2000). They distinguished these additional assumptions from the redintegration hypothesis by calling their approach the retrieval-based hypothesis.

According to the retrieval-based hypothesis, a phonological representation of the items is created when the list is presented. These phonological representations are encoded in order of appearance, and the representations are subject to degradation. At 
recall, items are retrieved in the appropriate order and the degraded phonological trace serves as a retrieval cue for the appropriate long-term memory representation. Item recall is influenced by the efficacy of the retrieval process and is thus influenced by factors that increase the accessibility of an item in long-term memory, such as word frequency. Item recall is also hindered by factors that influence degradation of the phonological cues such as articulatory suppression.

In the retrieval-based hypothesis, order errors occur due to trace reconstruction problems during the recall process. That is, if certain phonological features are common to a number of items in the list, then an item that has lost any distinguishing featural information may be erroneously interpreted as one of the other list items.

The retrieval-based hypothesis predicts better order memory for non-words than for words, as non-words by-pass the redintegration process. Importantly, the retrievalbased hypothesis predicts no effect of long-term memory variables on memory for order as connections with long-term memory occur only after items have been retrieved in their order. Only variables that influence the phonological uniqueness of the list items will influence memory for order. Thus the retrieval-based hypothesis accurately predicts no influence of language familiarity or word frequency on memory for order. It also predicts no effect of concreteness on memory for order, although this variable has been shown to exert a small influence in some experiments. Crucially for the current thesis, the retrieval-based hypothesis predicts no effect of phonological neighbourhood density on memory for order, as neighbours do not appear within the trial lists in neighbourhood density experiments.

\subsection{The Phonological Neighbourhood Effect on Short-Term Memory for Order}

While the phonological neighbourhood's influence on performance in a serial recall task is well established, little attention has been paid to this variable's influence on order memory. In fact, only a small part of Roodenrys et al. (2002) and Allen and Hulme (2006) papers were devoted to reporting order effects, and neither paper discussed the findings in length. Roodenrys et al. (2002) investigated the effects of phonological neighbourhood density by varying neighbourhood size, along with other variables, within lists and used regression to analyse the data. Roodenrys and his 
colleagues found that large phonological neighbourhood words were more likely to be recalled out of their presented position than small neighbourhood words.

Allen and Hulme (2006) examined the effect of phonological neighbourhood density on serial recall. Using pure lists, they found that lists of words from large phonological neighbourhoods were more likely to be recalled in the correct serial order than were lists of small phonological neighbourhood words. Examining conditional probabilities, they found a smaller proportion of order errors for large than for small neighbourhood sets ( .25 for large neighbourhood words and .29 for small neighbourhood words). Despite the small magnitude of the order errors, the difference between the large and small neighbourhood sets was significant.

\subsection{Explaining the Phonological Neighbourhood Effect on Memory for Order: Inadequacies of the Retrieval-Based Hypothesis}

According to the retrieval-based hypothesis, long-term memory exerts its influence at the point of recall after the order of output had been determined. Accordingly, long-term memory effects such as word frequency and phonological neighbourhood size should not influence memory for order. This hypothesis dictates that order errors occur during redintegration only if the phonological information relating to a trace has decayed to a point where it is indistinguishable from other list items. As phonological neighbourhood density is thought to influence the reconstruction of a trace rather than the degradation of the items it should not influence memory for order. This is in clear contrast to findings from both Roodenrys et al. (2002) and Allen and Hulme (2006). Further, the pure list result cannot be explained as an encoding advantage for large neighbourhood words, as perception of large neighbourhood words is more cumbersome than perception of small neighbourhood words (Luce \& Pisoni, 1998).

Roodenrys et al. (2002) suggested that long-term memory might influence an order maintenance mechanism. Using the principles of competitive queuing (Houghton, 1990), Roodenrys and his colleagues proposed a queue-jumping explanation of neighbourhood effects in mixed lists. According to competitive queuing, items are activated during presentation with the proportion of allocated activation decreasing across serial position. At recall, the most active item, 
presumably the first item, is selected for recall and its activation is subsequently suppressed. Recall then proceeds by selecting the next most active item and so on. The queue-jumping hypothesis suggests that if long-term memory is able to contribute to activation at this stage, then words from large phonological neighbourhoods may have a greater activation as a result of facilitative links with other similar sounding words. This additional activation may result in the word being recalled ahead of its position, displacing words from smaller phonological neighbourhoods that do not have the benefit of such high facilitative activation. This hypothesis would necessitate a link between long-term memory and short-term memory earlier than that suggested by the retrieval-based hypothesis.

For the queue-jumping hypothesis to be supported, large and small phonological neighbourhood words must remain stable in pure lists, as the additional activation given by neighbours should be equal among list items. Contrary to this, Allen and Hulme (2006) reported effects of neighbourhood density on memory for order using pure lists of large and small neighbourhood words. More specifically, they found that after controlling for the differences in item recall, large neighbourhood words were more likely to be recalled in their correct serial position than lists of small phonological neighbourhood words. While the result challenges the explanation offered by Roodenrys and his colleagues, the two findings at least suggest that long-term memory does influence short-term memory prior to recall.

Apart from the contradictions raised by the Allen and Hulme finding, the queue-jumping hypothesis remains untested. Further, no research has been conducted to replicate the finding in mixed lists or that found in pure lists, and no attempt has been made to address these findings within models of short-term memory.

\subsection{Conclusion}

Evidence from Roodenrys et al. (2002) and Allen and Hulme (2006) suggested that phonological neighbourhood density has an effect on memory for order. This effect has not, as yet, received a satisfactory explanation, and is in contrast to predictions made by the redintegration hypothesis and the retrieval-based hypothesis. Examinations of other lexico-semantic variables have not shown reliable influences on memory for order, keeping with predictions made by the redintegration hypothesis. 
The question of whether long-term memory influences short-term memory for order is important when trying to elucidate exactly how short-term memory and longterm memory interact. The redintegration hypothesis and the retrieval-based hypothesis both posit a long-term memory effect occurring only at the point of recall. This is incompatible with the finding of a phonological neighbourhood effect on short-term memory for order, which suggests an earlier interface with long-term memory.

The finding also has implications for the argument that item and order memory subsume separate processes. The finding that long-term memory variables such as word frequency exert an influence on item memory but not on order memory serves as further evidence for separate processes. If the phonological neighbourhood effect does influence memory for order, and if the finding of no effect on memory for order for the other lexical variables is due to the inadequacies of the serial recall task, then an argument could be put forward for a common process. 


\section{Chapter Six}

\section{Models of Memory for Order}

\subsection{An Overview of Models}

Since James (1890) initially ascribed structure to memory, a plethora of cognitive memory models have been created ranging from simple box schematics, to algorithmic models, with a more recent focus on computational models. Despite being influential with regards to conceptual development, box-and-arrow models (i.e., Baddeley \& Hitch, 1974) fail to give a detailed account of the mechanisms underlying memory, and in particular the mechanisms underlying memory for order. For instance, while Baddeley (2000) attributes the influence of long-term memory on item information to the link between the phonological loop and language (see Figure 3.1), and memory for order to the link between the episodic buffer and episodic long-term memory (Burgess \& Hitch, 2005), the exact mechanisms involved in the maintenance and retrieval of these two types of memory remains unspecified. Contemporary models of memory for order provide a more quantitative account of the process of short-term memory, giving explicit detail to the mechanisms underlying the system.

Models of order can be classified in a number of ways, but primarily diverge on whether order is stored using associative or non-associative mechanisms (Hitch, Fastame, \& Flude, 2005). Models that represent order via item-to-item associations or via item-to-position associations are regarded as associative models, while models that store order without reference to other list items or an external order mechanism are called non-associative models. Within this initial dichotomy, models may fall under a number of sub classifications depending on the type of mechanism used to store order and the way positions in a sequence are defined. Models can be defined by how position is coded. Position can be coded using temporal, absolute or relative terms (Henson, 1999). Temporal coding involves coding items based on their time of presentation relative to the start or end of a sequence. Absolute coding assumes that items are associated with their ordinal position (first, second, third) regardless of timing of presentation. A coding of relative position assumes that items are coded according to their position relative to the start and end of a list. 
Models can be also categorised as to whether order is stored using chaining, positional or ordinal representations (Henson, 1999). Chaining models rely on itemto-item associations to recall items, making the approach an early instantiation of an associative model (Marshuetz, 2005). For instance in the series $A, B, C, D$, order may be given by pair-wise associations between $A$ and $B, B$ and $C$, and $C$ and $D$, as in the case of simple chaining, or may be given by compound chaining mechanisms that include associations with non-adjacent items. Early chaining models have since evolved into elaborate computational models such as the Theory of Distributed Associative Memory (TODAM: Lewandowsky \& Murdock, 1989).

Positional models are regarded as associative models because items are associated with their sequential position. For instance, in the list $A, B, C, D$, order may be coded in an absolute way by associating each letter with its position, $A 1, B 2, C 3$, $D 4$,or in a relative way by associating each letter with its relative position from the start or end of a sequence, i.e. $C$ is the $3^{\text {rd }}$ item from the start. Positional models have enjoyed popularity in recent years, with the development of phonological loop model (Burgess \& Hitch, 1992, 1999), the OSCAR model (Brown et al., 2000), and the startend model (Henson, 1998a). Positional models differ from chaining models in that the retrieval cue for the former is a positional code, while the retrieval cue for the latter is the previous item (Henson, 1998a).

Ordinal models store activation through the relative strength of items. For instance, in the sequence $A, B, C, D$, order is represented by a gradient of activation, with the amount of activation allocated to each item decreasing exponentially after the first item. Ordinal models differ from chaining and positional models in that order is represented without reference to other list items (Henson, 1998a). This makes ordinal models, such as the Primacy Model (Page \& Norris, 1998), instances of nonassociative models.

All of the above models posit a memory system that is distinct from other cognitive functions. There is another group of models that ascribe short-term memory for order to other cognitive systems. For instance, there is a subset of models that describe how serially ordered behaviour could occur within a language system, called psycholinguistic models, and a subset of models that describe how memory for order could occur within an attention system. The aim of this chapter is not to provide a comprehensive account of various models but to outline approaches to memory for 
order with an emphasis on predictions made about an influence of long-term memory on short-term memory for order.

\subsection{Chaining Models}

Chaining is one of the earliest mechanisms of serial order, being first proposed by Ebbinghaus (1913) as a model for serial learning. Chaining follows from stimulusresponse theory where each response becomes a stimulus for the next (Henson \& Burgess, 1997). The simplest type of chaining model involves pair-wise associations between adjacent items, with each item acting as a cue for the next during recall.

A number of fallibilities of chaining models have been identified, undermining the viability of this type of mechanism of memory for order. First, as the previous response must be generated to cue the next response, simple chaining models are unable to account for error patterns observed in normal performance. For instance, chaining is unable to explain how recall recovers from error and how repeated items in presentation are able to serve as a cue for more than one item (c.f. Murdock, 1993; Wickelgren, 1966). Simple chaining predicts that if an error occurs on an item, then that item will not be an effective cue for the next item, leading to a failure to recall all subsequent items. Recall performance shows that this is not the case. In addition, it is unclear how, in the sequence $A, B, A, C$, that item $A$ can act as a cue for both $B$ and $C$. Chaining is further criticised for its assumption that item and order information are stored in a unitary manner. Order errors, according to such models, are due to a loss of item information rather than a loss of order information (Healy, 1974). As such, chaining has difficulty explaining differential effects on order memory and item memory, and does not provide a testable account of how the bins or slots are searched in the correct order (Brown et al., 2000). Lashley (1951) further proposed that simple chaining could not account for the speed of serial acts, and that a chaining explanation was inadequate for accounting for the complexities of language production. Finally, Burgess and Hitch (1992) examined serial order data and concluded that models with little or no chaining came closest to replicating empirical results.

In response to this, chaining models evolved from using simple associations into compound chaining models, where each item is connected with all or many other items (Murdock, 1995). Such models are better equipped to handle repeats in recall, 
and are not as devastated by an error as simple chaining models. A schematic interpretation of chaining models is shown in Figure 6.1.

\section{Storage}

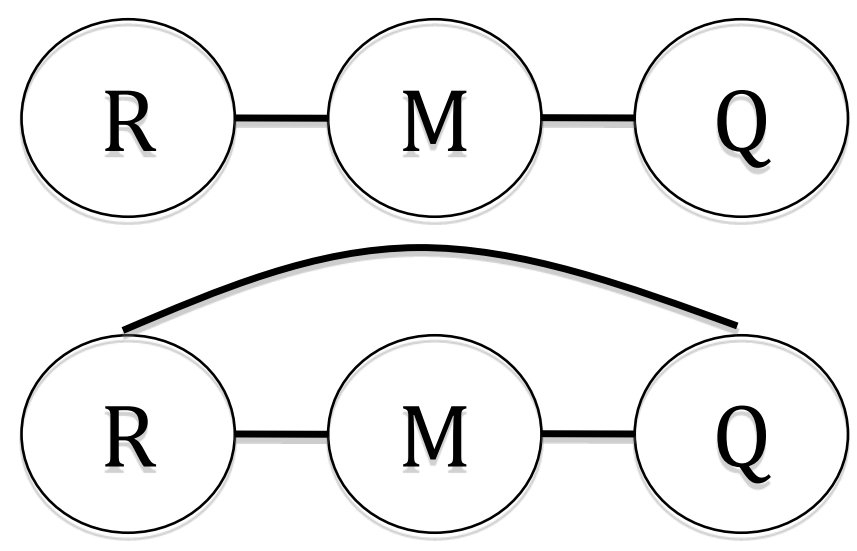

Retrieval

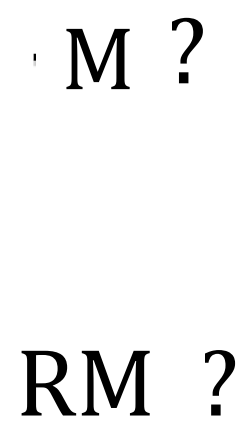

Figure 6.1 The upper diagram represents simple chaining models, while the lower represents compound chaining models. Replicated with permission from Henson's (1996) unpublished $\mathrm{PhD}$ thesis.

Juxtaposed against the vast body of evidence accumulated against chaining models is a smaller body of evidence in support of chaining. Serra and Nairne (2000) investigated the role of item-to-item associations in memory for serial order using a part-set cuing task. In this task participants performed serial reconstruction on eightitem lists. Participants were required to reconstruct four of the eight items. In the uncued condition, the four target items were indicated and no other positional information was given. In the cued condition, the four non-target items were placed in their serial position and participants were required to reconstruct the order of the target items based on this additional information. A strictly positional account would predict that performance on the cued condition would not differ from the uncued condition as items are coded without reference to other list items. Alternatively, itemto-item associative models would predict that performance would be better in the cued condition than in the uncued condition. Results supported the latter and Serra and Nairne concluded that while item-to-item associations may not be the only 
mechanism of serial order, these associations can be used to remember the order of sequences.

Despite the evidence weighted in favour of models that do not rely on item-toitem associations, chaining models continue to be proposed as valid memory models. For instance, TODAM (Murdock, 1982; TODAM2: Murdock, 1993) stores order via pair-wise associations between successive items. In TODAM, lists of items are contained within a memory vector that holds both item and associative information. Input and output in TODAM involves the processes of convolution and correlation respectively. Convolution is a means of combining information, while correlation "unpacks" information (Murdock, 1997). Item information is stored in a perceptual (P) system by adding item vectors to a memory vector. Order information is coded by convolving item vectors with neighbouring item vectors and the convolution is added to the memory vector, stored within a query (Q) system (Murdock, 1982). The response (R) system is responsible for recall. Recall is achieved by correlation, where each item acts as the cue for the next item in a sequence. This results in an approximation of the item vector for that item. For instance, in the sequence $A, B, C$, the item $A$ will cue item $B$ resulting in the retrieval of $B$ '. The item vector is then 'deblurred' to produce a response. This involves competition between the item $B$ and its two neighbouring items $A$ and $C$. The item that is closest to $B$ ' will be produced as a response. This may lead to order errors during response when an approximation of an item vector, $B$ ', might be a closer approximation to item $A$ than it is to item $B$, leading to the recall of $A$ in the place of $B$.

The processes of correlation and convolution allow TODAM to overcome many of the shortcomings encountered by chaining models. For instance, TODAM is able to account for recovery from error because the result of correlation is a blurry vector that can act as a cue for the successive item even if the item itself cannot be ascertained from the vector.

The influence of long-term memory on short-term memory is addressed by modelling word frequency effects. According to an updated version of the model, TODAM2 (Lewandowsky \& Murdock, 1989), word frequency influences the convolution process, such that the strength of encoding for low frequency words will be less than that of high frequency words. This involves the influence of a long-term memory process at encoding, rather than at retrieval. It is unlikely that the phonological neighbourhood effects evident in memory tasks occur during encoding, 
as large neighbourhood words are recognised more slowly than their small neighbourhood counterparts (Luce \& Pisoni, 1989). However, if neighbourhood size was able to influence the strength of the encoding process, then TODAM may be able to model the Roodenrys et al. (2002) finding. If large neighbourhood words have a stronger activation during convolution then it is possible that, during correlation, the vector for a small neighbourhood word might be a better approximation of its larger list neighbour than it is of the small neighbourhood target word.

\subsection{Positional Models}

The plethora of criticisms levelled against chaining contributed to a rise in the popularity of positional models. Positional models are, like chaining models, associative in nature; however they avoid problems faced by chaining models by assuming that instead of being associated with one another, items are associated with a positional cue such as a context signal. Conrad's (1965) slot model of short-term memory is an early example of a positional model. According to Conrad's model, items are stored in a series of boxes, and recall proceeds by stepping through the boxes in their serial order. This type of model overcomes the problems faced by chaining because recall can continue even if an error is encountered with an earlier item. Further, repeated items do not present a problem for the model because items are stored in separate boxes. Evidence for positional models comes from error patterns observed in ISR. Conrad (1960) found that if an item from a previously presented list intrudes on the current list it tends to maintain its same serial position. Further to this, Henson (1999) found that if the final item in a previous list intrudes on the current list, it tends to maintain its relative position as the final item, regardless of whether lists are different lengths.

In addition, positional models of memory began to emerge in the literature due to the success of positional theories in the area of speech production. Namely, the success of Houghton's (1990) competitive queuing model in explaining word pronunciation from long-term memory spurred the rise in using a positional explanation of serial order in short-term memory. Competitive queuing consists of three primary elements. The first element is a set of nodes that represent items to be composed into a list. Second, these item nodes are activated in parallel via weighted 
connections to a temporally mediated "control signal". Third, items are subject to a competitive filter, which selects and outputs the most active node at each time step during production. This basic architecture has been adapted by theorists to account for short-term memory for order, most notably by Burgess and Hitch (1999) in their network model of the phonological loop. The phonological loop model mimics that of Houghton (1990), with position in both models coded relative to the start of the sequence. However, the phonological loop model employs a more complex multinode timing signal than that used by Houghton (Glasspool, 1995).

Other positional models also employ a competitive queuing mechanism but differ in the implementation of this signal. The oscillator-based associative recall (OSCAR) model (Brown et al., 2000) uses a context-timing signal to code items relative to the start of the list coupled with a competitive queuing mechanism. In OSCAR, items are coded by creating an item vector that is associated with a learning context vector comprised of the signals of a set of oscillators. The set includes oscillators of different frequencies so that the overall vector (learning context) changes in a manner that produces distinct patterns across time. At recall, the learning context is reinstated and is used to cue the item vector. The cued item vector then undergoes a redintegration process, where it is compared to a response vector containing all of the list items and some items that were not in the list. The most active item at this point will be output using a competitive queuing mechanism. Brown et al. (2000) note that order errors occur during this second stage, depending on the quality of the dynamic retrieval cue and the extent that a cue is specific to an item in question.

One further positional model that also employs a competitive queuing mechanism, but varies from other positional models with regards to the nature of the context-timing signal is the start-end model (SEM; Henson, 1998a). SEM employs two markers that are situated at either end of a list. These markers mean that items are coded relative to both the start and to the end of a sequence. The presence of these oscillators is evidenced by the finding that items will tend to intrude on the same position relative to the start and the end of the sequence in lists where length varies (Henson, 1999). SEM further differs from the account offered by Houghton (1990) and Burgess and Hitch (1999) in that it codes items using relative coding while the latter theories use a temporal coding mechanism. According to SEM, items are stored as position-sensitive tokens. These tokens contain a number of components, some 
representing item information and some components representing order information. Items are unordered while they are stored in short-term memory. Ordering occurs during recall, and is determined by reinstating the positional code of the position to be recalled. The cue is matched against all items, and activates each token representation to the extent that it matches the positional cue. Items with similar positional cues, that is, neighbouring items, will be activated in proportion to their similarity to the presented cue. The strongest item will be selected for output using a competitive queuing mechanism. Interestingly, unlike previous models, SEM does not include a redintegration process. Instead, long-term memory effects are explained as variations within the short-term memory system. According to Henson (1998a), the frequency effect on item information may be due to a greater base-line level of activation of the phonological or categorical token representation of high frequency items. In addition, the lexicality effect is attributed to differential encoding for words and non-words, with words coded as chunks, while non-words are stored using their individual phonemes. If the phonological neighbourhood effect can also be attributed to higher baseline activation for the item-specific token representation of large phonological neighbourhood words, then it is possible that large neighbourhood words may be recalled ahead of neighbouring small neighbourhood words. Note that this would mean that word frequency should show similar effects in mixed lists; this has not been found in the literature (see, for instance, Stuart \& Hulme, 2000).

\subsection{Ordinal Models}

Ordinal models, unlike chaining and positional models, do not require associations to represent the order of a sequence. Instead, order is stored via a gradient of activation, a concept that was first introduced by Grossberg (1978). According to ordinal models, the amount of activation given to a particular item is a function of the level of activation given to previous items. Grossberg (1978), for instance, theorised that more recently presented items will have a higher level of activation in short-term memory, giving an increasing gradient of activation.

Early ordinal models included the perturbation model of Estes (1972) and Lee and Estes $(1977,1981)$. The perturbation model provided an account of memory where order is stored according to its relative position in a list. During presentation and any subsequent retention period there is a reactivation of the associative links 
between list items during which there is a probability that items may perturb. The perturbation process systematically increases the uncertainty about the position of an item in a list. Despite its age, the perturbation model remains a valid way of representing serial order, and was recently adopted by Neath (1999) for the serial order component of an adaptation of the feature model (Nairne, 1990; Neath \& Nairne, 1995).

More recently, Page and Norris's (1998) Primacy Model gained prominence as a model of memory that represents order using an ordinal mechanism. The Primacy Model models order using a gradient of activation similar to the concept introduced by Grossberg (1978), but differs in that it assumes a decreasing gradient of activation. That is, the order of items $A, B, C, D$ can be given by the pattern of activation such that $\mathrm{x}_{\mathrm{A}}>\mathrm{x}_{\mathrm{B}}>\mathrm{x}_{\mathrm{C}}>\mathrm{x}_{\mathrm{D}}>0$. This concept is outlined in Figure 6.2.

\section{Storage}

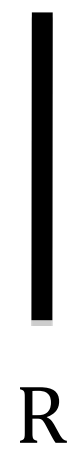

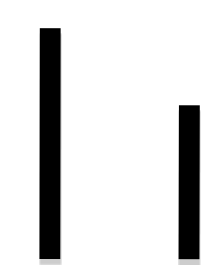

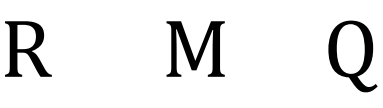

\section{Retrieval}

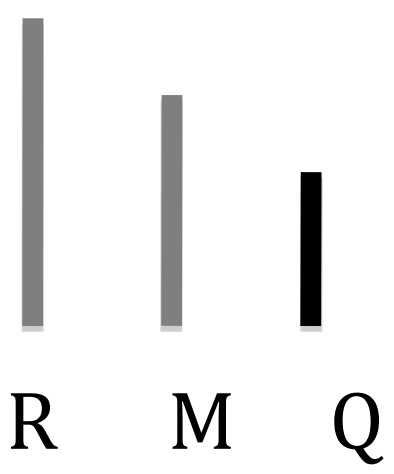

Figure 6.2 Representation of serial order in an ordinal model, replicated with permission from Henson's (1996) unpublished PhD dissertation. The lighter lines represent suppression, while the darker lines represent activation.

The model assumes localist representation with the amount of activation given to an item node increasing for a short period after the item is presented and then decaying exponentially. Degraded representations are retrieved from this stage in order using a competitive queuing mechanism. Order errors may occur during this process because of noise in the gradient of activation. The retrieved item trace is then forwarded to a second stage where any information that has been lost from the trace can be reinstated by matching the output to an appropriate localist node. This process activates not only the node that matches the trace, but also partially activates other nodes to the extent that they are phonologically similar to the item. The node that 
corresponds to the selected item will have an activation of 1 . Nodes that are phonologically similar to the item will have an activation of $S$, where $0<S<1$.

Distinct items will have an activation level at this stage of 0 (Page \& Norris, 1998). As items are retrieved in order prior to the influence of phonologically similar items, the Primacy Model would predict no influence of long-term memory on short-term memory for order.

\subsection{Memory for Order as part of the Language System}

While the above models differ with regards to the mechanisms used to maintain items in their order, they are all similar in that this process is done within a distinct memory system. An alternative body of models, called psycholinguistic models, place verbal memory within the language system. Psycholinguistic models have some intuitive appeal given parallels between the requirements for performing a short-term memory task, and those involved in language processing. For instance, in order to perform any short-term memory task, one must have the capacity to perceive words and to produce words. Similarly in language processing, memory for order is paramount as both processing and producing an utterance involves remembering the order of words in a sentence, and phonemes within a word (Acheson \& MacDonald, 2009a).

In addition to parallels in the processes required for both linguistic processes and short-term memory for order, there are also comparisons between the two processes in terms of output. Ellis (1980) noted that errors occurring in a working memory task mimic those occurring naturally in speech production. Fromkin (1971) noticed that errors in speech are subject to similar constraints of distance, as errors in serial recall. Further, Shattuck-Hufnagel (1979) found that speech errors are more common under conditions of phonological similarity, much like the finding in serial recall. In addition, like recall errors, speech errors are lexically restrained, meaning that an error in speech is more likely to result in a word than in a non-word (Baars et al., 1975). Evidence of parallels between memory and language does not necessitate a common underlying system, but does enhance the viability of such an approach (Acheson \& Macdonald, 2009b). This given, it is perhaps surprising that the first substantive effort to create a unitary account of production and memory was made less than two decades ago. 
Hartley and Houghton (1996) first attempted to integrate speech production and short-term memory to account for new word learning. Hartley and Houghton's model combined speech production architecture with the serial order mechanism implemented in Burgess and Hitch's (1992) phonological loop model. Hartley and Houghton's model was criticised for its proposal of a common input and output buffer, based on the finding of differential effects of phonological neighbourhood density on speech perception and speech production (Allen \& Hulme, 2006).

Other psycholinguistic models, such as that created by Martin and Saffran (1997) provide a better fit for such results, owing to the inclusion of separate input and output buffers. Martin and Saffran's (1997) interactive activation explanation of speech production and working memory addresses memory as a pattern of activity across localist nodes. The model has its genesis in the Dell and O'Seaghdha (1992) interactive activation model of production. The model assumes modular representation, that is, there are separate semantic, phonological, lexical and morphological levels of representation. Bidirectional connections link levels of representation, allowing feed forward and feedback activation. Within each level, information is represented using localist nodes. Activation to each of the levels (semantic, phonological, lexical and morphological) is serial, with phonological representations activated first, and activation is then passed to and from the other layers via the bidirectional connections until a response is required. As the phonological nodes are activated first they will be the strongest early representation of the word. Over the time course of the process, semantic features will begin to receive activation and they will have more influence on word selection. However, the phonological features will continue to have more of an influence than semantic features as these receive continuous feedback throughout the process (Martin \& Saffran, 1997).

While production based models have had great success in explaining singleword utterances, most would need to be extended to explain the serial order of multiword utterances. Some models that account for production of multiword utterances constrain word order using rule-governed frames (Dell, Burger, \& Svec, 1997; Dell, 1986). Serial order is still modelled as recurrent connectivity between the phonological and lexical-semantic level, which is constrained by imposing syntactic rules on the sequence (e.g., Determiner-Adjective-Noun; Acheson \& Macdonald, 2009a). While such a framing system may be helpful in explaining sentence 
production, it does not account for findings in serial recall where lists have no discernable syntax. Such models, therefore, would need to be extended to account for ISR performance.

Martin and Saffran (1997) note that the Dell and O'Seaghdha (1992) model could be extended to account for serial order in short-term memory using the same basic assumptions. Items early in the list receive more facilitative feedback from the entire system than items late in the list. This means that items early in the list will have more support from all layers of representation including semantic representation, while items later in the list will primarily be maintained by their phonological representations. The basic principles of competitive queuing apply, with the most active item selected for output first and then suppressed. As the first item will receive the most feedback activation from within the system it is likely to be the most active item. Decay is also inherent within the system, and the primacy effect is explained as the items at the terminal serial positions being less effected by decay than earlier items. Order is maintained via connections within the lexical system and a sequence place-holder that is established during presentation (Martin \& Saffran, 1997).

Gupta and MacWhinney (1997) proposed a model to account for the relationship between word learning and verbal short-term memory. This is a multitiered model with separate input and output buffers, each joined to separate phoneme layers. Again, separate input and output buffers allow the model to account for separate effects of phonological neighbourhood density on speech perception and speech production. The phoneme layers are connected to a phonological chunk layer, containing word forms, which are topologically organised with phonological neighbours residing close together with similar representations. The phonological layer and the chunk layer are both competitive queuing structures with a set of nodes and an associated filter. The model includes two processes that govern memory for order. The first is a phonological store, which is an automatic process that is similar to the temporal ordering mechanism used in the Burgess and Hitch (1992) model. The second is a context maintenance system, which stores a series of vectors that represent a pattern of activation over the chunk layer at a single point in time. This is a controlled and strategic process. The model also includes a semantic layer, connected to the chunk layer, and a speech output planning system, containing mechanisms required for the motor programming of speech. 
While psycholinguistic models continue to evolve into the short-term memory sphere, little work has been done on using these models to explain long-term memory effects on short-term memory for order. In the Gupta and MacWhinney (1997) model, lexicality is attributed to fewer resources available for strategic chunking for nonwords as additional rehearsal is required for creating a node for non-words in the chunk layer. They do not attempt to explicate the mechanisms behind other long-term memory variables. Alternatively, Acheson and Macdonald (2009a) suggested that people use their implicit knowledge of sounds to constrain the order in which the sounds are produced. Large neighbourhood words contain common sound sequences and this helps to constrain the order of phonemes in these words. This explanation is proposed for the neighbourhood effect on memory for items and does not address memory for order. While Acheson and MacDonald's explanation is a step toward addressing long-term memory effects within a psycholinguistic model, the mechanisms involved need to be explicated before any predictions regarding a neighbourhood effect on memory for order can be made.

\subsection{Conclusion}

Despite decades of theoretical development, no clear consensus on the architecture of short-term memory has been reached. While the perennial debate centres on the nature of the item and order representations, no current instantiation of order memory can account for an influence of neighbourhood size on memory for order. However, before a revision of models of memory for order can be called for, the nature of the phonological neighbourhood effect on memory for order must be identified. The following chapters compose the empirical portion of this thesis, with the aim to explicate the effect this variable has on memory for order. 


\section{Chapter Seven}

\section{Investigating the Phonological Neighbourhood Effect on Short- Term Memory for Order}

\subsection{Introduction}

Research suggests that phonological neighbourhood density not only influences the probability of correct item recall, but it also influences the probability that an item will be recalled in its correct serial position (Roodenrys et al., 2002; Allen \& Hulme, 2006). This effect has been reported in trials where neighbourhood density varies within lists, known as mixed lists, and in trials where neighbourhood density is consistent within lists, known as pure lists. In mixed lists, words from large phonological neighbourhoods were more likely to be recalled in a different serial position with the suggestion that large neighbourhood words displace small neighbourhood words to 'jump the queue' in recall (Roodenrys et al., 2002). In pure lists, large neighbourhood words were more likely than small neighbourhood words to retain their serial position (Allen \& Hulme, 2006).

The effect of neighbourhood density on item memory is typically attributed to redintegration processes operating at the point of recall (Roodenrys et al., 2002). However, the finding of an effect on memory for order is novel, and is difficult to reconcile with many contemporary models of short-term memory. Specifically, the result is inconsistent with predictions made by two-stage models, where the first stage, which involves the maintenance and retrieval of items in their order, is independent of long-term memory (Page \& Norris, 1998; Burgess \& Hitch, 2006). According to such models, the long-term memory influence only occurs during the second stage, after the order of items has been retrieved. As such, variables such as phonological neighbourhood size and word frequency will only influence the probability of correctly deblurring or reconstructing the phonological trace, and will not influence memory for order.

The finding that phonological neighbourhood density does influence memory for order, therefore, has important implications for models of memory for order, and 
will help to identify the locus of the long-term memory influence on short-term memory processes. The aim of the first stage of experiments is to replicate the findings from Roodenrys et al. (2002) and Allen and Hulme (2006) using mixed and pure lists in tasks thought to separate item and order memory. Ethics approval was granted from the Human Research Ethics Committee at the University of Wollongong (ethics approval number HE06/11).

\subsection{Experiment 1: Investigating the Neighbourhood Effect in Serial Recall using Mixed Lists}

\subsubsection{Rationale}

Given the effect identified by Roodenrys and his colleagues is novel and has important conceptual and theoretical implications, it is necessary to replicate the effect and identify the conditions under which it appears. Roodenrys et al. (2002) proposed a queue-jumping hypothesis to explain their result. According to this hypothesis, a word's phonological neighbourhood is able to contribute to its activation level during maintenance. Words from large phonological neighbourhoods would receive more of this facilitative activation than would small neighbourhood words, on account of their greater number of neighbours. If it is assumed that the order in which items are selected is based on their relative activation level using a competitive queuing mechanism, then it is feasible that words from large phonological neighbourhoods may be recalled ahead of their serial position if by doing so they displace a small neighbourhood word.

The aim of Experiment 1 was to replicate the neighbourhood effect found by Roodenrys et al. (2002), by examining the effect of phonological neighbourhood density on ISR performance using mixed lists of large and small neighbourhood words, while teasing apart the effects of this variable on item and order information. , Rather than employing the regression design used by Roodenrys and his colleagues, stimuli were arranged so that large and small words alternated within lists. This was done to maximise the differences in neighbourhood size between the items in the list. To assess item memory, recall data were analysed using a correct-in-position criterion and a free recall criterion. As outlined in Chapter 5, using the total number of order 
errors as a reliable assessment of memory for order is inappropriate as differences in recall at an item level will lead to differences in the possible number of order errors (Saint-Aubin \& Poirier, 1999b). Lists with low item recall will likely have fewer order errors than lists where the recall level is good, as it is not possible to emit an order error if the item information associated with it has been lost. Instead any assessment of order errors must be considered in relation to the total number of items recalled. This will be achieved in Experiment 1 by using conditional probabilities, which are generated for each list by dividing the number of items recalled out of position by the total number of items recalled regardless of position.

\subsubsection{Hypotheses}

In line with Roodenrys et al. (2002), it was expected that words from large phonological neighbourhoods would be more likely to be recalled correctly than small neighbourhood words. Further, if phonological neighbourhood density does affect memory for order in the manner suggested by Roodenrys and colleagues, then more order errors would be expected for large neighbourhood words than for small neighbourhood words. It was further predicted that large neighbourhood words would be more likely to be recalled earlier than their presented position, while small neighbourhood words would be recalled later than their presented position.

\subsubsection{Method}

\subsubsection{Participants}

Eighteen undergraduate psychology students (6 males, 12 females) were recruited from the University of Wollongong, School of Psychology subject pool. All participants were native English speakers and none reported difficulties with hearing or understanding the experimental stimuli. Students who participated in the research were awarded course credit. 


\subsubsection{Stimuli}

Stimuli were 94 single syllable consonant-vowel-consonant (CVC) words selected from the Celex database (Baayen et al., 1993 - see Appendix A). Stimuli were not matched for word length as presentation and response were oral. Stimuli were used to create two conditions, with 47 words forming the dense phonological neighbourhood word pool (mean phonological neighbourhood size: 33.49 neighbours, $S D=6.96$ ), and 47 words forming the sparse phonological neighbourhood word pool (mean phonological neighbourhood size: 11.47 neighbours, $S D=3.05$ ). A t-test revealed that the difference between the large and small neighbourhood conditions was significant $t(92)=19.87, p<.001$. A t-test revealed no significant difference between conditions on word frequency, $t(92)=.99, p=.327$, or neighbourhood frequency, $t(92)=-.22, p=.827$. Although no neighbours appeared within the stimuli sets, it is important to examine whether the overall phonological diversity is the same for small neighbourhood sets and large neighbourhood sets. This could result in a greater degree of phonological confusion for one set over another, particularly if it occurs on the rime component. Phonological similarity was estimated using a custom made Microsoft Excel program that is based on Mueller et al. (2003)'s PSIMETRICA. This method produces estimates of average pairwise similarity for onset, nucleus and coda phonemes in CVC stimuli. Similarity ratings range from 0 to 1 with 1 indicating perfect similarity. T-tests revealed that the similarity measures for the nucleus and coda of each stimuli sets was not different for large and small neighbourhood words (nucleus: $t(92)=1.48, p=.143$; coda: $t(92)=-1.37, p=.176$ ). A t-test revealed that there was more similarity in the onset component for large neighbourhood words $(M=.24, S D=.05)$ than for small neighbourhood words $(M=$ $.29, S D=.05), t(92)=-4.68, p<.001$. This may induce a degree of phonological confusion for large neighbourhood words although it is not expected that this will exert an influence in mixed lists as the phonological diversity within each list will be evened out by having both large and small neighbourhood words in the same lists. Stimuli were recorded by a female speaker with an Australian accent and were digitised using SoundEdit 16. 


\subsubsection{Procedure}

The stimuli sets were combined to make 94 lists of 6 items, with neighbourhood density alternating within lists. Lists were constructed such that no word could appear more than once in any given list, and each word was presented just once in each serial position. This was done for two reasons, the first being to give a measure of how well each word was recalled in each position, and the second being that it ensured that each word appeared the same number of times in the experimental trials. Half of the lists started with a large neighbourhood word and half started with a small neighbourhood word.

Participants were tested individually in a single session lasting 1 hour. Testing was controlled using a purpose written program on a Macintosh computer. Items were played to participants through headphones at the rate on one word per second. Immediately after list presentation, participants were cued to recall the items by a short beep. Participants were instructed to verbally recall the items in the order that they had been presented, saying pass for any items they could not recall. The experimenter remained in the room throughout the experiment to record participants' responses and to ensure compliance with instructions. When participants had completed all 94 lists, they were debriefed and thanked for their participation.

\subsubsection{Results}

Recall data were scored using a correct-in-position criterion. Each word appeared once in each serial position, resulting in 6 responses generated for each word for every participant. The total number of items correctly recalled in each serial position was generated for each participant for both large and small neighbourhood sets.

The data were analysed using a 2 (neighbourhood size) by 6 (serial position) repeated measures Analysis of Variance (ANOVA). There was no significant main effect of neighbourhood density $(F(1,17)=.003, p=.955)$ indicating that overall recall level did not differ between the large and small neighbourhood sets.

There was a significant effect of serial position, $F(5,85)=89.88, p<.001$. As seen in Figure 7.1, recall declines across serial position until the last item. 


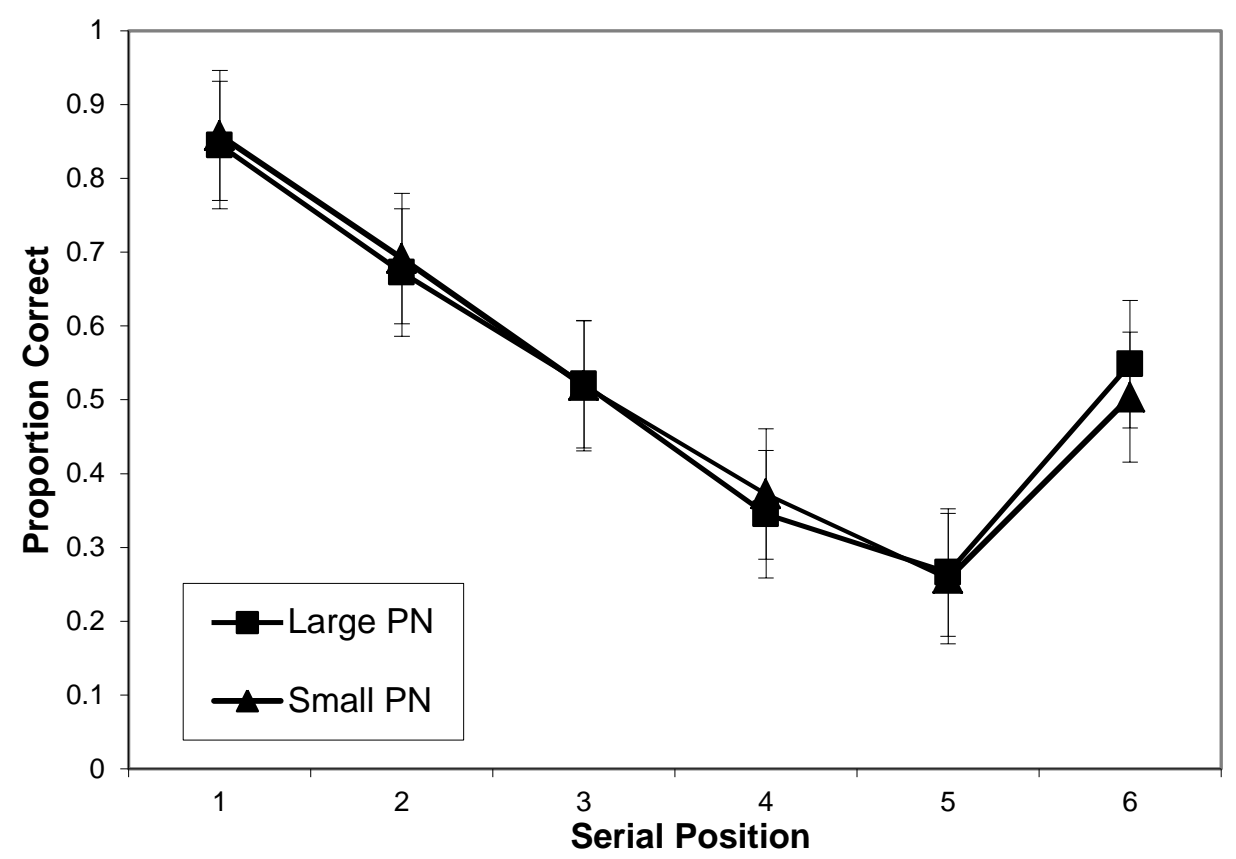

Figure 7.1. Mean serial recall performance across serial position as a function of phonological neighbourhood size.

There was no significant interaction between neighbourhood density and serial position indicating that the recall of large and small neighbourhood words was the same across serial positions, $F(5,85)=1.49, p=.201$.

\subsubsection{Order Errors}

An analysis of order errors was conducted. An order error was noted if a participant had recalled the item within the list but not in the correct serial position. The total number of order errors was summed across the entire set within each condition. Errors were then classified as to whether they were anticipation or postponement errors. Means and standard deviations are reported as mean number of each error across the whole set of lists for each condition.

A paired samples t-test revealed no significant difference in total number of order errors between large $(M=23.56, S D=11.91)$ and small neighbourhood sets $(M$ $=20.89, S D=10.57), t(17)=1.67, p=.113$.

A t-test showed no significant difference in the total number of anticipation errors between large $(M=12.11, S D=7.61)$ and small neighbourhood sets $(M=$ $11.56, S D=6.38), t(17)=.36, p=.721$. There was no difference between the total 
number of postponement errors from large $(M=11.44, S D=6.55)$ and small neighbourhood sets $(M=9.33, S D=5.66), t(17)=1.77, p=.096$.

Conditional probabilities were generated by dividing the number of order errors for each word by the number of times the word was recalled regardless of serial position (the free recall score). The average conditional probability score was generated for each participant for large and small neighbourhood sets. A pairedsamples t-test revealed a greater proportion of order errors for large neighbourhood sets $(M=.13, S D=.07)$ than for small neighbourhood sets $(M=.09, S D=.05), t(17)$ $=3.80, p<.001$. There was some concern that the assumptions of the t-test had not been met, as the data did not appear to be normally distributed. A Wilcoxon Signed Rank Test was conducted and revealed that the median difference between large and small neighbourhood sets was significant $(p<.005)$.

\subsubsection{Discussion}

Experiment 1 revealed no significant effect of phonological neighbourhood density on item memory. This is inconsistent with Roodenrys et al. (2002) who found a significant effect of neighbourhood density on item memory when items were presented in mixed lists. The current experiment differed from Roodenrys and colleagues by using a factorial manipulation of the stimuli rather than a continuous regression analysis. That is, in Roodenrys et al.'s analysis large neighbourhood words did not necessarily alternate with small neighbourhood words, while this was true in the current experiment. The polar differences between stimuli along the neighbourhood dimension within lists in the current experiment may have contributed to the absence of the phonological neighbourhood effect in this experiment. While inconsistent with the Roodenrys et al. finding, the inability to find a phonological neighbourhood effect on item memory in mixed lists is consistent with findings from the word frequency literature. Hulme et al. (2003) found a significant word frequency effect on item information when words were presented in pure lists, much like the effect typically observed with phonological neighbourhood density. This advantage for high frequency words was eliminated when words were presented in mixed lists with alternating high and low frequency words, with mixed lists recalled at an equivalent and intermediate level compared with pure lists. Pure lists were not 
examined in Experiment 1, so it is unclear whether recall of mixed lists of large and small phonological neighbourhood words follows the same pattern relative to pure lists as high and low frequency words. However, the removal of the frequency effect in the mixed list condition is replicated with phonological neighbourhood density in Experiment 1 . This potentially implicates a broader mechanism than semantic association via natural language use.

The effect of phonological neighbourhood density on order information failed to reach significance when simply counting the number of order errors. However there was an effect on order when taking into account the number of items recalled. The conditional probability analysis in the current experiment reflects the number of times each word was recalled in an incorrect position as a proportion the number of times it was recalled overall. Overall, there were more order errors for large neighbourhood words than for small neighbourhood words when considering the level of item recall. This finding is interesting given that there was no overall difference in the number of items recalled (the free recall score) between large and small neighbourhood sets, nor was there a significant difference in the number of raw order errors in large or small neighbourhood sets. Therefore, the conditional probability analysis in this experiment reflects different patterns of recall between small and large neighbourhood words. It is possible that a difference was found because a small change in the denominator (the free recall) is likely to produce a substantial change in the conditional probability score. There were very few order errors in the current experiment (approximately one every two lists) and the maximum possible denominator for each list is six. This leads to a situation where there may be an exaggeration of an order effect when analysed using conditional probabilities.

The finding of no overall difference in the number of order errors, the number of anticipation errors or the number of postponement errors is inconsistent with findings from Roodenrys et al. (2002) and with the queue-jumping hypothesis. Although a conditional probability analysis was applied in Experiment 1, order memory may have still been confounded with item memory as item information must be retrieved for an order error to occur. In supraspan lists, such as those used in Experiment 1, some of the item information is lost leading to a possible underestimation of the number of order errors, as an item cannot be recalled in the wrong position if the information pertaining to the identity of that item is missing. 
Further, anecdotal evidence suggests that participants' cognitive representation of the list may become disordered during maintenance and recall leading to loss of item information due to the time spent attempting to retrieve the order information. Therefore, a conditional probability analysis of serial recall data leads to an incomplete picture of order memory. To adjust for differences in item recall between small neighbourhood and large neighbourhood words, a different task needs to be used that does not rely so heavily on memory for items. To explore this latter possibility further, a second study was conducted that controlled for the level of item recall.

\subsection{Experiment 2: Phonological Neighbourhood Effects on Serial Recognition: Mixed Lists}

\subsubsection{Rationale}

Experiment 1 did not reveal an effect of phonological neighbourhood density on a simple analysis of order errors in the serial recall task, suggesting that, contrary to previous research, this variable does not influence memory for order. Further, while there was a significant effect on conditionalised order errors, this may be due to an exaggeration of small changes in the data set due to the small number of order errors reported and the influence of the changes in the denominator (the free recall score). As discussed previously, serial recall necessarily involves remembering both the order of items and the items themselves, and this confound may have influenced the occurrence of order errors in Experiment 1. If the effect of phonological neighbourhood density on the conditional probability analysis in the first experiment is due to a long-term memory influence rather than a limitation of ISR, then the effect should be present in a task where item memory is not paramount for successful performance. The aim of Experiment 2 was to reduce the requirement to retain items by using a serial recognition task. In serial recognition, participants are presented with a sequence of items followed by a probe list, which must be judged as same or different from the study list. The probe list contains the same items as the study list; however items may be in a different order, such that a transposition occurs between consecutive items. This task is heralded as a more accurate test of memory for order 
as there is no explicit requirement for the maintenance of item information as such information is provided at both study and test (Henson et al., 2003).

As Experiment 2 tested the queue-jumping hypothesis, mixed lists were used, with large and small neighbourhood words presented in the same list in an alternating fashion. Changes within lists occurred as transpositions between consecutive items such that either a large neighbourhood word or a small neighbourhood word was brought forward in the list and the complementary item moved back in the list.

\subsubsection{Hypotheses}

It was expected that performance on the serial recognition task would be affected by whether the changes in order are consistent or inconsistent with a participant's internal representation of the list. As Roodenrys et al. (2002) proposed that participants are more likely to recall a large neighbourhood item earlier than its presented position, it follows that changes that occur in recognition trials which result in a large neighbourhood word coming forward in the list would be more consistent with the internal representation of the list than when a small neighbourhood word comes forward. Thus it was expected that trials where a large neighbourhood word comes forward would be more difficult to detect as "different" than when a small neighbourhood word comes forward. This should be reflected as fewer correct rejections (correctly detecting a change has occurred) for lists where a large neighbourhood word comes forward. Further, there should be no difference in the mean false alarm rate for "same" $L S L S L S$ and SLSLSL lists (incorrectly saying a change has occurred when it has not).

\subsubsection{Method}

\subsubsection{Participants}

Thirty-eight undergraduate psychology students from the University of Wollongong were involved in Experiment 2 (9 male, 29 female) in exchange for course credit. These participants had not participated in Experiment 1 and were native 
English speakers. No participants reported having any difficulties hearing or understanding the stimuli.

\subsubsection{Stimuli}

The stimuli were identical to those used in Experiment 1.

\subsubsection{Procedure}

Testing was controlled using a purpose written program on a Macintosh computer. The two word sets (large and small phonological neighbourhood size) were used to create 96 lists of 6 items. Items were pseudorandomised so that large and small neighbourhood words alternated within each list. The alternation began with a large phonological neighbourhood word on half of the trials and a small phonological neighbourhood word on the other half of the trials. Lists were counterbalanced so that half of the participants received a block of SLSLSL lists first and LSLSLS lists second and vice versa.

Participants were tested individually in a single session lasting 1 hour. Each list was presented twice through a set of headphones, with the second presentation following the first after a 2 second gap. Participants were informed that both presentations would contain the same items, but that items in the second presentation may or may not be in the same order as the first presentation. If the second instance differed in order to the first it would be a transposition of two neighbouring items. Transpositions occurred on half of all trials. On lists that contained transpositions two and only two items were transposed. That is, transpositions occurred on 48 of the 96 trials, and this was distributed evenly between those starting with large and small neighbourhood words. Changes occurred with equal probability between positions 2 and 3, 3 and 4, and 4 and 5. Transpositions between adjacent items occurred such that either a large phonological neighbourhood word or a small phonological neighbourhood word was brought forward in the list with equal probability. Items were presented through headphones at a rate of 1 item per second. Participants were instructed to say same or different in response to whether the lists were presented in the same order. The examiner, who remained in the room throughout the experiment, 
recorded responses. Participants were debriefed and thanked for their participation once they had completed the experiment.

\subsubsection{Results}

Correct rejection rates (correctly detecting when a change had occurred) were collated for each change type (large PN word forward and small PN word forward) and summed across change position. Means are expressed as proportion of trials. A ttest revealed no significant difference between when a large neighbourhood word came forward $(M=.64, S D=.23)$ than when a small neighbourhood word came forward $(M=.63, S D=.22), t(37)=.12, p=.903$. A paired-samples t-test revealed no significant difference between the mean correct rejection rate for $L S L S L S$ lists ( $M$ $=.68, S D=.14)$ and $S L S L S L$ lists $(M=.73, S D=.14), t(37)=-1.73, p=.093$.

\subsubsection{Discussion}

Experiment 2 showed no effect of phonological neighbourhood density on memory for order in a serial recognition task using mixed lists of large and small neighbourhood words. This is inconsistent with Roodenrys et al. (2002) and is not in line with the queue-jumping hypothesis. The results of Experiment 2 suggest that phonological neighbourhood density does not influence memory for order, at least in the manner described by Roodenrys and his colleagues. This suggests that their result may reflect something specific about the stimuli used, and may not be generalisable to other stimuli. Alternatively, the effect may not be robust to changes in methodology or there may be insufficient power to detect the effect if it is small. While serial recognition, argued to be sensitive to differences in order memory, did not elicit an effect of neighbourhood size on influence memory for order in mixed lists in the current experiment, it may still show an influence in pure lists, in line with findings from Allen and Hulme (2006). 


\subsection{Experiment 3: Phonological Neighbourhood Density Effects in Serial Recall and Serial Recognition using Pure Lists.}

\subsubsection{Rationale}

The results of the first two experiments suggest that neighbourhood density does not influence memory for order, at least when mixed lists are used. However, these experiments do not explicate a long-term memory influence on short-term memory for order in pure lists. The aim of Experiment 3 is to explore the neighbourhood effect on memory for order in pure lists, following the results from Allen and Hulme (2006). As in Experiment 2, memory for order will be assessed using a serial recognition task. A potential problem with using a simple hit rate criterion to look at differences in performance is response bias. That is, participants may have a propensity to respond one way over another. Signal detection theory can be used to account for response bias by providing an index of the proportion of correct responses compared to responses that were incorrect, expressed as a standard deviation $\left(d\right.$ '). Higher values of $d^{\prime}$ ' indicate better recognition, or more accurate detection of when a change did or did not occur. Signal detection theory was not appropriate in Experiment 2 as LSLSLS and SLSLSL lists both contain movement of large and small neighbourhood words. Therefore, a simple analysis of anticipation and postponement errors was more suitable for the second experiment.

It is possible that the failure to find an effect in the first two experiments was due to the stimuli used. Therefore, a secondary aim of Experiment 3 was to ascertain whether the stimuli used are capable of producing the neighbourhood effect identified in the literature. A serial recall task was used to determine whether the stimuli used in the current series of experiments are able to elicit the neighbourhood effect. While this task involves the maintenance of both item and order memory, these may be separated in the subsequent analysis. Item memory was assessed via scoring using a free recall criterion, that is, an analysis of performance conducted on all items correctly recalled regardless of whether they were recalled in the correct position. Order memory was assessed in serial recall by examining the conditional probability scores of the two lists, and through an analysis of order errors. Order memory was also assessed using a signal detection analysis of serial recognition data. 


\subsubsection{Hypotheses}

It was expected that if phonological neighbourhood density affects memory for order, performance on a serial recognition task would differ between lists of large neighbourhood words and lists of small neighbourhood words. It was expected that if participants have difficulty maintaining the order of one list condition over another, they would be more likely to state that a change had not occurred when it had (a miss), and would also be more likely to state that a change had occurred when it had not (a false alarm). Similarly, if participants are better able to maintain order information in one condition over the other, they would be more likely to correctly detect when a change occurred (a correct rejection), and when a change did not occur (a hit). In line with the Allen and Hulme (2006) result in serial recall, it was expected that recognition performance would be better for large neighbourhood lists than for small neighbourhood lists, manifested as higher $d$ 'values for these sets.

It was expected that performance in the serial recall task would mimic that of previous research, with superior short-term memory for lists of large phonological neighbourhood words compared with lists of small neighbourhood words. In line with findings from Allen and Hulme (2006), it was expected that large neighbourhood lists would show higher mean conditional probability scores than lists of small neighbourhood words.

\subsubsection{Method}

\subsubsection{Participants}

Twenty undergraduate psychology students from the University of Wollongong participant pool (10 male, 10 female) participated in return for course credit. Participants were all native speakers of English and none reported any difficulties hearing or understanding the stimuli. People who had participated in Experiments 1 and 2 were excluded from participating in Experiment 3. 


\subsubsection{Stimuli}

Stimuli were identical to those used Experiments 1 and 2.

\subsubsection{Procedure}

Participants were tested individually in a single session of approximately 1 hour. Testing was controlled on a Macintosh computer using a purpose written program. Each participant completed an ISR task and a serial recognition of order task. The order of administration of the tasks was counterbalanced across participants, and within tasks, the order of presentation of either blocks of large or blocks of small neighbourhood lists was counterbalanced. Participants were given five practice lists prior to the presentation of the first experimental list in each condition. The examiner remained in the room throughout the experiment to record responses and to ensure compliance with instructions. After participants had completed all recognition and recall trials they were debriefed and thanked for their participation.

Immediate Serial Recall. The word sets were presented in their own 24 list series for each condition, with 6 items in each list. Items were randomly allocated to lists with the proviso that no item appeared twice within the same list but could appear in consecutive lists. Whether participants were presented with large neighbourhood lists or with small neighbourhood lists first was counterbalanced between participants. Participants were presented with six words via headphones at a rate of one item per second. Two seconds after the presentation of the last item in a list, participants heard a recall cue. Participants were instructed to orally recall items in the original presentation order upon hearing the recall cue. They were instructed to substitute the word pass for any items they could not recall.

Serial Recognition. The word sets were presented in their own 36 list series for each condition with six items within each list. Again, items were randomly assigned to lists with no item appearing twice within the same list but items could appear in consecutive lists. Participants were either presented with large neighbourhood lists first or small neighbourhood lists first and this was counterbalanced between 
participants. Each participant was presented with six words through a set of headphones, at a rate of one item per second. Following a two second gap, the same six items were presented again to each participant. Hence, both lists contained the same items, but the presentation order may have changed in the second presentation of the items. If the second list differed from the first it would be a transposition of two consecutive items. Transpositions occurred on half of all trials, with changes occurring with equal probability between positions two and three, three and four, and four and five. Participants were instructed to say same or different in response to whether the lists were presented in the same order.

\subsubsection{Results}

\subsubsection{Serial Recall}

Serial recall data were scored initially using a correct-in-position criterion. That is, an item was correct if the correct word was recalled in the correct serial position. These data, collapsed across participants and lists, are shown in Figure 7.2. Means and standard deviations are expressed as proportions. Serial position curves possess the typical pattern of a pronounced primacy portion and smaller recency effects. An advantage for large phonological neighbourhood lists appears in the latter serial positions, in particular from positions four to six. No difference between neighbourhood sets is apparent in serial positions one to three.

The data were analysed using a 2 (phonological neighbourhood size) by 6 (serial position) repeated measures Analysis of Variance. This revealed a significant main effect of phonological neighbourhood density, $F(1,19)=8.95, p<.008$ with overall recall accuracy greater for lists containing words from large phonological neighbourhoods $(M=.58, S E=.25)$ than from small phonological neighbourhoods $(M=.54, S E=.29)$.

As expected there was a significant effect of serial position, $F(5,95)=98.94$, $p<.001$, reflecting the serial position curve depicted in Figure 7.2. This shows that recall declines across serial positions until the last item. 


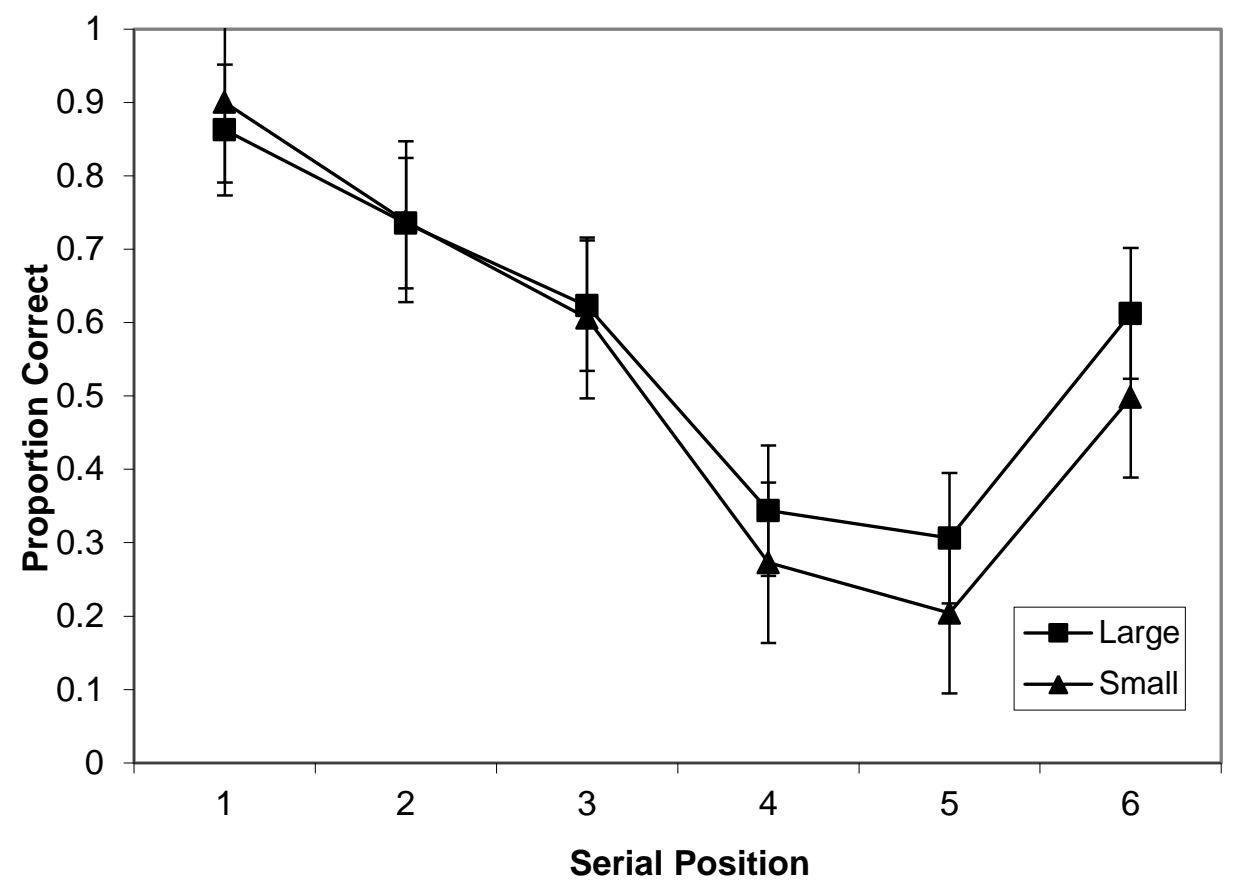

Figure 7.2: Recall performance across serial position for large and small neighbourhood lists with standard error bars.

The interaction between neighbourhood size and serial position was significant, $F(5,95)=5.43, p<.001$, showing that the recall advantage for large phonological neighbourhood words is not consistent across all serial positions. Indeed, recall appears to differ between the first and second half of the list, with an apparent advantage for large phonological neighbourhood items in the last three positions. Paired-sample t-tests were used to determine whether there was a neighbourhood effect in the first half of the list and in the second half of the list. A Bonferroni adjustment was made, giving a critical alpha level of .025. There was no significant neighbourhood effect in the first three serial positions $(p=.624)$. There was a significant advantage for large neighbourhood words in the last three positions $(p<.025)$.

The data were also analysed using a free recall criterion, where an item was scored as correct if it was reported correctly anywhere in the list. This yielded an identical pattern of results to the analysis using the correct in position criterion. That is, an item was more likely to be recalled in somewhere in the list if it was from a large neighbourhood $(M=.64, S D=.10)$ than if it was from a small neighbourhood $(M=.61, S D=.09), t(19)=2.60, p=.018$. 


\subsubsection{Error Analysis}

\section{Intrusions}

An error was classified as an intrusion if a word was erroneously recalled in place of the correct item. Means and standard deviations are reported as averages across the whole set for each condition. On average, intrusions were more likely to occur in large neighbourhood sets $(M=12.55, S D=7.56)$ than in small neighbourhood sets $(M=9.80, S D=5.33)$. A t-test revealed that this difference was significant, $t(19)=-2.80, p<.011$.

Almost half of all intrusions (49.54\%) were classified as phonological neighbourhood intrusions. That is, a phonological neighbour of the target was recalled in place of the target word. This was more common for the large neighbourhood set $(M=.63, S D=.27)$ than for the small neighbourhood set $(M=.36, S D=.30), t(19)=$ $3.87, p<.001$. These means and standard deviations reflect the proportion of intrusions that are considered neighbourhood intrusions.

\section{Order Errors}

A response was classified as an order error if it was recalled correctly within the list but in a serial position different from where it was originally presented. Order errors were further classified as anticipation errors and postponement errors. An anticipation error was recorded if the item was recalled in a position earlier than its presentation position, while a postponement error was recorded if an item was recalled later than its input position. Means reflect the average number of errors for each set.

A t-test revealed that anticipation errors were more likely to occur in small neighbourhood sets $(M=3.85, S D=2.52)$ than in large neighbourhood sets $(M=$ 2.95, $S D=2.56), t(19)=2.20, p=.041$. Occurrence of postponement errors did not differ between small $(M=4.40, S D=2.26)$ or large $(M=3.65, S D=2.50)$ neighbourhood sets, $t(19)=1.12, p=.276$. Overall, there was no difference on the total number of order errors between the large neighbourhood sets $(M=6.60, S D=$ 
4.10) and the small neighbourhood sets although the difference approached significance $(M=8.25, S D=3.92), t(19)=-1.83, p=.084$.

Conditional probability scores were generated by dividing the total number of order errors by the number of items recalled regardless of order (free recall score) (Murdock, 1976). The data revealed no significant difference between the proportion of order errors for small neighbourhood lists $(M=.12, S D=.07)$ and for large neighbourhood lists $(M=.10, S D=.06)$ at $t(19)=1.42, p=.172$.

\subsubsection{Serial Recognition}

Serial recognition data were analysed using an accuracy criterion, which was generated by summing the correct rejections (number of times participants correctly detected when a change had occurred) and the hits (number of time participants correctly detected when a change had not occurred). A t-test revealed no significant difference between the mean accuracy score for large neighbourhood lists $(M=.71$, $S D=.11)$ and small neighbourhood lists $(M=.70, S D=.07), t(19)=-.46, p=.653$.

The data were subject to further analysis using the principles of signal detection theory, to control for response bias. A d prime ( $d$ ') value was generated for each participant by first calculating the $\mathrm{z}$ statistic for the hit rate (proportion of 'same' trials to which the participant responded 'same') and for the false alarm rate (proportion of 'same' trials to which the participant responded 'different'). The $d$ ' statistic was given by subtracting the z-score for the false alarm from the z-score for the hit rate (Green \& Swets, 1966). Means reported here are mean $d$ '. A t-test conducted on the $d$ ' statistic revealed no significant difference in performance between large $(M=1.36, S D=.80)$ and small $(M=1.12, S D=.45)$ neighbourhood sets, $t(19)=-1.55, p=.137$.

\subsubsection{Discussion}

Experiment 3 showed an advantage in correct recall for large neighbourhood words over small neighbourhood words in ISR, which was consistent with the hypothesis and with past research. This is even with less phonological diversity on the onset component for large neighbourhood words, which, as discussed earlier, could 
result in greater phonological confusion for these items. This would work against the neighbourhood effect, and may suggest that the magnitude of the effect here is smaller than what would be expected if there were greater diversity in initial phoneme of the large neighbourhood words used in this experiment.

It has previously been argued (Roodenrys et al., 2002) that the phonological neighbourhood effect reflects redintegration processes that operate to restore lost information to a degraded item. According to this account, recall will be better for large neighbourhood words than for small neighbourhood words as the former receive more facilitative activation from their greater number of neighbours. Importantly, the presence of a neighbourhood effect in Experiment 3 refutes the suggestion that the inability to find a neighbourhood effect in the first two experiments is due to the stimuli used.

While the finding of a neighbourhood effect is in line with Roodenrys et al. (2002), there was a novel finding of an interaction between neighbourhood size and serial position. This is reflected in the lack of effect in the first three serial positions, with an advantage for large neighbourhood words evident in the last three serial positions. This serial position interaction may be due to an increase in degradation of the items across serial positions. As redintegration operates on degraded items, the more degraded the traces, the more correct recall becomes dependent on redintegrative processes (Cowan, 1992). As the phonological neighbourhood effect is attributable to redintegration, it is probable that the effect will be attenuated when degradation is minimal, which is typical of earlier serial positions. The superior memory for the first few items can be, in part, attributed to selective rehearsal for these items. Previous studies have shown that the first few items in a list tend to receive the most rehearsals (Rundus, 1971). Further, Tan and Ward (2000) suggested that the first two items tend to be distinctive, leading to superior recall of these items and an attenuation of the neighbourhood effect. Moreover, this pattern of results is not exclusive to this experiment, with previous researchers showing similar interactions with concreteness and serial position (Walker \& Hulme, 1999) and with frequency and serial position (Hulme et al., 1997).

While the results regarding item memory are consistent with the hypotheses and follow a similar pattern to previous research, the results regarding memory for order were not. In Experiment 3, memory for order was examined using a serial 
recognition task as well as an order analysis of the serial recall data. There was no significant effect of neighbourhood density in the serial recognition task. Further, there was no significant difference between the large and small neighbourhood lists on the raw number of order errors, although it approached significance when using an absolute criterion. However, using conditional probabilities, which provides a more stringent test of order memory, the effect was not significant, although it was in the predicted direction. This is contrary to findings of a significant effect of neighbourhood density on conditionalised order errors in Experiment 1 and suggests that the effect in the first experiment may have been due to the over-sensitivity of the conditional probability analysis to small changes in the data. While the conditional probability analysis was not significant, there was a significant difference between large and small neighbourhood sets on the number of anticipation errors, with small neighbourhood words more likely to be recalled ahead of their serial position than large neighbourhood words. This may reflect the fact that recall is poorer for small neighbourhood words and, where an item is lost the remainder of items are recalled at $n-1$. In addition to assessing memory for order in the serial recall task, the process was also assessed using serial recognition. The lack of significant effect in the serial recognition task or on the order examination of the serial recall task is in contrast to findings from Roodenrys et al. (2002) and Allen and Hulme (2006).

The reason for the discrepancy from Roodenrys et al. (2002) may be because this experiment used pure lists of large and small neighbourhood words whereas Roodenrys et al. (2002) experiment used mixed lists of small and large neighbourhood words. Given the argument put forward by those authors that large and small neighbourhood words in the list compete in some way at output, causing large neighbourhood words to 'jump the queue' it is perhaps not surprising that no effect was obtained in pure lists. However, Allen and Hulme (2006) did report an effect in pure lists. There are a number of minor differences between Allen and Hulme's (2006) paper and the current experiment. Allen and Hulme used seven item lists rather than the six item lists used in this experiment. Despite this, the pattern of performance across serial positions between the two experiments is almost equivalent, although there is a slightly smaller recency effect in the current experiment. Using seven item lists gives more room for items to move. By increasing the list length by even one item, there is an increase in number of possible order errors. 
In addition to the increased list length, Allen and Hulme (2006) used stimuli that differed on two dimensions rather than just on neighbourhood size. There were only 16 words in each condition whereas the current experiment there were 47 words in each condition. Therefore for the Allen and Hulme experiment, there were only 32 large neighbourhood words (which were then divided into high and low frequency conditions), and 32 small neighbourhood words (again which were divided into high and low frequency conditions). Utilising smaller item sets reduces the reliance on item information, and is proposed to make the task more one of memory for order (Saint-Aubin \& Poirier, 1999b). While a 32 item set is still relatively large compared to those discussed by Saint-Aubin and Poirier (1999b), Allen and Hulme (2006) presented lists within each stimuli condition which will have effectively reduced the item set to 16 .

While these differences seem minor, they may be sufficient to elicit an effect on memory in Allen and Hulme's (2006) study and not in the current studies if the effect is weak. In addition, while the conditional probabilities were larger in Allen and Hulme's (2006) experiment than in the current experiment, they were still quite small considering the respective magnitude of item level effects.

The inability to find a significant effect in this experiment may be due to the lack of sensitivity of the serial recognition task. In order to give an erroneous response, the presentation of the second list must be inconsistent with the participants' internal representation of the first list. That is, it was proposed that if participants make an order error in their internal representation of the list, then they should incorrectly respond "different" when the two presentations were the same, or should respond "same" if the erroneous internal representation matched the transposition made on the second list. However, if participants' internal representation of the list has an order error, but this is not a transposition between two items, or it was a transposition between two items that were not in fact transposed in the presentation, then they may correctly respond that the lists were different even though they are not detecting the order error actually presented. This makes the task potentially unreliable as a measure of order memory, as it may not be sensitive to detecting the full range of participants' responses. 
Chapter Seven: Investigating the Phonological Neighbourhood Effect on Short-Term Memory for Order

\subsection{General Discussion}

The first series of experiments revealed a phonological neighbourhood effect on a correct-in-position criterion in an ISR task using pure lists, replicating results found in previous experiments (Roodenrys et al., 2002; Allen \& Hulme, 2006). This confirms the variable's influence on item memory previously reported in the literature. However, memory for order was shown to be unaffected by phonological neighbourhood density, as evidenced by equal performance for large and small neighbourhood lists on a serial recognition task, and no influence of neighbourhood density on the proportion of order errors in ISR using pure lists. The significant effect of neighbourhood size on conditional probabilities in mixed lists was suggested to be an artefact of the interaction between the small number of order errors and small changes in the level of item recall. As the numbers are close to zero, a conditional probability analysis magnifies these small differences, which would otherwise be considered non-significant. At best, the results of these experiments suggest that if there is an effect it is small and not robust to changes in methodology.

It may be that the tasks are not adequate to assess memory for order, with the serial recognition task perhaps not sensitive enough to the order errors participants are likely to make, and the recall task still involving a confound with memory for items. In order to answer the question of whether neighbourhood size does affect memory for order a different task needs to be utilised that allows an examination of the internal representation of the lists. A serial reconstruction task can be used for this as it gives more insight to participants' internal representation of the list while reducing the reliance on memory for items. 


\section{Chapter Eight}

\section{The Phonological Neighbourhood Effect on Short-Term Memory for Order: Serial Reconstruction}

\subsection{Introduction}

The first phase of experiments examined the effect of phonological neighbourhood density on memory for items and memory for order using immediate serial recall and serial recognition respectively. Consistent with Allen and Hulme (2006), a significant effect of neighbourhood density was observed on the correct-inposition analysis of pure lists, with superior performance for large phonological neighbourhood words over small phonological neighbourhood words. Contrary to findings from Roodenrys and his colleagues (2002), no effect of neighbourhood density was observed on the correct-in-position analysis of mixed lists.

With regards to memory for order, the results so far are inconsistent with the queue-jumping hypothesis, with no neighbourhood effect on memory for order on mixed lists in serial recognition or on a raw analysis of order errors in serial recall. There was a significant effect of neighbourhood density on the conditional probability analysis of ISR performance, but this was attributed to limitations of the analysis due to the small number of items recalled and order errors. Further, the results so far suggest that while long-term memory influences short-term item memory, it has no influence on short-term memory for order, with no effect of neighbourhood density in pure lists. This is contrary to the findings of Allen and Hulme (2006).

However, it is possible that the serial recognition task used in the first phase of experiments was not sensitive enough to detect order errors naturally occurring in short-term memory. The effect on memory for order is likely to be a small one given that Roodenrys et al. (2002) reported that only around 9\% of items were recalled in the incorrect position. As the effect is small, it is unlikely that it would be observable on each instantiation of the experiment. Further, it is possible that the results from ISR reflect the fact that the relative level of item recall and number of order errors 
was small, as, even with a conditional probability analysis this task is still sensitive to the influence of item memory.

A task that overcomes the limitations identified with serial recognition and serial recall must be employed to ascertain whether phonological neighbourhood density affects memory for order. The task must reduce the requirement to maintain item information while enabling participants to make order errors that naturally occur in the memory process. To address these issues, the second phase of experiments employed serial reconstruction as a test of memory for order. In a reconstruction task, a series of items is presented, and at test the same items are visually presented in a random order. The items remain available to the participant until reconstruction is complete. As the item information is given, the participants only need to recreate the original order of presentation. Serial reconstruction has been proposed as a process pure test of order memory (Whiteman et al., 1994), and although objections to this claim have been made (Neath, 1997), the task can be regarded as a more pure test of memory for order than other memory tasks.

\subsection{Experiment 4: The Phonological Neighbourhood Effect in a Serial Reconstruction Task: Mixed Lists}

\subsubsection{Rationale}

The aim of Experiment 4 was to test the queue-jumping hypothesis and to attempt to replicate the finding from Roodenrys et al. (2002). The first phase of experiments showed no significant effect of phonological neighbourhood density on memory for order using either a raw analysis of errors from serial recall (Experiment 1) or serial recognition data (Experiment 2) when mixed lists were used. An effect on order memory may have been masked in these initial experiments by confounds with item memory (Experiment 1) or a lack of sensitivity to naturally occurring order errors (Experiment 2). The purpose of Experiment 4 was to address these issues by assessing memory for order using serial reconstruction. In this experiment, participants were visually presented with items and were asked to indicate the original order of presentation by writing the items on paper. This was done to avoid confounds with accent as the experiments were planned in Australia but conducted in the United 
Kingdom. In line with Experiments 1 and 2, large and small neighbourhood words were arranged in an alternating fashion in the same lists.

\subsubsection{Hypotheses}

In line with Roodenrys et al. (2002) it was expected that large phonological neighbourhood words would be ordered ahead of their position more often than small neighbourhood words. This was expected to manifest as a pattern of anticipation and postponement errors such that large neighbourhood words would have a higher rate of anticipation errors whereas small neighbourhood items would have a greater amount of postponement errors.

\subsubsection{Method}

\subsubsection{Participants}

Participants were 18 members of the University of York community (5 Males, 13 Females). All participants were native English speakers and had normal or corrected to normal vision. Participants received either course credit or payment for their participation.

\subsubsection{Stimuli}

Stimuli used in Experiment 4 were identical to those used in previous experiments.

\subsubsection{Procedure}

Due to software limitations, a pool of 94 stimuli lists were created and of these 48 lists were randomly selected for each participant. The stimuli lists were created from the 94 stimuli (47 large and 47 small neighbourhood words) used in previous experiments. Lists were created such that half of the lists began with a large neighbourhood word and the other half with a small neighbourhood word. No word 
appeared more than once within each list and each word appeared just once in each serial position. Of the 48 lists selected for each participant, twenty-four began with a large neighbourhood word and twenty-four with a small neighbourhood word. The order of presentation was blocked and was counterbalanced across participants. A purpose-written program was created using Runtime Revolution to run the experimental trials using a PC.

Participants were tested individually in a single session lasting 40 minutes. Items were presented visually on a 15-inch visual display unit (VDU) as 48-point white text against a black background. Item presentation was at the rate of one word per second. Following presentation of the entire list, a row of question marks (???) appeared, which was replaced after 1 second by the reappearance of all 6 list items. These items were arranged vertically on the screen in a new and random order. Participants were instructed to reconstruct the original presentation order of the items by writing them on paper. Participants were given strict serial reconstruction instructions, that is, they were informed to begin reconstruction from the first item presented and to proceed sequentially. Participants were required to place all six items in order before proceeding to the next trial. The experimenter remained in the room throughout the experiment to ensure compliance with these instructions. On completion of the experiment, participants were debriefed and thanked for their participation.

\subsubsection{Results}

As all items were presented at test, a response was recorded for each item and was classified by whether it was in the correct position, recalled earlier than its original position, or recalled later than its original position. Items placed in the correct serial position were analysed by neighbourhood condition across serial position. A 2 (phonological neighbourhood size) by 6 (serial position) repeated measures Analysis of Variance (ANOVA) was used to analyse the data. There was no main effect of phonological neighbourhood density, $F(1,17)=.38, p=.547$, indicating that overall performance did not differ between the large and small neighbourhood sets.

There was a significant effect of serial position $F(5,85)=14.86, p<.001$. This is shown in Figure 8.1, and represents a typical serial position curve with a decrease in 
performance until the final item.

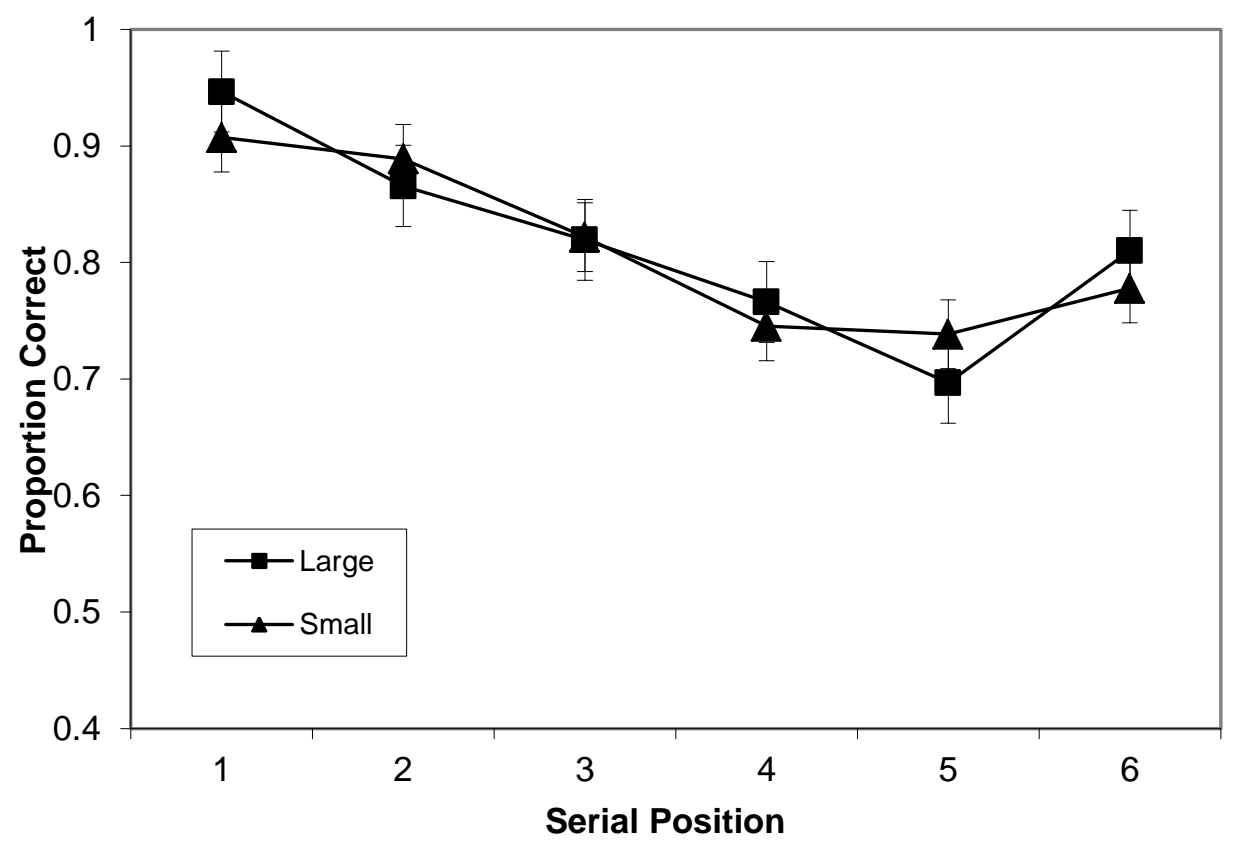

Figure 8.1. Mean number of items correctly ordered by serial position for alternating lists of large and small phonological neighbourhood words with standard error bars.

Finally, there was no significant interaction between neighbourhood set and serial position, indicating that the two conditions changed across serial position in the same way, $F(5,85)=1.47, p=.208$.

\subsubsection{Error Analysis}

An order error was noted if a participant had written a word in an incorrect serial position. The error data were averaged across trials and were used to generate mean number of anticipation and postponement errors for each participant. The resulting data were analysed using paired samples t-tests. Means and standard deviations are expressed as the proportion of the number of possible errors.

There was no significant difference between the mean number of times a small neighbourhood word was recalled later in the list $(M=.09, S D=.04)$ than when a large neighbourhood word was recalled later in the list $(M=.08, S D=.06), t(17)=$ $.32, p=.751$. 
Similarly, there was no significant difference in the mean number of anticipation errors between the two sets, $t(17)=-.31, p=.759$. This indicated that the number of times a large neighbourhood word was recalled earlier in the list $(M=.09$, $S D=.06$ ) was not significantly different from the number of times a small neighbourhood word was erroneously recalled earlier in the list $(M=.09, S D=.07)$.

Paired sample t-tests also revealed that the number of anticipation and postponement errors did not differ for large neighbourhood sets $(t(17)=-1.31, p=$ $.208)$, or for small neighbourhood sets $(t(17)=-.47, p=.648)$. This indicated that, for both large and small neighbourhood sets, a word was just as likely to be reported ahead of its position as it was to be recall later than its correct serial position.

\subsubsection{Discussion}

Experiment 4 confirmed results from Experiments 1 and 2 that there is no significant effect of phonological neighbourhood size on order information using mixed lists. The finding from Roodenrys et al. (2002) of a significant effect of neighbourhood density on mixed lists may be specific to the stimuli or the experimental methods used.

While the current experiment fails to support the queue-jumping hypothesis, it does not necessarily indicate that long-term memory does not influence memory for order. It is possible that one neighbourhood condition is more prone to order errors than the other but it may be that this effect is masked by using mixed lists, as the more unstable items in terms of order are being supported by the more stable items. Allen and Hulme (2006) found evidence that memory for order was better for lists of large neighbourhood words than for lists of small neighbourhood words. If long-term memory is able to influence the item-to-position associations, then the superior order memory for words from large neighbourhoods may be acting as anchors for small neighbourhood words. To examine this further, the effect identified by Allen and Hulme (2006) needs to be confirmed. This effect was not replicated in Experiment 3 in either the recognition task or the conditional probability analysis of order errors from serial recall. The problems with these tasks have been noted, and can be addressed by examining performance on a serial reconstruction task. 


\subsection{Experiment 5: The Phonological Neighbourhood Effect in a Serial Reconstruction Task: Pure Lists}

\subsubsection{Rationale}

The aim of Experiment 5 was to explore the significant effect of neighbourhood density on memory for order identified by Allen and Hulme (2006) using pure lists. If neighbourhood size is able to contribute to the stability of an itemto-position association then it would be expected that lists of large neighbourhood words would be remembered better than lists of small neighbourhood words. While such an effect was not identified in pure lists in Experiment 3, this may be due to task demands and lack of task sensitivity as discussed previously. Experiment 5 aimed to test this possibility by presenting participants with pure lists of large and small phonological neighbourhood words in the reconstruction task identical to that used in Experiment 4.

\subsubsection{Hypotheses}

In line with Allen and Hulme (2006) it was expected that there would be a significant effect of phonological neighbourhood density on the reconstruction performance of high and low neighbourhood lists. Specifically, it was expected that reconstruction would be better for lists of large neighbourhood words than for lists of small neighbourhood words, reflecting better order memory for these items.

\subsubsection{Method}

\subsubsection{Participants}

Participants were 22 members of the University of York community (8 Males, 14 Females). All participants were native English speakers and had normal or corrected to normal vision. People who had participated in Experiment 4 were unable to participant in Experiment 5. Participants received either course credit or payment for their participation. 


\subsubsection{Stimuli}

The stimuli used in Experiment 5 were identical to those used in the previous experiments.

\subsubsection{Procedure}

As in Experiment 4 a pool of stimuli lists were created by randomly allocating stimuli words into lists of 6 items with neighbourhood density consistent within lists. Items appeared once in each serial position and no item appeared more than once in a list. This resulted in 47 lists for each neighbourhood condition (94 lists in total). Of these 94 lists, 24 lists for each neighbourhood condition (a total of 48 lists overall) were randomly selected for each participant.

Participants were tested individually in a single session lasting 30 minutes. The procedure was identical to Experiment 4. The experimenter remained in the room to ensure adherence with these instructions. Participants commenced a new trial by selecting 'new trial' from the pop-up menu. Following presentation of all trials, participants were debriefed and thanked for their participation.

\subsubsection{Results}

As all items were presented at test, a response was recorded for each item based on whether it was recalled in the correct position, recalled earlier than its original position, or recalled later than its original position. Means are reported as proportion of the number of trials. Items placed in the correct serial position were analysed by neighbourhood condition across serial position. A 2 (phonological neighbourhood size) by 6 (serial position) repeated measures Analysis of Variance was conducted.

There was a significant main effect of phonological neighbourhood density, $F$ $(1,21)=8.90, p=.007$, with superior reconstruction for large neighbourhood lists $(M$ $=.78, S D=.15)$ over small neighbourhood lists $(M=.73, S D=.19)$.

The serial position variable violated the sphericity assumption $(W(14)=.05, p$ $<.001)$. The resulting analysis for this interaction was adjusted using the Greenhouse- 
Geisser statistic. There was a significant effect of serial position, $F(2.38,50.04)=$ $31.01, p<.001$. This reflects a decline in performance across the list until the last item, as shown in Figure 8.2.

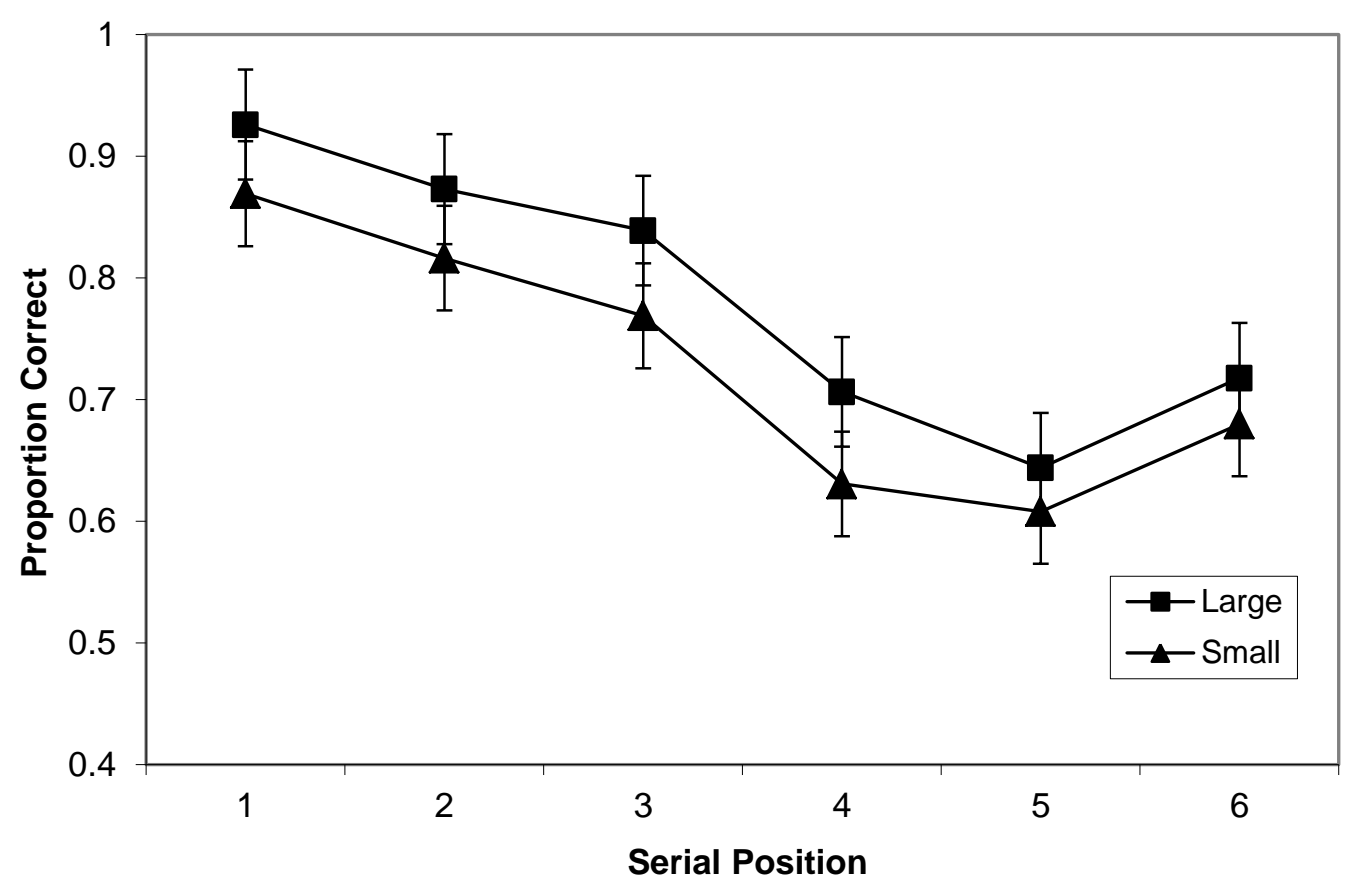

Figure 8.2. Reconstruction performance across serial position by phonological neighbourhood size with standard error bars.

There was no significant interaction between phonological neighbourhood density and serial position which indicated that the size of the neighbourhood effect did not vary across serial position, $F(5,105)=.52, p=.759$.

\subsubsection{Discussion}

Experiment 5 revealed a significant effect of phonological neighbourhood density, with performance on the order reconstruction task better for lists of large neighbourhood words than for lists of small neighbourhood words. This is consistent with Allen and Hulme's (2006) finding of better order memory for large than for small neighbourhood words in a serial recall task. The finding is suggestive of a link with long-term memory occurring at a point prior to that suggested by existing conceptions of redintegration. That is, the result is inconsistent with models that posit an account of redintegration that only plays a role during output, after the order of 
sequences has been retrieved. For instance, Page and Norris' (1998) primacy model asserts that items are retrieved in order, and only then undergo redintegration to retrieve any lost information and are then output.

There are three possible explanations of the current results. First, it may be that a redintegration-like process occurs at a point earlier than at output. Second, there may be a separate, non-redintegrative long-term memory link that assists with the maintenance of order information during retention. Third, there may be a long-term memory link that occurs during encoding and acts to strengthen the representation of certain words. One explanation that pin-points the locus of the effect to encoding is the order-encoding hypothesis (DeLosh \& McDaniel, 1996). This assumes that there is a trade-off between item and order encoding such that where item information is easy to encode more resources will be available for order encoding. This will lead to better order memory for these items. It is counterintuitive that large phonological neighbourhood words would result in better order memory according to this hypothesis, as large neighbourhood words are associated with slower and less accurate perception than small neighbourhood words. However, if the time course of perception does not influence the resources available for encoding then it is possible that, once recognised, item information pertaining to large phonological neighbourhood words is encoded easier in working memory than that pertaining to small neighbourhood words. This would result in more resources available for encoding the order information of large phonological neighbourhood words. Alternatively, if rehearsal is considered as cyclical recall of the items, then given that large phonological neighbourhood words are recalled with fewer errors than small neighbourhood words, more order recoding resources may be available for large neighbourhood words as fewer resources are necessary to re-code item information for these items during rehearsal.

Another possibility is that there are stronger inter-item associations between certain words, due to their higher level of co-occurrence in language (Sumby, 1963; Deese, 1960). This has been used as an explanation for word frequency effects, with the premise that frequent words are more likely to appear alongside other frequent words in language and this association can bolster recall of these words (Hulme et al., 2003). According to this co-occurrence hypothesis, when a word is presented in a list its activation spreads to other items in the list via connections between the items in 
long-term memory. Items that already share strong connections in long-term memory, such as items that co-occur often in language, will have a greater level of pre-existing activation that will assist in recall (Miller \& Roodenrys, 2012). The co-occurrence approach bears similarity to the semantic binding hypothesis (Patterson, Graham, \& Hodges, 1994) where patterns of phonemes become associated with one another and to a semantic representation due to a high level of co-occurrence between all of these components in speech perception and production. This semantic association acts as an adhesive for the constituent phonemes to one another and to a word. The semantic binding hypothesis addresses how phonemes are constrained within a word while cooccurrence is used to explain how words are constrained within a list. According to this account encoding will be stronger for words that naturally co-occur in language. Words from large phonological neighbourhoods or their neighbours may be more likely to co-occur with other large neighbourhood words or their neighbours. This leads to a pattern of supportive activation amongst the words in a list that may be assisting order encoding for large neighbourhood words, although exactly how this occurs is not clear. However, existing models of memory for order could account for neighbourhood effects if these pre-existing associations that act to maintain items in a relatively active state were assumed. This will be examined in more detail in Chapter 12.

The item co-occurrence hypothesis and the order-encoding hypothesis make important predictions for the comparative pattern of performance across mixed lists and pure lists. Both hypotheses would predict that performance on mixed lists would result in a smooth serial position curve that falls between the performance level of pure lists of large and small neighbourhood words. For the co-occurrence hypothesis this occurs because large and small neighbourhood words have an intermediate level of association strength, which exerts an influence over the entire list via a general level of supportive activation (Hulme et al., 2003). The order-encoding hypothesis would predict that the order encoding of large neighbourhood words would be disrupted when presented in mixed lists (relative to pure lists). Conversely, the order encoding of small neighbourhood words would be enhanced when presented in mixed lists (relative to pure lists) (McDaniel, DeLosh, \& Merritt, 2000). This would manifest as equivalent order memory for these items. Note that while the two approaches will predict the same pattern of results, the underlying cause of the effect 
is different. The co-occurrence hypothesis operates as a list-level effect, while the order-encoding hypothesis is an item-level effect.

Although the presence of a smooth serial position curve would not elucidate whether a list-level or item-level effect was in operation, a saw-toothed serial position curve for mixed lists would be suggestive of an item-level effect. That is, if words are recalled without influence from other list items then recall of a large neighbourhood word that is sandwiched between two small neighbourhood words should be the same as if it were amongst other large neighbourhood words. An argument of this sort comes from Hulme et al. (2003) who examined the effect of list composition on the word frequency effect. They found that high and low frequency words were recalled at a consistent and intermediate level compared with pure lists, supporting the listlevel hypothesis. To investigate whether this is consistent with findings on phonological neighbourhood density, performance differences between the mixed list paradigm and the pure list paradigm in Experiment 4 and Experiment 5 were examined.

\subsubsection{Combining Experiment 4 and Experiment 5}

The data from Experiment 4 and Experiment 5 were collated and analysed using a 2 (large v small neighbourhood) by 2 (mixed v pure lists) by 6 (serial position) split-plot factorial Analysis of Variance. List composition was entered as a between subjects variable while neighbourhood size and serial position were within subjects variables.

There was a marginally significant main effect of composition (pure $\mathrm{v}$ mixed lists), with a trend for overall reconstruction performance better for mixed lists ( $M=$ $.82, S D=.16)$ than for pure lists $(M=.76, S D=.17), F(1,38)=3.23, p=.080$.

A significant effect of phonological neighbourhood density was found, with overall performance better for words from large neighbourhoods $(M=.80, S D=.16)$ than from small neighbourhoods $(M=.77, S D=.18), F(1,38)=7.69, p=.009$.

The serial position variable violated the sphericity assumption $(W(14)=.05, p$ $<$.001). The resulting analysis for this effect was adjusted using the GreenhouseGeisser statistic. The main effect of serial position was significant, $F(2.30,87.45)=$ 
43.04, $p<.001$. This effect is depicted in Figure 8.3 and reflects the typical serial position curve with a decline in performance until the last item.

The neighbourhood by position variable violated the sphericity assumption $(W(14)$ $=.50, p=.034)$. The resulting analysis for this interaction was adjusted using the Greenhouse-Geisser statistic. The neighbourhood by position interaction was not significant, $F(3,149.97)=1.31, p=.269$, showing that performance on large and small neighbourhood lists changes across serial position in the same way.

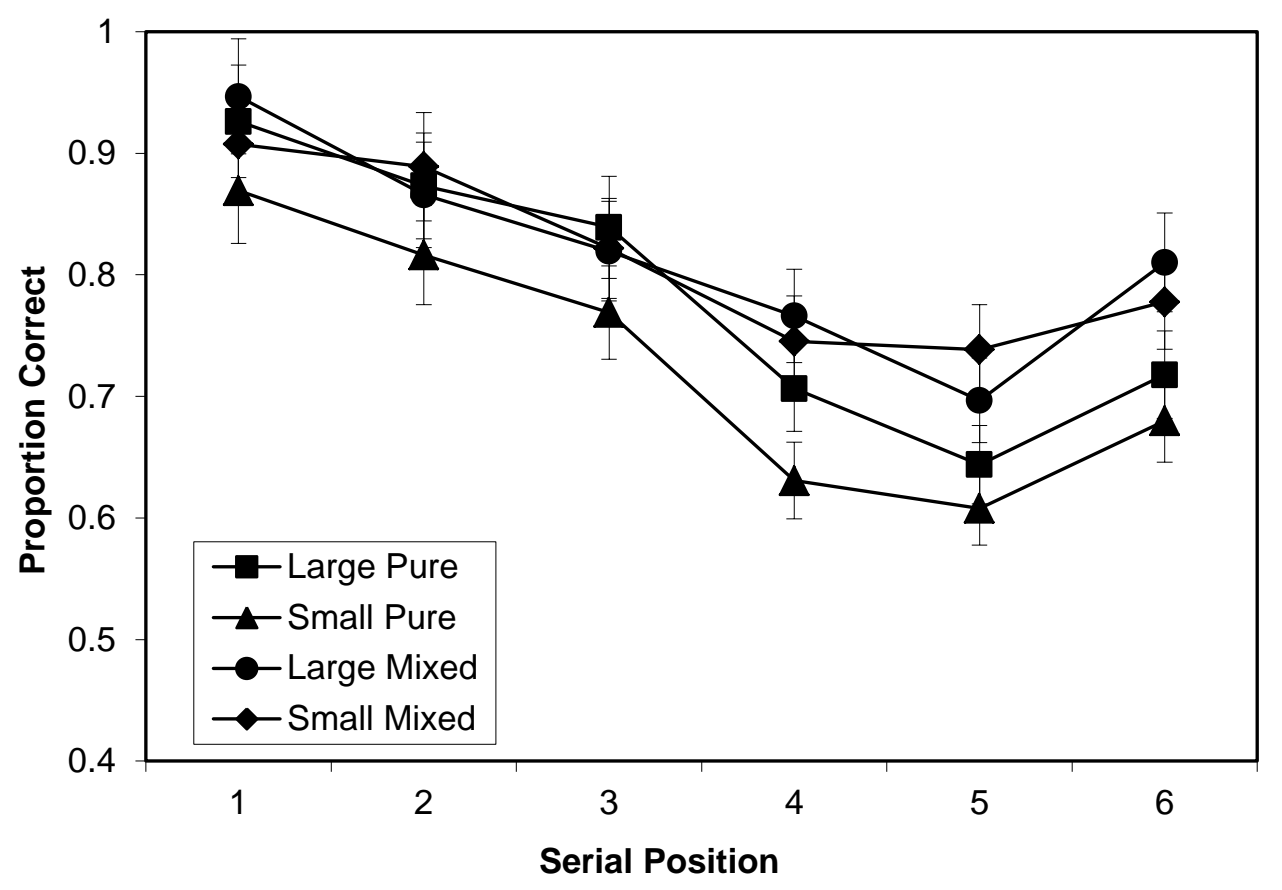

Figure 8.3. Serial reconstruction performance by neighbourhood size and list condition across serial position

There was a significant condition by neighbourhood interaction indicating that the difference in recall level for large and small neighbourhood words is not the same for pure and mixed lists, $F(1,38)=5.67, p=.022$. This reflects the finding of no effect of phonological neighbourhood density in mixed lists (Experiment 4) but a difference in performance between large and small neighbourhood lists in pure lists (Experiment 5).

The condition by position interaction was not significant, $F(5,85)=1.67, p=$ .146. That is, the pattern across serial positions is the same for pure lists as it is for alternating lists. Finally the three way interaction between condition, phonological neighbourhood density, and serial position was not significant, $F(5,190)=.87, p=$ 
.500. This shows that the difference is the neighbourhood effect between pure and mixed lists did not change across serial position.

\subsubsection{Discussion}

The purpose of conducting a combined analysis on data from Experiment 4 and Experiment 5 was to ascertain whether the effect of neighbourhood density on memory for order could be explained by either the co-occurrence hypothesis or the order-encoding hypothesis, or was due to an effect occurring at the word level. An effect occurring at the word level would be indicated by mixed list performance showing a saw-toothed serial position curve.

Looking at Figure 8.3 it appears that both large and small neighbourhood words in mixed lists are recalled as well as large neighbourhood words in pure lists for the first three serial positions. After this, performance for both the large and small neighbourhood words in the pure lists drops down to follow the usual serial position curve, but the performance in the mixed lists does not decrease as sharply. It would seem that performance on both large and small phonological neighbourhood words is better in mixed lists than in pure lists at least for the last three serial positions. This refutes both the order-encoding hypothesis and the co-occurrence hypothesis, which would suggest performance on mixed lists would occur at an intermediate level to pure lists. Instead, it appears that large neighbourhood words are helping to constrain the order of small neighbourhood words.

It is possible that the failure to support these hypotheses is due to the use of a between-subjects design, which increases the error variance. This is also, while unlikely, a potential explanation of the differences in overall performance level between Experiment 4 and Experiment 5. To investigate the effect of betweensubjects variance, performance on serial reconstruction using mixed and pure lists should be examined using a within-subjects design. 


\subsection{Experiment 6: Phonological Neighbourhood Effect in a Serial Reconstruction Task using Mixed and Pure Lists}

\subsubsection{Rationale}

The second phase of Experiments has so far indicated that phonological neighbourhood density exerts an influence in pure lists (Experiment 5), but has no effect in mixed lists (Experiment 4). An analysis of performance with list composition as a between subjects variable suggests that words in mixed lists are recalled just as well as large phonological neighbourhood words in pure lists. This may be because large neighbourhood words are acting as anchors for small neighbourhood words. In serial recall this would suggests that the effect occurs at the level of the whole list rather than being an item-level effect, however in reconstruction this does not rule out the possibility that that the effect occurs at the item level, as better coding of the absolute position of large neighbourhood words would necessarily constrain the serial position of small neighbourhood words in mixed lists.

Regardless, the pattern of performance in the between-subjects analysis did not conform to the expectation of the co-occurrence account or with the orderencoding account with performance on mixed lists surpassing that of pure lists of large neighbourhood words, at least at the terminal serial positions. To ascertain whether these findings can be attributed to individual differences, Experiment 6 was conducted which examined serial reconstruction performance with mixed and pure lists in the same participants.

\subsubsection{Hypotheses}

The results of Experiment 6 were expected to follow the same pattern of those generated by Experiment 4 and Experiment 5. That is, it was expected that performance on the mixed lists would show no effect of neighbourhood density on the mean number of order errors, including postponement and anticipation errors. Performance on pure lists was expected to mimic results from Experiment 5, with differences expected between large and small neighbourhood sets such that 
reconstruction would be better for large neighbourhood lists than for small neighbourhood lists.

\subsubsection{Method}

\subsubsection{Participants}

Participants were 22 undergraduate psychology students (4 male, 18 female) at the University of Wollongong. Participants received course credit in return for participation. Those who had participated in the previous experiments were not eligible to participate in Experiment 6. All participants were native English speakers and had normal or corrected to normal vision.

\subsubsection{Stimuli}

The stimuli used in Experiment 6 were identical to those used in the previous experiments.

\subsubsection{Procedure}

Stimuli were randomly allocated into lists of 6 words forming two pure list conditions (large phonological neighbourhood and small neighbourhood lists) and two mixed lists conditions ( $L S L S L S$ and $S L S L S L$ ) where neighbourhood size alternated within the lists. There were 24 lists for each condition, giving 96 lists overall. Lists were created to ensure that no item appeared more than once in each list, but items could appear without restriction on subsequent lists. Order of presentation of the lists was counterbalanced between participants and new lists were created for each participant.

Participants were tested individually in a single one hour session. Testing was controlled using a purpose written program on a Macintosh computer. List condition was blocked by list composition and neighbourhood size, and presentation order was counterbalanced across participants. Apart from the stimuli used, Experiment 6 followed the same experimental protocol as Experiment 4 and Experiment 5. 


\subsubsection{Results}

Data were scored using the correct in position criterion, that is, for a word to be considered correct it must be in the correct serial position. These data were averaged across 24 lists for each list condition for large and small neighbourhood words. The resulting data were subject to a 2 (neighbourhood size) by 2 (list type) by 6 (serial position) repeated measures Analysis of Variance.

There was no significant main effect of list type, $F(1,21)=1.26, p=.274$. This means that there was no significant difference in overall reconstruction performance between mixed and pure lists.

There was a significant effect of phonological neighbourhood density with words from large phonological neighbourhoods reconstructed better overall $(M=.70$, $S D=.03)$ than words from small phonological neighbourhoods $(M=.65, S D=.03), F$ $(1,21)=5.90, p=.024$.

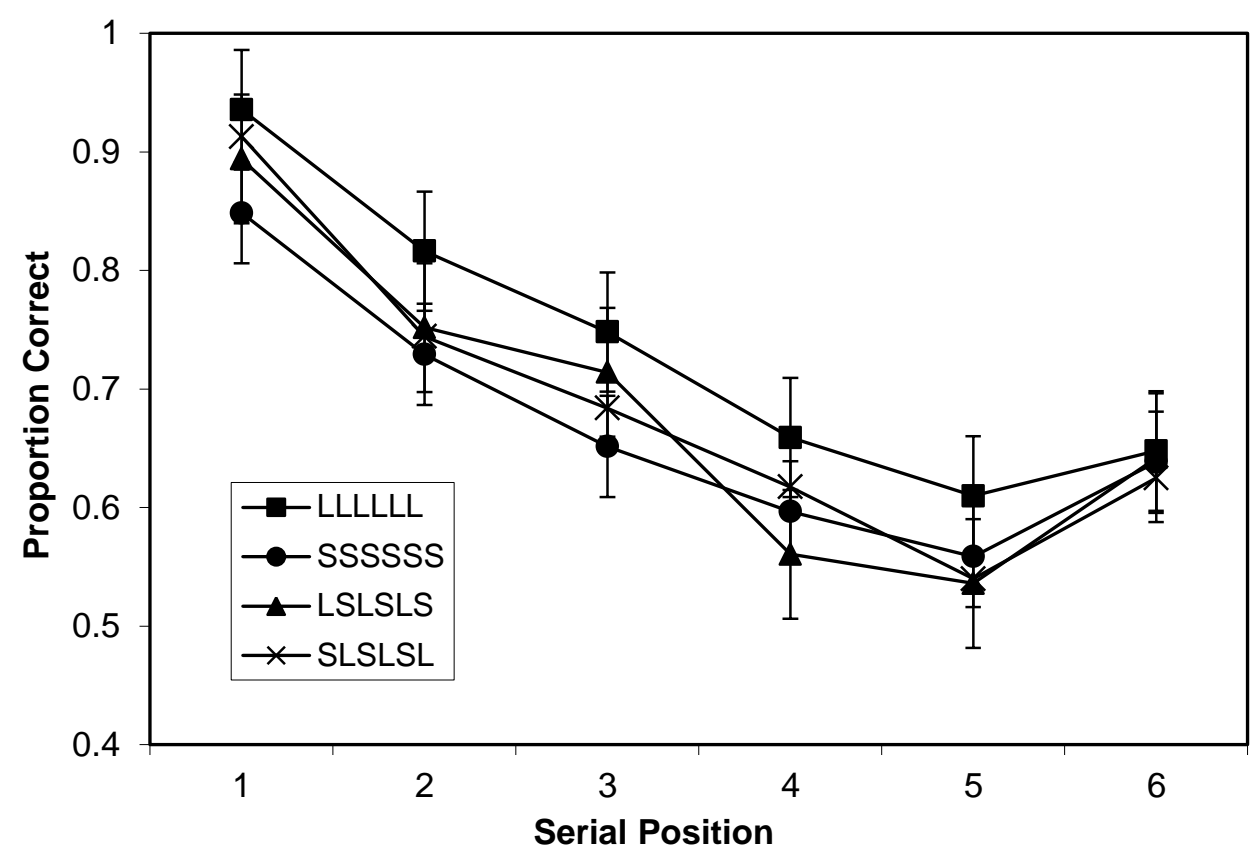

Figure 8.4. Serial order reconstruction performance across serial position as a function of neighbourhood size and list type. 
The serial position variable violated the sphericity assumption $(W(14)=.04, p$ $<.001)$. The resulting analysis for this effect was adjusted using the GreenhouseGeisser statistic. There was a significant effect of serial position, $F(2.40,50.34)=$ $38.40, p<.001$, reflecting the serial position curve depicted in Figure 8.4. This shows a decrement in performance after the first item until the last item.

The interaction between phonological neighbourhood density and condition was significant suggesting that the effect of phonological neighbourhood density was different for pure and mixed lists, $F(1,21)=5.24, p=.033$. An analysis of simple effects revealed that there was a significant neighbourhood effect for pure lists, $F$ (1, $21)=9.73, p<.01$, with better reconstruction performance for large neighbourhood lists $(M=.73, S E=.21)$ than for small neighbourhood lists $(M=.63, S E=.21)$. There was no significant difference between large $(M=.66, S D=.22)$ and small $(M=.65$, $S D$.23) neighbourhood sets in mixed lists, $F(1,21)=.10, p>.05$.

The interaction between list condition and serial position violated sphericity assumptions, $W(14)=.24, p=.017$. The resulting analysis for this interaction was adjusted using the Greenhouse-Geisser statistic. There was no significant interaction between list condition and serial position, $F(3.64,76.34)=1.46, p=.228$. This indicated that the two list composition conditions changed across serial position in the same way.

There was no significant interaction between phonological neighbourhood density and serial position, $F(5,105)=1.29, p=.274$. This means that large and small neighbourhood words changed across serial position in the same way.

Finally, the three way interaction between list composition, phonological neighbourhood size and serial position was not significant, $F(5,105)=.82, p=.538$. This indicated that the recall pattern of large and small neighbourhood words across serial position is the same for pure and mixed lists.

Further repeated measures analyses were conducted to ascertain whether mixed list performance is influenced by whether a large or a small neighbourhood word is presented first. A 2 (list configuration $L S L S L S$ vs. SLSLSL) by 6 (serial position) Repeated Measures ANOVA revealed no significant effect of list configuration, $F(1,21)=.06, p=.817$. This suggests that there is no difference in overall performance regardless of whether a large neighbourhood or a small neighbourhood word appears first. 


\subsubsection{Discussion}

There was no overall main effect of list composition. This is expected given that performance on pure lists is based on an average of the performance on small and large neighbourhood words, as is mixed lists. However, this differs from the betweensubjects analysis of the combination of Experiment 4 and Experiment 5 where there was a marginally significant effect, with a trend for performance in the mixed lists to be better than performance in the pure lists. This may be due to individual differences. Participants in the mixed list paradigm (Experiment 4) were recruited earlier in the university session than participants in the pure list experiment (Experiment 5), or the within-subjects experiment (Experiment 6), which may lead to quantifiable differences between the two participant cohorts.

There was still a significant effect of neighbourhood density in pure lists with overall better performance for large neighbourhood words than for small neighbourhood words. This is an important replication of the result from Experiment 5 given the novelty of the effect and its theoretical ramifications. As with Experiment 5, the significant neighbourhood effect indicates that phonological neighbourhood density does influence memory for order. Further, there was no effect of neighbourhood density in mixed lists, replicating results from Experiment 4.

Finally, the three way interaction between phonological neighbourhood density, list condition and serial position was not significant, replicating the result from the between-subjects analysis. It appears from Figure 8.4 that performance on mixed lists is equivalent to performance of small neighbourhood words in pure lists. This differs from the finding from the combined analysis of Experiment 4 and Experiment 5, where performance on mixed lists appeared to be equivalent to performance of large neighbourhood words in pure lists. Performance overall in Experiment 6 appeared to be worse than that of Experiment 4 and Experiment 5. Indeed, participants in Experiment 6 recalled an average of $69 \%$ of the words in each set while those in Experiment 4 and Experiment 5 combined recalled an average of $78 \%$ of the words in each set. This may be due to fatigue effects associated with an increase in number of trials or differences between the groups of participants in the different experiments. While this decrement in performance occurs for all conditions, it appears to have especially affected the mixed list condition. This result is 
unanticipated and difficult to explain, given that lists were counterbalanced, but is likely not to be of theoretical importance.

Finally, analyses were conducted to determine whether there is an effect of list configuration. That is, whether starting a list with a large neighbourhood word influences overall performance of the list compared to starting the list with a small neighbourhood word. It was possible that beginning a mixed list with a highly memorable large neighbourhood word would improve overall recall of this configuration of mixed lists. This was not supported in the current experiment with statistically equivalent performance for lists that began with a small neighbourhood word and those that began with a large neighbourhood word.

\subsection{General Discussion}

The results of the current phase of experiments confirm results from Allen and Hulme (2006) and suggest that phonological neighbourhood density does influence memory for order. The results are indicative of an influence of long-term memory on memory for the order of pure lists of large and small neighbourhood words, but no such influence on memory for order in mixed lists. If items were just coded with respect to their absolute serial position, then there is no apriori reason to expect that large neighbourhood words would lose their advantage over small neighbourhood words when presented in mixed lists, unless by doing so they acted to anchor small neighbourhood words. It appears from Figure 8.3, that this is the case. However, Figure 8.4 suggests that performance in mixed lists mirrors performance of small neighbourhood words in pure lists. This suggests that the inferior memory for small neighbourhood words may be negatively affecting performance on large neighbourhood words in mixed lists. This is reminiscent of predictions made by the order-encoding hypothesis, although this account would predict an intermediate level of performance for mixed lists compared to pure lists, which was not found in the current experiments.

Alternatively, the results could suggest a list-level effect, such as that suggested by the co-occurrence hypothesis. As discussed, according to this theory, cooccurrence exerts an influence over the whole list via a general level of supportive activation. Again, this would predict an intermediate level of recall for mixed lists, 
with the finding of mixed list performance equivalent to small neighbourhood words in pure lists presenting a challenge for this hypothesis. Further challenge for this hypothesis comes from Miller and Roodenrys (2012), who showed that the arrangement of high and low frequency words in mixed lists influences recall performance. The co-occurrence hypothesis would predict that how large and small items are arranged within the list would not influence overall performance as activation is available to all list items regardless of their position within the list.

Neither the order-encoding nor the co-occurrence accounts of memory for order are sufficient explanations of the effect of phonological neighbourhood density on memory for order. Given the finding of a phonological neighbourhood effect on memory for order is novel it is imperative that the conditions under which it appears are identified. A more in depth discussion and theoretical interpretation of the results will be given following the next phase of experiments. 


\section{Chapter Nine}

\section{The Phonological Neighbourhood Effect on Short-Term Memory for Order: Replicating the Effect}

\subsection{Introduction}

The experiments so far indicate that phonological neighbourhood density has a deleterious influence on memory for order. This is evidence for a long-term memory effect occurring prior to the redintegration of items. The finding that neighbourhood density only modulates memory for order in pure lists and not in mixed lists is evidence against purely positional models, such as Burgess and Hitch (1999), that code position independently of other list items. As discussed, it is possible that the superior order memory for large neighbourhood words may be constraining the movement of small neighbourhood words resulting in improved performance for small neighbourhood items in mixed lists, or may be an effect at the list-level such as that predicted by the co-occurrence hypothesis. However, these explanations would necessitate a performance level for mixed lists that occurs at an intermediate level to that observed between pure and mixed lists. It is evident from Experiment 6 that reconstruction performance on mixed lists is equivalent to that of than for pure lists of small neighbourhood words, a result that is more difficult to explain.

Before exploring this further a number of possible confounds have been identified that limit the interpretation of these data. The aim of the third phase of experiments was to replicate the phonological neighbourhood effect on memory for order, and to further explore the conditions under which it appears. The first experiment in this series, Experiment 7, was conducted to address the potential that the effect is due differences in processing time between large and small neighbourhood sets. While unlikely, it is necessary to clarify that this has not influenced the results. 
Chapter Nine: The Phonological Neighbourhood Effect

on Short-Term Memory for Order: Replicating the Effect

\subsection{Experiment 7: Investigating Difference in Reaction Time}

\subsubsection{Rationale}

The results of Experiment 6 were concordant with those from the amalgamation of Experiment 4 and Experiment 5, with a neighbourhood effect in reconstruction of pure list sets but not for mixed list sets. This effect is such that reconstruction performance was better for large neighbourhood lists than for small neighbourhood lists. Before investigating these conclusions further, potential confounds must be addressed to ensure accurate conclusions are made. One issue is the possibility that output interference influenced the results. There are two potential sources of output interference in the current experiments.

First, it is possible that differential processing speeds for large and small neighbourhood words may lead to output interference. The output stage of serial reconstruction of order involves participants reading items off a visual display unit and placing them back in their presented order. Due to this requirement to read the words it is possible that the neighbourhood effect observed could be due to differences in processing speed. That is, it could take an inequitable amount of time to read and recognise small neighbourhood words than it does to read and recognise large neighbourhood words. Yates (2005) investigated the effect of phonological neighbourhood density on processing written words using lexical decision, naming and semantic categorisation tasks. In all three tasks there was an advantage for large phonological neighbourhood words. Yates concluded that phonological neighbourhood density facilitates visual word recognition. This processing advantage afforded to large neighbourhood words may have contributed to the phonological neighbourhood effect by decreasing the amount of time taken to locate a large neighbourhood word during reconstruction. This processing time advantage could result in less decay of order information due to reduced output latency, resulting in better order memory for these items. In order to rule this out as a possible explanation reaction time can be taken during the output stage.

Second, output interference could occur due to differences in the time taken to write items down during recall. This may have occurred as there were inconsistencies in average word length between the large and small neighbourhood size stimuli sets. 
Specifically, words from large neighbourhood sets had on average fewer letters $(M=$ $3.96, S D=.59)$ than small neighbourhood sets $(M=4.53, S D=.83), t(46)=-3.88, p$ $<.001$. This should not affect the results from the first phase of experiments as these used auditory presentation and spoken recall, and all words followed a single syllabic CVC structure. However, in the second phase of experiments there may have been an influence of item length owing to the shift to visual presentation and written recall. This may have resulted in output interference. Evidence that output interference has the potential to influence results comes from a study by Cowan et al. (1992). They examined whether the effect of word length on serial recall was a result of output interference by presenting lists where the length of words varied across the two halves of the list. They found that recall was worse when long words were presented in the first half of the list. By varying forward and backward recall and post-cuing to ensure consistency with rehearsal, they found that performance was worse when the longer words were recalled first. This suggests that the word length effect is primarily due to output interference.

These issues were addressed in Experiment 7 by using a serial reconstruction of order task with the program adjusted to remove the requirement to write down words. Instead, participants were instructed to click the boxes on the screen in the order of presentation. Further, response latencies were recorded to determine whether a difference in the amount of time it takes to read large or small neighbourhood items off the screen was causing output interference.

\subsubsection{Hypotheses}

It was expected that the phonological neighbourhood effect observed in the pure lists presented in Experiment 6 would be replicated in Experiment 7. That is, it is expected that memory for the sequential order of lists of large neighbourhood words would be better than that of small neighbourhood lists.

With regards to findings on reaction time data, if the effect of phonological neighbourhood size is due to differences in processing speed between the two sets of stimuli then there should be differences in reaction times recorded during the output stage. Alternatively, if the results obtained in previous experiments showing an order 
advantage for large neighbourhood words is not due to differences in processing time then there should be no differences in reaction time data between the two list types.

\subsubsection{Method}

\subsubsection{Participants}

Thirty-three participants ( 9 male, 24 female) were recruited from the University of Wollongong School of Psychology subject pool. Participants were native English speakers and no participant who had participated in previous experiments could take part in Experiment 7. None of the participants reported visual or auditory impairments.

\subsubsection{Stimuli}

Stimuli were identical to those used in previous experiments.

\subsubsection{Procedure}

Stimuli were arranged into pure lists of large and small neighbourhood words with the stipulation that no word could appear more than once in each list. Twentyfour lists were created for each stimuli condition. Participants performed a serial reconstruction of order task in an individual testing session that lasted 20 minutes. List items were presented to participants through a set of headphones at a rate of 1 item per second. After all 6 items had been presented the words appeared vertically on a 20-inch VDU. Items appeared in 48-point Helvetica front as white text on a black background. The participants were asked to indicate the original presentation order by clicking on checkboxes that corresponded to each of the items. Once a participant had indicated a response they were unable to alter it. The program recorded the time lag between each checked item. Participants could proceed to the next list at their own pace. Lists were blocked by neighbourhood condition, and presentation of lists was counterbalanced between participants. The experimenter remained in the room throughout the experiment to ensure compliance with instructions. Following 
completion of all 48 lists, participants were debriefed on the goals of the study and were thanked for their participation.

\subsubsection{Results}

A 2 (phonological neighbourhood size) by 6 (serial position) Repeated Measures Analysis of Variance was conducted with list presentation order entered as a between subjects variable with two levels (large neighbourhood lists first v small neighbourhood lists first).

There was a marginally significant effect of phonological neighbourhood size, $F(1,34)=3.94, p=.055$. This effect is shown in Figure 9.1. There appears to be a fanning out of an effect after the fourth serial position, with a slight advantage for large phonological neighbourhood words after this point. While the large neighbourhood lists appear to have an advantage in the first three serial positions, this does not seem to be a significant advantage.

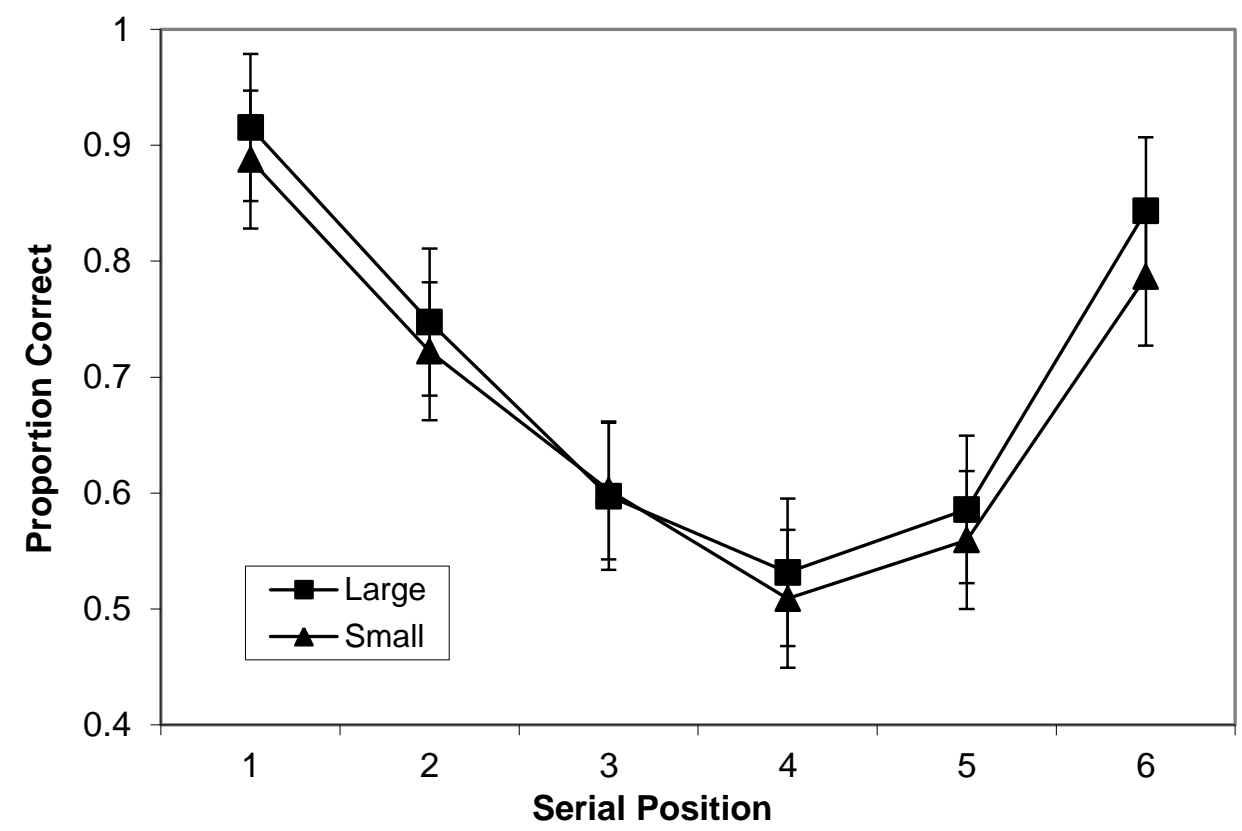

Figure 9.1 Mean proportions correct across serial position for large and small phonological neighbourhood words.

The effect of serial position was significant, $F(5,170)=103.10, p<.001$, reflecting the typical serial position curve found in previous experiments. There was no significant interaction between neighbourhood size and serial position $(F(5,170)$ 
$=.99, p=.425)$ indicating, despite appearances, that the marginally significant effect of neighbourhood size was consistent across serial positions.

The main effect of list presentation order (block) was marginally significant, $F$ $(1,34)=3.73, p=.062$. Performance was better for participants who received small neighbourhood lists first $(M=.73, S D=.20)$ compared with those who received large neighbourhood lists first $(M=.66, S D=.22)$.

As can be seen in Figure 9.2, there was no significant interaction between neighbourhood size and block, $F(1,34)=.87, p=.358$. This means that the main effect of neighbourhood size was the same for those who received the large neighbourhood block first as for those who received the small neighbourhood block first.

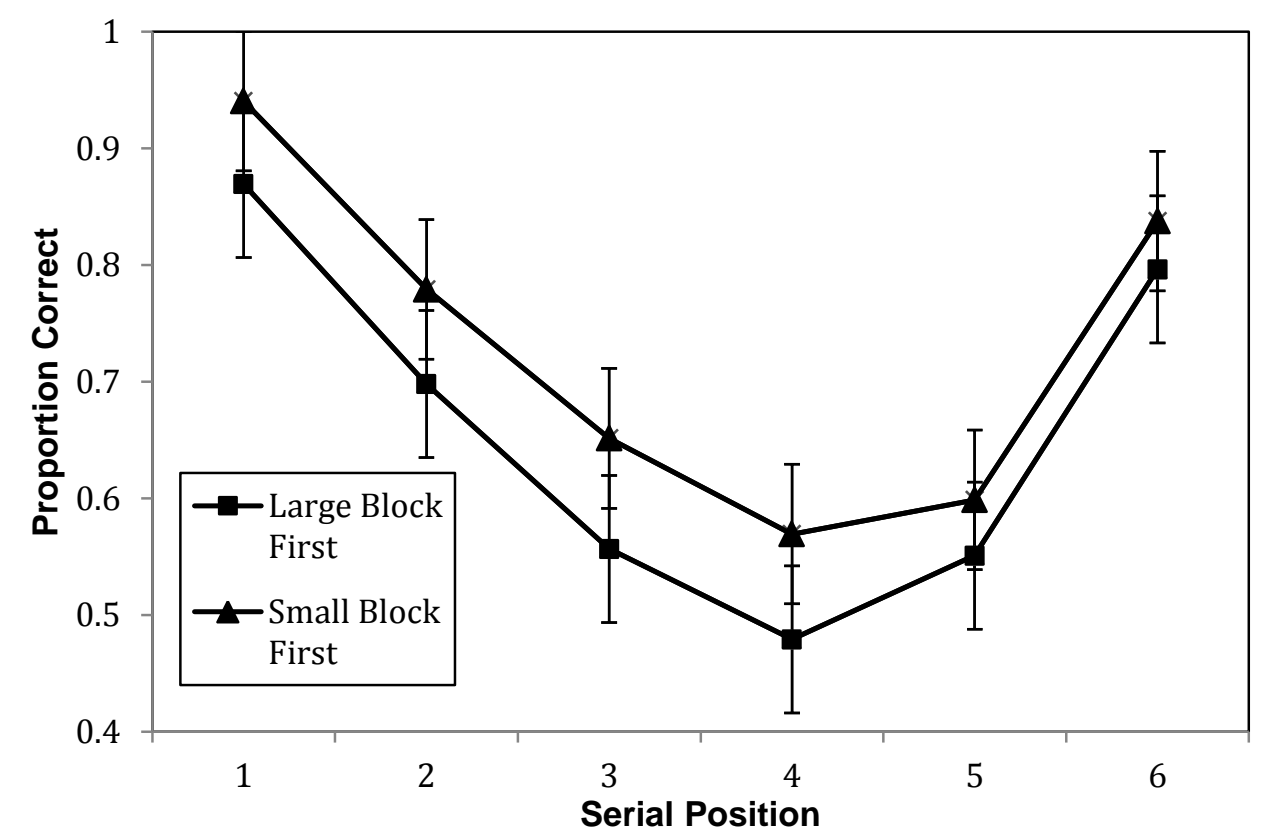

Figure 9.2 Serial reconstruction performance by block order (large neighbourhood words first or small neighbourhood words first) across serial position.

There was no significant interaction between block and serial position, $F$ (5, $170)=.93, p=.465$. This indicated that the serial position curve was the same for those who received small neighbourhood lists first as it was for those who received large neighbourhood lists first.

Finally, the three way interaction between block, phonological neighbourhood size, and serial position was not significant, $F(5,170)=.99, p=.424$. This means that the change across serial position for large and small neighbourhood lists was the 
same regardless of whether they received the small neighbourhood lists firsts, or whether they received the large neighbourhood lists first.

\section{Reaction Time}

Reaction time data (recorded in milliseconds) was collected for each response across serial position. Reaction time data were converted to reflect the time elapsed between the current item recalled and the previous item recalled. For the item in the first serial position the response time reflected the time elapsed since the reappearance of the list items and the first button press. The data were averaged across the number of trials for each condition for each participant and were subject to a 2 (neighbourhood size) by 6 (serial position) Repeated Measures ANOVA.

There was no significant effect of neighbourhood size, $F(1,33)=.36, p=$ .554. This indicated that there was no overall difference between the amount of time taken to select large neighbourhood words and the amount of time taken to select small neighbourhood words.

There was a significant effect of serial position, $F(5,165)=23.07, p<.001$. This is depicted in Figure 9.3 and reflects a decrease in reaction time across serial position as the number of possible items decreases.

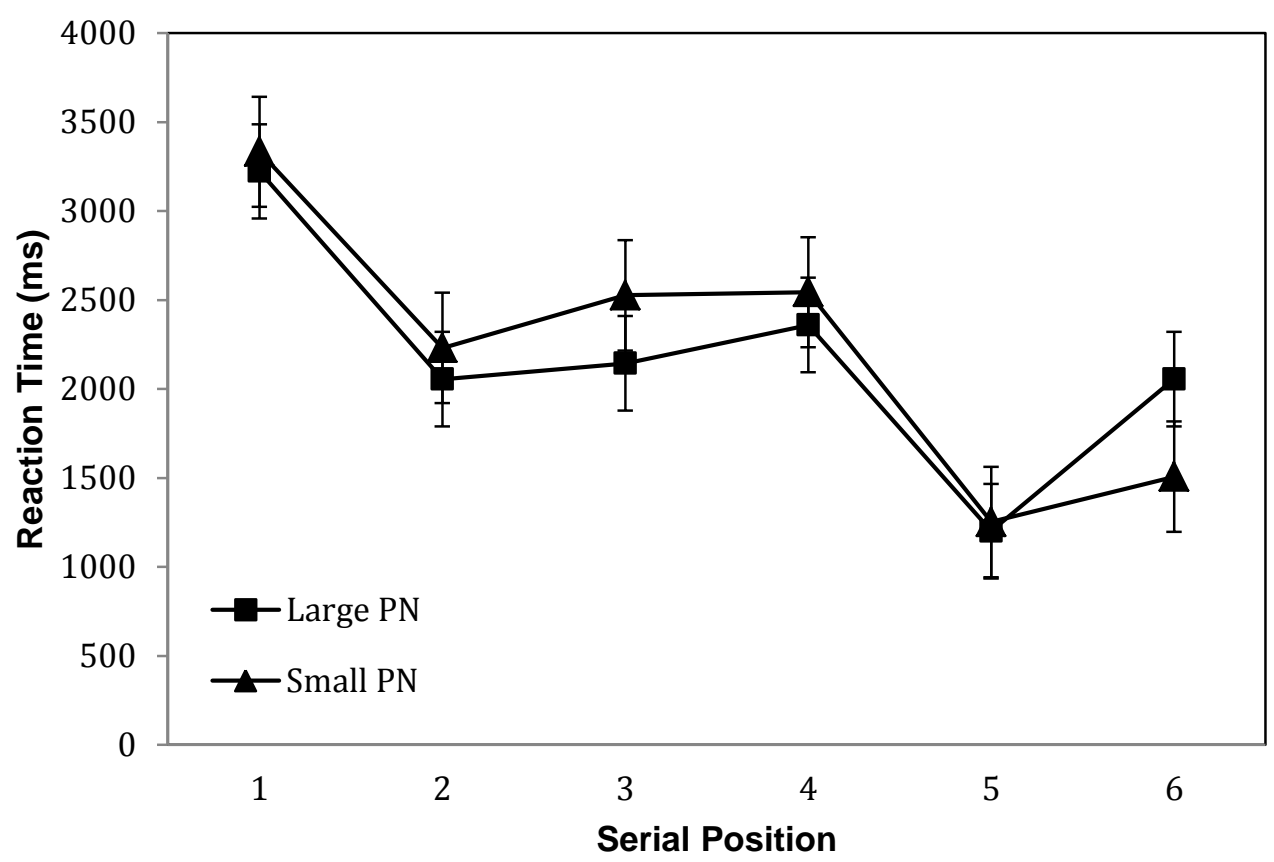

Figure 9.3 Reaction time in milliseconds across serial position for large and small neighbourhood words. 
Chapter Nine: The Phonological Neighbourhood Effect

on Short-Term Memory for Order: Replicating the Effect

There was no significant interaction between neighbourhood size and serial position, $F(5,165)=1.20, p=.312$. This indicated that change across serial position was the same for small and large neighbourhood words.

\subsubsection{Discussion}

There was a marginally significant effect of phonological neighbourhood density in Experiment 7. The direction of the effect was in line with previous experiments, with superior reconstruction performance for lists of large neighbourhood words compared with small neighbourhood words. This effect may have failed to reach significance because of error variance introduced by the marginally significant effect of block. The effect of block is such that reconstruction performance was better overall for participants who receive small neighbourhood lists first, compared with those who receive large neighbourhood lists first. As list block was counterbalanced between participants it is improbable that this result reflects something to do with a specific cohort. Instead, the effect may reflect strategic differences in approaching serial reconstruction based on whether participants were presented with large neighbourhood lists first, or small neighbourhood lists first. As large neighbourhood lists are easier to remember, participants may not have needed to develop mnemonic strategies as basic rehearsal was sufficient for a reasonable level of recall. Once the lists changed to the more difficult small neighbourhood words, participants' well-versed rehearsal strategies that were sufficient for large neighbourhood lists may have become ineffectual. If small neighbourhood lists were presented first, participants may have been required to develop mnemonic strategies such as grouping, forging semantic links between items, and so on, as these items are inherently more difficult to remember. By the time the large neighbourhood lists were presented, participants may have developed effective approaches to encoding and maintenance of the items that would continue to assist the recall of the large neighbourhood items.

It is also possible that the effect found in previous experiments was, in part, due to differences in word length between stimuli and the neighbourhood effect is attenuated here because the confound is removed. The finding of a marginal difference between the two list types in this experiment is still evidence that 
neighbourhood density is playing some role, albeit a small one, in the order memory of these stimuli. This said, further experiments need to be conducted to ascertain whether neighbourhood density has a significant effect on order memory after the elimination of the word length confound, which may indicate that the failure of an effect here was indeed due to a confound with block.

There was no significant effect of neighbourhood density on reaction time indicating that the neighbourhood effect is not due to differences in processing written words during output. It is evident from this and previous experiments that a small effect of phonological neighbourhood density on short-term memory for order exists. It is unclear from the current experiments where the locus of this effect lies, although it is evident that it is not due to differential processing time between large and small neighbourhood words or due to a word length effect. The phonological neighbourhood effect in Roodenrys et al. (2002) experiments was attributed to processes operating during speech production. Roodenrys and his colleagues did not elucidate whether the neighbourhood influence on item memory or on order memory was attributable to the speech production system. The following experiments examine the thesis that the locus of the neighbourhood effect on memory for order lies in the speech production system.

\subsection{Experiment 8: Removing Speech Production using Articulatory Suppression}

\subsubsection{Rationale}

Theoretical models are divided with regards to whether speech production processes are responsible for item memory or order memory or both. With regards to non-psycholinguistic models, a common conception is that verbal long-term memory provides a basis for remembering item information while order information is processed and stored by a separate dedicated system that is independent of the language system (Majerus \& D’Argembeau, 2011). Further, psycholinguistic models where activation is strictly feed-forward would also predict that serial order is independent of articulatory processes (i.e. Levelt et al., 1999). This is because articulatory processes occur at the end of the process after the order of sequences has 
been determined. With no bidirectional links, these feed-forward models lack the feedback needed for articulatory processes to influence serial order.

The experiments conducted so far are neutral with respect to conclusions made about the influence and role of speech production processes. While the second phase of experiments did not require participants to employ vocal articulation, they may have used subvocal articulation to aid memory of the sequential order. Experiment 8 aimed to test whether speech production processes are the locus of the effects of neighbourhood density on memory for order by requiring participants to engage in articulatory suppression during the presentation of the items and while reconstructing the list. Articulatory suppression involves repeating an irrelevant word to prevent participants from using speech production processes to rehearse items (Murray, 1968). An influence of articulatory suppression can be taken as evidence that the short-term memory system uses processes involved in speech production (Camos et al., 2009). If the phonological neighbourhood effect on memory for order disappears under the influence of articulatory suppression, then the postulation of independent item and order processes loses credence. Instead, if the phonological neighbourhood effect disappears when articulation is suppressed, then this indicates that as well as processing item memory, verbal long-term memory also influences the storage and retrieval of order memory. This rationale follows that of Saint-Aubin and Poirier (1999a), who used articulatory suppression with a serial reconstruction of order task to examine semantic similarity effects on order information. They found an overall reduction in performance under conditions of articulatory suppression comparative to the effect found in serial recall.

\subsubsection{Hypotheses}

If the phonological neighbourhood effect found in previous experiments was due to vocal or subvocal articulation of the list during the trials, then it was expected that the effect would disappear under conditions of articulatory suppression. If the effect was due to short-term memory processes that do not rely on articulation, then it was expected that effect would remain when participants are asked to vocalise an irrelevant word. 


\subsubsection{Method}

\subsubsection{Participants}

Eighteen participants were involved in Experiment 8 ( 7 males and 11 females). Participants were recruited from the University of Wollongong School of Psychology subject pool. Participation was limited to native English speakers who had not participated in Experiment 7. Participants did not report and problems with hearing or understanding the stimuli.

\subsubsection{Stimuli}

Stimuli were identical to those used in previous experiments.

\subsubsection{Procedure}

Large and small phonological neighbourhood words were used to create 24 lists of six words for each neighbourhood condition, giving a total of 48 lists per participant. Words were randomly allocated into pure lists of large and small neighbourhood words with the restriction that words could not appear more than once in the same list. Large neighbourhood lists and small neighbourhood lists were presented in their own 24 list series. The order of presentation (large neighbourhood block first or small neighbourhood block first) was counterbalanced across participants. Participants were individually tested in a single 30 -minute session. The procedure followed the serial reconstruction procedure used in Experiment 7 with one exception; in the current experiment participants were required to repeat the word 'coca-cola' while listening to words and also while they were selecting their responses. The experimenter remained in the room throughout the experiment to ensure adherence to these instructions. Upon completion of the experiment, the participants were debriefed and were thanked for their participation. 
Chapter Nine: The Phonological Neighbourhood Effect on Short-Term Memory for Order: Replicating the Effect

\subsubsection{Results}

Items placed in the correct serial position were analysed using a 2 (neighbourhood size) by 6 (serial position) Repeated Measures Analysis of Variance.

There was no significant effect of phonological neighbourhood density on reconstruction performance under conditions of articulatory suppression, $F(1,17)=$ $.02, p=.893$.

The effect of serial position was significant, $F(5,85)=64.81, p<.001$. This is reflected in Figure 9.4, which shows a typically serial position curve with better performance for the first and last items in the list.

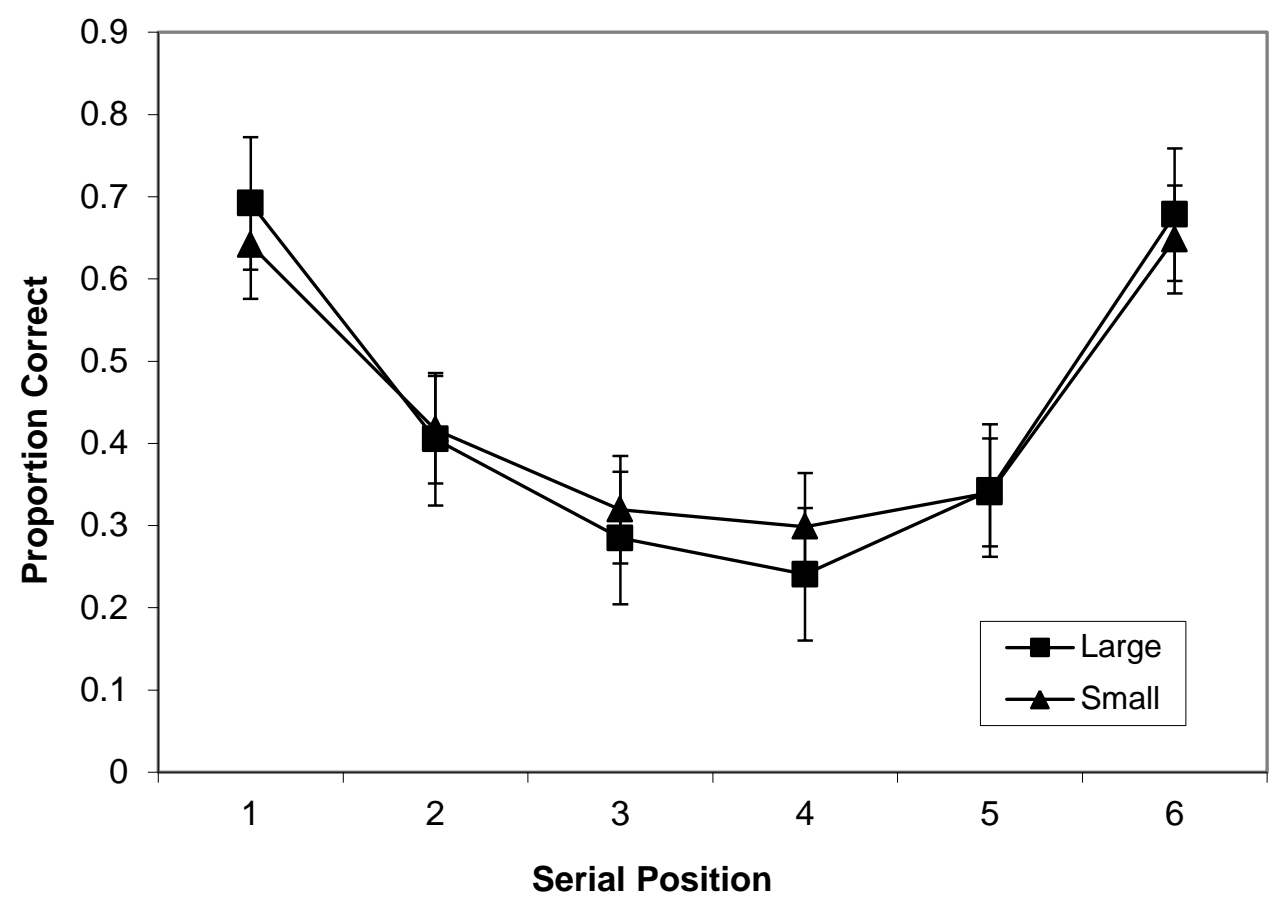

Figure 9.4 Mean proportion of items placed in the correct position for large and small neighbourhood sets.

There was no interaction between serial position and phonological neighbourhood size, $F(5,85)=1.93, p=.099$. This indicated that the pattern of performance across serial positions was the same for large neighbourhood lists as it was for small neighbourhood lists. 
Chapter Nine: The Phonological Neighbourhood Effect on Short-Term Memory for Order: Replicating the Effect

\subsubsection{Discussion}

Experiment 8 was conducted to address the role of articulatory processes in short-term memory for order. It was expected that if the phonological neighbourhood effect on memory for order observed in previous experiments was due to influences operating in the speech output path, then the neighbourhood effect would disappear when articulation of the items was discouraged by using articulatory suppression. This was confirmed in Experiment 8, with no effect of neighbourhood density on serial reconstruction performance when participants were required to articulate an irrelevant word. It is important to note that while articulatory suppression eliminated the effect of neighbourhood density, it did not completely decimate performance. While there was a drop in performance level between this experiment and previous experiments not requiring articulatory suppression, this can be attributed to an increase in cognitive load. This is consistent with a decrement in performance found in other experiments using articulatory suppression and serial reconstruction (SaintAubin \& Poirier, 1999a).

Before any further interpretation of these results it is necessary to rule out a possible confound. Although unlikely, it is possible that the inability to find an effect of neighbourhood density is, in part, due to error variance associated with blocking rather than an effect of concurrent articulation. This was suggested as a potential explanation for the finding of only a marginally significant effect of neighbourhood density in Experiment 7. To examine whether the effect of block contributed to the non-significant neighbourhood effect in Experiment 8, small and large neighbourhood lists can be randomly presented rather than presented in blocks.

\subsection{Experiment 9: Removing the Influence of Blocking: Serial Reconstruction of Order with Articulatory Suppression}

\subsubsection{Rationale}

Experiment 8 revealed that the significant effect of neighbourhood density obtained in previous experiments was eliminated when concurrent articulation was required. This suggests that the locus of the neighbourhood effect on short-term 
memory for order lies within the speech production system. Before exploring this further, it is necessary to determine whether the lack of neighbourhood effect in Experiment 8 was inaccurately attributed to the introduction of articulatory suppression and is instead due to error variance associated with blocking large and small neighbourhood lists together. This was identified as a possible explanation for the marginalisation of the neighbourhood effect in Experiment 7 where performance was better for trials where lists of small neighbourhood words were presented first. Experiment 9 aimed to investigate this by following the paradigm of Experiment 8 with one difference. Instead of presenting large neighbourhood lists and small neighbourhood lists in blocks, they will be randomly presented to participants. Further, the word participants were required to articulate was changed from 'cocacola' to 'elephant' to ensure that any effect of articulatory suppression was not specific to the word used. It was observed that the repetition of 'coca-cola' in Experiment 8 caused some participants to become tongue-tied. This may have resulted in participants allocating excessive attentional resources to eliciting the word coca-cola, which may have detracted from the actual memory task.

\subsubsection{Hypotheses}

It was expected that if the phonological neighbourhood effect is due to speech production processes then there would be no effect of neighbourhood size on reconstruction performance when concurrent articulation is required.

\subsubsection{Method}

\subsubsection{Participants}

Twenty-six participants (20 female, 6 male) were recruited from the University of Wollongong's School of Psychology subject pool. Participants were all undergraduate psychology students who participated in return for course credit. Participants all had English as their native language and none reported any sensory difficulties that would interfere with completion of the experiment. 
Chapter Nine: The Phonological Neighbourhood Effect on Short-Term Memory for Order: Replicating the Effect

\subsubsection{Stimuli}

Stimuli were identical to those used in previous experiments.

\subsubsection{Procedure}

Stimuli were pseudorandomly arranged into lists of 6 words within each neighbourhood condition. There were 24 lists of large neighbourhood words and 24 lists of small neighbourhood words, giving a total of 48 lists for each participant. Stimuli were arranged such that no two words could appear in the same list but words could appear on subsequent lists with no constraints. Following creation of 24 lists for each condition, the lists were randomised so that lists containing large neighbourhood words would appear randomly among lists containing small neighbourhood words.

Participants were individually tested using a serial reconstruction of order task in a single session that lasted 30 minutes. The task protocol followed that of Experiment 8 with one exception; rather than repeating the word 'coca-cola' aloud throughout the experiment participants were required to repeat the word "elephant". Once again, participants were required to maintain the repetition of the word 'elephant' throughout presentation and recall. Once participants had indicated the order of all 6 items, they could proceed to the next trial via a button click.

Participants received four practice trials followed by the 48 experimental trials. Following completion of all trials, participants were debriefed and were thanked for their participation. The experimenter remained in the room throughout the experiment to ensure compliance with these instructions.

\subsubsection{Results}

An item was scored as correct if it was the correct item in the correct serial position. Data were analysed using a 2 (phonological neighbourhood size) by 6 (serial position) Repeated Measures Analysis of Variance. Figure 9.5 shows the mean proportion correct across serial position on the serial reconstruction of order task. There was no significant effect of phonological neighbourhood density, $F(1,25)=$ $.41, p=.530$. This indicated that, under conditions of articulatory suppression, 
reconstruction performance does not differ for large and small phonological neighbourhood words.

There was a significant effect of serial position, $F(5,125)=64.13, p<.001$. This reflects the recency and primacy effects found in the literature and in previous experiments.

Finally, there was no significant interaction between phonological neighbourhood size and serial position, $F(5,125)=.63, p=.678$. This means that small neighbourhood and large neighbourhood lists changed across serial position in the same way.

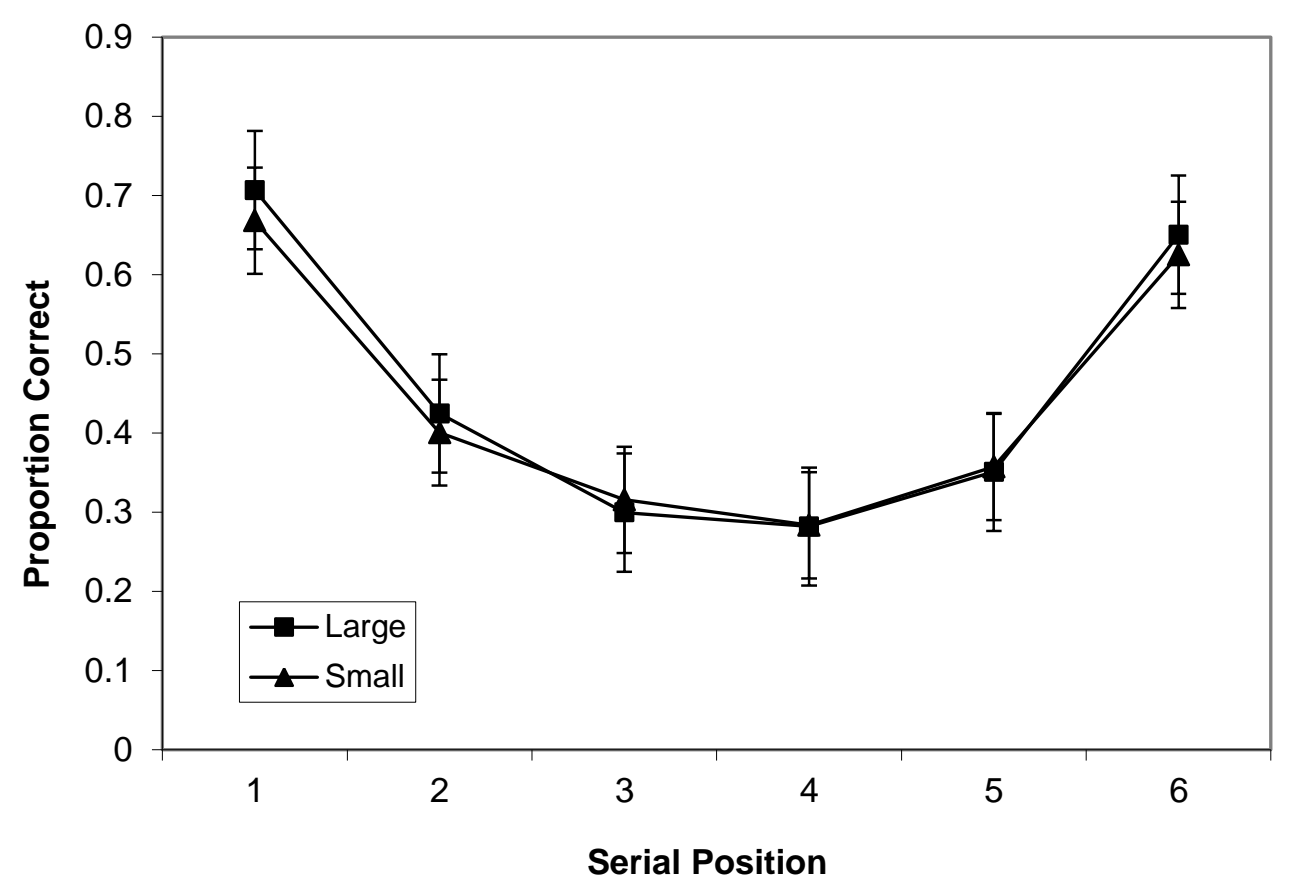

Figure 9.5 Serial reconstruction performance by block order (large neighbourhood words first or small neighbourhood words first) across serial position.

\subsubsection{Discussion}

It was expected that if the phonological neighbourhood effect observed on memory for order was due to processes operating during speech output then there should be no effect of neighbourhood density when output processes are interrupted by articulatory suppression. This finding was supported in Experiment 9, with no effect of neighbourhood density at any serial position in a serial reconstruction task. 
Chapter Nine: The Phonological Neighbourhood Effect on Short-Term Memory for Order: Replicating the Effect

These findings confirm those from Experiment 8 and indicate that the phonological neighbourhood effect on memory for order is dependent on speech production processes. Experiment 9 rules out the possibility that the findings from Experiment 8 were due to an increase in error variance associated with an influence of block.

This finding highlights the importance of the speech output pathway in shortterm memory for order. The role of the pathway in short-term memory had been suggested previously. Martin, Lesch, and Bartha (1999) for instance investigated the serial recall of an anomic patient who had intact processing, comprehension and matching span. They found the patient had impairments in both naming tasks and serial recall, suggesting that serial recall is reliant on speech output processes. It was not clear from this case study whether speech output processes influenced item memory or order memory, as serial recall necessarily involves both. The current experiment indicates that these speech production processes exert an influence on memory for order specifically. This refutes earlier suggestions that verbal long-term memory only comes into play once the order of items has been retrieved. This is in conflict with approaches that argue for independent speech production and serial ordering processes, and goes against strictly feed-forward psycholinguistic models. Despite the disparity with such theories, a confluence between speech production and serial order is perhaps intuitive given the serial nature of speech production (Lashley, 1951).

While it is evident from current results that the locus of the phonological neighbourhood effect on memory for order is the speech production system it is unclear whether this occurs during maintenance of the items or during output. According to many models of short-term memory for order ascribing to the tracedecay plus rehearsal view, the speech production buffer is not engaged until the point of recall. Woodward, Macken, and Jones (2008) hold an alternate view of the mechanism of rehearsal in the trace decay plus rehearsal theory. Instead of viewing rehearsal as cyclical reactivation of a temporary phonological trace in order to offset decay, rehearsal is the cyclical implementation of the speech output plan relating to the whole sequence. That is, rehearsal necessarily involves speech output processes. Woodward and colleagues aimed to show that linguistic familiarity effects (including word frequency and lexicality) were at least in part due to the fluency of coarticulation rather than solely due to redintegration. Over four experiments they found 
Chapter Nine: The Phonological Neighbourhood Effect on Short-Term Memory for Order: Replicating the Effect

that increasing the familiarity of the co-articulation of items increased recall performance of lists, and that this increase in performance was not due to inter-item associations and was not an item level effect. This finding follows Geffen and Luszcz (1983), who found that it was not the variation in articulation between high and low frequency words that produced the word frequency effect, but the greater pauses between articulating uncommon words than common words.

While the current study is investigating phonological neighbourhood effects with unfamiliar lists, the idea that items with common sound sequences can be repeated faster than those with uncommon sound sequences has relevance for theoretical interpretation of these data. Perhaps words from large phonological neighbourhoods can be assembled in a sequence with greater ease than can small phonological neighbourhood words that typically have uncommon sound sequences. Rehearsal would be more successful for such lists as the reactivation of the speech output pathway operates on a sequence basis rather than on an item by item basis. As large phonological neighbourhood words have more common sound sequences the reactivation of this pathway would be faster and more successful than for small phonological neighbourhood words. The neighbourhood effect may have been present in previous experiments and not in the current experiments because the cyclical reactivation of the speech output pathway was not disrupted in prior experiments, but was disrupted once articulatory suppression was introduced. Miller and Roodenrys (2012) challenged the co-articulation hypothesis by showing that the word frequency effect persisted under conditions of articulatory suppression in ISR. It is possible that articulatory suppression specifically affects order information. Miller and Roodenrys (2012) used an ISR task that relies much more heavily on item memory, while the current experiment used an order reconstruction task. This may have been more sensitive to effects of articulatory suppression if suppression influences maintenance of order information more than item information.

Further evidence that these effects are occurring during rehearsal comes from Murdock and vom Saal (1967). They investigated whether transpositions occur during storage or retrieval of the items. They presented lists of three words to participants, with lists either semantically similar or dissimilar. They found that the same category lists were subject to a greater number of transpositions. Furthermore, by varying the retention interval they were able to determine whether the errors occurred during the 
maintenance or retrieval of information. If order errors were due to an influence on the retrieval mechanism then the proportion of order errors should not change over increased retention intervals. However, if order errors occur during the storage of information then there should be more order errors for the longer retention intervals. Murdock and vom Saal found evidence for the latter, with the proportion of order errors increasing over longer retention intervals.

It is important to point out that the interpretation of these results is being conducted in the absence of a control group within the study. A control group was not sought due to difficulties in obtaining sufficient participants. It is assumed that the cohort used in the articulatory suppression experiments does not differ from those who participate in earlier experiments and therefore results from earlier experiments are used as a comparison. Indeed the markers associated with serial recall, such as bowed serial position curves are evident in these two experiments indicating that the results are typical.

One possibility remains that limits the clear interpretation that long-term memory influences short-term memory for order. It is possible that serial reconstruction is not analogous to serial recall, and that the tasks are not measuring the same underlying short-term memory processes. Experiment 10 explores this possibility further.

\subsection{Experiment 10: Immediate Serial Recall and Serial Reconstruction of Order: Post-Cued.}

\subsubsection{Rationale}

Short-term memory involves the ability to encode information, maintain it over a short period of time, either by a rehearsal mechanism or through the intrinsic storage capacity of the store, and retrieve and output the information (Walker \& Hulme, 1999). ISR taps into these three processes that underlie short-term memory. The studies so far have been conducted on the assumption that ISR and serial reconstruction of order engage common processes for encoding and maintaining information in its serial order. The two tasks diverge, theoretically, on their reliance on redintegrative processes operating at the point of recall. In ISR, these processes are 
Chapter Nine: The Phonological Neighbourhood Effect on Short-Term Memory for Order: Replicating the Effect

necessary to reconstruct degraded traces. In reconstruction of order, redintegrative processes are unnecessary as items are presented at recall. To form a cogent argument that long-term memory mediates short-term memory for order, it is necessary to test this underlying assumption and demonstrate that ISR and serial reconstruction indeed use common processes. It could be that differing processes are used, meaning that results from the reconstruction task cannot be compared with those obtained using serial recall.

To test this, an experiment was devised which employs both ISR and serial reconstruction of order, with the type of task post-cued. Duncan and Murdock (2000) noted that post-cuing a task allows for control, to a degree, over how participants encode items. Given that serial recall is the more difficult of the tasks, it is expected that participants will prepare for every trial as if it is serial recall. In doing this, we will be able to ascertain whether participants are using the same or different processes in serial reconstruction of order as they are with serial recall.

\subsubsection{Hypotheses}

If participants are using the same processes as they use with serial recall all the time with serial reconstruction then the results obtained in Experiment 10 would mimic that of other experiments. That is, it was expected that there would be an advantage for lists of large neighbourhood words. Alternatively, if participants engage in different processes when performing a reconstruction of order task compared with ISR, then having them prepare for ISR and then giving them a reconstruction trial may yield different results to those obtained in previous experiments.

\subsubsection{Method}

\subsubsection{Participants}

Participants were 38 students (30 female, 8 male) from the University of Wollongong, who participated in the experiment in return for course credit. Participants were all native English speakers and none reported any auditory 
Chapter Nine: The Phonological Neighbourhood Effect

on Short-Term Memory for Order: Replicating the Effect

problems. Participants all had normal or corrected to normal vision. Participants were excluded if they had participated in previous experiments.

\subsubsection{Stimuli}

Stimuli were identical to those used in previous experiments.

\subsubsection{Procedure}

Stimuli were arranged into lists of 6 words within each neighbourhood condition, such that no word could appear twice within each list, but items could appear, without constraint, on subsequent lists. There were 32 lists of large neighbourhood words, and 32 lists of small neighbourhood words, giving a total of 64 lists. Each list was randomly determined a serial recall trial or a serial reconstruction trial to prevent participants from anticipating the task type. There were 32 reconstruction trials and 32 recall trials. Sixteen lists of each trial type were large neighbourhood lists and 16 were small neighbourhood lists. Large and small neighbourhood lists were randomly intermixed with one another. Participants were tested in individual sessions lasting 40 minutes. Items were presented to participants at a rate of 1 item per second, through headphones connected to a Macintosh computer. Following presentation of all 6 items, participants were cued to perform either a serial recall task or a serial reconstruction task. The cue for a serial recall task was a short beep. The cue for a serial reconstruction task was the words appearing on the 20-inch VDU vertically arranged in a random order in 48-point Helvetica font as white text on a black background. If participants were cued with a beep, they were required to recall the items out loud, starting from the first word that they heard and working their way through the list. Participants were advised to say "pass" for any words they would not remember. If participants were cued with the list items, they were required to put them back in the order they were presented by clicking on the checkboxes that corresponded to each of the items in order. Participants were unable to change a response once it was made. The experimenter remained in the room throughout the experiment to record the recall responses and to ensure compliance with instructions. Once participants had completed the experiment they were debriefed and thanked for their participation. 
Chapter Nine: The Phonological Neighbourhood Effect on Short-Term Memory for Order: Replicating the Effect

\subsubsection{Results}

In both the serial recall task and serial reconstruction task, an item was scored as correct if it was the correct item in the correct serial position. Data were analysed using a 2 (phonological neighbourhood size) by 2 (task type) by 6 (serial position) Repeated Measures Analysis of Variance. Figure 9.5 shows performance on the reconstruction task and the recall task across serial position for lists of large and small phonological neighbourhood words. Means are expressed as proportions of items recalled in each serial position.

There was a significant main effect of list type $F(1,37)=340.06, p<.001$, reflecting significantly better performance for the serial reconstruction task $(M=.65$, $S E=.24)$ than for the serial recall task $(M=.54, S E=.30)$.

The main effect of phonological neighbourhood size was significant, $F(1,37)$ $=5.53, p=.024$. Overall there was better performance for lists of large neighbourhood words $(M=.55, S E=.29)$, than lists of small neighbourhood words $(M=.52, S E=.29)$ regardless of task type.

There was also a significant main effect of serial position, $F(5,185)=153.05$, $p<.001$. This reflects the typical serial position curve typically found in short-term memory experiments.

There was no significant interaction between task type and phonological neighbourhood size, $F(1,37)=.04, p=.840$. This indicated that the phonological neighbourhood effect was the same in both the serial recall task and the serial reconstruction task.

There was a significant interaction between serial position and task type ( $F$ ( 5 , $185)=36.17, p<.001$ ), indicating that the effect of serial position differs between the serial recall task and the serial reconstruction task. Figure 9.6 shows this interaction. There is a greater decline in the serial position curve for the serial recall task than for the serial reconstruction task. The curve for the serial reconstruction task, while bowed, does not show such a sharp decrease in performance for the central items. This is simply an artefact of task demands with the curve for serial recall reflecting erroneous item recall as well as order errors, whereas the curve for the serial reconstruction task reflects order errors only. 


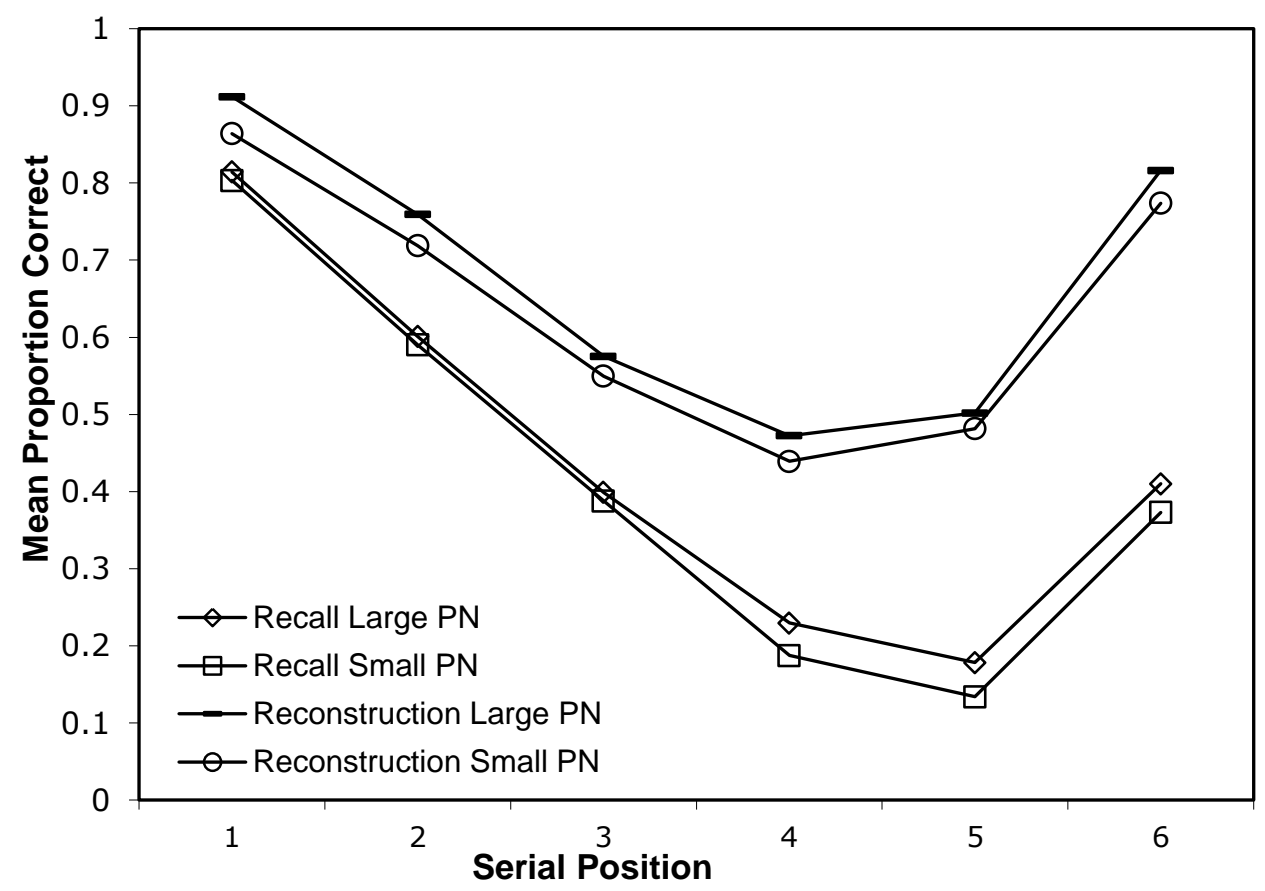

Figure 9.6 Serial reconstruction and serial recall performance for large and small neighbourhood lists.

There was no significant interaction between phonological neighbourhood size and serial position, $F(5,185),=.36, p=.873$. The change across serial position was the same for large and for small phonological neighbourhood words.

There was no three way interaction between task type, phonological neighbourhood density and serial position, $F(5,185)=.67, p=.646$, meaning that the consistent change across serial position for large and small neighbourhood words was the same for the serial recall task as it was for the serial reconstruction task.

\subsubsection{Discussion}

The aim of Experiment 10 was to investigate whether participants employ common processes when performing serial recall and serial reconstruction.

Performance was significantly better in the serial reconstruction task than in the serial recall task. This reflects the reconstruction task being easier than the recall task, as expected. This is because participants do not have to reconstruct the items at recall, as performance can still be successful even if participants can only remember one or two 
Chapter Nine: The Phonological Neighbourhood Effect on Short-Term Memory for Order: Replicating the Effect

phonemes in each word. Participants can also use strategies like the process of elimination if they have forgotten all phonemes of a word, so long as they only forget one word per list.

As the tasks were post-cued it is expected that participants will still encode and try to remember the entire word, and prepare for each trial as if it was a recall trial, as there is a 50 per cent chance the task will be a recall task. This was uncertain in previous reconstruction experiments as participants may have attempted to reduce the memory load by only focussing on maintaining parts of words. As performance on the immediate serial recall trials followed the same bowed serial position pattern expected for such trials, it is evident that participants did prepare for at least most trials as they would for serial recall. Indeed the lack of neighbourhood effect in the first few serial positions follows that found in Experiment 3. However, in this experiment the effect cannot be attributed to a ceiling effect as it was in Experiment 3. This is due to the overall lower performance in the current experiment. This may be due to additional task demands required from post-cuing recall and reconstruction. Alternatively, it may reflect specific strategies employed by participants to limit cognitive effort. As recognition and recall trials were presented randomly, participants may have anticipated a serial reconstruction trial if, by chance, they had a number of recall trials in a row.

One possible explanation for the fanning out of the phonological neighbourhood effect at the latter serial positions comes from a study by Martin and Saffran (1997) who noted that different levels of linguistic processing differ in their influence of items across a string. They note evidence that word frequency affects recall of items in the first serial positions (Watkins \& Watkins, 1977) while phonological similarity plays a role in the terminal portion of the series (Brooks \& Watkins, 1990). Martin and Saffran (1997) supported this claim with a study of 15 aphasic individuals. They found that primacy was linked with an ability to maintain semantic representations while phonological processing was associated with the recency portion. Martin and Saffran (1997) note that earlier items benefit from being in short-term memory for longer, as this gives them more opportunity to be rehearsed. This enables semantic representations in long-term memory to be activated. Recently presented items do not benefit from these processes and so are simply affected by phonological factors. This approach assumes that an item's representation is activated 
at all levels of the lexical system and maintains activation until a response occurs, which is in contrast with the phonological loop model, which assumes that the representations are purely phonological.

\subsection{Conclusion}

Results from the second phase of experiments confirm an effect of phonological neighbourhood density on memory for order. The studies conducted have not only replicated the neighbourhood effect but have narrowed the scope of possible explanations. First, the effect is not due to differences in processing large and small neighbourhood sets, either due to word length or reaction time. Second, the neighbourhood effect is likely to be occurring due to processes in speech production, as the effect disappears when concurrent articulation is required. This suggests that maintaining items in order makes use of speech production pathways. Third, the effect is not due to an influence of presentation order or the blocking of lists of the same neighbourhood size as the effect remains when large and small neighbourhood lists are presented in a random order; and continues to be absent under conditions of articulatory suppression when lists are presented randomly rather than blocked. Finally, the effect is not due to task specific processes occurring in serial reconstruction as the effect remains when participants prepare for recall trials but are instead given reconstruction trials. While this series of experiments confirms the neighbourhood effect on memory for order it is unclear whether this effect is generalizable to other long-term memory variables. This possibility will be examined in Chapter 10. 


\section{Chapter Ten}

\section{The Word Frequency Effect on Short-Term \\ Memory for Order}

\subsection{Introduction}

The results so far suggest that phonological neighbourhood density does influence memory for order. This effect cannot be explained by differences in word length or reaction time, and is not due to the order of trials. Further, the phonological neighbourhood effect on memory for order appears to be dependent on speech output processes, which provides support for psycholinguistic models of serial order.

While the neighbourhood effect replicates that found by Allen and Hulme (2006), it is surprising given that other long-term lexical variables have been shown to have no influence on memory for order. Poirier and Saint-Aubin (1996) for instance, found a significant effect of word frequency on a correct in position analysis of serial recall but found no effect on order memory, evidenced through equivalent conditional probability scores for high and low frequency sets. This finding was taken as support for a late-stage redintegration model where the order of items is selected and is then subject to redintegrative processes. While some research has found an effect of word frequency on order memory this has been disregarded and is considered an artefact of item memory. For instance, Hulme et al. (2003) found a small frequency effect on order information using a conditional probability analysis. On the basis that the frequency effect on order memory was consistent in mixed and pure lists despite the abolition of the effect of frequency on item information in mixed lists, Hulme and his colleagues concluded that word frequency does not influence order information. Further studies have supported this claim with no frequency effect on conditionalised order errors using pure lists (Allen \& Hulme, 2006; Morin, Poirier, Fortin, \& Hulme, 2006; Miller \& Roodenrys, 2009) or mixed lists (Morin et al., 2006).

If phonological neighbourhood density influences memory for order and word frequency does not, then this suggests that there is something unique about the neighbourhood variable. This suggestion is not without grounds, given that 
neighbourhood density is distinguished from other long-term memory variables in that it does not involve properties specific to a particular word like word frequency or concreteness, but is instead indicative of interaction within a corpus of lexical entries. As such, the effect on memory for order may be specific to influences that occur beyond the item level. While this result would still be indicative of a long-term memory influence prior to redintegration, it could be argued that only neighbourhood variables exert an influence at this point.

The uniqueness of effects of the neighbourhood variable in cognitive tasks from other long-term memory variables has been demonstrated previously. Other long-term memory variables such as word frequency have consistent effects on perception, production and memory (Allen \& Hulme, 2006), while neighbourhood density has opposite effects in perception and production. In perception, neighbourhood density hinders word recognition (Goldinger et al., 1989) while speech production and short-term memory tasks show facilitative effects of neighbourhood density (Allen \& Hulme, 2006).

It is possible that an effect on order memory is being masked in previous experiments due to the requirement to maintain item memory in the ISR. Given the theoretical significance of a frequency effect on memory for order, it is important to examine the effect on a more process pure test of order memory.

\subsection{Experiment 11: Investigating Other Lexical Variables: The Effects of Word Frequency and Phonological Neighbourhood Size on Memory for Order}

\subsubsection{Rationale}

The finding of a frequency effect on item memory but not on order memory has been determined using a serial recall task (Poirier \& Saint-Aubin, 1996; Miller \& Roodenrys, 2009; Morin et al., 2006; Allen \& Hulme, 2006). Thus, as previously suggested, an effect of frequency on order information could be masked by differences in item recall. Alternatively, it may be that phonological neighbourhood density is unique among long-term memory variables in producing an effect on memory for order. To examine whether word frequency influences memory for order, 
Experiment 11 used a serial reconstruction of order task with lists of large and small neighbourhood words that were either of high- or low-frequency. Manipulating both word frequency and neighbourhood density within the same experiment will also allow us to determine how these variables interact in a serial reconstruction of order task.

\subsubsection{Hypotheses}

In line with previous experiments it was expected that phonological neighbourhood density would have an effect on memory for order, with reconstruction performance better for lists of large neighbourhood words than for lists of small neighbourhood words.

If word frequency does not affect memory for order, as previously suggested, then there would be no difference in performance on the reconstruction task between lists of high frequency words and lists of low frequency words.

Predictions regarding the interaction between word frequency and neighbourhood size are neutral at this stage; however, if both neighbourhood size and word frequency facilitate memory for order then it would be expected that words that are both common and have many neighbours will be remembered better in order than the other three list types.

\subsubsection{Method}

\subsubsection{Participants}

Participants were 46 second year psychology students (17 male, 29 female) who participated as part of a subject requirement. Students included in the analysis were all native speakers of Australian English. No participant reported difficulties hearing or understanding the stimuli. 


\subsubsection{Stimuli}

Stimuli were those used in Roodenrys et al.'s (2002) Experiment 1. Stimuli were 64 single syllable CVC words that resided in one of four stimuli types. Sixteen were high frequency words $(M=145.10, S D=87.40)$ from large phonological neighbourhoods $(M=29.50, S D=8.30)$. Sixteen were words of high frequency $(M=$ $163.40, S D=137.80)$ with few phonological neighbours $(M=9.80, S D=3.10)$. Sixteen were low frequency words $(M=2.30, S D=1.60)$ from large phonological neighbourhoods $(M=28.10, S D=6.90)$. Sixteen were infrequent words $(M=2.40$, $S D=1.60)$ with few neighbours $(M=7.90, S D=2.40)$. No phonological neighbours appeared within each stimuli set but each high frequency word had one and only one low frequency neighbour in one of the other stimuli sets. Stimuli are included in Appendix B.

\subsubsection{Procedure}

New lists were created for each participant. Lists were created by arranging stimuli into lists of 6 words within each stimulus set such that no word could appear twice within a list but could appear without restriction on subsequent lists. Sixteen lists were created for each stimuli type resulting in 64 lists for each participant. These lists were then randomised to limit the effects of practice and fatigue on the results.

Participants were tested individually in a single session lasting 40 minutes. Participants performed a serial reconstruction of order task. Trials were presented via headphones connected to a Macintosh computer at a rate of one word per second. Once all 6 list items had been presented, they appeared on a 20-inch VDU, arranged vertically on the screen in a random order. Items were presented in 48-point Helvetica font as white text on a black background. Participants were asked to indicate the order of presentation by clicking on checkboxes that corresponded to list items. Participants were given strict serial reconstruction instructions and were not permitted to change a response once it had been made. Once all 6 items were selected, participants could commence the next trial via a button press. Prior to the 64 experimental trials, four practice trials were given for familiarisation with the experiment. Following 
completion of the experiment, participants were debriefed and thanked for their participation.

\subsubsection{Results}

Data were analysed using a 2 (word frequency) by 2 (phonological neighbourhood size) by 6 (serial position) repeated measures Analysis of Variance. The mean proportions correct as a function of the neighbourhood size and word frequency manipulation is represented in Figure 10.1.

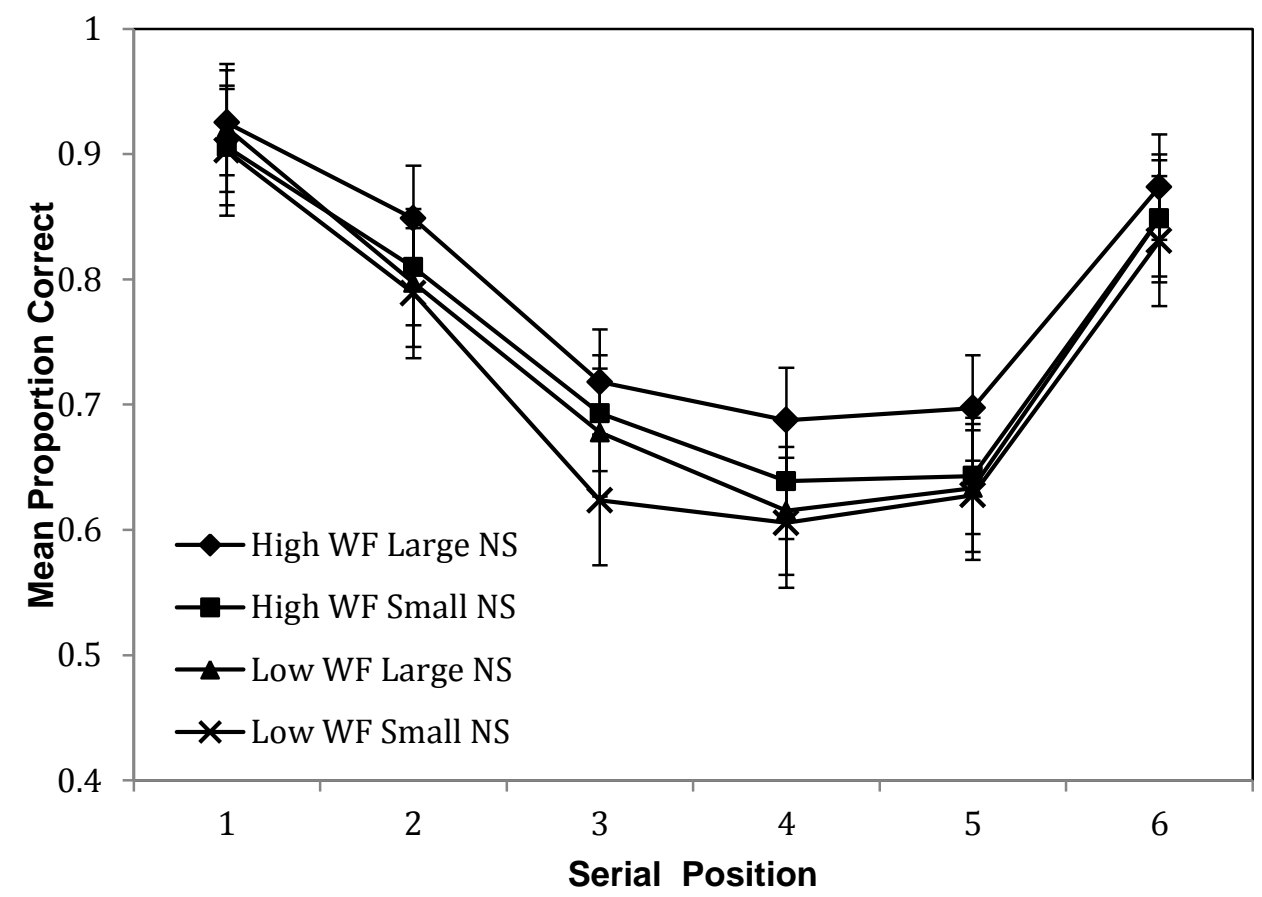

Figure 10.1. Mean proportion correct for the four stimuli conditions.

There was a significant effect of word frequency with superior performance for lists of high frequency words $(M=.77, S E=.20)$ than for lists of low frequency words $(M$ $=.74, S E=.22), F(1,45)=13.80, p<.001$.

There was a significant effect of phonological neighbourhood density, $F$ (1, $45)=8.57, p=.005$. The effect was such that reconstruction performance was better for lists of large neighbourhood words $(M=.77, S E=.21)$ than for lists of small phonological neighbourhood words $(M=.74, S E=.22)$. 
There was a significant serial position effect, $F(5,225)=68.68, p<.001$. This reflects the bowed serial position curve typically found in short-term memory tasks with superior performance for the first and last portion of the lists.

The interaction between phonological neighbourhood size and word frequency was not significant, $F(1,45)=.69, p=.411$.

The neighbourhood size by position interaction was not significant, $F(5,225)$ $=.47, p=.798$. This indicated that the size of the neighbourhood effect did not change across serial positions. There was no significant interaction between word frequency and serial position indicating that the size of the frequency effect was consistent across serial positions, $F(5,280)=1.70, p=.136$.

Finally, the three way interaction between phonological neighbourhood size, word frequency and serial position was not significant, $F(5,280)=1.31, p=.260$. This means that the effect of frequency did not change with phonological neighbourhood density across serial position.

\subsubsection{Discussion}

Experiment 11 tested the effects of both phonological neighbourhood density and word neighbourhood size on memory for order. In line with previous experiments, it was hypothesised that phonological neighbourhood size would show an effect on reconstruction performance. Further, following previous findings (i.e., Poirier \& Saint-Aubin, 1996; Allen \& Hulme, 2006) it was expected that word frequency would not affect memory for order. Results showed support for the first hypothesis, with superior memory for order for lists of large phonological neighbourhood words compared with small phonological neighbourhood lists. However, contrary to the second hypothesis there was a significant effect of word frequency on memory for order, with memory for order better for lists of high frequency words than for lists of low frequency words. This finding of a frequency effect indicates that the long-term memory influence on memory for order is not unique to phonological neighbourhood density, and further suggests that long-term memory may exert an influence earlier than suggested by late-stage redintegration models. The reason for the difference between the current results and previous results (i.e., Poirier \& Saint-Aubin, 1996; Morin et al., 2006; Allen \& Hulme, 2006) may be 
due to task differences between ISR and serial reconstruction, namely that ISR necessarily confounds item and order memory. Another possible explanation for the difference between Poirier and Saint-Aubin (1996) and the current results is that Poirier and Saint-Aubin used mostly two syllable words while the current experiment used monosyllabic words. This may have increased the reliance on item information in Poirier and Saint-Aubin's experiment as more information per word was required to correctly recall the item in the correct position.

However, it is clear from other research that task differences may not be the only reason for the discrepancy. Hulme et al. (2003) for instance, reported a small but significant effect of word frequency on memory for order using a conditional probability analysis of an ISR task. This suggests that word frequency does influence memory for order, and this can be detected in a serial recall task, although is likely to be attenuated due to the item confound. Given that conflicting findings have been identified within the same task, an explanation for the conflict must be sought. While Hulme et al. (2003) mostly used bisyllabic words, they reduced the requirement to remember item information by using a closed and small stimuli set of only 16 items. This makes the task more one of order memory.

Yet another possibility is that Poirier and Saint-Aubin's (1996) study and Morin et al.'s (2006) study were conducted with French words, whereas the current study and Hulme et al.'s (2003) study were conducted with English words. There may be important differences in the characteristics of French and English words that contribute to the conflicting findings. While many differences do appear, for instance English is a stress-timed language while French is a syllable-timed language (Baus et al., 2008); it is not immediately clear how this or other differences may cause the effect to occur in English but not in French.

Evidence does suggest that linguistic processes may operate differently depending on the language. For instance, while the Spanish language enjoys similar relationships between frequency, word length and phonological neighbourhood size as the English language, these variables influence cognitive processes differently to the influences occurring in English. Vitevitch and Rodriguez (2005) were interested to see whether the differences in the languages would lead to differences in the effects on linguistic processing. Using an auditory lexical decision task with native Spanish speakers, they found that responses for words from dense phonological 
neighbourhoods were faster and more accurate than with sparse phonological neighbourhoods, which is the opposite of the effect found in English. Further, Vitevitch and Stamer (2006) found faster naming times for words from sparse phonological neighbourhoods than dense phonological neighbourhoods in Spanishspeaking participants. This is opposite to the effect found in English and is consistent with the finding from Vitevitch and Rodriguez (2005).

Further, Thorn et al. (2002) found evidence for some differences in the influence of long-term memory variables in French and English. They examined French/English and English/French bilinguals on ISR and serial recognition of order while manipulating lexicality and phonological similarity. While the word familiarity advantage, interpreted as a frequency effect, was similar across French and English native speakers, the lexicality effect was greater for English native speakers than for French native speakers. Despite differences identified between English and French native speakers in serial recall, these differences were not identified in serial recognition. This suggests that linguistic differences may result in different effects in short-term memory for items but typically do not manifest as effects on memory for order. This is inconsistent with findings from Experiment 11, but may be due to the lack of sensitivity of the recognition task. It would be conducive to the interpretation of the results of Experiment 11, to examine differences in reconstruction performance on native English and native French speakers.

An alternate explanation of the disparity between Poirier and Saint-Aubin's (1996) result and the current finding was that the former did not control for phonological neighbourhood density. Their stimuli were 216 words that were either high, medium or low frequency words. The words were arranged into lists of phonologically similar words, with phonological similarity occurring on the rime component of the words. The same words were then also arranged into lists of 6 words such that no phonologically similar words could appear within the same list. There was no indication of neighbourhood size of the lists, although it is evident that each word had at least 6 phonological neighbours, and that these phonological neighbours appeared within the stimuli set. To address the inconsistency between the current results and that of Poirier and Saint-Aubin (1996) further testing needs to be conducted using the same task conditions as the current experiment but with French 
words. Hulme et al. (2003) also did not control for neighbourhood density; however all but two of their stimuli (statement and treatment) were phonologically distinct.

Regardless of the reason for the discrepancy, the current results suggest that word frequency, along with phonological neighbourhood size, has an influence on memory for order, and not just on item memory. This indicates that despite differences in the mechanisms behind frequency effects and neighbourhood effects, both influence memory for order, pointing to a long-term memory influence occurring prior to the reconstruction of items. This is difficult to reconcile with models of memory for order that place the interface with long-term memory at the point of recall, after items have been selected in order. The current results indicate that a longterm memory influence occurs earlier than originally modelled but do not elucidate the exact location of this interaction.

Thorn, Frankish, and Gathercole (2009) suggest that the long-term memory effects could occur at multiple points in the short-term memory process. They examined long-term memory effects such as lexicality, word frequency, and phonotactic frequency in short-term memory using ISR and serial reconstruction. They suggested that if effects were significantly attenuated or removed in reconstruction then this would pinpoint the locus of their effect to redintegrative processes occurring during recall. However, if the effects persisted then this would suggest that long-term memory is able to influence short-term memory during either the encoding or storage of the traces, as predicted by pre-retrieval accounts (i.e. Martin, 2007). Thorn and her colleagues found that some, but not all, of the effects were significantly reduced in reconstruction compared with recall. Specifically, word frequency and lexicality were greatly reduced in the reconstruction task while phonotactic frequency persisted. Further, they demonstrated that the phonotactic frequency effect in reconstruction could not be attributed to differences in the rates at which high and low phonotactic non-words could be articulated and was not due to differences in the speed with which the items were read. Instead, the researchers suggested that the results indicate multiple mechanisms that operate at different points in the memory process. One process operates to strengthen the viability of the trace within short-term memory. This occurs during encoding and maintenance and is primarily responsible for the persistence of the phonotactic frequency effect in serial reconstruction. The second process serves to redintegrate degraded traces during 
recall. Based on their results, Thorn and her colleagues suggested that word frequency and lexicality are primarily influenced by the latter process. However, the results of the current thesis provide evidence that word frequency and phonological neighbourhood density also exert an influence during the pre-retrieval stage. Thorn et al. (2009) conceded that word frequency may influence the initial state of the memory trace but may not have shown an influence in reconstruction due to issues with task sensitivity. It is perhaps unsurprising that phonological neighbourhood density follows the same patterns as phonotactic frequency on order memory given the similarities between the variables (Vitevitch et al., 1999). The current experiments confirm that frequency does influence reconstruction and suggests that this variable influences the stability of the trace.

It is unclear from Thorn et al. (2009) how these long-term memory variables influence the stability of traces. It is possible that the effect occurs during encoding as evidence suggests that long-term memory is activated during this stage. For instance, large neighbourhood words are more difficult to perceive in noise, and take longer to recognise during auditory presentation due to competition at the lexical level (Goldinger et al., 1989). It may be that the increased time it takes to perceive words from large neighbourhood words affords them stronger overall representation in the memory set. As the neighbouring lexical items are partially activated during presentation (in order to provide some lexical competition), it is possible that these items maintain some lower level of activation and are able to provide an additional cue at recall. Alternatively, it is possible that the effect occurs during maintenance, which involves the cyclical reactivation of the list items. As item memory is superior for large neighbourhood and high frequency words, recalling these items during rehearsal demands fewer resources than rehearsing small neighbourhood and low frequency words. This may leave more resources available for remembering the order information associated with these items. These explanations will be explored further and with respect to models of memory for order in the discussion chapter of this thesis. 


\section{Chapter Eleven}

\section{A Recapitulation of Current Research}

\subsection{An Overview of the Research Project}

Previous research on the effect of lexico-semantic variables on short-term memory led to the conclusion that such effects only exert an influence on item memory. Such findings spurred the theoretical advancement of short-term memory models, most notably with the development of a two-stage redintegration process. In these models, order memory is maintained over the short-term without interference from long-term memory. At recall, items are output in order and undergo a deblurring process to restore degraded traces. It is during this recall stage that current models of memory pinpoint the long-term memory influence on short-term recall. Accordingly, lexico-semantic variables should not influence memory for order.

Roodenrys et al. (2002) challenged this finding by showing an influence of phonological neighbourhood size on short-term memory for order. Specifically, large phonological neighbourhood words were more likely to be recalled in the wrong position in mixed lists. A queue-jumping hypothesis was proposed where long-term memory was able to contribute to the activation that determined the order an item was selected from short-term memory. Further contention was introduced with Allen and Hulme's (2006) finding of a neighbourhood effect on memory for the order of pure lists. Specifically, they found that words from large neighbourhoods were remembered better in their serial order than words from small neighbourhoods. This is incompatible with the queue-jumping hypothesis, which would suggest that, while amongst similarly activated items, order recall would not be affected by neighbourhood density. While contrary to the proposed mechanism of the influence, namely the queue-jumping hypothesis, the finding of an effect in pure lists remains indicative of a long-term memory influence on ordered recall.

The aim of this thesis was to investigate the neighbourhood effect on memory for order using tasks that varied in their requirement to maintain item and order information. The finding of a phonological neighbourhood effect on order memory 
would have important theoretical implications for models of memory for order. The following is a summary of the findings of the studies in this thesis.

\subsection{The Phonological Neighbourhood Effect on Memory for Items}

\subsubsection{Mixed Lists}

The phonological neighbourhood effect on memory for items was examined in Experiment 1 using an ISR task with mixed lists. On the basis of the results of Roodenrys et al. (2002), it was expected that large neighbourhood words would be more likely to be recalled in a different position than small neighbourhood words. In line with the queue-jumping hypothesis, large neighbourhood words were expected to be recalled ahead of their serial position, effectively displacing small neighbourhood words. In contrast to this finding, Experiment 1 failed to reveal an effect of neighbourhood density on overall item recall in mixed lists. This may reflect something specific about the stimuli selected, given that the neighbourhood effect has been found previously in mixed lists in ISR (Roodenrys et al., 2002). Alternatively this may reflect important differences between the Roodenrys et al. (2002) experiment and the current experiments. Experiment 1 used lists of alternating large and small neighbourhood words, while Roodenrys et al. (2002) did not exert such stringent control on how items were arranged within the lists. The inability to find an effect in mixed lists in Experiment 1 may reflect some reciprocal influence of large and small neighbourhood words on one another. That is, small neighbourhood words within the lists may hinder memory of large neighbourhood words, while large neighbourhood words may assist memory of small neighbourhood words; a pattern that has been observed with word frequency manipulations (Hulme et al., 2003). This may not have been identified in Roodenrys and his colleagues experiment because they did not employ the same list composition paradigm.

To evaluate these possibilities, Experiment 3 examined the effect of phonological neighbourhood density on ISR of pure lists. The findings of Experiment 3 will be discussed before Experiment 2 in this section as the second experiment does not examine item memory. Given the robustness of the effect in other experiments, if pure lists also did not elicit a neighbourhood effect then it was possible that the 
inability to find an effect in mixed lists is a function of the stimuli used. If a neighbourhood effect was found in pure lists, it would be of value to examine the relative levels of performance of mixed and pure lists to ascertain whether the null effect in mixed lists was a result of some reciprocal influence of the list components.

\subsubsection{Pure Lists}

The phonological neighbourhood effect on ISR was examined in Experiment 3 using pure lists of words from large and small neighbourhoods. As expected, there was a significant effect of phonological neighbourhood density when scoring items according to both correct-in-position and free recall criteria. Aggregate data revealed that this was in the expected direction with greater recall of items from large phonological neighbourhoods than from small phonological neighbourhoods. The finding is consistent with Roodenrys et al. (2002) and Allen and Hulme (2006) and can be interpreted as a long-term memory influence on short-term memory. Importantly, the finding from Experiment 3 indicates that the null effect from Experiment 1 was not due to problems with the stimuli.

Whether the finding from mixed lists reflects large neighbourhood words supporting recall of the small neighbourhood words, or small neighbourhood words hindering recall of large neighbourhood words can be ascertained by examining relative performance levels of pure lists (Experiment 3) and mixed lists (Experiment 1). Looking at the probability of recall across serial positions for both types of list composition, it is evident that recall performance in the mixed lists is most similar to performance for large neighbourhood words in the pure list condition. This suggests that the superior memory for large neighbourhood words assists the recall of small neighbourhood words, perhaps by reducing the overall cognitive load associated with recalling lists composed entirely of small neighbourhood words. While this refutes item-based accounts, which would expect a saw-toothed serial position curve, it does not entirely fit with list-based accounts. List-based accounts would predict that the effect would disappear in alternating lists, but would also anticipate that performance would fall at a level intermediate to that of pure lists. Instead, this study showed performance on mixed lists is equivalent to pure lists of large neighbourhood words, a pattern that is similar to that found for lists of alternating word length. Hulme, Surprenant, Bireta, Stuart, \& Neath (2004) found that the significant advantage for 
short words over long words found in pure lists was abolished in mixed lists Further, Hulme and his colleagues found that mixed lists of long and short words were recalled at a level similar to that of pure lists of short words. This is similar to the pattern observed here with neighbourhood density. Hulme et al. (2004) argued that their result reflected an effect of phonological complexity with long words being more complex and therefore more difficult to remember than short words. They proposed that mixed list sets of long and short words were more distinctive than pure lists. It is unclear how this can be explained within the context of neighbourhood density as the difference between large and small neighbourhood words is not immediately obvious to the participant and therefore cannot be used as a conscious mnemonic cue, unlike word length which is highly salient. For this explanation to suit the pattern of results found with neighbourhood density, distinctiveness must assist recall on a subconscious level, perhaps due to differences in the amount of supportive activation given to large and small neighbourhood words during the memory process.

To summarise, these experiments confirm an effect of phonological neighbourhood density on short-term item memory. This effect is evident in pure lists but disappears in mixed lists. The finding of an effect on item memory confirms that previously found in the literature (Roodenrys et al., 2002; Allen \& Hulme, 2006). Experiments investigating the primary aim of the thesis, examining the neighbourhood effect on memory for order, will now be reviewed.

\subsection{The Phonological Neighbourhood Effect on Memory for Order}

\subsubsection{Mixed Lists}

In accordance with findings from Roodenrys et al. (2002) and the queuejumping hypothesis, it was expected that the phonological neighbourhood effect on memory for order would manifest in mixed lists as opposed to pure lists. The phonological neighbourhood effect on memory for order of mixed lists was investigated using three methods; a conditional probability analysis of serial recall data (Experiment 1), a serial order recognition task (Experiment 2) and a serial reconstruction of order task (Experiment 4). There was no significant effect of neighbourhood density in any task, contradicting the queue-jumping hypothesis. The 
finding suggests that long-term memory does not contribute to order memory, at least not in the manner proposed by Roodenrys et al. (2002). Again, the finding of an effect on mixed lists obtained by Roodenrys and his colleagues may be due to the specific paradigm used by the researchers and may reflect that the effect is dependent on list composition.

\subsubsection{Pure Lists}

Predictions for experiments using pure lists of large and small phonological neighbourhood words were less clear. The queue-jumping hypothesis would predict that there would be no order effect on pure lists of large and small neighbourhood words. However, Allen and Hulme (2006) found a significant effect on the number of order errors reported using pure lists in an ISR task. They found that pure lists of large neighbourhood words were more likely to retain their serial position than words from small phonological neighbourhoods, while controlling for the total number of items recalled post hoc.

Given these contradictory findings, the investigations relating to pure lists were exploratory. The effect of neighbourhood density on memory for order in pure lists was examined in Experiment 3 by looking at the proportion of order errors in a serial recall task and performance on a serial recognition task. No significant effect of neighbourhood density was found using either measure, suggesting that perhaps neighbourhood size does not influence memory for order. However, it was possible that the lack of significant effect in ISR was still due to confounds with item recall. Further, it is possible that the recognition task was not sensitive enough to detect order errors naturally occurring in memory, as the task only involved identification of the transposition of two items per list. To address this, Experiment 5 used a more "process pure" test of order memory, the serial reconstruction of order task, to examine the effect of neighbourhood density. A significant effect was found, with superior order memory for lists of large neighbourhood words compared with lists of small neighbourhood words. This effect was replicated in four experiments, signifying a robust effect. Experiment 7 indicated that the neighbourhood effect could not be explained by differences in reaction time at output and that the effect could not be explained due to differences in word length between large and small neighbourhood sets. 
The finding of a neighbourhood effect in serial reconstruction of pure lists refutes the queue-jumping hypothesis, at least in the context of alternating lists, but still suggests that long-term memory is contributing to the short-term memory of order in some way. It is possible from the results of these experiments that phonological neighbourhood size is able to contribute to representations in a manner that makes them more stable. Stability of representations may be a result of better encoding of order information, or may be due to processes occurring during the maintenance of items.

DeLosh and McDaniel (1996) suggest that order encoding is affected by the amount of cognitive resources required to process item information. That is, for items that are easily encoded, such as common words, coding of order information will occur with a certain degree of efficiency. For items that are difficult to process, such as uncommon words, additional resources will be required to ensure the item information is processed and this detracts from resources ordinarily allocated to order processing. In processing words from large and small phonological neighbourhoods two different effects have occurred depending on whether the items are presented in visual or verbal form (Yates, 2005). Large phonological neighbourhood words are afforded an advantage when processing is visual, but coming from a large neighbourhood encumbers speech perception. According to DeLosh and McDaniel's (1996) order-encoding framework, differential order effects should appear depending on how items are presented to participants. Specifically, if items are presented visually, there should be better order memory for large neighbourhood words as these are processed more efficiently than small neighbourhood words. If words are presented orally, there should be an advantage for small neighbourhood words as these are processed more rapidly than large neighbourhood words leaving additional resources for order encoding. Evidence from the current experiments does not support this hypothesis. Experiment 7 showed a clear advantage for large neighbourhood words on order memory when items were presented orally, while Experiment 5 showed an advantage for large neighbourhood words on memory for order when items were presented visually. While the failure to find support for the order-encoding framework does not rule out the possibility that the influences identified in this thesis are due to encoding of order, alternative frameworks need to be constructed to show how order encoding accounts for the neighbourhood finding. 
An alternative explanation to a differential effect at encoding is that the greater stability of the items occurs due to processes operating during the maintenance of items in memory. To examine this, it is of value to return to the memory span equation identified by Hulme et al. (1991), which was discussed in Chapter 3 and given again here;

$$
s_{i}=c+r_{i} \tau \quad \text { [equation 2.1] }
$$

Where $s_{i}$ is span, $c$ corresponds to the contribution from long-term memory, $\tau$ is an estimate of decay, measured in seconds, and $r_{i}$ is the speech rate for item $i$.

The speech rate and decay parameters govern the maintenance of items in short-term memory, while $c$ represents the contribution from long-term memory that occurs once the order of items has been retrieved. Given that order information is retrieved before the interaction with long-term memory $(c)$ according to the reconstruction hypothesis, the influence of neighbourhood density on memory for order must occur on either the decay parameter $(\tau)$ or the speech rate parameter $\left(r_{i}\right)$. While this original equation has these two processes as independent of long-term memory, the results of this thesis suggest that long-term memory must influence one or both of these parameters.

Saint-Aubin and Poirier (1999a) attribute order errors to trace interpretation problems at recall. That is, if an item's unique phonological features have decayed to the extent that it is no longer distinct from other list items then it may be erroneously selected for retrieval and reconstruction. However, it is unclear how, without contribution from long-term memory, large and small neighbourhood words would decay at different rates. Further, while pronunciation rate has not been directly tested in this series of experiments, Roodenrys et al. (2002) found a significant effect of phonological neighbourhood density on speech rate. However, by including the variable as a covariate, they ruled out the possibility that the significant effect of neighbourhood density on item memory in their experiment was due to speech rate differences between stimuli. It is important to note that Roodenrys and his colleagues ruled out articulation rate as an explanation of the neighbourhood effect on item information, and that this remains as a possible explanation of neighbourhood effects on order information. Whether the effect of phonological neighbourhood density on 
memory for order is due to some kind of speech production process (i.e. the $r_{i}$ variable) was assessed further in this thesis by removing the influence of speech production using articulatory suppression.

\subsubsection{The Effect of Articulatory Suppression}

Articulatory suppression was used to determine whether the phonological neighbourhood effect identified in the short-term memory for order tasks could be attributed to speech production processes. Articulatory suppression is thought to remove the opportunity to covertly articulate items by requiring overt articulation of an irrelevant word. As covert and overt speech occur at a similar rate (Landauer, 1962), it is unlikely that participants would have the speech production resources to also covertly articulate list items. The impact of articulatory suppression was examined in two experiments; Experiment 8 and Experiment 9. In both experiments, suppression was required throughout presentation, and continued through recall. No effect of phonological neighbourhood size was found in either experiment. Given that neighbourhood density does not influence span when articulatory suppression is required suggests an independent long-term memory influence on short-term memory for order that occurs during rehearsal, involving the sub-vocal articulation of the items.

This conclusion that long-term memory exerts an influence on the rehearsal of items in short-term memory via the speech production system was based not only on the current results but also on previous literature. First, evidence suggests that rehearsal is governed by systems responsible for speech production. Paulesu, Frith, and Frackowiak (1993) attempted to identify the neural correlates of verbal short-term memory by presenting English and Korean letters visually to participants while monitoring neural activity using a Positron Emission Tomography (PET) scan. They noted that according to the working memory model the English letters would automatically be transcribed to a phonological code using the articulatory loop, while the Korean letters could only be stored as visual images given that participants were unable to speak Korean. Thus the Korean letters were used to provide a baseline. In the first task, participants were required to learn a string of letters, to engage both the articulatory loop and the phonological store. In the second experiment, participants were asked to make rhyming judgements on pairs of visually presented letters; a task 
purported to involve the articulatory loop but not the phonological store. Separate cortical areas were activated in both tasks, lending credence to the idea of separable phonological store and loop systems. The tasks were associated with activation in Broca's area, an area usually associated with speech and language production. Paulesu et al.'s (1993) results are indicative of a neurologically separate phonological store and articulatory loop and are indicative of Broca's area as a neural underpinning for subvocal rehearsal. Smith and Jonides (1999) extended the role of Broca's area to overt rehearsal as well as covert rehearsal. Further, Caplan and Waters (1995) found evidence that patients who had lost the ability to construct a speech-motor pattern had no capacity to rehearse, while Baddeley and Wilson (1985) report evidence from patients who have lost control of their speech musculature but may still be able to covertly rehearse. This is compatible with the view that rehearsal involves the cyclical reactivation of the speech output plan (Woodward et al., 2008).

Second, there is a link in the literature between rehearsal and long-term memory, with evidence suggesting that active rehearsal is necessary for sequential learning. Ellis and Sinclair (1996) for instance, note that short-term rehearsal of sequential information allows for long-term consolidation of verbal memory. Kidd and Greenwald (1988) showed that attention and rehearsal were necessary for the reproduction of a repeated sequence of items. Further, other researchers have identified a link between memory for serial order and long-term memory. Majerus, Poncelet, Greffe, and Van der Linden (2006) identified that memory for order was related to new word learning in 4- and 6- year olds. Gupta (2003) found that this relationship between vocabulary learning and the serial order component of immediate serial recall persisted in adults. Gupta (2003) suggested a link between sequential memory and the mental lexicon, where sequential memory represents a temporary pattern of activation across items in long-term memory. This model and its implications will be discussed in more detail in Chapter 12. While this suggests at least a unidirectional link between memory for order and long-term memory, the current results suggest a reciprocal link between these systems. That is, sequence memory is necessary for encoding items into long-term memory, and long-term memory is able to assist with the maintenance of items in a sequence in short-term memory.

There is some neuropsychological evidence for the activation of long-term memory during rehearsal. Collette et al. (2001) examined the neural underpinnings for 
words and non-words using PET technology. The researchers presented French words and French phonotactically legal non-words. Collette et al. (2001) found activation in Broca's area during the maintenance of both words and non-words, consistent with Paulesu et al.'s (1993) finding. The rehearsal of words was associated with an increase in activity in cerebral areas involved in the maintenance of semantic and lexical long-term knowledge. These areas are the right superior temporal gyrus (BA 22) and the right middle frontal gyrus (BA 9).

The results are neutral with regard to the extent of the relationship between verbal memory and language process. That is, it is unclear whether memory and language use common processes, as purported by the psycholinguistic view, or whether language processes mediate interactions between specified memory systems. However, it is clear from current results that speech production processes do play some role in the interaction between permanent item representations and the temporary maintenance of a sequence of items.

\subsection{The Word Frequency Effect on Short-Term Memory for Order}

The word frequency effect was introduced to ascertain whether the long-term memory influence on memory for order is specific to the neighbourhood variable, or is a more general effect of lexico-semantic variables that had not been previously identified. The effect of word frequency on memory for order was examined in Experiment 11 using serial reconstruction. As well as showing a significant neighbourhood effect, Experiment 11 revealed a significant effect of word frequency. Further, this effect was independent of phonological neighbourhood size.

The effect of word frequency on memory for order has contradictory findings in the literature. Hulme et al. (2003) for instance, showed a small but significant effect of word frequency on memory for order. However, Poirier and Saint-Aubin (1996) and Allen and Hulme (2006) found no effect of word frequency on conditionalised order errors in a serial recall task. The Poirier and Saint-Aubin (1996) study was conducted with French words, which as previously discussed, may differ in important ways from English words. Indeed other studies of effects in other languages have identified that different languages may produce different effects (Vitevitch \& Rodriguez, 2005; Vitevitch \& Stamer, 2006). 
The disparity between the current results and those observed by Allen and Hulme (2006) cannot be explained in terms of language differences. These previous studies may have failed to find an effect of word frequency on memory for order as the ISR task used in these experiments was not sensitive enough. Support for this comes from Nairne and Kelley (2004), who examined the effect of long-term memory variables on item memory and order memory separately using process dissociation. They found a small but significant effect of word frequency on memory for order using this method. They note that because this effect is small it may not be observable in tasks where the requirement to maintain item information is paramount. One factor preventing direct comparison between these experiments is that Nairne and Kelley (2004) used a delayed recall procedure with a filled retention period. This makes the task one of long-term memory, and results are not directly analogous to those in shortterm memory. It would be of value to replicate the process dissociation procedure within the realm of short-term memory to determine whether the failure to find a frequency effect in all tasks is due to the size of the effect and the degree of task sensitivity.

While there are caveats with Nairne and Kelley's (2004) conclusion that task sensitivity may mask order effects in some tasks, other researchers have made comparable deductions in short-term memory. Thorn et al. (2009) came to a similar conclusion regarding their failure to find an effect of word frequency, while revealing a significant effect of phonotactic frequency, on memory for order. They suggested that multiple mechanisms may be responsible for the impact of long-term knowledge on ISR. One that occurs during redintegration and another that provides an interaction between short- and long-term memory representations during encoding and/or storage of items. They conclude that some long-term memory variables, such as phonotactic frequency influence both processes. They further conclude that it is likely that word frequency also influences both processes but its effect on the pre-retrieval process may be masked due to task sensitivity issues. The results of the current experiment support this, and demonstrate that both word frequency and phonological neighbourhood density influence the memory process prior to the redintegration of items. 


\subsection{Conclusion}

The findings from the current series of experiments indicate that phonological neighbourhood size has an effect not only on maintaining item information in shortterm memory, but also in maintaining the order of the items. The additional finding that word frequency also influences memory for order suggests that this is due to a general influence of long-term memory on short-term memory for order. It is possible that previous attempts to locate lexical effects on memory for order may have been unsuccessful due to the nature of the tasks used. 


\section{Chapter Twelve}

\section{Implications of the Current Findings and Future Research Directions}

\subsection{Introduction}

The consolidation of the results of the experiments reported in this thesis suggests that phonological neighbourhood density and word frequency both exert an influence on memory for order, which is indicative of a more general effect of longterm memory on short-term memory for order. The proposed mechanism behind this effect is an interface with long-term memory that occurs prior to the redintegration of items. This poses a challenge for current models of memory for order. This chapter recapitulates predictions made by these models and an effort is made to reconcile findings with these approaches to memory for order. Suggestions for future research will also be discussed.

\subsection{Implications for the Retrieval-Based Hypothesis}

The retrieval-based hypothesis pinpoints the locus of order errors to trace interpretation issues during redintegration (Saint-Aubin \& Poirier, 1999a). The theory predicts order errors would occur if items within a list share many of the same phonological features or if enough unique phonological information has been lost due to decay or interference. While item memory for small phonological neighbourhood words is inferior to that of large phonological neighbourhood words, this has been attributed to processes occurring at recall rather than any quantifiable differences in the decay parameter between large and small neighbourhood words. To reconcile the retrieval-based hypothesis in its current state with this pattern of results is to suggest that word frequency and phonological neighbourhood size influence the degradation of a trace either instead of, or in addition to, influencing the reconstruction of a trace. This necessitates a link with long-term memory that occurs during the maintenance of 
items in short-term memory, or alternatively could suggest that order information is more strongly encoded for large neighbourhood words than it is for small neighbourhood words. As a result large neighbourhood words may be more resilient to the effects of decay. With regards to the order-encoding framework outlined in Chapter 11 (DeLosh \& McDaniel, 1996), it is unclear how these effects could be explained in terms of encoding during list presentation as large neighbourhood words typically take longer to perceive than small neighbourhood words (Goldinger et al., 1989). However, it is possible that redintegration occurs during rehearsal as well as during recall, a concept that has been suggested by other researchers (e.g., Turner, Henry, Smith, \& Brown, 2004). If item information belonging to large neighbourhood words is more easily retrieved and/or redintegrated then more resources will be available for re-coding order information for these items. This is similar to the orderencoding hypothesis but pin-points the locus of the neighbourhood effect to order encoding advantages during rehearsal rather than during the initial encoding of the items.

An alternative explanation is that mechanisms other than redintegration are responsible for long-term memory effects on short-term memory for order. Thorn et al. (2009) found that while redintegration was still a valid explanation of most longterm memory effects on short-term memory, it was unable to capture all effects identified in the literature. They proposed that in addition to redintegration there exists a pre-retrieval link that operates in the manner described by psycholinguistic models of memory (i.e., Martin, 2007; Gupta, 2003).

Regardless of the mechanism or mechanisms responsible for the effect, it is evident from the current results that the traditional retrieval-based hypothesis is in need of revision. This has important implications for models of memory for order as many employ some instantiation of the retrieval-based hypothesis. The aim of the following section is to review these models and attempt to reconcile them with the current results. 


\subsection{Implications for Models of Memory for Order}

\section{Chaining Models}

Chaining models store order information via associations between neighbouring items, where each item cues the recall of the next item. The most prominent theory that adopts a chaining approach to memory for order is Murdock's (1982) Theory of Distributed Memory (TODAM). As outlined in Chapter 6, items are added to a memory vector as they are presented, and order information is modelled by convolving neighbouring item vectors with one another and then adding this convolution to the item vector. At recall, each item recalled cues an approximation of the successive item. This approximation then undergoes a deblurring process. Order errors occur when the approximation of one item is a closer representation of a different list item. According to TODAM2 (Lewandowsky \& Murdock, 1989), longterm memory influences the strength of encoding. As such, order memory may be superior for large neighbourhood words because these are encoded more strongly in the memory vector. When "unpacking" this information at retrieval, the approximation of each item will likely be a strong representation of the correct item because of the advantage at encoding. This would not only increase the likelihood that the item information will be deblurred correctly, but would also reduce the likelihood that the item would transpose. This may also be able to explain the removal of the neighbourhood effect in mixed list conditions. Given a list $L_{1} S_{2} L_{3} S_{4} L_{5} S_{6}$, where $S$ is a small neighbourhood item and $L$ is a large neighbourhood item, if the approximation of $L_{l}\left(L_{1}\right.$ ') was a strong approximation of the item $L_{l}$ then it would be likely that the correct word would be recalled in the correct position. The recall of the correct word would provide a strong cue for the retrieval and subsequent deblurring of item $S_{2}$, and so on. In this way, the stronger activation of large neighbourhood words could help to constrain the movement of small neighbourhood words. Further, the poorer encoding of small neighbourhood words could potentially harm memory for large neighbourhood words by not providing a strong cue. If large neighbourhood words were able to provide a stronger influence on small neighbourhood words, then it would be expected that performance on mixed lists would be closer to the performance of pure lists of large neighbourhood words. This was found when 
comparing performance levels between mixed lists and pure lists between-subjects (Experiment 4 and Experiment 5). However, when comparing performance levels between mixed and pure lists within-subjects revealed performance for mixed lists equivalent to that of small neighbourhood words in pure lists, a finding difficult to reconcile with the TODAM model. It was suggested that the difference between the amalgamation of Experiment 4 and Experiment 5, and the results of Experiment 6, could be due to cohort differences in the between subjects analysis. Thus it is likely that the relative performance levels observed in Experiment 6 accurately represent the true nature of the effect, although replication of this finding is needed for validation. For this to be reconciled with TODAM, which would predict intermediate performance for mixed lists, it would need to be assumed that the magnitude of the detrimental influence of small neighbourhood words is greater than the facilitative influence of large neighbourhood words. It is possible to see how this can occur in alternating list of large and small neighbourhood words given that an early error in the list due to a weak cue provided by a small neighbourhood word could disrupt the recall of the entire list, especially in tasks such as the ones used here where repeats in recall are not tolerated. For instance, if, in the list $L_{1} S_{2} L_{3} S_{4} L_{5} S_{6}$, item $S_{2}$ incorrectly cues item $S_{4}$, causing this item to be recalled in position 3, then erroneous recall will likely continue throughout the list even if $S_{4}$ correctly cues the next item $L_{5}$ at least in terms of absolute serial positioning. A closer examination of the level of relative and absolute recall could go some way to addressing this possibility.

While TODAM makes no specific assumptions with regards to the role of articulatory suppression, it is possible that articulatory suppression disrupts the resources needed to adequately encode items, and in terms relating to TODAM convolve items into the memory vector. This would result in an overall decrement in performance, as observed, although it is unclear why articulatory suppression would differentially influence large and small neighbourhood words, resulting in the removal of the phonological neighbourhood effect. Indeed, Gregg, Freedman, and Smith (1989) showed that frequent and infrequent words are affected by articulatory suppression in the same way, with a frequency advantage persisting under suppression conditions despite an overall decrement in recall (see also, Miller \& Roodenrys, 2012). 


\section{Positional Models}

In positional models, items are coded according to their absolute serial position, stored via associative links between the item and its position. These models could explain the neighbourhood effect on memory for order if long-term memory were able to influence the quality of the item to position association. For instance, OSCAR employs a context-timing signal to maintain order but states that order errors occur at the point of retrieval. According to this model, the likelihood of an error depends on the quality of the retrieval cue and whether the cue adequately represents the item. For the phonological neighbourhood effect to influence order, neighbourhood size would need to influence either the quality of the retrieval cue, or the specificity of the cue's representation of a particular list item (Brown et al., 2000).

If large neighbourhood words were able to influence either variable, then, at least for serial recall, it would be expected that large neighbourhood words will be recalled better than small neighbourhood words even when they were presented within the same lists. That is, it would be expected that a saw-tooth serial position curve be obtained when large and small neighbourhood words were presented in an alternating fashion in the same lists. This would be indicative of an item level effect rather than a list level effect. In serial reconstruction, this scalloped serial position curve may not be expected if it is assumed that superior positional memory for large neighbourhood words could assist the successful reconstruction of small neighbourhood words. That is, the more stable large neighbourhood words would constrain the movement of the less stable small neighbourhood words in mixed lists. This would lead to the prediction of performance levels of mixed lists being either at, or near, the performance level of pure lists of large neighbourhood words. Again, a comparison of performance level between mixed lists and pure lists (Experiment 6) support this, while the amalgamation of Experiment 4 and Experiment 5 refute this, with performance on mixed lists being greater to that of pure lists of large neighbourhood words. While further experiments need to be conducted to quantify these outcomes given the differences between the within- and between-subject results, they seem to be most consistent with non-positional accounts. 


\section{Ordinal Models}

In ordinal models, order is stored via the relative activation levels of list items, with a decreasing gradient of activation offered to successive items. According to one popular ordinal model, the primacy model (Page \& Norris, 1998), order errors occur due to noise in the gradient of activation. In the primacy model, noise is modelled by adding zero-mean Gaussian noise to the activation of each item prior to retrieval (Page \& Norris, 1998). It is assumed that this noise is random, and is not influenced by extraneous factors such as long-term memory. If a non-random noise parameter were modelled, it may be possible to explain long-term memory effects on short-term memory for order. If less noise was added to the selection mechanism for large neighbourhood words, perhaps due to their greater specificity in long-term memory, then it would be expected that order memory would be better for large neighbourhood words. An alternative is that long-term memory could influence the distinctions between the relative activation levels of neighbouring words. If lists of large neighbourhood words were presented on a steeper gradient of activation then it may be expected that they would be less affected by the noisy selection process and thus recalled better in order. Given that activation must surpass a baseline for a word to be recalled, this alternative necessarily assumes that the activation level proffered to large neighbourhood words must be higher than that available to small neighbourhood words.

This has certain implications for predictions regarding mixed lists. If a stronger activation was given to large neighbourhood words than small, then it would be anticipated that words from large phonological neighbourhoods would be recalled ahead of their serial position if by doing so, they would displace words from small phonological neighbourhoods. This is reminiscent of the results from Roodenrys et al. (2002) but is not compatible with the findings from Experiments 1 and 2, or from the results using mixed lists in serial reconstruction of order task (Experiment 4 and Experiment 6). It is unclear how ordinal models could be modified to account for both the pure and mixed list result. 
The Psycholinguistic Approach

Gupta (2003) proposed that memory and language processes are controlled by a common underlying system. Gupta noted that new word learning, immediate serial recall and non-word repetition were related abilities that relied on a common system. Gupta's model, which is depicted in Figure 12.1, combines verbal long-term memory and verbal short-term memory in a unitary system.

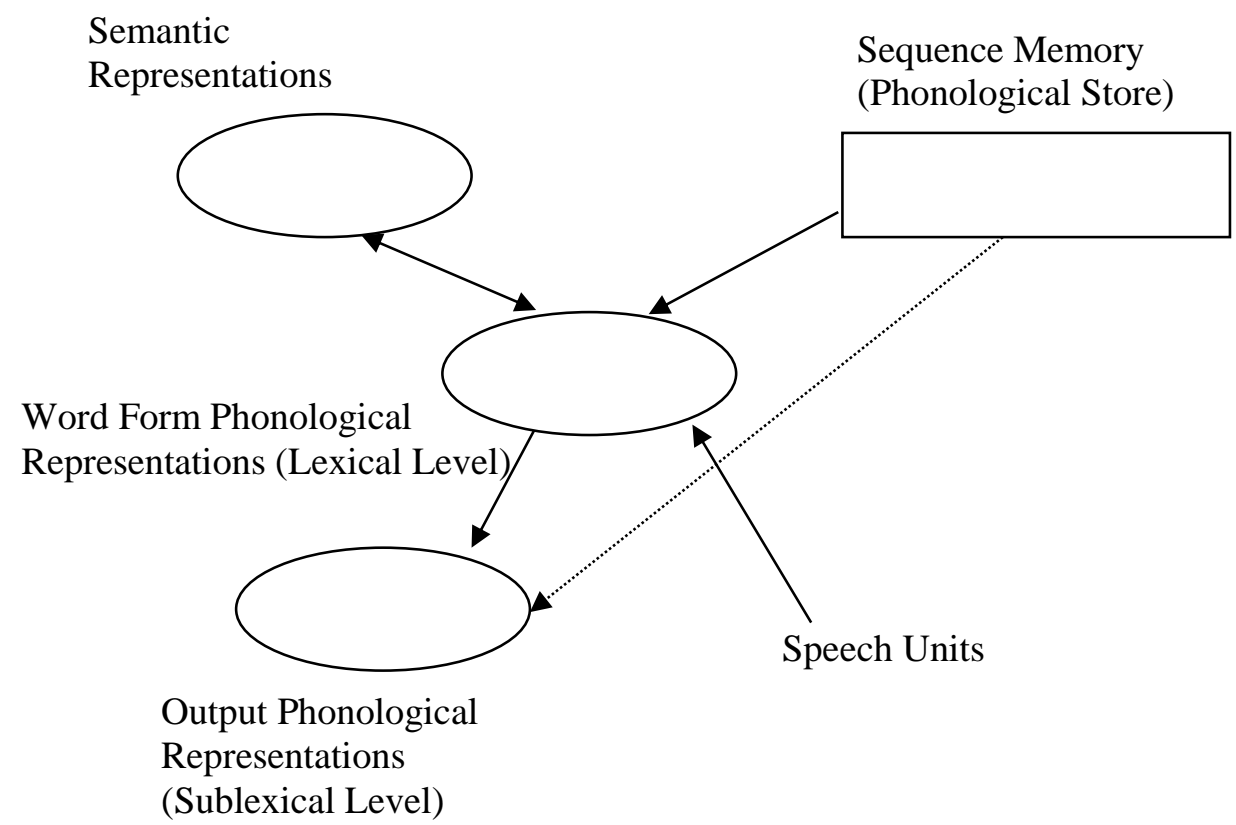

Figure 12.1 Theoretical model of the relationship between ISR, non-word repetition and word learning. The solid lines represent the original model by Gupta (1995), while the broken line between sequence memory and the output phonological representations was theorised in Gupta (2003).

The concept of sequence memory roughly corresponds to the phonological store of the working memory model. In Gupta's model, rather than being a separate store of items, the system is a specialised sequencing mechanism that sets up associations to a pattern of activation in the lexical system. In this way, order is stored as temporary activation across a set of units in long-term memory. In this system, the serial order of word forms is coded via temporary learning in the short-term connection weights between the lexical level and the sequence memory. The lexical level then encodes the serial order of its sublexical level units. Gupta (2003) revised 
the model based on evidence for common effects in short-term memory and linguistic tasks. Specifically, Gupta investigated the relationship between non-word repetition and immediate serial memory and found primacy and recency effects in repetition of polysyllabic non-words, which mimics the pattern of performance in immediate serial recall. This is consistent with a short-term memory system that is common to immediate serial recall and non-word repetition. Further, it suggests that sequence memory has a direct link with sublexical units in verbal long-term memory. In this revision, short-term connections exist directly between sequence memory and the sublexical level. Providing the connections weights have not decayed too much, the sequence of activations can be recalled by sequence memory.

The finding that phonological neighbourhood density influences memory for order can be explained as the pattern of activation representing the order of a list of large neighbourhood words being better specified than the pattern of activation representing the order of small neighbourhood words. This suggests that the shortterm connections between sequence memory and the lexical and sublexical level are stronger for large neighbourhood words. Gupta (2003) gives no indication of the exact mechanisms within each of these levels, although it is evident from the current experiments that lexical neighbours must be able to contribute to the strength of the connection with sequence memory in this model.

Acheson, Hamidi, Binder, and Postle (2011) provided evidence that verbal working memory operates as a subset of language production. They suggest that the phonological store and the phonological loop concepts of short-term memory correspond to the phonological encoding and articulatory planning concepts of speech production. To examine causal evidence for a common underlying system they first identified the neurological areas associated with the phonological encoding and lexical-semantic retrieval aspects of language production using fMRI. They then looked at the effects of targeting these areas using repetitive transcranial magnetic stimulation (rTMS) on a speech production task (non-word reading) and a working memory task (delayed serial recall). TMS can be used to demonstrate causal relationships between behaviour and a stimulated area by temporarily inhibiting neuronal activity in a highly localised area (Rossini \& Rossi, 2007). They found that participants were more likely to omit items during delayed recall when rTMS was applied to the posterior superior temporal gyrus (phonological encoding) but not when 
applied to the middle temporal gyrus (lexical-semantic retrieval). A similar pattern of results was found for non-word reading indicating that working memory and speech production have a similar underlying system. There were no effects observed for item ordering errors. This suggests that neither phonological encoding nor lexical-semantic retrieval is responsible for memory for order. Instead, Acheson and Macdonald (2009b) suggest that order is constrained by motor planning in articulation, a process that occurs later in the production process than examined in Acheson et al., (2011). This is supported in the current series of experiments by the finding that concurrent articulation removes the neighbourhood effect on memory for order. This differs from the explanation traditionally offered for the effects of articulatory suppression, most notably for the removal of the phonological similarity effect under conditions of articulatory suppression for visually, but not for verbally, presented material. Baddeley et al. (1984) suggested that this reflected the phonological nature of verbal working memory, with articulatory suppression preventing visually presented items from being transcribed into an articulatory code. However, Jones, Macken, and Nicholls (2004) ascribed the effect to the rehearsal process and stated that articulatory rehearsal is governed by speech planning processes. This latter explanation is consistent with results from the current series of experiments.

\subsubsection{Conclusions}

Models that use compound chaining mechanisms, such as TODAM can be used to explain the effect of neighbourhood density on memory for order of pure lists, with long-term memory contributing to the convolution (ordering) process during encoding. However, TODAM struggles to explain the pattern of results from mixed lists, unless it is assumed that this effect occurs due to erroneous cueing at recall. This explanation could be further examined by looking at relative rather than absolute performance across mixed lists. The mixed list finding also presents a challenge for positional models, which would predict a saw-tooth serial position curve for these items. Ordinal models fare little better than positional models, with the mechanisms predicting a queue-jumping finding such as that proposed by Roodenrys and his colleague (20002), which was not supported by the current findings. While the pattern of results presents a challenge for all three model types, TODAM comes closest to 
explaining both the mixed list and the pure list finding, without altering the model structure. However, TODAM makes no effort to model the effects of articulatory suppression, and until these mechanisms are specified, alternative approaches to serial order must be sought to explain the effects of articulatory suppression in the current series of experiments.

The findings can be explained by psycholinguistic models, which suggest a common underlying system for memory and speech production processes. This is supported in the current experiments by the removal of the neighbourhood effect on memory for order under conditions of articulatory suppression. Current theoretical development in psycholinguistics has focussed on creating models that can address both basic short-term memory and basic speech production processes. These models are in their infancy and as such a clear understanding of the mechanisms behind the phonological neighbourhood effect on memory for order in mixed and pure lists is unclear. Specification of these mechanisms is necessary to elucidate how this effect occurs within this system.

\subsection{Suggestions for Future Research}

One possible avenue for future investigation is to ascertain whether the findings from Experiment 11 were due to participants not encoding for recall as expected from Duncan and Murdock (2000). One way to examine this would be to vary the number of recall to reconstruction trials in a post cued experiment. If participants are given more recall than reconstruction trials then encoding will be biased towards recall.

Currently, little research exists on participants' ability to self-monitor order errors in serial recall tasks (Acheson \& MacDonald, 2009b). Participants seem capable of identifying and correcting order errors in speech but it is unclear whether this capacity is extended to working memory tasks. In serial recall, this is in part due to task instruction, as participants are not able to correct themselves once they have made an error. However, it is also possible that given no instruction, participants would still avoid self-correction lest this cause further interference or decay of the remaining list items. Acheson and MacDonald (2009b) suggested the use of a confidence scale, to be completed by participants after they complete each trial. This 
would allow an examination of whether order errors in lists of small neighbourhood words are due to item loss for these items, or whether these items are misordered subconsciously within a cognitive store. If participants were confident that the erroneous items were ordered correctly, this would indicate that the order errors occur at a level that is not conscious to participants. If participants indicated that they were not confident with their orderings, this may be indicative of a guessing parameter either due to loss of item information or loss of order information or both.

Investigation into the neural underpinnings of verbal memory is a fertile area for future research. The current thesis pin-points the locus of the neighbourhood effect on memory for order to long-term memory influences occurring prior to redintegration, most likely during rehearsal. However, the possibility remains that the influence of phonological neighbourhood density occurs due to encoding advantages afforded to large neighbourhood words. Marshuetz (2005) notes evidence for neurological systems dedicated to the coding for sequential order, namely prefrontal regions including the dorsolateral and ventrolateral prefrontal cortex. It would be of value to ascertain whether these regions are associated with concurrent activation of the systems dedicated to permanent storage of information, the right superior temporal gyrus (BA 22) and the right middle frontal gyrus (BA 9) (Collette, et al., 2001).

One limitation of the experiments conducted in this body of research is that stimuli were only matched for word frequency and neighbourhood frequency, and, in Experiment 7, word length. It would be important for further studies to match stimuli on concreteness and articulatory duration. This was not done in the current experiments as these values were not available for the stimuli used.

Finally, the conceptions of how words interact within phonological space are constantly evolving. One current notion is Vitevitch's (2008) clustering coefficient. This involves not just a count of how many immediate phonological neighbours a word has but also how many of these words are neighbours with one another. An examination of the phonological neighbourhood effect on serial reconstruction of order that includes altering the definition of phonological neighbourhood density in line with clustering coefficient would provide a richer understanding of how words interact. This would provide a deeper understanding of how activation flows between 
nodes within the lexical network and how this interacts to assist short-term memory for order.

\subsection{Conclusion}

The current series of experiments examined phonological neighbourhood density effects on short-term memory for order. Roodenrys et al. (2002) found a novel effect of neighbourhood density on the pattern of order errors in serial recall. Further, Allen and Hulme (2006) found a small but significant effect of neighbourhood density on the ordered recall of pure lists. The implication of these effects is a long-term memory influence that occurs earlier than that purported by two-stage models of memory. The results of the current thesis indicate that long-term memory exerts an influence on order memory, such that memory for order is more stable for large neighbourhood items than for small neighbourhood items. The results are parsimonious with a long-term memory influence that occurs during the maintenance of items, either pointing to an influence of redintegration during rehearsal or to a psycholinguistic account of memory (i.e., Gupta, 2003). Regardless of whether the effect is due to rehearsal mechanisms or is indicative of a purely psycholinguistic model, it is evident that the influence relies on systems typically associated with speech production, suggesting a confluence between memory and language processes. 


\section{References}

Acheson, D. J., Hamidi, M., Binder, J. R., \& Postle, B. R. (2011). A common neural substrate for language production and verbal working memory. Journal of Cognitive Neuroscience, 23(6), 1358-1367.

Acheson, D. J., \& MacDonald, M. C. (2009a). Twisting tongues and memories: Explorations of the relationship between language production and verbal working memory. Journal of Memory and Language, 60(3), 329-350.

Acheson, D. J., \& MacDonald, M. C. (2009b). Verbal working memory and language production: Common approaches to the serial ordering of verbal information. Psychological Bulletin, 135(1), 50-68.

Albinger, I., Abel, S. \& Huber, W. (2008). Deep dysphasia as a phonetic input deficit: Evidence from a single case. Aphasiology, 22(5), 537-556.

Allen, R., \& Hulme, C. (2006). Speech and language processing mechanisms in verbal serial recall. Journal of Memory and Language, 55, 64-88.

Asch, S. E., \& Ebenholtz, S. M. (1962). The process of free recall: Evidence for nonassociative factors in acquisition and retention. Journal of Psychology, 54(3$31)$.

Atkinson, R. C., \& Shiffrin, R. M. (1968). Human memory: A proposed system and its control processes. In K. W. Spence \& J. T. Spence (Eds.), The psychology of learning and motivation (Vol. 2, pp. 89-195). New York: Academic Press.

Attout, L., Van der Kaa, M., George, M., \& Majerus, S. (2012). Dissociating shortterm memory and language impairment: The importance of item and serial order information. Aphasiology, 26(3-4), 355-382.

Baars, B. J., Motley, M. T., \& MacKay, D. G. (1975). Output editing for lexical status from artificially elicited slips of the tongue. Journal of Verbal Learning and Verbal Behavior, 14, 382-391.

Baayen, R. H., Pipenbrock, R., \& van Rijan, H. (1993). The Celex Lexical Database. Philadelphia: Linguistic data consortium, University of Pennsylvania. 
Baddeley, A. D. (1966a). Short-term memory for word sequences as a function of acoustic, semantic, and formal similarity. Quarterly Journal of Experimental Psychology, 18, 362-365.

Baddeley, A. D. (1966b). The influence of acoustic and semantic similarity on longterm memory for words. Quarterly Journal of Experimental Psychology, 18, 302-309.

Baddeley, A. D. (1986). Working Memory. Oxford: Oxford University Press.

Baddeley, A. D. (1997). Human memory: Theory and practice. Hove, UK: Psychology Press.

Baddeley, A. D. (2000). The episodic buffer: a new component of working memory? Trends in Cognitive Science, 4(11), 417-423.

Baddeley, A. D. (2001). Is working memory still working? American Psychologist, $56,851-864$.

Baddeley, A. D. (2002). Is working memory still working? European Psychologist, 7, 85-97.

Baddeley, A. D., \& Andrade, J. (1994). Reversing the word-length effect: A comment on Caplan, Rochon, and Waters. The Quarterly Journal of Experimental Psychology, 47A, 1047-1054.

Baddeley, A. D., Chincotta, D., Stafford, L., \& Turk, D. (2002). Is the word length effect in STM entirely attributable to output delay? Evidence from serial recognition. The Quarterly Journal of Experimental Psychology Section A, 55(2), 353-369.

Baddeley, A. D., \& Hitch, G. (1974). Working memory. In G. H. Bower (Ed.), The psychology of learning and motivation (Vol. 8, pp. 47-90). New York: Academic Press.

Baddeley, A. D., \& Levy, B. A. (1971). Semantic coding and short-term memory Journal of Experimental Psychology, 89, 132-136. 
Baddeley, A. D., Lewis, V. J., \& Vallar, G. (1984). Exploring the articulatory loop. Quarterly Journal of Experimental Psychology, 36, 233-252.

Baddeley, A. D., Thomson, N., \& Buchanan, M. (1975). Word length and the structure of short-term memory. Journal of Verbal Learning and Verbal Behavior, 14(6), 575-589.

Baddeley, A. D., \& Wilson, B. A. (1985). Phonological coding and short-term memory in patients without speech. Journal of Memory and Language, 24, 490-502.

Baus, C., Costa, A., \& Carreiras, M. (2008). Neighbourhood density and frequency effects in speech production: A case for interactivity. Language and Cognitive Processes, 23(6), 866-888.

Beaman, C. P. (2006). The relationship between absolute and proportion scores of serial order memory: Simulation predictions and empirical data. Psychonomic Bulletin and Review, 13, 92-98.

Besner, D., \& Davelaar, E. (1982). Basic processes in reading: Two phonological codes. Canadian Journal of Psychology, 36, 701-711.

Bjork, E. L., \& Healy, A. F. (1974). Short-term order and item retention. Journal of Verbal Learning and Verbal Behavior, 13, 90-97.

Bock, K., \& Levelt, W. J. M. (1994). Language production: Grammatical encoding. In M. A. Gernsbacher (Ed.), Handbook of Psycholinguistics (pp. 945-984). London: Academic Press.

Bond, Z., S. (1999). Slips of the ear: Errors in the perception of casual conversation. New York: Academic Press.

Broadbent, D. (1957). A mechanical model for human attention and immediate memory. Psychological Review, 64, 205-215.

Broadbent, D. (1958). Perception and Communication. London: Pergamon Press.

Brooks, J. O., \& Watkins, M. J. (1990). Further evidence of the intricacy of memory 
span. Journal of Experimental Psychology: Learning, Memory, and Cognition, $16,1134-1141$.

Brown, G. D. A., Preece, T., \& Hulme, C. (2000). Oscillator-based memory for serial order. Psychological Review, 107, 127-181.

Brown, J. (1958). Some tests of the decay theory of immediate memory. Quarterly Journal of Experimental Psychology, 10, 12-21.

Burgess, N., \& Hitch, G. (1992). Toward a network model of the articulatory loop. Journal of Memory and Language, 31, 429-460.

Burgess, N., \& Hitch, G. (2005). Computation models of working memory: putting long term memory into context. Trends in Cognitive Science, 9, 535-541.

Burgess, N., \& Hitch, G. J. (1999). Memory for serial order: A network model of the phonological loop and its timing. Psychological Review, 106(3), 551-581.

Camos, V., Lagner, P., \& Barrouillet, P. (2009). Two maintenance mechanisms of verbal information in working memory. Journal of Memory and Language, 61(3), 457-469.

Caplan, D., Rochon, E., \& Waters, G. S. (1992). Articulatory and phonological determinants of word length effects in span tasks. The Quarterly Journal of Experimental Psychology Section A: Human Experimental Psychology, 45(2), 177-192.

Caplan, D., \& Waters, G. S. (1995). Aphasic disturbances of syntactic comprehension and working memory capacity. Cognitive Neuropsychology, 12, 637-649.

Chan, K. Y., \& Vitevitch, M. S. (2009). The influence of the phonological neighborhood clustering-coefficient on spoken word recognition. Journal of Experimental Psychology: Human Perception and Performance, 35, 19341949.

Cofer, C. N. (1976). The structure of human memory. San Francisco: W. H. Freeman \& Co Ltd. 
Cole, R. A., Jakimik, J., \& Cooper, W. E. (1978). Perceptibility of phonetic features in fluent speech. Journal of the Acoustical Society of America, 64, 44-56.

Collette, F., Van der Linden, M., Delfiore, G., Degueldre, C., Luxen, A., \& Salmon, E. (2001). The functional anatomy of inhibition processes investigated with the Hayling task. Neuroimage, 14(2), 258-267.

Coltheart, M., Davelaar, E., Jonasson, J. T., \& Besner, D. (Eds.). (1977). Access to the internal lexicon. Hillsdale: Lawrence Erlbaum Associates.

Coltheart, M., Rastle, K., Perry, C., Langdon, R., \& Ziegler, J. (2001). A dual route cascaded model of visual word recognition and reading aloud. Psychological Review, 108, 201-256.

Conrad, R. (1960). Serial order intrusions in immediate memory. British Journal of Psychology, 51, 45-48.

Conrad, R. (1964). Acoustic confusions in immediate memory. British Journal of Psychology, 55, 75-84.

Conrad, R. (1965). Order error in immediate recall of sequences. Journal of Verbal Learning and Verbal Behavior, 4(3), 161-169.

Cowan, N. (1984). On short and long auditory stores. Psychological Bulletin, 96(2), 341-370.

Cowan, N. (1988). Evolving conceptions of memory storage, selective attention, and their mutual constraints within the human information-processing system. Psychological Bulletin, 104(2), 163-191.

Cowan, N. (1992). Verbal memory span and the timing of spoken recall. Journal of Memory and Language, 31, 668-684.

Cowan, N. (1995). Attention and memory: An integrated framework. New York: Oxford University Press.

Cowan, N. (1999). An embedded-process model of working memory. In A. Miyake \& P. Shah (Eds.), Models of working memory: Mechanisms of active 
maintenance and executive control (pp. 62-101): Cambridge University Press.

Cowan, N., Day, L., Saults, J. S., Keller, T. A., Johnson, T., \& Flores, L. (1992). The role of verbal output time in the effects of word length on immediate memory. Journal of Memory and Language, 31, 1-17.

Dell, G. S. (1986). A spreading-activation theory of retrieval in sentence production. Psychological Review, 92, 283-321.

Dell, G. S. (1988). The retrieval of phonological forms in production: Tests of predictions from a connectionist model. Journal of Memory and Language, $27,124-142$

Dell, G. S. (1999). Commentary: counting, connectionism, and lexical representation. In M. B. Broe \& J. Breckenridge Pierrehumbert (Eds.), Acquisition and the lexicon (pp. 335-348). Cambridge: Cambridge University Press.

Dell, G. S., Burger, L. K., \& Svec, W. R. (1997). Language production and serial order: A functional analysis and a model. Psychological Review, 85, 123-147.

Dell, G. S., \& Gordon, J. K. (2003). Learning to divide the labor: An account of deficits in light and heavy verb production. Cognitive Science, 27, 1-40.

Dell, G. S., \& O'Seaghdha, P. G. (1992). Stages of lexical access in language production. Cognition, 42, 287-314.

Dell, G. S., Schwartz, M. F., Martin, N., Saffran, E. M., \& Gagnon, D. A. (1997). Lexical access in aphasic and nonaphasic speakers. Psychological Review, $104,801-838$.

DeLosh, E. L., \& McDaniel, M. A. (1996). The role of order information in free recall: Application to the word frequency effect. Journal of Experimental Psychology: Learning, Memory, and Cognition, 22, 1136-1146.

Dirks, D. D., Takayanagi, S., Moshfegh, A., Noffsinger, P. D., \& Fausti, S. A. (2001). Examination of the neighborhood activation theory in normal and hearingimpaired listeners. Ear \& Hearing, 22, 1-13. 
Donaldson, W., \& Glathe, H. (1969). Recognition memory for item and order information. Journal of Experimental Psychology, 82, 557-560.

Dosher, B. A., \& Ma, J. J. (1998). Output loss or rehearsal loop? Output-time versus pronunciation-time limits in immediate recall for forgetting-matched materials. Journal of Experimental Psychology: Learning, Memory, and Cognition, 24, 316-335.

Duncan, M., \& Murdock, B. B. (2000). Recognition and recall with precuing and postcuing. Journal of Memory and Language, 42, 301-313.

Ebbinghaus, H. (1913). Memory: A contribution to experimental psychology (H. A. Ruger \& C. E. Bussenius, Trans.). New York: Teachers College, Columbia University.

Ellis, A. W. (1980). Errors in speech and short-term memory: The effects of phonological similarity and syllable position. Journal of Verbal Learning and Verbal Behavior, 19, 624-634.

Ellis, N., \& Sinclair, S. (1996). Working memory in the acquisition of vocabulary and syntax: Putting language in good order. The Quarterly Journal of Experimental Psychology Section A, 49, 234-250.

Ellis, N. C., \& Hennelly, R. A. (1980). A bilingual word-length effect: Implications for intelligence testing and the relative ease of mental calculation in Welsh and English. British Journal of Psychology, 71, 43-51.

Ericsson, K. A., \& Kintsch, W. (1995). Long-term working memory. Psychological Review, 102, 211-245.

Estes, W. K. (1972). An associative basis for coding and organization in memory. In A. W. Melton \& E. Martin (Eds.), Coding processes in human memory (pp. 161-190). Washington, DC: Winston.

Forster, K. I., \& Chambers, S. (1973). Lexical access and naming time. Journal of Verbal Learning and Verbal Behavior, 12, 627-635.

Fromkin, V. A. (1971). The non-anomalous nature of anomalous utterances. 
Language, 47, 27-52.

Gathercole, S. E., \& Baddeley, A. D. (1989). Evaluation of the role of phonological STM in the development of vocabulary in children: A longitudinal study. Journal of Memory and Language, 28, 200-213.

Gathercole, S. E., Frankish, C. R., Pickering, S., J., \& Peaker, S. J. (1999).

Phonotactic influences on short-term memory. Journal of Experimental Psychology: Learning, Memory, and Cognition, 25, 84-95.

Gathercole, S. E., Service, E., Hitch, G., Adams, A. M., \& Martin, A. J. (1999). Phonological short-term memory and vocabulary development: Further evidence on the nature of the relationship. Applied Cognitive Psychology, 13, $65-77$.

Geffen, G., \& Luszcz, M. A. (1983). Are the spoken durations of rare words longer than those of common words? Memory \& Cognition, 11, 13-15.

Glasspool, D. W. (1995). Competitive queuing and the articulatory loop. In J. Levy, D. Bairaktaris, J. A. Bullinaria \& P. Cairns (Eds.), Connectionist models of memory and language. London: UCL Press.

Glenberg, A. M., \& Swanson, N. (1986). A temporal distinctiveness theory of recency and modality effects. Journal of Experimental Psychology: Learning, Memory, and Cognition, 12, 3-24.

Goldinger, S. D., Azuma, T., Abramson, M., \& Jain, P. (1997). Open wide and say "blah!" Attentional dynamics of delayed naming. Journal of Memory and Language, 37, 190-216.

Goldinger, S. D., Luce, P. A., \& Pisoni, D. B. (1989). Priming lexical neighbors of spoken words: Effects of competition and inhibition. Journal of Memory and Language, 28, 501-518.

Gregg, V. H., Freedman, C. M., \& Smith, D. K. (1989). Word frequency, articulatory suppression and memory span. British Journal of Psychology, 80(3), 363.

Grossberg, S. (1978). Behavioral contrast in short term memory: Serial binary 
memory models or parallel continuous memory models? Journal of Mathematical Psychology, 17, 199-219.

Grossberg, S., Boardman, I., \& Cohen, M. (1997). Neural dynamics of variable-rate speech categorization. Journal of Experimental Psychology: Human Perception and Performance, 23, 483-503.

Gupta, P. (2003). Examining the relationship between word learning, nonword repetition, and immediate serial recall in adults. Quarterly Journal of Experimental Psychology, 56, 1213-1236.

Gupta, P., \& MacWhinney, B. (1997). Vocabulary acquisition and verbal short-term memory: Computational and neural bases. Brain and Language, 59(267-333).

Hartley, T., \& Houghton, G. (1996). A linguistically-constrained model of short-term memory for nonwords. Journal of Memory and Language, 53, 1-31.

Healy, A. F. (1974). Separating item from order information in short-term memory. Journal of Verbal Learning and Verbal Behavior, 13, 644-655.

Hebb, D. O. (1961). The organization of behavior. New York: John Wiley \& Sons, Inc.

Henson, R. N. A. (1996). Short-term memory for serial order. Unpublished doctoral thesis, University of Cambridge.

Henson, R. N. A. (1998a). Short-term memory for serial order: the Start-End Model. Cognitive Psychology, 36, 73-137.

Henson, R. N. A. (1999). Coding position in short-term memory. International Journal of Psychology, 34, 403-409.

Henson, R. N. A., \& Burgess, N. (1997). Representations of serial order. In J. A. Bullinaria, D. W. Glasspool \& G. Houghton (Eds.), 4th Neural Computation and Psychology Workshop (pp. 283-300). London: Springer.

Henson, R. N. A., Hartley, T., Burgess, N., Hitch, G., \& Flude, F. (2003). Selective interference with verbal short-term memory for serial order information: A 
new paradigm and tests of a timing-signal hypothesis. Quarterly Journal of Experimental Psychology Section A, 56(8), 1307-1334.

Henson, R. N. A., Norris, D. G., Page, M. P. A., \& Baddeley, A. D. (1996). Unchained memory: Error patterns rule out chaining models of immediate serial recall. Quarterly Journal of Experimental Psychology, 49A, 80-115.

Hitch, G., Fastame, M. C., \& Flude, B. (2005). How is the serial order of a verbal sequence coded? Some comparisons between models. Memory, 3-4, 247-258.

Hoover, J. R., Storkel, H. L., \& Hogan, T. P. (2010). A cross-sectional comparison of the effects of phonotactic probability and neighborhood density on word learning by preschool children. Journal of Memory and Language, 63, 100116.

Houghton, G. (1990). The problem of serial order: A neural network model of sequence learning and recall. In R. Dale, C. Mellish \& M. Zock (Eds.), Current research in natural language generation (pp. 287-319). London: Academic Press.

Hulme, C., Maughan, S., \& Brown, G. D. A. (1991). Memory for familiar and unfamiliar: Evidence for a long-term memory contribution to short-term memory span. Journal of Memory and Language, 30, 685-701.

Hulme, C., Newton, P., Cowan, N., Stuart, G., \& Brown, G. D. A. (1999). Think before you speak: pause, memory search and trace redintegration processes in verbal memory span. . Journal of Experimental Psychology: Learning, Memory, and Cognition, 25, 447-463.

Hulme, C., Roodenrys, S., Brown, G. D. A., \& Mercer, R. (1995). The role of longterm memory mechanisms in memory span. British Journal of Psychology, 86, $527-536$.

Hulme, C., Roodenrys, S., Schweickert, R., Brown, G. D. A., Martin, S., \& Stuart, G. (1997). Word-frequency effects on short-term memory tasks: Evidence for a redintegration process in immediate serial recall. Journal of Experimental Psychology: Learning, Memory, and Cognition, 23, 1217-1232. 
Hulme, C., Stuart, G., Brown, G. D. A., \& Morin, C. (2003). High- and lowfrequency words are recalled equally well in alternating lists: Evidence for associative effects in serial recall. Journal of Memory and Language, 49(4), 500-518.

Hulme, C., Surprenant, A. M., Bireta, T. J., Stuart, G., \& Neath, I. (2004). Abolishing the word length effect. Journal of Experimental Psychology: Learning, Memory, and Cognition, 30(1), 98-106.

Jalbert, A., Neath, I., Bireta, T. J., \& Surprenant, A. M. (2011). When does length cause the word length effect? Journal of Experimental Psychology: Learning, Memory, and Cognition, 37(2), 338-353.

James, L. E., \& Burke, D. M. (2000). Phonological priming effects on word retrieval and tip-of-the-tongue experiences in young and older adults. Journal of Experimental Psychology: Learning, Memory, and Cognition, 26, 1378-1391.

James, W. (1890). Principles of Psychology (Vol. 1): Henry Hold and Co, Inc.

Jescheniak, J. D., \& Levelt, W. J. M. (1994). Word frequency effects in speech production: Retrieval of syntactic information and of phonological form. Journal of Experimental Psychology: Learning, Memory, and Cognition, 20, 824-843.

Jones, D. M., Macken, W. J., \& Nicholls, A. P. (2004). The phonological store of working memory: Is it phonological and is it a store? Journal of Experimental Psychology: Learning, Memory, and Cognition, 30(3), 656-674.

Jones, G. V. (1989). Back to Woodworth: Role of interlopers in the tip of the tongue phenomenon. Memory \& Cognition, 17, 69-76.

Kesner, R. P., Hopkins, R. O., \& Fineman, B. (1994). Item and order dissociation in humans with prefrontal cortex damage. Neuropsychologia, 32(881-891).

Kidd, G. R., \& Greenwalk, A. G. (1988). Attention, rehearsal, and memory for serial order. American Journal of Psychology, 101, 259-279.

Kucera, H., \& Francis, W. N. (1967). Computational analysis of present-day 
American English. Providence, Rhode Island: Brown University Press.

Landauer, T. K., \& Streeter, L. A. (1973). Structural differences between common and rare words: Failure of equivalence assumptions for theories of word recognition. Journal of Verbal Learning and Verbal Behavior, 12, 119-131.

Lashley, K. S. (1951). The problem of serial order in behavior. In L. A. Jeffress (Ed.), Cerebral mechanisms in behavior. New York: Wiley.

Leach, L., \& Samuel, A. (2007). Lexical configuration and lexical engagement: When adults learn new words. Cognitive Psychology, 55(4), 306-353.

Leclercq, A. L., \& Majerus, S. (2010). Serial-order short-term memory predicts vocabulary development: Evidence from a longitudinal study. Developmental Psychology, 46(2), 417-427.

Lee, C. L., \& Estes, W. K. (1977). Order and position in primary memory for letter strings. Journal of Verbal Learning and Verbal Memory, 16, 395-418.

Lee, C. L., \& Estes, W. K. (1981). Item and order information in short-term memory: Evidence for multilevel perturbation processes. Journal of Experimental Psychology: Human Learning and Memory, 7, 149-169.

Levelt, W. J. M. (1989). Speaking: From intention to articulation. Cambridge, MA: MIT Press.

Levelt, W. J. M. (1999). Models of word production. Trends in Cognitive Science, 3(6), 223-232.

Levelt, W. J. M., Roelofs, A., \& Meyer, A. S. (1999). A theory of lexical access in speed production. Behavioral and Brain Sciences, 22, 1-75.

Lewandowsky, S., \& Murdock, B. B. (1989). Memory for serial order. Psychological Review, 96, 25-57.

Luce, P. A., Goldinger, S. D., Auer, E. T., \& Vitevitch, M. S. (2000). Phonetic priming, neighborhood activation, and PARSYN. Perception and Psychophysics, 62, 615-625. 
Luce, P. A., \& Pisoni, D. B. (1998). Recognizing spoken words: The neighborhood activation model. Ear \& Hearing, 19, 1-36.

Luce, P. A., Pisoni, D. B., \& Goldinger, S. D. (1990). Similarity neighborhoods of spoken words. In G. Altmann (Ed.), Cognitive models of speech perception: Psycholinguistic and computational perspectives. Cambridge: MIT Press.

Majerus, S. (2009). Verbal short-term memory and temporary activation of language representations: the importance of distinguishing item and order information. In A. Thorn \& M. P. A. Page (Eds.), Interactions between short-term and long-term memory in the verbal domain. London: Psychology Press.

Majerus, S., \& D'Argembeau, A. (2011). Verbal short-term memory reflects the organization of long-term memory. Further evidence from short-term memory for emotional words. Journal of Memory and Language, 64, 181-197.

Majerus, S., Poncelet, M., Elsen, B., \& Van der Linden, M. (2006). Exploring the relationship between new word learning and short-term memory for serial order recall, item recall, and item recognition. European Journal of Cognitive Psychology, 18, 848-873.

Majerus, S., Poncelet, M., Greffe, C., \& Van der Linden, M. (2006). Relations between vocabulary development and verbal short-term memory: The relative importance of short-term memory for serial order and item information. Journal of Experimental Child Psychology, 93, 95-119.

Manelis, J. (1977). Frequency and meaningfulness in tachistoscopic word perception. American Journal of Psychology, 99(269-280).

Marshuetz, C. (2005). Order information in working memory: an integrative review of evidence from brain and behavior. Psychological Bulletin, 131(3), 323-339.

Marslen-Wilson, W. D., \& Welsh, A. (1978). Processing interactions and lexical access during word-recognition in continuous speech. Cognitive Psychology, $10,29-63$.

Martin, N., \& Saffran, E. M. (1997). Language and auditory-verbal short-term 
memory impairments: Evidence for common underlying processes. Cognitive Neuropsychology, 14(641-682).

Martin, N., Saffran, E. M., \& Dell, G. S. (1996). Recovery in deep dysphasia: Evidence for a relationship between auditory-verbal STM capacity and lexical errors in repetition. Brain and Language, 52, 83-113.

Martin, R. C. (2007). Semantic short-term memory, language processing, and inhibition. In A. S. Meyer, L. R. Wheeldon \& A. Knott (Eds.), Automaticity and control in language processing (pp. 161-191). Hove: Psychology Press.

Martin, R. C., Lesch, M. F., \& Bartha, M. C. (1999). Independence of input and output phonology in word processing and short-term memory. Journal of Memory and Language, 41, 3-29.

McClelland, J. L., \& Elman, J. L. (1986). The TRACE model of speech perception. Cognitive Psychology, 18, 1-86.

McDaniel, M. A., DeLosh, E. L., \& Merritt, P. S. (2000). Order information and retrieval distinctiveness: Recall of common versus bizarre material. Journal of Experimental Psychology: Learning, Memory, and Cognition, 26(4), 10451056.

McElree, B., \& Dosher, B. A. (1993). Serial retrieval processes in the recovery of order information. Journal of Experimental Psychology: General, 122, 291315.

Melton, A. W. (1963). Implications of short-term memory for a general theory of memory. Journal of Verbal Learning and Verbal Behavior, 2, 1-21.

Metsala, J. L. (1999). The development of phonemic awareness in reading-disabled children. Applied Psycholinguistics, 20(1), 149-158.

Meyer, A. S., \& Bock, K. (1992). The tip-of-the-tongue phenomenon: Blocking or partial activation. Memory \& Cognition, 20, 715-726.

Mikkulainen, R. (1997). Dyslexic and category-specific aphasic impairments in a selforganizing feature map model of the lexicon. Brain and Language, 59(2), 334- 
366.

Miller, G. A. (1956). The magical number seven, plus or minus two: Some limits on our capacity for processing information. The Psychological Review, 63, 81-97.

Miller, L. M., \& Roodenrys, S. (2012). Serial recall, word frequency, and mixed lists: The influence of item arrangement. Journal of Experimental Psychology: Learning, Memory, and Cognition, Advanced online publication.

Miller, L. M., \& Roodenrys, S. J. (2009). The interaction of word frequency and concreteness in immediate serial recall. Memory \& Cognition, 37(6), 850-865.

Milner, B. (1968). Disorders of memory after brain lesions in man. Preface: Materialspecific and generalized memory loss. Neuropsychologia, 6, 175-179.

Monnier, C., \& Syssau, A. (2008). Semantic contribution to verbal short-term memory: Are pleasant words easier to remember than neutral words in serial recall and serial recognition? Memory \& Cognition, 36, 35-42.

Morin, C., Poirier, M., Fortin, C., \& Hulme, C. (2006). Word frequency and the mixed-list paradox in immediate and delayed serial recall. Psychonomic Bulletin and Review, 13(4), 724-729.

Mueller, S. T., \& Krawitz, A. (2009). Reconsidering the two-second decay hypothesis in verbal working memory. Journal of Mathematical Psychology, 53, 14-25.

Murdock, B. B. (1967). Transpositions in short-term memory. Journal of Experimental Psychology, 74(1), 137-143.

Murdock, B. B. (1976). Item and order information in short-term memory. Journal of Experimental Psychology: General, 105, 191-216.

Murdock, B. B. (1982). A theory for the storage and retrieval of item and associative information. Psychological Review, 89, 609-626.

Murdock, B. B. (1993). TODAM2: A models for the storage and retrieval of item, associative, and serial-order information. Psychological Review, 100, 183-203.

Murdock, B. B. (1997). Context and mediators in a theory of distributed associative 
memory (TODAM2). Psychological Review, 104, 839-862.

Murdock, B. B., \& vom Saal, W. (1967). Transpositions in short-term memory. Journal of Experimental Psychology, 74, 137-143.

Murray, D. J. (1968). Articulation and acoustic confusability in short-term memory. Journal of Experimental Psychology, 78(4), 679-684.

Nairne, J. S. (1990). A feature model of immediate memory. Memory \& Cognition, $18,251-269$.

Nairne, J. S. (2002). Remembering over the short-term: The case against the standard model. Annual Review of Psychology, 53, 53-81.

Nairne, J. S., \& Kelley, M. R. (2004). Separating item and order information through process dissociation. Journal of Memory and Language, 50, 113-133.

Nairne, J. S., \& Neath, I. (2001). Long-term memory span. Behavioral and Brain Sciences, 24, 134-135.

Neath, I. (1997). Modality, concreteness, and set-size effects in a free reconstruction of order task. Memory \& Cognition, 25, 256-263.

Neath, I. (1999). Modelling the disruptive effects of irrelevant speech on order information. International Journal of Psychology, 35, 410-418.

Neath, I. (2006). A SIMPLE account of Baddeley \& Scott (1971). Memory Lab Technical Report 2006-01: Memorial University of Newfoundland.

Neath, I., \& Nairne, J. S. (1995). Word-length effects in immediate memory: Overwriting trace decay theory. Psychonomic Bulletin and Review, 2, 429441.

Newman, R. S., \& German, D. J. (2002). Effects of lexical factors on lexical access among typical language-learning children and children with word-finding difficulties. Language and Speech, 45(3), 285-317.

Newman, R. S., Sawusch, J. R., \& Luce, P. A. (2005). Do post-onset segments define a lexical neighborhood? Memory \& Cognition, 33(6), 941-960. 
O'Grady, W. D. (2005). How children learn language. Cambridge, UK: Cambridge University Press.

Page, M. P. A., \& Norris, D. (1998). The primacy model: A new model of immediate serial recall. Psychological Review, 105(4), 761-781.

Patterson, K., Graham, N., \& Hodges, J. R. (1994). The impact of semantic memory loss on phonological representations. Journal of Cognitive Neuroscience, 6, $57-69$.

Paulesu, E., Frith, C. D., \& Frackowiak, R. S. J. (1993). The neural correlates of the verbal component of Working Memory. Nature, 362, 342-345.

Peereman, R., \& Content, A. (1997). Orthographic and phonological neighborhoods in naming: Not all neighbors are equally influenced in orthographic space. Journal of Memory and Language, 37, 382-421.

Perez, T., Majerus, S., \& Poncelet, M. (2012). The contribution of short-term memory for serial order to early reading acquisition: Evidence from a longitudinal study. Journal of Experimental Child Psychology, 111(4), 708-723.

Peterson, L. R., \& Peterson, M. J. (1959). Short-term retention of individual verbal items. Journal of Experimental Psychology, 58, 193-198.

Poirier, M., \& Saint-Aubin, J. (1996). Immediate serial recall, word frequency, item identity and item position. Canadian journal of Psychology, 50, 408-412.

Rapp, B., \& Goldrick, M. (2000). Discreteness and interactivity in spoken word production. Psychological Review, 107, 460-499.

Roodenrys, S., \& Hinton, M. (2002). Sublexical or lexical effects on serial recall of nonwords? Journal of Experimental Psychology: Learning, Memory, and Cognition, 28, 29-33.

Roodenrys, S., Hulme, C., Alban, J., Ellis, A. W., \& Brown, G. D. A. (1994). Effects of word frequency and age of acquisition on short-term memory. Memory \& Cognition, 22, 698-701. 
Roodenrys, S., Hulme, C., Lethbridge, A., Hinton, M., \& Nimmo, L. M. (2002). Word-frequency and phonological-neighborhood effects on verbal short-term memory. Journal of Experimental Psychology: Learning, Memory, and Cognition, 28(6), 1019-1034.

Roodenrys, S., \& Stokes, J. (2001). Serial recall and nonword repetition in reading disabled children. Reading and Writing, 14, 379-394.

Roodenrys, S. J. (2009). Explaining phonological neighbourhood effects in short-term memory. In A. Thorn \& M. P. A. Page (Eds.), Interactions between short-term and long-term memory in the verbal domain (pp. 177-197). Hove: Psychology press.

Rossini, P. M., \& Rossi, S. (2007). Transcranial magnetic stimulation: Diagnostic, therapeutic, and research potential. Neurology, 68(7), 484-488.

Rundus, D. (1971). Analysis of rehearsal processes in free recall. Journal of Experimental Psychology, 89, 63-77.

Saint-Aubin, J., \& Poirier, M. (1999a). Semantic Similarity and Immediate Serial Recall: Is There a Detrimental Effect on Order Information? Quarterly Journal of Experimental Psychology: Section A, 52(2), 367-394.

Saint-Aubin, J., \& Poirier, M. (1999b). The influence of long-term memory factors on immediate serial recall: An item and order analysis. International Journal of Psychology, 34, 347-352.

Saint-Aubin, J., \& Poirier, M. (2000). Immediate serial recall of words and nonwords: Tests of the retrieval based hypothesis. Psychonomic Bulletin and Review, 7, 332-340.

Scarborough, D. L., Cortese, C., \& Scarborough, H. (1977). Frequency and repetition effects in lexical memory. Journal of Experimental Psychology: Human Perception and Performance, 3, 1-17.

Schweickert, R. (1993). A multinomial processing tree model for degradation and redintegration in immediate recall. Memory \& Cognition, 21, 168-175. 
Schweickert, R., \& Boruff, B. (1986). Short-term memory capacity: magic number or magic spell? Journal of Experimental Psychology: Learning, Memory, and Cognition, 12(3), 419-425.

Schweickert, R., Chen, S., \& Poirier, M. (1999). Redintegration and the useful lifetime of the verbal memory representation. International Journal of Psychology, 34(5/6), 447-453.

Schweickert, R., Guentert, L., \& Hersberger, L. (1990). Phonological similarity, pronunciation rate, and memory span. Psychological Science, 1, 74-77.

Serra, M., \& Nairne, J. S. (1993). Design controversies and the generation effect: Support for an item-order hypothesis. Memory \& Cognition, 21, 34-40.

Serra, M., \& Nairne, J. S. (2000). Part-set cuing of order information: Implications for associative theories of serial order memory. Memory \& Cognition, 28(5), 847855.

Service, E. (1998). The effect of word length on immediate serial recall depends on phonological complexity, not articulatory duration. The Quarterly Journal of Experimental Psychology Section A: Human Experimental Psychology, 51(2), 283-304.

Sevald, C. A., \& Dell, G. S. (1994). The sequential cuing effect in speech production. Cognition, 53, 91-127.

Shattuck-Hufnagel, S. (1979). Speech errors as evidence for a serial ordering mechanism in sentence production. In W. E. Cooper \& E. C. T. Walker (Eds.), Sentence processing: Psycholinguistic studies presented to Merrill Garrett (pp. 295-342). Hillsdale: Erlbaum.

Smith, E. E., \& Jonides, J. (1999). Storage and executive processes in the frontal lobes. Science, 283, 1657-1661.

Standing, L., Bond, B., Smith, P., \& Isley, C. (1980). Is the immediate memory span determined by subvocalization rate? British Journal of Psychology, 71, 525539. 
Sternberg, S. (1966). High-speed scanning in human memory. Science, 153, 652-654.

Sternberg, S. (1967). Two operations in character recognition: Some evidence from reaction-time measurements. Perception and Psychophysics, 2, 45-53.

Storkel, H. L., Armbruster, J., \& Hogan, T. P. (2006). Differentiating phonotactic probability and neighborhood density in adult word learning. Journal of Speech Language Hearing Research, 49, 1175-1192.

Stuart, G., \& Hulme, C. (2000). The effects of word co-occurrence on short-term memory: Associative links in long-term memory affect short-term memory performance. Journal of Experimental Psychology: Learning, Memory, and Cognition, 26, 796-802.

Sumby, W. H. (1963). Word frequency and serial position effects. Journal of Verbal Learning and Verbal Behavior, 1, 443-450.

Surprenant, A. M., \& Neath, I. (2009). The nine lives of short-term memory. In A. Thorn \& M. P. A. Page (Eds.), Interactions between Short-term and Longterm memory in the Verbal Domain (pp. 16-43). Hove, UK: Psychology Press.

Swan, D. M., \& Goswami, U. (1997). Phonological awareness deficits in developmental dyslexia and the phonological representations hypothesis. Journal of Experimental Child Psychology, 66, 18-41.

Tan, L., \& Ward, G. (2000). A recency-based account of the primacy effect in free recall. Journal of Experimental Psychology: Learning, Memory, and Cognition, 26(6), 1589-1625.

Thomson, J., Richardson, U., \& Goswami, U. (2005). The role of similarity neighborhoods in children's short-term memory: comparing typicallydeveloping children with children with dyslexia. Memory \& Cognition, 33, 1210-1219.

Thorn, A. S. C., \& Frankish, C. R. (2005). Long-term knowledge effects on serial recall of nonwords are not exclusively lexical. Journal of Experimental Psychology: Learning, Memory, and Cognition, 31(4), 729-735. 
Thorn, A. S. C., Frankish, C. R., \& Gathercole, S. E. (2009). The influence of longterm knowledge on short-term memory: Evidence for multiple mechanisms. In A. S. C. Thorn \& C. R. Frankish (Eds.), Interactions between short-term and long-term memory in the verbal domain (pp. 199-219). Hove: Psychology Press.

Thorn, A. S. C., Gathercole, S. E., \& Frankish, C. R. (2002). Language familiarity effects in short-term memory: the role of output delay and long-term knowledge. The Quarterly Journal of Experimental Psychology Section A, 55(4), 1363-1383.

Tulving, E. (2002). Episodic memory: From mind to brain. Annual Review of Psychology, 53, 1-25.

Turner, J. E., Henry, L. A., Smith, P. T., \& Brown, P. A. (2004). Redintegration and lexicality effects in children: Do they depend upon the demands of the memory task? Memory \& Cognition, 32(3), 501-510.

Vitevitch, M. S. (1997). The neighborhood characteristics of malapropisms. Language and Speech, 40, 211-228.

Vitevitch, M. S. (2002). Naturalistic and experimental analyses of word frequency and neighborhood density effects in slips of the ear. Language and Speech, 45(4), 407-434.

Vitevitch, M. S. (2008). What can graph theory tell us about word learning and lexical retrieval? Journal of Speech Language Hearing Research, 51, 408-422.

Vitevitch, M. S., \& Luce, P. A. (1998). When words compete: Levels of processing in spoken word perception. Psychological Science, 9, 325-329.

Vitevitch, M. S., \& Luce, P. A. (1999). Probabilistic phonotactics and spoken word recognition. Journal of Memory and Language, 40, 374-408.

Vitevitch, M. S., Luce, P. A., Charles-Luce, J., \& Kemmerer, D. (1997). Phonotactics and syllable stress: Implications for the processing of spoken nonsense words. Language and Speech, 40, 47-62. 
Vitevitch, M. S., Luce, P. A., Pisoni, D. B., \& Auer, E. T. (1999). Phonotactics, neighborhood activation and lexical access for spoken words. Brain and Language, 68, 306-311.

Vitevitch, M. S., \& Rodriguez, E. (2005). Neighborhood density effects in spoken word recognition in Spanish. Journal of Multilingual Communication Disorders, 3, 64-73.

Vitevitch, M. S., \& Stamer, M. K. (2006). The curious case of competition in Spanish speech production. Language \& Cognitive Processes, 21, 760-770.

Walker, I., \& Hulme, C. (1999). Concrete words are easier to recall than abstract words: Evidence for a semantic contribution to short-term serial recall. Journal of Experimental Psychology: Learning, Memory, and Cognition, 25, 1256-1271.

Watkins, M. J. (1977). The intricacy of memory span. Memory \& Cognition, 5, 529534.

Watkins, O. C., \& Watkins, M. J. (1977). Serial recall and the modality effect: Effects of word frequency. Journal of Experimental Psychology: Human Learning and Memory, 3, 712-718.

Wechsler, D. (1958). The Measurement and Appraisal of Adult Intelligence (fourth ed.) Baltimore (MD): Williams \& Watkins.

Whiteman, H. L., Nairne, J. S., \& Serra, M. (1994). Recognition and recall-like processes in the long-term reconstruction of order. Memory, 2, 275-294.

Wickelgren, W. A. (1966). Associative intrusions in short-term recall. Journal of Experimental Psychology, 72, 853-858.

Woodward, A. J., Macken, W. J., \& Jones, D. M. (2008). Linguistic familiarity in short-term memory: A role for (co-)articulatory fluency? Journal of Memory and Language, 58, 48-65.

Yates, M. (2005). Phonological neighbors speed visual word processing: Evidence from multiple tasks. Journal of Experimental Psychology: Learning, Memory, 
and Cognition, 31, 1385-1397.

Yates, M., Locker, L., \& Simpson, G. B. (2004). The influence of phonological neighborhood on visual word perception. Psychological Bulletin and Review, $11,452-457$.

Ziegler, J., Muneaux, M., \& Grainger, J. (2003). Neighborhood effects in auditory word recognition: Phonological competition and orthographic facilitation. Journal of Memory and Language, 48, 779-793. 
Appendix A

Stimuli Used in the Phase 1 of Experiments

\begin{tabular}{|c|c|c|c|c|}
\hline \multicolumn{2}{|c|}{ Large neighbourhood } & \multicolumn{3}{|c|}{ Small neighbourhood } \\
\hline $\begin{array}{c}\text { bark } \\
\text { bead } \\
\text { bin } \\
\text { boot } \\
\text { buzz } \\
\text { cone } \\
\text { cop } \\
\text { cork } \\
\text { cull } \\
\text { duck } \\
\text { fade } \\
\text { fizz } \\
\text { ham } \\
\text { haze } \\
\text { hike } \\
\text { hood } \\
\text { kite } \\
\text { lag } \\
\text { lard } \\
\text { lice } \\
\text { lick } \\
\text { maim } \\
\text { meek } \\
\text { mole }\end{array}$ & $\begin{array}{c}\text { node } \\
\text { peach } \\
\text { pearl } \\
\text { pod } \\
\text { poise } \\
\text { poke } \\
\text { pun } \\
\text { rake } \\
\text { rim } \\
\text { ripe } \\
\text { robe } \\
\text { sane } \\
\text { sap } \\
\text { shack } \\
\text { siege } \\
\text { sock } \\
\text { tan } \\
\text { tart } \\
\text { thorn } \\
\text { tile } \\
\text { vine } \\
\text { weep } \\
\text { weird }\end{array}$ & $\begin{array}{c}\text { badge } \\
\text { beige } \\
\text { carve } \\
\text { chase } \\
\text { chef } \\
\text { chime } \\
\text { churn } \\
\text { couch } \\
\text { dab } \\
\text { dodge } \\
\text { fang } \\
\text { fog } \\
\text { forge } \\
\text { geese } \\
\text { germ } \\
\text { gig } \\
\text { gown } \\
\text { gush } \\
\text { herb } \\
\text { ledge } \\
\text { loaf } \\
\text { lurch } \\
\text { merge } \\
\text { mesh }\end{array}$ & & $\begin{array}{c}\text { morgue } \\
\text { moth } \\
\text { noose } \\
\text { notch } \\
\text { nudge } \\
\text { peg } \\
\text { pierce } \\
\text { shove } \\
\text { sieve } \\
\text { soothe } \\
\text { thatch } \\
\text { thief } \\
\text { thud } \\
\text { torch } \\
\text { turf } \\
\text { verse } \\
\text { vogue } \\
\text { void } \\
\text { web } \\
\text { wharf } \\
\text { yarn } \\
\text { zip } \\
\text { zoom }\end{array}$ \\
\hline \multicolumn{2}{|r|}{$S D$} & & $M$ & $S D$ \\
\hline \multicolumn{3}{|c|}{ Neighbourhood size } & 11.47 & 3.05 \\
\hline \multicolumn{3}{|c|}{ Word frequency } & 68.28 & 46.42 \\
\hline \multicolumn{5}{|c|}{ Neighbourhood Frequency } \\
\hline
\end{tabular}


Appendix B

Stimuli Used in Experiment 11

\begin{tabular}{llcc}
\hline \multicolumn{2}{c}{ Large neighbourhood } & \multicolumn{2}{c}{ Small neighbourhood } \\
High WF & Low WF & High WF & Low WF \\
\hline boat & bean & charge & chive \\
book & bike & death & fudge \\
face & cart & faith & garb \\
game & cheat & food & geese \\
lead & fad & guess & gong \\
heart & fin & house & hedge \\
hope & gash & jazz & keg \\
job & hop & judge & mesh \\
kill & lard & leg & moth \\
moon & mole & love & poise \\
park & rhyme & north & pouch \\
rain & rib & safe & sour \\
road & sage & sharp & surf \\
seat & shale & song & torch \\
tone & weed & south & vase \\
wife & whim & voice & verb
\end{tabular}

\begin{tabular}{|c|c|c|c|c|c|c|c|}
\hline$M$ & $S D$ & $M$ & $S D$ & $M$ & $S D$ & $M$ & $S D$ \\
\hline \multicolumn{8}{|c|}{ Neighbourhood size } \\
\hline 29.5 & 8.3 & 28.1 & 6.9 & 9.8 & 3.1 & 7.9 & 2.4 \\
\hline \multicolumn{8}{|c|}{ Word frequency } \\
\hline 145.1 & 87.4 & 2.3 & 1.6 & 163.4 & 137.8 & 2.4 & 1.6 \\
\hline \multicolumn{8}{|c|}{ Neighbourhood Frequency } \\
\hline 65.1 & 5.1 & 64.0 & 4.9 & 66.5 & 6.1 & 63.9 & 9.0 \\
\hline
\end{tabular}


